Universidad deValladolid

PROGRAMA DE DOCTORADO EN FISICA

\author{
TESIS DOCTORAL
}

\title{
Armonización de Datos de Satélite Mediante Zonas Homogéneas
}

\author{
Presentada por Juan Fernando Rodrigo Herrero \\ para optar al grado de \\ Doctor por la Universidad de Valladolid
}

Dirigida por:

Dra. Julia Sanz Justo

Dr. Pablo Salvador González

Dr. Diego Gómez Aragón

Valladolid, 2020 


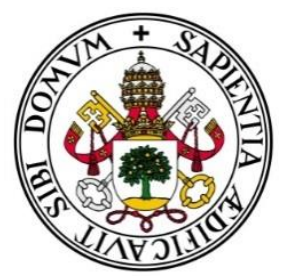

Satellite Data Harmonization Through Homogeneous Zones

Doctorate programme on Applied Physics "Earth observation, satellite calibration and validation"

\section{Juan Fernando Rodrigo Herrero}

PhD Advisor: Dr. José Luis Casanova (UVA, Spain)

PhD Director/s: Dra. Julia Sanz, Dr. Pablo Salvador and Dr. Diego Gómez (UVA, Spain) 
"Si queremos solucionar un problema que nunca antes habiamos resuelta. debemas dejar la puerta a la descanacida entreabierta.

R.P. Feynman 


\section{ESTRUCTURA DE LAS TESIS}

Esta tesis doctoral está estructurada en seis capítulos originales. En el primer capítulo, se realiza una sinopsis sobre la teledetección, las diferentes técnicas de calibración radiométrica, así como de los satélites y sensores utilizados en este trabajo. El capítulo dos corresponden a trabajos de investigación realizados para la presente tesis doctoral, que han sido publicados en Remote Sensing Journal (ISSN: 2072-4292) Special Issue "Cross-calibration and Interoperability of Remote Sensing Instruments" con un índice de impacto de 4.509 y posición 9 de 30 (Q2) en el año 2020 en la categoría de 'Remote Sensing' en la clasificación JCR. El capítulo tercero se está redactando para enviar a publicación en los próximos meses. El capítulo cuarto, también corresponde a trabajos de investigación publicados en Remote Sensing Journal (ISSN: 2072-4292) Special Issue "Cross-calibration and Interoperability of Remote Sensing Instruments" con un índice de impacto de 4.118 y posición 7 de 30 (Q1) en el año 2019 en la categoría de 'Remote Sensing' en la clasificación JCR. El capítulo quinto se corresponde con la discusión y conclusiones generales. Se finaliza con el listado e información relevante de los artículos de investigación publicados. Al final de cada capítulo, se incluye epígrafe de referencias bibliográficas con todas las citas que aparecen en el mismo.

Capítulo 1. Introducción

Capítulo 2. Armonización de datos de satélite a partir de una metodología global de SNOs y la utilización de áreas homogéneas.

Capítulo 3. Análisis radiométrico de la influencia de la RSRs en las bandas NIR de Sentinel 2 A/B mediante áreas homogéneas.

Capítulo 4. Análisis de la homogeneidad espacio-temporal del PICS Libya-4, para la localización de un área de calibración optimizado.

Capítulo 5. Discusión y conclusiones generales.

Capítulo 6. Lista de publicaciones. 


\section{ABSTRACT}

There is an increasing demand for remote sensing services and products in the society of today. In recent years the launch of new optical satellites has grown exponentially, enhancing Earth Observation programmes in terms of data accessibility and availability. Given the differences between sensors, products and their sources, an appropriate data harmonization is needed to improve their interoperability and facilitate their use.

Radiometric calibration is a key factor in data interoperability because it guarantees the scientific quality of the data and enables the comparison between sensors. The absolute radiometric calibration of a sensor is firstly undertaken at laboratory level before the satellite launch ("Pre-Launch") to determine the initial calibration parameters for an optimal performance. The launch stress and the exposition to the spatial working environment can lead to the degradation of the sensor response, modifying the initial reference values.

The absolute "Post-Launch" radiometric calibration allows us to adjust and compensate the sensor response, and therefore ensure the correct quality of the physical measurements. This type of calibration can be applied over different reference sources: on-board, In-Situ and Gold Standard. The on-board method uses a solar-diffuser panel or black body, the In-Situ method is applied over different locations on the Earth surface, and the Gold Standard applies Cross-Calibration between a perfect-calibrated sensor (constant data retrieval over the same location) and the un-calibrated sensor.

Most satellites do not have the necessary instruments to undertake the sensor calibration on-board for economic reasons (high cost, simpler satellite design, problems related to in-situ calibration in certain locations on the Earth surface). Thus, indirect calibration procedures (e.g. other sensors are used as reference) enable an accurate absolute radiometric calibration in a cost-effective and faster manner.

This doctoral thesis presents a methodology for satellite data harmonization based on the use of homogeneous areas. This method can be applied to two fundamental methodologies to undertake indirect radiometric calibration such as Pseudo-Invariant Calibration Sites (PICS) and Simultaneous Nadir Overpass (SNOs).

For satellite data Harmonization through the Cross-Calibration procedure based on the SNOs, an extension has been made of the original method developed by National Oceanic and Atmospheric Administration (NOAA/NESDIS). Multiple SNOs were identified and selected without orbital propagators from the L8-OLI, S2A-MSI and S2BMSI optical sensors. Subsequently, a wide range of reflectance values at Top-ofAtmosphere (TOA) were evaluated using the locations of homogeneous zones. In addition, the relation between $\mathrm{L} 8, \mathrm{~S} 2 \mathrm{~A}$ and S2B were investigated. The results showed high correlations between S2 A/B and $L 8$, with coefficients of determination $\left(R^{2}\right)$ greater than 0.980 for S2A and 0.984 for S2B. 
Given the weaker correlation between the near infrared (NIR) bands with different Relative Spectral Response (RSRs), the SNOs methodology was modified and adapted to further investigate the NIR bands of S2 satellites (bands 8 and $8 \mathrm{~A}$ ). The acquisition time for the NIR bands (in each S2 satellite) is 0 seconds. Thus, the atmospheric uncertainties, BRDF and scene light conditions associated to each acquisition are negligible (for each satellite) and therefore, the only differences across NIR bands are related to the effects of RSRs and the spectral surfaces investigated.

In the PICS methodology, the Optimized Ground Target (OGT) was determined by calculating the Coefficient of Variation (CV) along with the use of a quasi-Newton optimization algorithm combined with the Basin-Hopping global optimization technique. This work has identified an improved area of interest (AOI) within Libya-4. This AOI is more spatially homogeneous and temporally stable than the area proposed by The Committee on Earth Observation Satellites (CEOS), with differences between the CV of both zones in the order of $1 \%$ in the visible near-infrared (VNIR) bands of Landsat 8 (L8 - OLI) and Sentinel 2 (S2 - MSI).

The results shown in this doctoral thesis can improve the data Harmonization processes between high resolution sensors using an agile and global SNO methodology as well as, with a new AOI with more homogeneous and stable characteristics located in Libya-4. This thesis intends to improve the current techniques used to calibrate and validate (Cal/Val) optical satellite sensors, enhancing the harmonization and interoperability of Earth Observation data with scientific purposes.

Keywords: Remote Sensing; Harmonization; Interoperability: Radiometric calibration; Cross-Calibration; Homogeneous areas; Pseudo-Invariant Calibration Sites (PICS); Simultaneous Nadir Overpass (SNOs); Coefficient of Variation (CV); Relative Spectral Response (RSRs). 


\section{RESUMEN}

La sociedad actual, demanda de forma progresiva productos y servicios basados en datos de teledetección. En los últimos años el lanzamiento de nuevos satélites ópticos ha crecido de forma exponencial, aumentando la disponibilidad y acceso de datos de observación de la Tierra. Debido a las diferentes características entre sensores, los distintos tipos de productos y la diversidad de fuentes de los que provienen dichos datos, es necesario la armonización de los mismos para facilitar su uso eficiente e interoperabilidad.

La calibración radiométrica de los sensores es un factor clave en la interoperabilidad de los datos, permitiendo asegurar su calidad científica y la comparación de los mismos entre sensores. La calibración radiométrica absoluta de un sensor es inicialmente realizada en laboratorio antes del lanzamiento del satélite (Pre-Lanzamiento). Esto permite determinar los parámetros de calibración inicial para un óptimo funcionamiento del mismo. Las tensiones durante la fase de lanzamiento del satélite, junto con las condiciones de trabajo en el espacio, pueden provocar una degradación de la respuesta del sensor y por tanto una modificación de los valores de referencia inicial.

La calibración radiométrica absoluta (llevada a cabo durante la fase de PostLanzamiento) permite ajustar y compensar la respuesta del sensor, permitiendo asegurar la correcta calidad de las medidas físicas obtenidas por el mismo. Esta calibración se realiza sobre diferentes fuentes de referencia. Pueden realizarse mediciones a bordo del satélite (on board) mediante dispositivos localizados en el mismo, como un panel de difusor solar o un cuerpo negro. También es posible realizarlas In-situ sobre localizaciones de la superficie de la Tierra para una posterior comparación. Otra posibilidad, puede ser las medidas obtenidas por un sensor de referencia calibrado que adquiera datos de una zona de calibración de forma constante, para posteriormente "cruzarlos" (cross-calibration) con el sensor sin calibrar. Estos sensores de referencia son los denominados "Gold Standard".

Actualmente, la gran mayoría de satélites no poseen la instrumentación necesaria para realizar calibración on board por su elevado coste económico y por la simplicidad en el diseño del satélite lo que se une a la problemática de realizar medidas In-Situ en determinadas localizaciones de la Tierra. Por ello, la utilización de técnicas de calibración de manera indirecta tomando como referencia otros sensores permite una calibración radiométrica absoluta, de forma rápida, precisa y con un coste económico bajo.

En esta tesis doctoral, se presenta una metodología de armonización de datos de satélite a partir de zonas homogéneas, aplicable a las dos metodologías fundamentales para la calibración radiométrica de manera indirecta, como son Simultaneous Nadir Overpass (SNOs) y Pseudo-Invariant Calibration Sites (PICS). 
Para la armonización de datos mediante la cross-calibration basada en las SNOs, se ha realizado una ampliación de la metodología original desarrollada por National Oceanic and Atmospheric Administration (NOAA/NESDIS). Se identificaron y seleccionaron múltiples SNOs sin necesidad de propagadores orbitales para los sensores ópticos L8OLI, S2A-MSI y S2B-MSI. A continuación, mediante la localización de zonas espacialmente homogéneas, fue posible evaluar la relación entre las bandas L8 y las correspondientes de S2A y S2B para una amplia gama de valores de reflectancia Top-ofAtmosphere (TOA). Los resultados muestran altos coeficientes de determinación $\left(\mathrm{R}^{2}\right)$ para S2 A/B con respecto a L8, con valores superiores a 0.980 para S2A y 0.984 para S2B.

Debido a la menor correlación de las bandas NIR con diferente Relative Spectral Response (RSRs), se realizó una adaptación de la metologia de SNOs para el estudio de las bandas NIR 8-8A de S2 A/B. Al realizarse las adquisiciones de ambas bandas NIR de cada satélite $\mathrm{S} 2$ en un intervalo de tiempo nulo, las incertidumbres asociadas a efectos atmosféricos, de BRDF y de iluminación de la escena quedan eliminados, permitiendo observar únicamente los efectos debidos a las diferencias en las RSRs entre bandas y a las superfices espectrales utilizadas. Los resultados obtenidos muestran una gran correlación entre los pares de bandas NIR 8-8A a estudio de S2 A/B. A pesar de la diversidad de superficies utilizadas y las diferencias en su RSRs de ambas bandas NIR, el $\mathrm{R}^{2}$ muestra valores cercanos a 1 , con un valor de 0.998 tanto para S2A como para S2B.

Finalmente, para la metodología basada en PICS, con la aplicación del Coeficiente de Variación ( $C V$ junto con el uso de un algoritmo de optimización de quasi-Newton combinado con la técnica de optimización global Basin-Hopping, se ha identificado una zona de interés mejorada (AOI) dentro de Libya-4. Este nuevo AOI posee unas características más homogéneas y estables, tanto temporal como espacialmente, con respecto a la zona propuesta por The Committee on Earth Observation Satellites (CEOS). Las diferencias entre el $C V$ de ambas zonas son del orden del $1 \%$ en las bandas visibles e infrarrojo cercano (VNIR) de Landsat 8- OLI (L8-OLI) y Sentinel 2-MSI (S2-MSI).

Los resultados obtenidos en esta tesis doctoral permiten mejorar los procesos de armonización de datos de sensores de alta resolución con una nueva metodología de SNOs, ágil y global. Además, se propone un nuevo AOI localizado dentro de Libya-4 con características más homogéneas y estables. Por ello, se puede concluir que esta tesis doctoral progresa en el perfeccionamiento de las metodologías de Calibración y Validación (Cal/Val) de sensores de satélite. Especialmente importante, es el posible impacto de la misma, en la mejora de la armonización e interoperabilidad de los datos de observación de la tierra utilizados por la comunidad científica.

Palabras Clave: Teledetección; Armonización; Interoperabilidad; Calibración Radiométrica; Cross-Calibration; Zonas Homogéneas; Pseudo-Invariant Calibration Sites (PICS); Simultaneous Nadir Overpass (SNOs); Coeficiente de Variación (CV); Relative Spectral Response (RSRs). 


\section{TABLA DE CONTENIDOS}

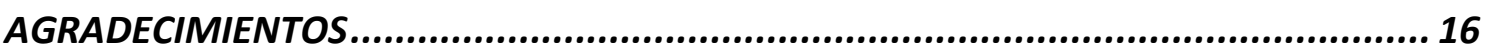

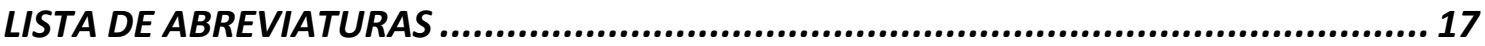

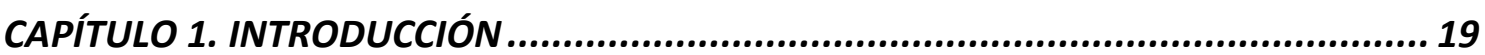

1.1. Teledetección ..............................................................................................19

1.2. Calibración y Validación de Sensores de Observación de la Tierra..........................19

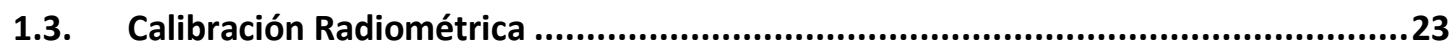

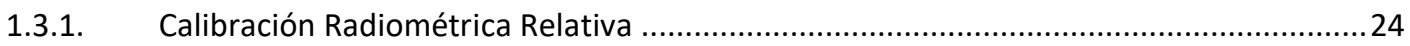

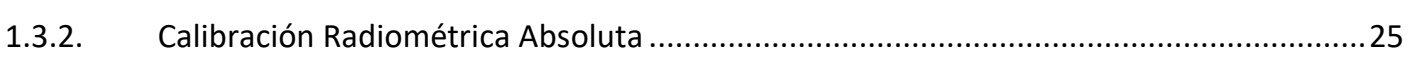

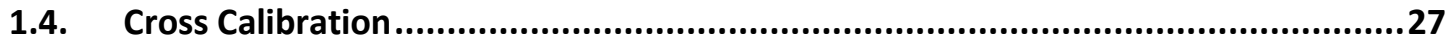

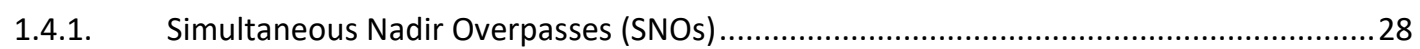

1.4.2. Pseudo-Invariant Calibration Sites (PICS) ................................................................. 29

1.5. Descripción General de los Sensores y Datos Utilizados.....................................30

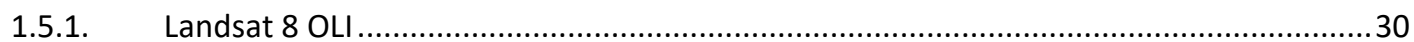

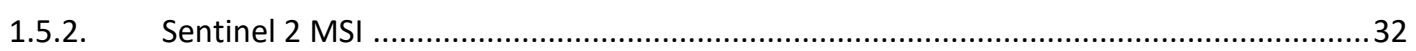

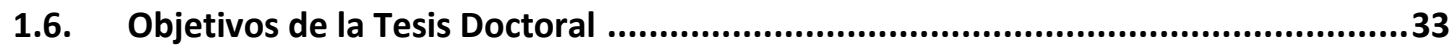

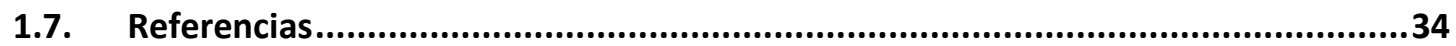

CAPÍTULO 2. ARMONIZACIÓN DE DATOS DE SATÉLITE A PARTIR DE UNA

METODOLOGÍA GLOBAL DE SNOSY LA UTILIZACIÓN DE ÁREAS HOMOGENEAS ....... 41

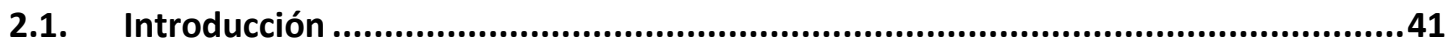

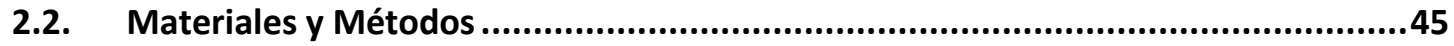

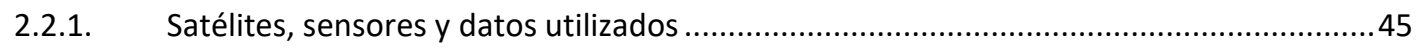

2.2.2. Determinación de la trayectoria terrestre de los satélites .............................................45

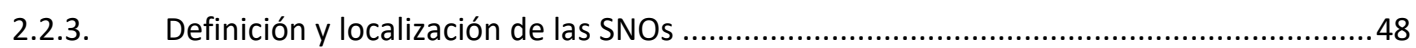

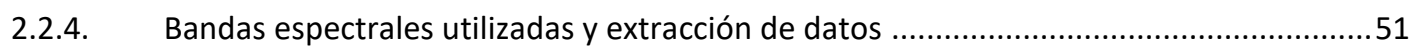

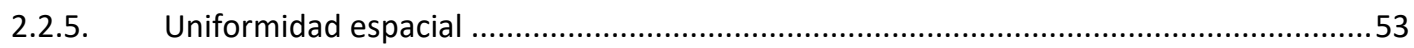

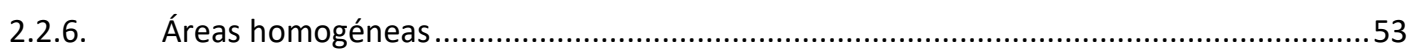

2.2.7. Comparativa entre sensores, análisis de datos y eliminación de outliers........................55

2.2.8. Análisis de las clases de superfices de suelo utilizadas................................................58

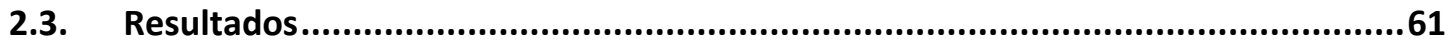

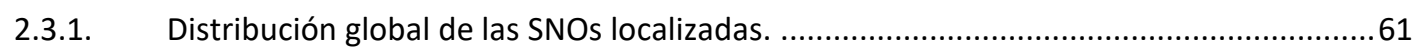

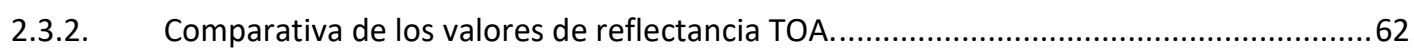

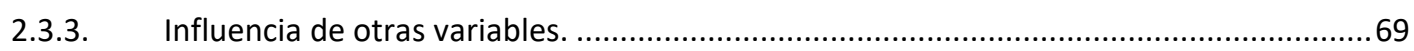

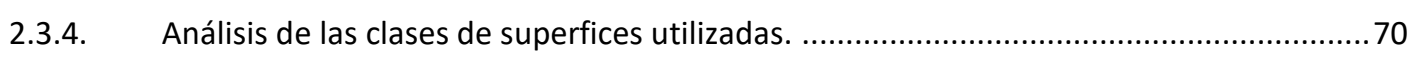

2.4. Discusión .............................................................................................................73

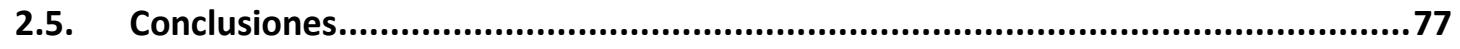

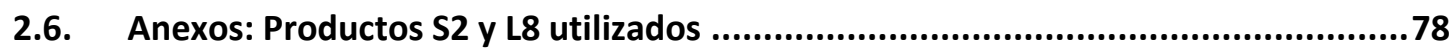


CAPÍTULO 3. ANÁLISIS RADIOMÉTRICO DE LA INFLUENCIA DE LA RSRS EN LAS BANDAS NIR DE SENTINEL 2 A/B MEDIANTE ÁREAS HOMOGÉNEAS.

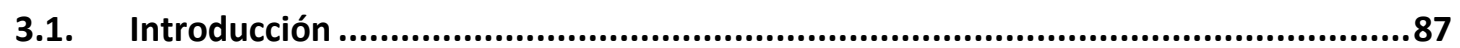

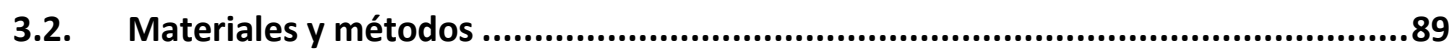

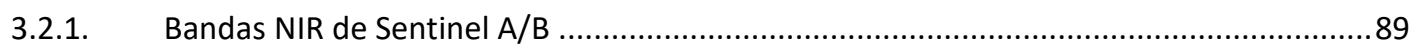

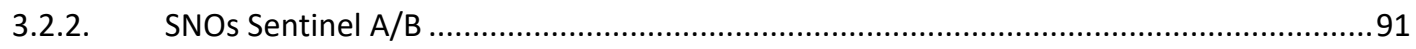

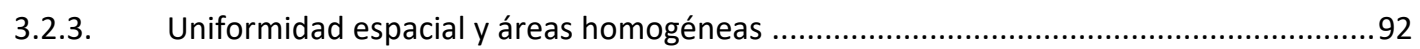

3.2.4. Comparativa entre sensores, análisis de datos y eliminación de outliers.......................93

3.2.5. Análisis de las clases de superficies de suelo utilizadas. ................................................94

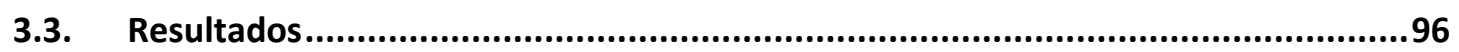

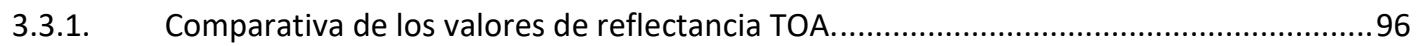

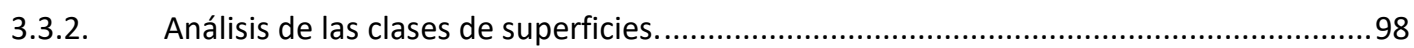

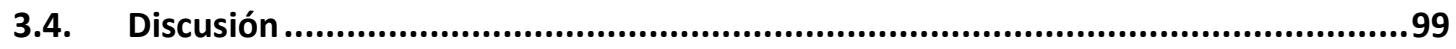

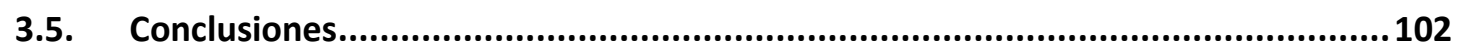

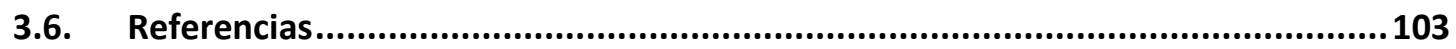

CAPÍTULO 4. ANÁLISIS DE LA HOMOGENEIDAD ESPACIO - TEMPORAL DEL PICS LIBYA-

4, PARA LA LOCALIZACIÓN DE UN ÁREA DE CALIBRACIÓN OPTIMIZADO ................ 107

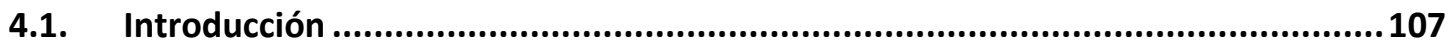

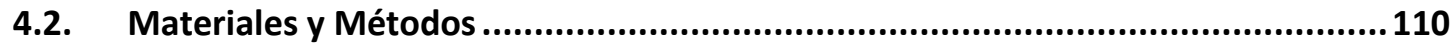

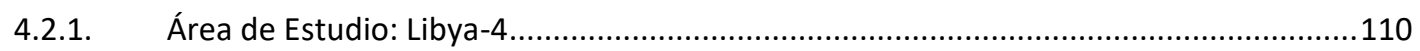

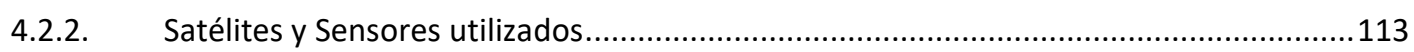

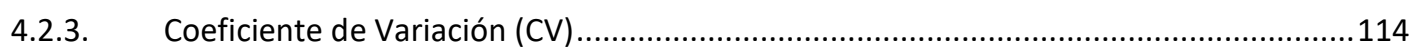

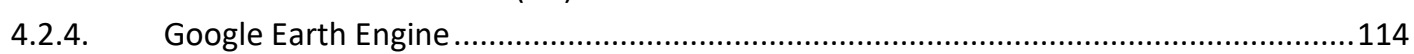

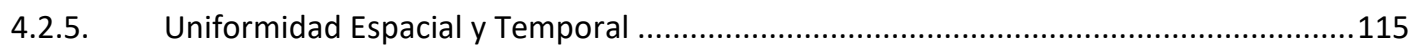

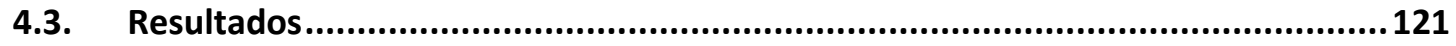

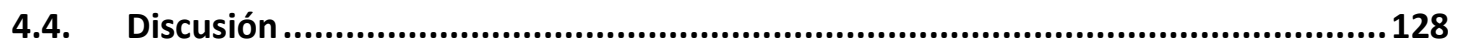

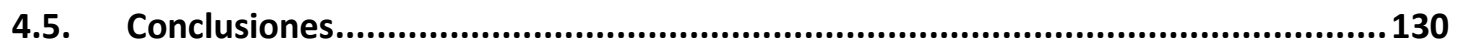

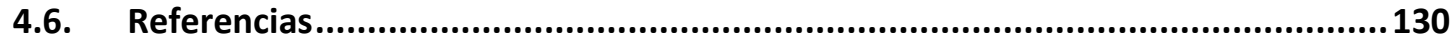

CAPÍTULO 5. DISCUSIÓN Y CONCLUSIONES GENERALES ................................... 137

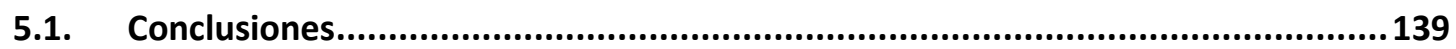

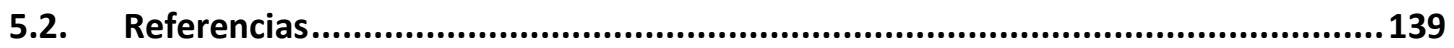

CAPÍTULO 6. LISTA DE PUBLICACIONES................................................... 143 


\section{AGRADECIMIENTOS}

En primer lugar, quisiera agradecer al Catedrático José Luis Casanova y a la Dr. Julia Sanz su apoyo, cercanía y soporte durante todos estos años. Ellos fueron los que me dieron la oportunidad de realizar este trabajo y de formar parte de la gran familia de la teledetección. Quisiera agradecer de manera muy especial a mis directores de tesis Dr. Pablo Salvador y Dr. Diego Gómez, por todo el tiempo, esfuerzo y cariño que me han dedicado.

Especial mención se merece Jorge Gil, una de esas personas que se cruzan en tu vida y que rebosan tanta sencillez como sabiduría.

Me faltan páginas en esta tesis, para agredecer a mis padres todo su esfuerzo, coraje y lucha que han realizado durante toda su vida por mi y mis hermanas, siempre sereis el espejo al que mirar. Muchas gracias a mis hermanas, que a pesar de la distancia que nos separa, siempre han estado ahí a pesar de todo.

Esta tesis está especialmente dedicada a las dos personas más importantes de mi vida, Naroa y Valeria. Cada palabra, ecuación e imagen de esta tesis fueron posible gracias a vosotras. No hay nada imposible para una familia donde se unen la Física y la Química.

Como no agradecer en esta tesis a mis amigos los físicos, no es fácil encontrar amistades con el mismo desorden mental que uno mismo. Especialmente dentro de ellos, quisiera agredecer de una forma muy cariñosa a Vidal las horas de charla y divagación, siempre nos quedará en nuestro recuerdo el verano del 2010.

Quiero hacer una dedicatoria especial a mi gran amigo Iñigo, compañero de montañas, volcanes, viajes y aventuras de la vida. Gracias por estar siempre ahí.

La familia muchas veces son los amigos que uno escoge, por ello, Matteo y Valeria, gracias por sentiros cerca en todo momento de mi vida. Sois mi gran familia italiana.

No puedo olvidarme en esta sección de mi suegra Encarna, gracias por tu afecto, cercanía y comprensión, espero continuar durante muchos años la tradición milenaria entre suegra y yerno de broncas, risas, junto con una montaña rusa de sentimientos.

Por último, quisiera finalizar esta sección de agradecimientos dando las gracias al resto de amigos y compañeros de mi vida personal como profesional, David, Juanma, Ruper, Marcos, Antonio, Cristina, Alejandro, Juanjo, Sonia... Pero sobre todo, me gustaría dar las gracias a todos los que estuvieron, están y estarán en mi vida, pero especialmente a los que cumplan los tres estados. 


\section{LISTA DE ABREVIATURAS}

AOI: Área de Interés

APICS: The South Dakota State University Absolute PICS

BH-GOT: Basin-Hopping Global Optimization Technique

BRDF: Bidirectional Reflectance Distribution Function

Cal/Val: Calibración y Validación

CEOS: International Committee on Earth Observation Satellites

CGLS-LC100: Copernicus Global Land Cover Layers Collection 2

CV: Coeficiente de Variación

$\mathrm{CV}_{\text {ST: }}$ CV Espacio-Temporal

DMU: Drag Make-Up

DN: Digital Number

EO: Earth observation

EPICS: Extended Pseudo Invariant Calibration Sites

EROS: Earth Resources Science and Observation Data Center

FOV: Field Of View

GEE: Google Earth Engine

IVOS: Infrared and Visible Optical Sensors

L8-OLI: Landsat 8 OLI

LM BFGS: Limited Memory Broyden Fletcher Goldfarb Shanno MGRS: Military Grid Reference System

MPC: The Copernicus Sentinel-2 Mission Performance Centre MSI: Multispectral Instrument

NDVI: Normalized Difference Vegetation Index

NOAA: National Oceanic and Atmospheric Administration

OGT: Optimized Ground Target

OLI: Operational Land Imager 
PICS: Pseudo-Invariant Calibration Sites

PRF: Point Response Function

RGBNIR: Red, Green, Blue, NIR

RSRs: Relative Spectral Response

S2 A/B-MSI: Sentinel 2 A/B-MSI

SBAF: Spectral Band Adjustment Factor

SNOs: Simultaneous Nadir Overpass

SWIR: Short-Wave Infrared

TDI: Time Delay Integration

TIRS: Thermal Infrared Sensor

TOA: Top of Atmosphere

UTM: Universal Transversal Mercator

VNIR: Visible and Near-Infrared

WGCV: Working Group on Calibration \& Validation

WRS-2: Second Worldwide Reference System -2 


\section{CAPÍTULO 1. INTRODUCCIÓN}

\subsection{Teledetección}

La teledetección es la ciencia, y en cierta medida el arte, de adquirir información sobre la superficie de la Tierra sin estar realmente en contacto con ella. Esto se hace detectando y registrando la energía reflejada, difundida o emitida, para a continuación procesar, analizar y finalmente aplicar toda esa información (CCRS, 2015). Estos datos son obtenidos por sensores a bordo de satétiles, aeronaves, tripuladas o no, incluso por sensores in situ, siendo los más demandados los provenientes de sensores de satélites.

Los satélites ópticos de Observación de la Tierra (EO), proporcionan información precisa y confiable sobre el estado de la atmósfera, océanos, costas, ríos, suelos, cultivos, bosques, ecosistemas, recursos naturales, hielo, nieve, infraestructuras, etc. Estos datos obtenidos mediante técnicas de teledetección, son utilizados de forma directa o indirecta por múltiples sectores económicos, además de primordiales para el correcto funcionamiento de muchas actividades cotidianas de la sociedad actual (Paganini et al., 2018). La precisión de los sensores de EO y la calidad de los productos generados a partir de ellos son fundamentales debido a su influencia y dependencia en la toma de decisiones con un gran impacto en la sociedad actual (Chander et al., 2013). Por lo tanto, la caracterización y calibración de estos instrumentos, especialmente la cuantificación de sus sesgos relativos y absolutos, son vitales para el monitoreo de los procesos de la superficie de la Tierra, que requieren de mediciones precisas (Chander et al., 2013; Barrientos et al., 2016). Las metodologías de Calibración y Validación (Cal/Val) se han convertido en un factor clave para abordar los sesgos entre sensores, la interoperabilidad y armonización entre instrumentos (Chander et al., 2013; Helder et al., 2018)

\subsection{Calibración y Validación de Sensores de Observación de la Tierra}

El Grupo de Trabajo sobre Calibración y Validación (WGCV) del International Committee on Earth Observation Satellites (CEOS) tiene como objetivo primordial garantizar la confianza en la precisión y calidad de los datos, así como en los productos de EO. También proporciona un foro de discusión para el intercambio de información sobre Cal/Val, incluyendo la coordinación de actividades cooperativas entre agencias y gobiernos. EI WGCV define la calibración en teledetección como el proceso de definir cuantitativamente la respuesta de un sistema, a las entradas de señal conocidas y controladas. Por otro lado, la validación es el proceso de evaluar por medios 
independientes la calidad de los productos de datos derivados de esos resultados del sistema (WGCV, 2020).

Las actividades de Cal/Val comienzan mucho antes de que el sensor se encuentre operativo a bordo del satélite, debiendo iniciarse desde la propia fase de diseño del mismo. Esta planificación temprana permite adaptar el diseño del instrumento a las actividades de Cal/Val que se van a llevar a cabo (Tansock et al., 2004). El objetivo de esta temprana organización es establecer el enfoque de calibración más eficiente para cumplir los requisitos de rendimiento establecidos para la misión, mientras se minimiza la incertidumbre, el costo y los posibles riesgos asociados al proceso de calibración (Tansock et al., 2015). Un ejemplo de planificación de actividades de calibración se muestra en la Figura 1.

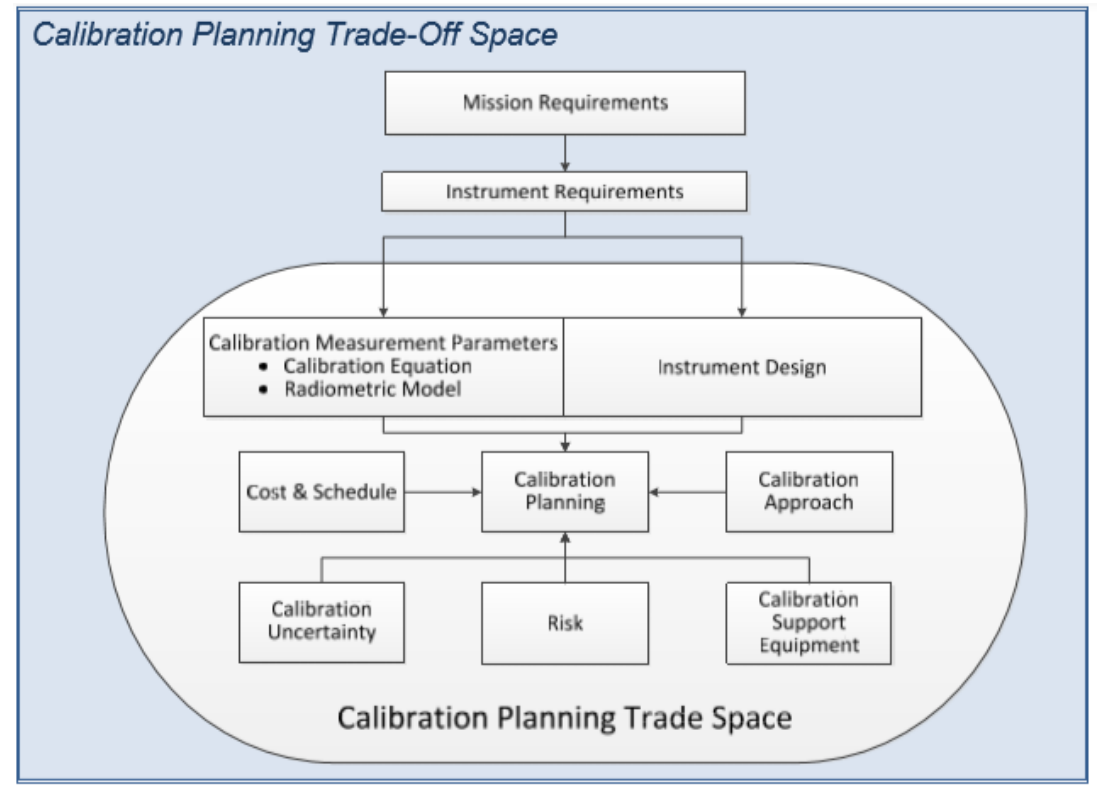

Figura 1. Planificación de las Actividades de Calibración. Fuente: (Tansock et al., 2015)

Las actividades de Cal/Val de los instrumentos de EO pueden dividirse en dos fases importantes, la fase de Pre-Lanzamiento y de Post-Lanzamiento. Las propiedades nominales del sensor en cada una de estas fases, experimentan cambios debido a las tensiones sufridas durante la fase de lanzamiento del satélite. También influyen las extremas condiciones de trabajo en el espacio, que pueden causar modificaciones en la calibración del instrumento con el paso del tiempo. Un ejemplo de ello, es la degradación de la óptica de la instrumentación como consecuencia de la exposición a la radiación ultravioleta (Tansock et al., 2015). Es importante afrontar esta problemática, ya que pequeños errores en la instrumentación tienen una considerable influencia en la calidad y precisión de los datos y productos generados por los mismos (Mlynczak et al., 2013).

Las actividades de Cal/Val durante la fase de Pre-Lanzamiento, posibilitan la realización de test $y$ ensayos en laboratorio, que facilitan mantener un control de las fuentes 
utilizadas, así como del entorno de pruebas. Esto permite caracterizar el instrumento, identificar, analizar y solucionar posibles anomalías antes de que el sensor sea lanzado. Las mediciones de Cal/Val realizadas durante la fase de Pre-Lanzamiento, permiten definir el funcionamiento nominal del sensor, consolidar el modelo radiométrico y establecer sus parámetros, así como las posibles incertidumbres asociadas. Especialmente importante en esta fase es evaluar y comprender el comportamiento del sensor, ya que es en esta fase cuando es posible ajustar o corregir esas anomalías de forma más fácil y sin un elevado impacto económico.

Una vez el satélite es puesto en órbita con toda su instrumentación, finaliza la etapa de Pre-Lanzamiento, dando comienzo la fase de Post-Lanzamiento. En esta fase, las condiciones de trabajo no van a ser simuladas, sino que corresponden a las condiciones reales de funcionamiento para las que fue diseñado el sensor. El objetivo principal durante este periodo, es mantener la calibración del sensor en un nivel óptimo durante toda su vida útil, con el fin de compensar la degradación natural del instrumento (Tansock et al., 2015). Durante el mismo, es necesario validar los resultados obtenidos mediante la comparación con otros sensores, con medidas in situ, u otro tipo de técnicas.

Algunas mediciones de Cal/Val tienen una mayor fiabilidad dependiendo en la fase en que se realicen. Durante la fase de Post-Lanzamiento, las mediciones en órbita pueden dar valores diferentes a las realizadas en laboratorio para algunos parámetros al realizarse en situaciones reales de funcionamiento. Un ejemplo es la medición de los parámetros Point Response Function (PRF) al usar la luna o las estrellas como fuentes puntuales ideales (Daniels et al., 2014), dark noise free y dark background/offset o los parámetros de apuntamiento del satélite. En la Figura 2 se muestra un ejemplo de los resultados de corrección de PRF obtenido a partir de observaciones lunares para el detector CERES.
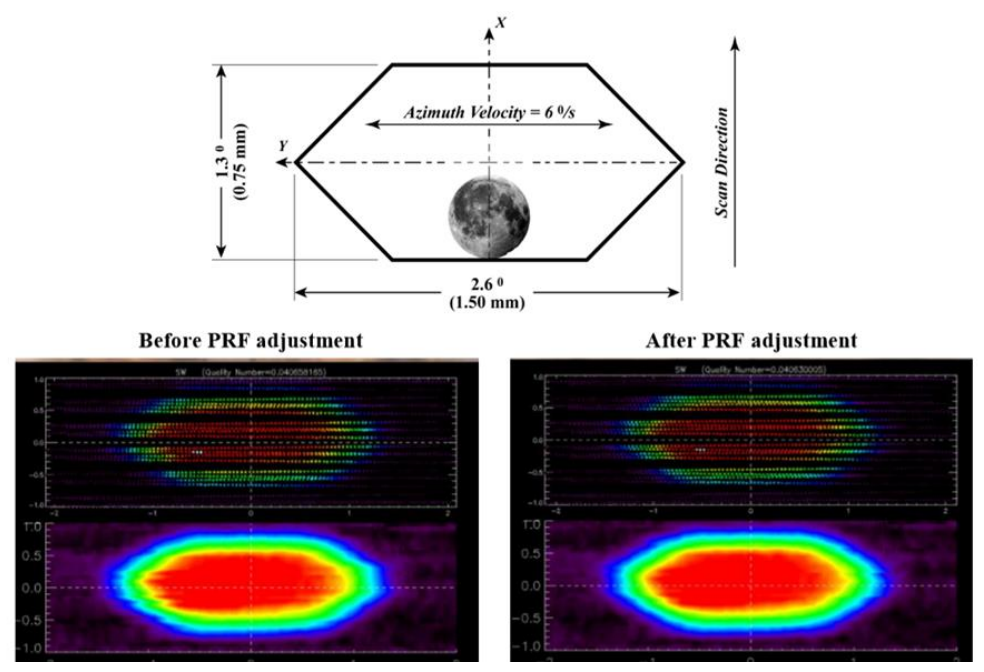

Figura 2. Corrección de PRF del canal de onda corta FM2. Fuente: Adaptado de (Daniels et al., 2014) 
En cambio, otros parámetros conviene calibrarlos en la fase Pre-launch, al no existir fuentes patrón que lo permitan o no ser lo suficientemente precisas. Por ejemplo, algunos de estos parámetros son la Relative Spectral Response (RSR), la eficiencia cuántica de los componentes, el comportamiento no lineal o la sensibilidad de polarización (Markham, 2019). En la Figura 3 y Figura 4 se muestran algunos de los test que se han realizado durante la fase de Pre-Lanzamiento al sensor OLI-2, que irá a bordo del satélite Landsat-9, cuya fecha prevista de lanzamiento está programada para diciembre de 2020.

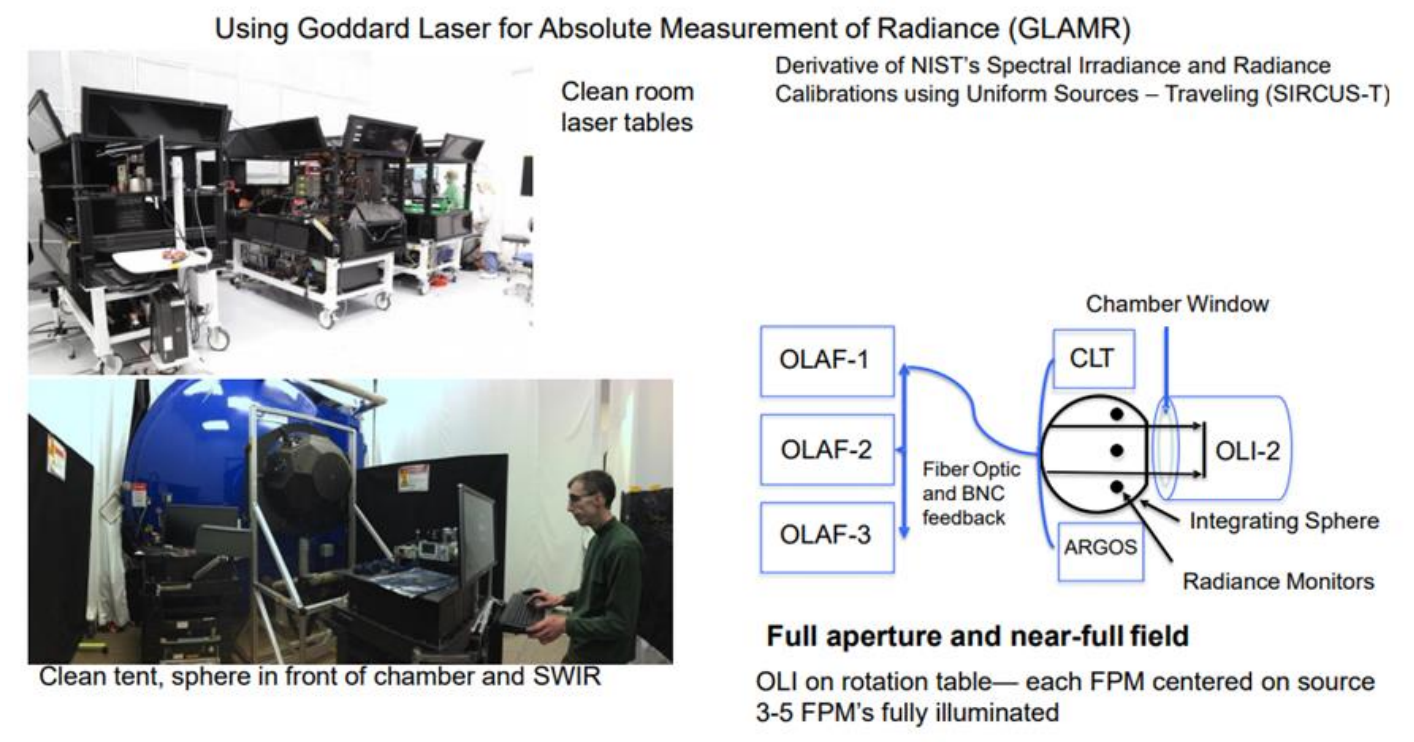

Figura 3. Configuración de prueba de la respuesta espectral del sensor OLI-2. Fuente: Adaptado de (Markham, 2019)

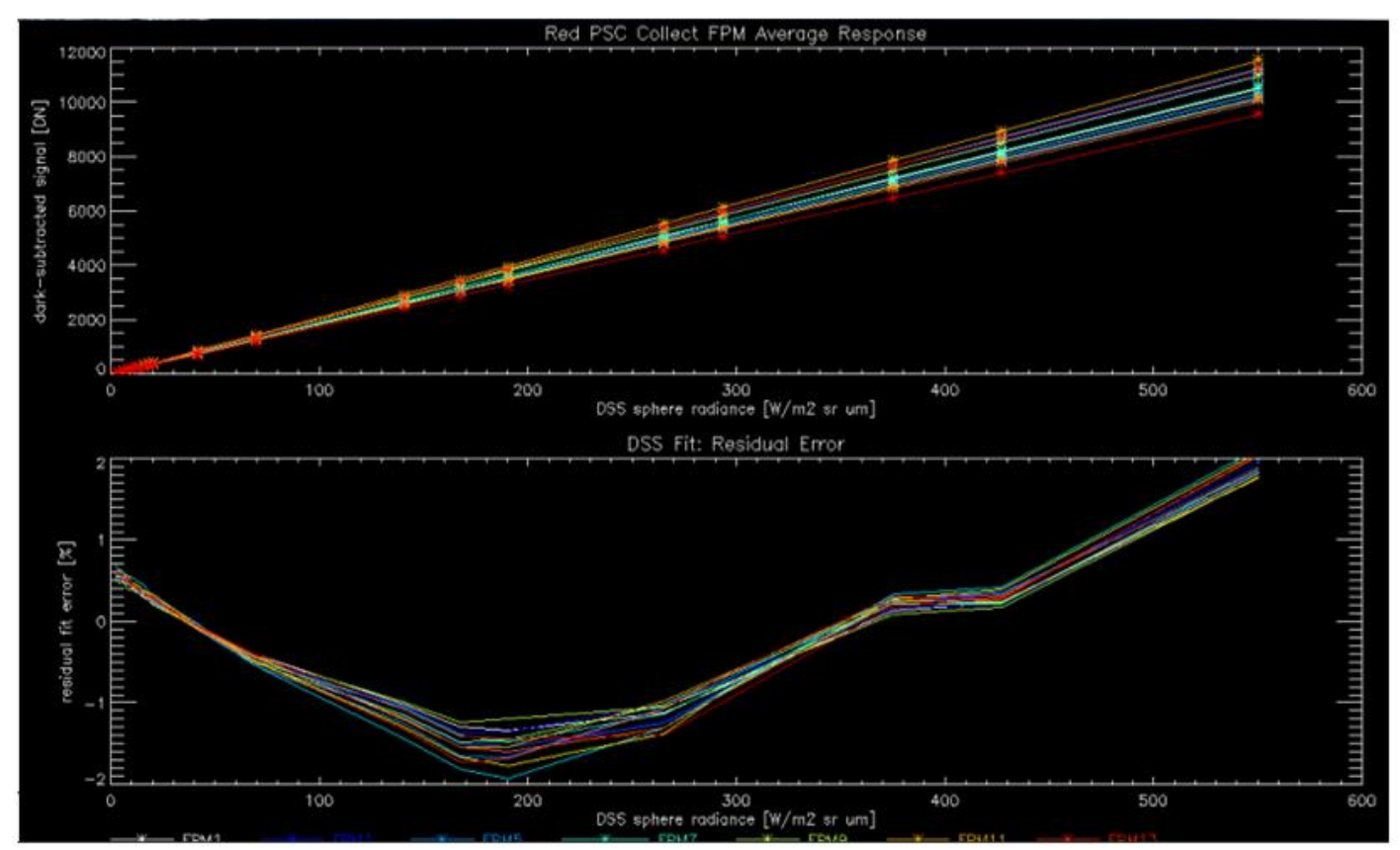

Figura 4. No linealidad de la Banda Roja del sensor OLI-2. Fuente: (Markham, 2019) 
De forma general, se puede dividir la calibración de los sensores de EO en cuatro grandes bloques: la parte geométrica, la espacial, las características espectrales del instrumento y la parte radiométrica del sensor (Markham, 2019). En esta tesis doctoral nos centramos en la armonización e interoperabilidad de datos de satélite usando técnicas de cross-calibration entre sensores y zonas homogéneas. Los resultados fueron comparados con diferentes metodologías para su validación.

\subsection{Calibración Radiométrica}

Los sensores a bordo de los satélites miden la cantidad de energía solar reflejada o difundida desde la superficie de la Tierra. Posteriormente, gracias a la electrónica del sensor, esa energía reflejada se convierte en números digitales (DNs) que permiten su conversión en imágenes. La calibración radiométrica se refiere a la capacidad de convertir en unidades físicas los DNs registrados por los sensores a bordo de un satélite. Esas unidades son la radiancia $(\mathrm{W} / \mathrm{m} 2 / \mathrm{sr} / \mu \mathrm{m})$ o la reflectancia Top of Atmosphere (TOA) (Figura 5). Las técnicas de calibración radiométrica intentan asegurar la fiabilidad de ese proceso de conversión (Helder et al., 2010).

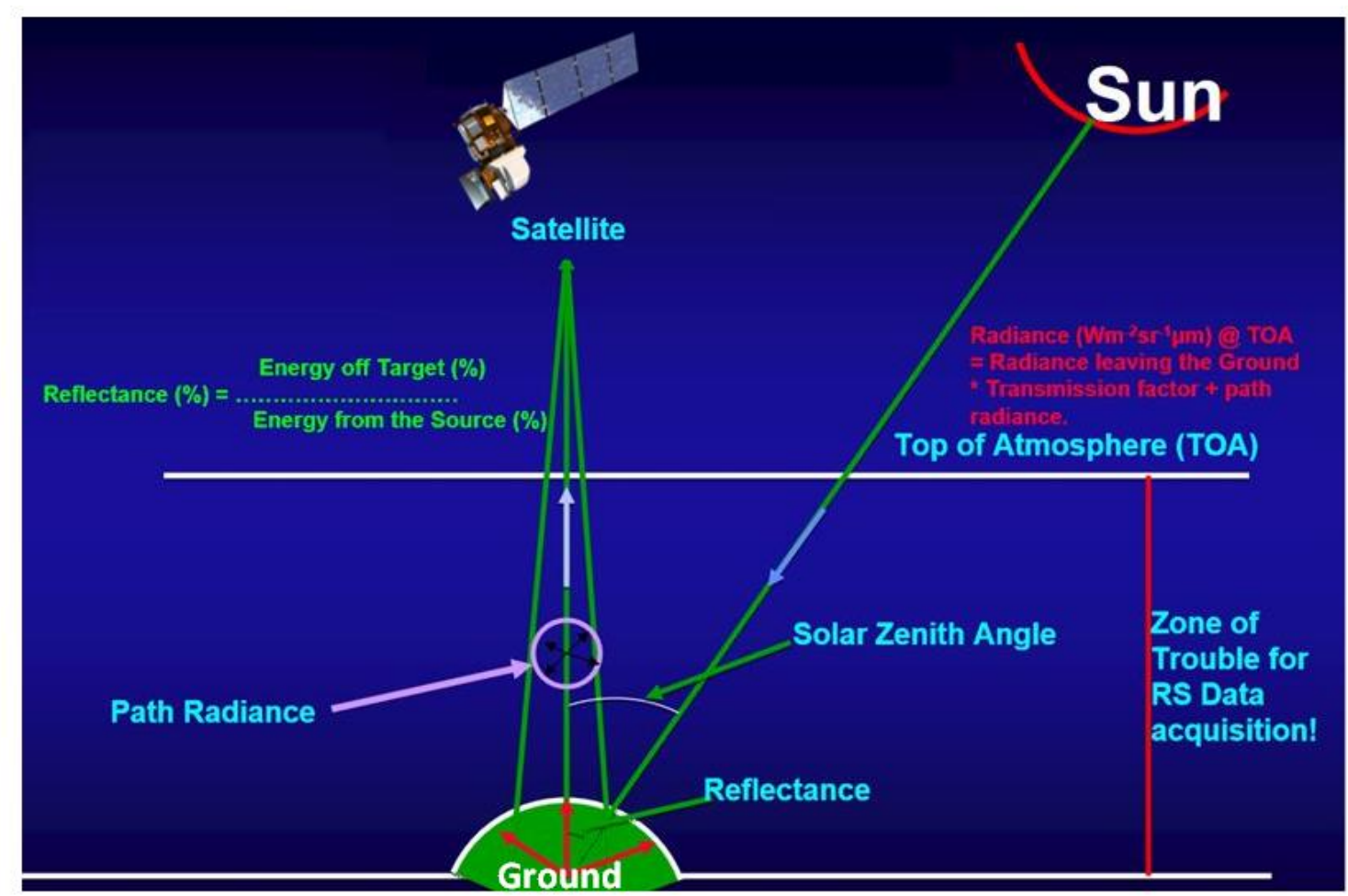

Figura 5. Ilustración de la radiancia y la reflectancia TOA. Fuente: Adaptado de (Thenkabail, 2009)

Pueden diferenciarse dos tipos fundamentales de calibración radiométrica en los sensores de satelite: la absoluta y la relativa (Thome et al., 1997; Tokola et al., 1999; Teillet et al., 2001; Vogelmann et al., 2001; Du et al., 2002). 


\subsubsection{Calibración Radiométrica Relativa}

Por la naturaleza del proceso de manufactura de los componentes electrónicos, es inevitable que cada uno de ellos presente una respuesta ligeramente distinta al resto ante el mismo nivel de señal. En un sensor push-broom, por ejemplo, esto se manifiesta con la presencia de líneas verticales en la imagen, que corresponden con la dirección de movimiento del satélite. Este fenómeno es conocido como "striping" (Figura 6).

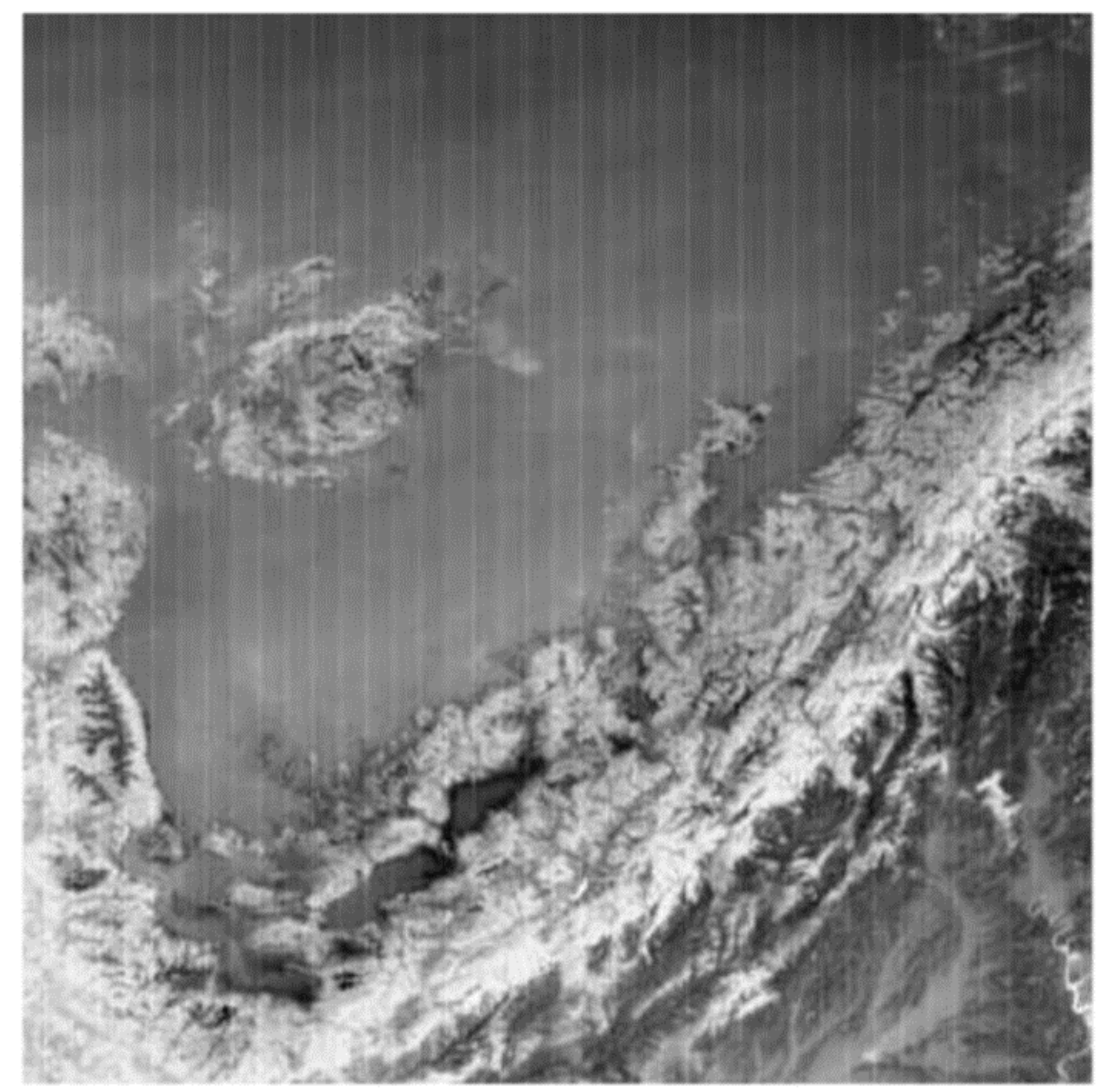

Figura 6. Imagen Terra-MODIS afectada por striping. Fuente: Chen et al., 2017.

Esta situación es causada por parámetros en los detectores no ajustados correctamente. Las imágenes generadas tendrán zonas más brillantes u oscuras que sus vecinas, creando lineas a lo largo del barrido del sensor. La calibración radiométrica relativa compensa las respuestas desiguales entre los detectores de un sensor de satélite. Para ello, se realiza el cálculo y aplicación de los parámetros que ecualizan la respuesta de los detectores cuando la radiación incidente es uniforme "flat field".

Debido a la degradación del sensor por el tiempo y las condiciones extremas de trabajo en el espacio, es recomendable realizar una campaña de calibración relativa periódica para calcular los nuevos coeficientes. En la fase de Post-Lanzamiento, es necesario adquirir imágenes sobre superficies muy uniformes con una alta reflectividad, especialmente en sensores sin dispositivos de calibración "on board". Dome-C es considerado un excelente target para la calibración radiométrica relativa de los sensores 
de EO (Six et al., 2004). Dome-C se encuentra situada en la parte oriental de la Antártida, con una elevación media aproximada de 3200 m, mínimo desnivel, una acumulación constante de nieve y una reflectividad muy uniforme y elevada (Walden et al., 2006; Pirazzini et al., 2004). En la Figura 7 se muestra un ejemplo de aplicación de los coeficientes de calibración relativa para el satélite de muy alta resolución Deimos-2, realizada sobre una imagen capturada sobre Dome-C (Gil et al., 2015).

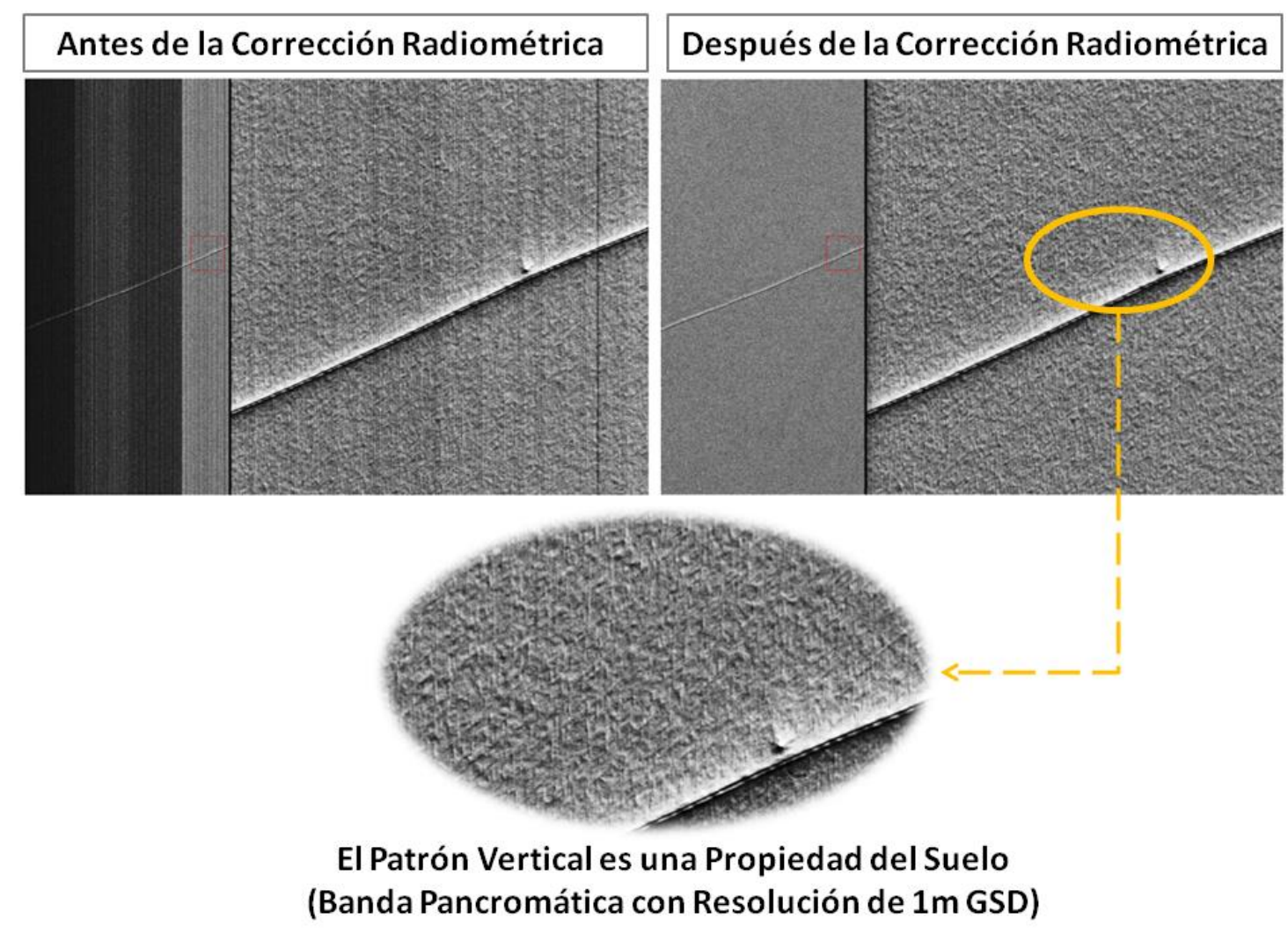

Figura 7. Calibración Relativa para la Banda Pancromática del Satélite Deimos-2.

Fuente: Adaptado de (Gil et al., 2015)

Como consecuencia del avance tecnológico, ha existido un aumento del número de detectores utilizados por los sensores de EO y de la complejidad de los mismos. Esto ha provocado una mayor precisión de las técnicas de calibración relativa, debido a su gran impacto en la calidad de las imágenes de satélite (Henderson \& Krause, 2004; Aramo et al., 2005; Pesta et al., 2015).

\subsubsection{Calibración Radiométrica Absoluta}

La calibración radiométrica absoluta es el proceso que convierte el voltaje o los recuentos digitalizados como DN y registrados por un sensor de satélite, en unidades físicas absolutas (radiancia, reflectancia, etc) (Mishra et al., 2014). Esta transformación es primordial para dar un significado físico a la información analógica o digital proveniente del sensor, para que sea posible la generación de datos y productos de calidad científica, así como la posterior comparación con otros instrumentos de otros 
satélites (Schowengerdt, 2007). De forma general, los coeficientes que permiten la conversión de los DNs de las imágenes a valores de radiancia o reflectancia son los Gains y Offset de cada una de las bandas. En sensores complejos, como por ejemplo con diferentes modos variables de time delay integration (TDI), pueden añadirse otros coeficientes de ajuste para la conversión de DN a unidades físicas. En la Ecuación 1 se muestra la fórmula general para la conversión de DN a radiancias (Liu, 2020), mientras que la Ecuación 2 es un ejemplo de conversión de diferentes sensores de la empresa Maxar, con valores de ajuste debido a la presencia de modos TDI en su instrumentación (Kuester, 2017). En la Figura 8 se muestra un ejemplo de coeficientes de calibración también para diferentes sensores de la empresa Maxar (Kuester, 2017).

$$
\begin{gathered}
L=G A I N * D N+\text { OFFSET (Eq. 1) } \\
L=G A I N * D N * \frac{\text { abscalfactor }}{\text { effectivebandwith }}+\text { OFFSET (Eq. 2) }
\end{gathered}
$$

Donde $L$ es la radiancia TOA en unidades de $W \mu \mathrm{m}^{-1} \mathrm{~m}^{-2} \mathrm{sr}^{-1}$, abscalfactor $\mathrm{y}$ effectivebandwith son los coeficientes específicos del modo TDI y del ancho de banda efectivo, DN es el valor de píxel y GAIN Y OFFSSET son los factores de

\begin{tabular}{|c|c|c|c|c|c|c|c|c|c|c|c|c|}
\hline \multirow{3}{*}{$\frac{\text { Cal Version }}{\text { BAND }}$} & \multicolumn{2}{|c|}{ WORLDVIEW-3 } & \multicolumn{2}{|c|}{ WORLDVIEW-2 } & \multirow{2}{*}{\multicolumn{2}{|c|}{$\begin{array}{c}\text { GEOEYE-1 } \\
2016 \text { v3.Int }{ }^{* *}\end{array}$}} & \multicolumn{2}{|c|}{ QUICKBIRD } & \multicolumn{2}{|c|}{ WORLDVIEW-1 } & \multirow{2}{*}{\multicolumn{2}{|c|}{\begin{tabular}{|l} 
IKONOS \\
$2014 \mathrm{v} 3$
\end{tabular}}} \\
\hline & \multicolumn{4}{|c|}{$2016 \mathrm{v} 0$. Int $^{*}$} & & & \multicolumn{4}{|c|}{ 2016v0.Int* } & & \\
\hline & GAIN & OFFSET & GAIN & OFFSET & GAIN & OFFSET & GAIN & OFFSET & GAIN & OFFSET & GAIN & OFFSET \\
\hline PAN & 0.950 & -3.629 & 0.942 & -2.704 & 0.970 & -1.926 & 0.870 & -1.491 & 1.016 & -1.824 & 0.907 & -4.461 \\
\hline COASTAL & 0.905 & -8.604 & 1.151 & -7.478 & & & & & & & & \\
\hline BLUE & 0.940 & -5.809 & 0.988 & -5.736 & 1.053 & -4.537 & 1.105 & -2.820 & & & 1.073 & -9.699 \\
\hline GREEN & 0.938 & -4.996 & 0.936 & -3.546 & 0.994 & -4.175 & 1.071 & -3.338 & & & 0.990 & -7.937 \\
\hline YELLOW & 0.962 & -3.649 & 0.949 & -3.564 & & & & & & & & \\
\hline RED & 0.964 & -3.021 & 0.952 & -2.512 & 0.998 & -3.754 & 1.060 & -2.954 & & & 0.940 & -4.767 \\
\hline REDEDGE & 1.000 & -4.521 & 0.974 & -4.120 & & & & & & & & \\
\hline NIR1 & 0.961 & -5.522 & 0.961 & -3.300 & 0.994 & -3.870 & 1.020 & -4.722 & & & 1.043 & -8.869 \\
\hline NIR2 & 0.978 & -2.992 & 1.002 & -2.891 & & & & & & & & \\
\hline SWIR1 & 1.200 & -5.546 & & & & & & & & & & \\
\hline SWIR2 & 1.227 & -2.600 & & & & & & & & & & \\
\hline SWIR3 & 1.199 & -2.309 & & & & & & & & & & \\
\hline SWIR4 & 1.196 & -1.676 & & & & & & & & & & \\
\hline SWIR5 & 1.262 & -0.705 & & & & & & & & & & \\
\hline SWIR6 & 1.314 & -0.669 & & & & & & & & & & \\
\hline SWIR7 & 1.346 & -0.512 & & & & & & & & & & \\
\hline SWIR8 & 1.376 & -0.372 & & & & & & & & & & \\
\hline
\end{tabular}
ajuste de calibración radiométrica absoluta.

**GE01 update on 6/6/2017

Figura 8. Coeficientes de Calibración de diferentes satélites de Maxar. Fuente: (Kuester., 2017)

La calibración absoluta es necesario realizarla de una forma continuada en el tiempo para poder evaluar el rendimiento del sensor durante toda su vida útil, desde la fase de Pre-Lanzamiento hasta el final de las operaciones del mismo. Esto permitirá detectar y corregir la degradación e incidencias en el sensor durante la fase de Post-Lanzamiento, modificando los parámetros de referencia inicial (Kuester, 2017). Este proceso posibilita 
seguir proporcionando datos con la mayor precisión radiométrica de forma constante a la comunidad de usuarios de EO (Mishra et al., 2014).

La calibración radiométrica absoluta durante la fase de Post-Lanzamiento puede realizarse empleando diferentes técnicas: instrumentación on-board, cross-calibration, calibración vicaria, uso de fuentes estelares y lunares, etc (Mishra et al., 2014). Debido al elevado coste de la instrumentación para la realización de calibración on-board y a la problemática de acceso por cuestiones geopolíticas, técnicas y económicas para la calibración vicaria, las técnicas de calibración indirecta tomando a los considerados sensores "Gold Standard" como referencia (Houborg et al., 2019; Stensaas, 2015) son las metodologías más utilizadas para las actividades de calibración radiométrica absoluta. Este tipo de técnicas permiten la monitorización de forma constante de los coeficientes de calibración, de un modo rápido, preciso y con un coste económico bajo.

\subsection{Cross Calibration}

El principio de cross-calibration consiste en comparar la radiación o reflectancia medida por el sensor a calibrar con la obtenida por otro sensor considerado de referencia o "Gold Standard" (Lachérade et al., 2013). La cross-calibration se realiza utilizando los datos de dos sensores adquiridos sobre el mismo target. La cross-calibration puede ser basada en unidades de radiancia o reflectancia. Para ambos y de forma general, se utiliza una recta de calibración para la obtención de los coeficientes de Gain y Offset como se muestra en las Ecuaciones 3 y 4 . En la Figura 9 se muestra de forma genérica el flujo de trabajo general para la cross-calibration entre sensores.

$$
\begin{aligned}
& L_{C A L}=\operatorname{Gain}_{L} * L_{R E F}+O f f \operatorname{set}_{L} \text { (Eq. 3) } \\
& \rho_{C A L}=\operatorname{Gain}_{\rho} * \rho_{R E F}+O f f \operatorname{set}_{\rho} \text { (Eq. 4) }
\end{aligned}
$$

Donde Gain $_{L}$ y Gain $_{\rho}$ es el coeficiente de ajuste Gain según se utilicen unidades de radiancia o reflectancia, $O f f$ set $_{L}$ y $O f f s e t_{\rho}$ es el coeficiente de ajuste offset según se utilicen valores de radiancia o reflectancia, $L_{R E F}$ y $\rho_{R E F}$ son la radiancia y reflectancia del sensor de referencia, mientras que $L_{C A L}$ y $\rho_{C A L}$ son la radiancia y reflectancia del sensor a calibrar. 


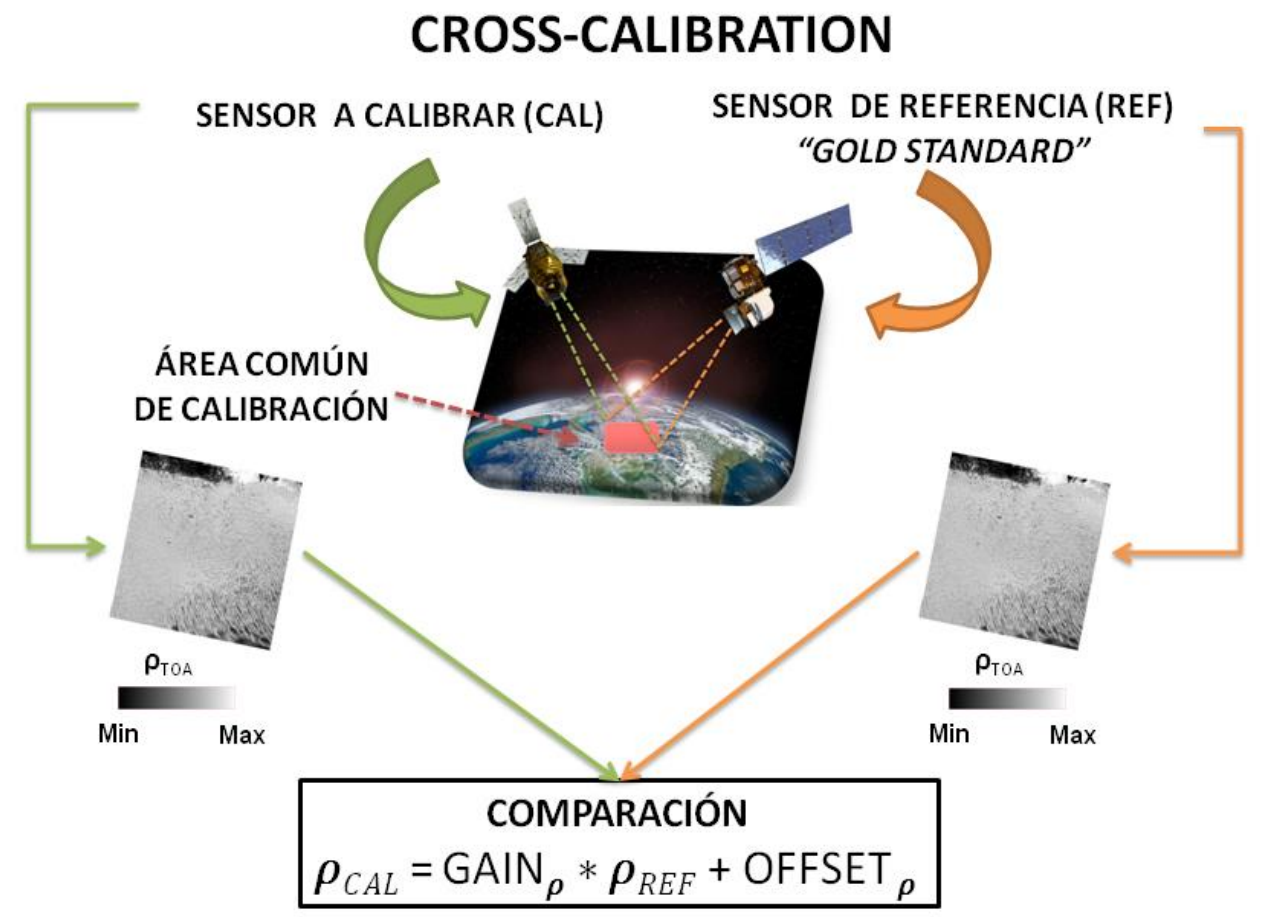

Figura 9. Flujo genérico de cross-calibration para dos sensores.

La cross-calibration se ha convertido en un factor clave en la consistencia y precisión de las series temporales de datos entre misiones (Lachérade et al., 2013), así como en la armonización e interoperabilidad de los mismos. Expecialmente en el actual contexto, con una gran variedad de sensores de EO que generan una alta y diversa cantidad de datos y productos.

Un claro ejemplo de este tipo de metodologías de cross-calibration son las Simultaneous Nadir Overpasses (SNOs) y los Pseudo-Invariant calibration sites (PICS).

\subsubsection{Simultaneous Nadir Overpasses (SNOs)}

En la cross-calibration, los sensores pueden realizar la adquisición de forma coincidente a nivel espacio-temporal con las mismas características geométricas de visualización. Un claro ejemplo de este tipo de casuística son las SNOs. Las SNOs consisten en la intercomparación directa y el posterior ajuste de las mediciones obtenidas por los sensores que vuelan a bordo de los satélites cuando estos sensores observan la misma superficie terrestre simultáneamente o en un intervalo de tiempo muy corto (Tansock et al., 2015; Uprety et al., 2013; Karlsson \& Johansson, 2014). El punto clave de la cross-calibration mediante SNOs es la eliminación y/o mitigación de factores de incertidumbre que puedan afectar a las medidas de los instrumentos, al realizarse las adquisiciones en nadir y de forma simultánea. La Bidirectional Reflectance Distribution Function (BRDF), la geometría de iluminación, la geometría de visión y la influencia atmosférica en las mediciones son prácticamente iguales para ambas observaciones (Tansock et al., 2015; Uprety et al., 2013; Karlsson \& Johansson, 2014), lo que permite el estudio directo del 
rendimiento radiométrico comparado de ambos sensores (Tansock et al., 2015; Karlsson \& Johansson, 2014).

\subsubsection{Pseudo-Invariant Calibration Sites (PICS)}

Normalmente en la cross-calibration, debido a las distintas características orbitales de los satélites (nodo ascendente o descendente, altura, frecuencia temporal, hora de paso por el ecuador, etc), las adquisiciones realizadas entre sensores sobre el mismo target, se realizan en condiciones diferentes tanto a nivel temporal como de condiciones geométricas (Lachérade et al., 2013). La metodología de cross-calibration mediante PICS es un claro ejemplo de lo mencionado anteriormente.

Los PICS son ubicaciones en la superficie de la Tierra con una gran estabilidad temporal y uniformidad espacial durante largos períodos de tiempo, así como con una extensión significativa (Chander, 2008). Debido a estas características especiales, los PICS han sido utilizados para la calibración absoluta Post-Lanzamiento, la monitorización de la estabilidad radiométrica, y especialmente para la cross-calibration entre sensores (Mishra et al., 2014; Angal et al., 2013). Los PICS se suelen ubicar en desiertos del Norte de África y Arabia Saudí con ausencia de vegetación y núcleos urbanos (Figura 10). Presentan una serie de características que les permiten ser grandes target de calibración, como son su alta reflectancia superficial, una excelente uniformidad espacio-temporal y gran superficie del suelo donde adquirir imágenes útiles en el proceso de calibración (Chander, 2008).

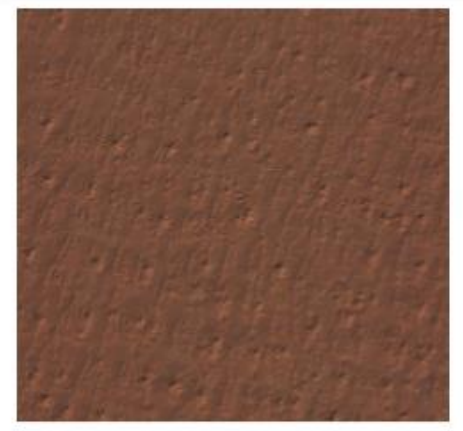

Algeria_3

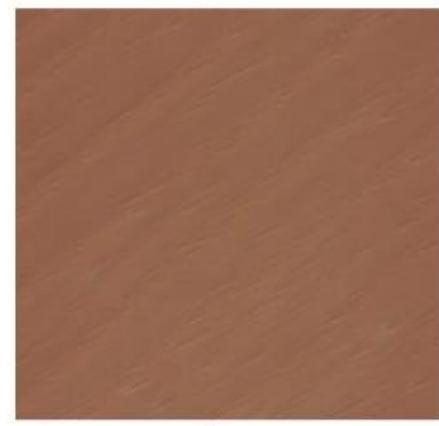

Libya_1

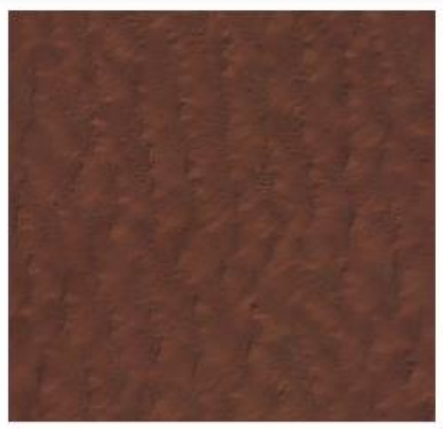

Algeria_5

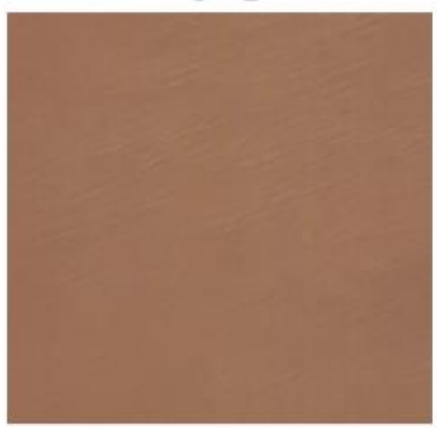

Mali_1

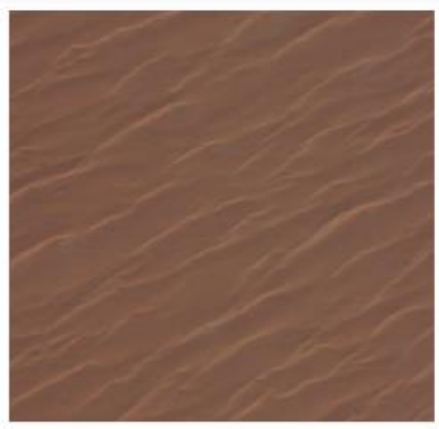

Arabia_1

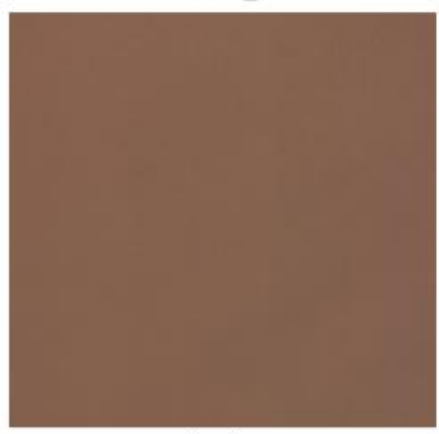

Nigeria_2

Figura 10.Imagen RGB de diferentes PICS.Fuente: (Lachérade et al., 2012) 
Uno de los factores influyentes en la cross-calibration entre sensores es la diferencia orbital de los satélites, que limitan las coincidencias espacio-temporales entre ellos. Además, la frecuencia temporal de adquisición sobre los PICS es baja para algunos sensores, como por ejemplo Landsat 8 OLI (L8-OLI), que es de una vez cada 16 días (Storey et al., 2014). Esta situación provoca que las adquisiciones de cada satélite se hayan realizado en condiciones diferentes de iluminación, de observación de los instrumentos y superficie dentro de los PICS. Esta situación tiene una influencia en los valores de reflectancia o radiancia medidos entre sensores debido a los efectos de la $B R D F$, haciendo necesario realizar una modelización de la BRDF para los valores de reflectancia o radiancia TOA obtenidos.

\subsection{Descripción General de los Sensores y Datos Utilizados}

En esta tesis doctoral se han utilizado los sensores L8-OLI and S2 A/B-MSI debido a la excelente calidad de sus datos (Gascon et al., 2017; Roy, 2014). Esto permite la utilización de cualquiera de ellos como "Gold Standard" según las características de la metodología de calibración a implementar.

Con respecto a los datos utilizados, se ha hecho uso de valores de reflectancia TOA. Cabe recordar que la conversión general de valores de Radiancia TOA a Reflectancia TOA se realiza aplicando la Ecuación 5:

$$
\rho_{\lambda}=\frac{\pi * L_{\lambda} * d^{2}}{E S U N_{\lambda} * \cos \theta_{S}} \text { (Eq. 5) }
$$

Donde $\rho_{\lambda}$ es el valor de reflectancia TOA que no tiene unidades, $L_{\lambda}$ es la radiancia espectral TOA en unidades de $W \mu \mathrm{m}^{-1} \mathrm{~m}^{-2} \mathrm{sr}^{-1}, d$ es la distancia Tierra-Sol en unidades astronómicas, $E S U N_{\lambda}$ es la media de la iradiancia solar extraterrestre en unidades de $W \mu \mathrm{m}^{-1} \mathrm{~m}^{-2} \mathrm{y} \cos \theta_{s}$ es el coseno del ángulo cenital solar en grados.

Actualmente para las imágenes de L8 se proveen factores de ajuste en los metadatos que permiten la conversión directa de valores de DN a reflectancia TOA, mientras que las imágenes S2 se distribuyen ya con valores de reflectancia TOA.

Para esta tesis doctoral se han utilizado datos de reflectancia TOA de L8-OLI y S2 A/BMSI. Para L8, se han utilizado los productos L1TP de la Collection 1 Tier 1 mientras que para S2, se han utilizado los productos L1C. Ambos tipos de productos se encuentran orto-rectificados a la proyección Universal Transversal Mercator (UTM/WGS84) (USGS, 2020a; ESA, 2020)

\subsubsection{Landsat 8 OLI}

L8 fue lanzado el 11 de febrero de 2013, desde la Base de la Fuerza Aérea de Vandenberg en una óbita polar heliosíncrona de baja excentricidad inclinada a 98.2 grados, con 16 días de ciclo de repetición, con un tiempo de cruce ecuatorial de 10:00 a.m. +/- 15 
minuto y una altitud de aproximada de $705 \mathrm{~km}$ (Storey et al., 2014). La Figura 11 muestra una recreación del satélite $L 8$.

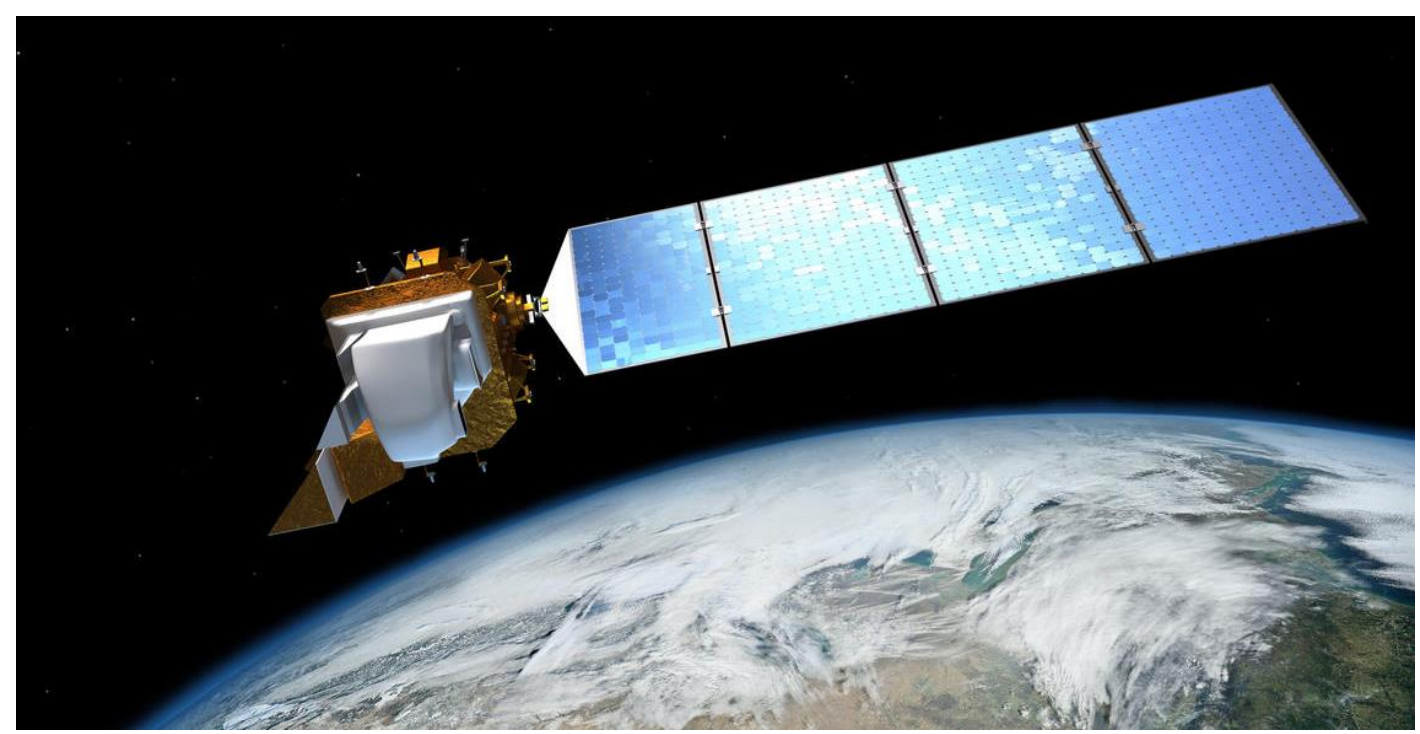

Figura 11. Ilustración de L8. Fuente: (USGS, 2020c)

A bordo de $\mathrm{L} 8$ se encuentran los instrumentos científicos Operational Land Imager (OLI) y el Thermal Infrared Sensor (TIRS) que tienen ambos un campo de visión de $15^{\circ}$ (FOV), proporcionando un swath de unos $185 \mathrm{~km}$. El sensor OLI tiene 9 bandas espectrales en el rango del visible e infrarrojo cercano (VNIR) e infrarrojo medio (SWIR) con una resolución espacial de $30 \mathrm{~m}$ para las bandas 1 a 7 y 9 , mientras que la resolución de la banda pancromática (banda 8) es de $15 \mathrm{~m}$ (Storey et al., 2014). En la Tabla 1 se muestran las características de las bandas de los sensores OLI e TIRS.

Tabla 1. Características de las Bandas de L8 para los sensores OLI y TIRS. Fuente: (USGS, 2020b)

\begin{tabular}{|c|c|c|c|}
\hline $\begin{array}{l}\text { Resolución } \\
\text { (metros) }\end{array}$ & $\begin{array}{l}\text { Número de } \\
\text { banda }\end{array}$ & $\begin{array}{l}\text { Banda } \\
\quad \#\end{array}$ & $\begin{array}{l}\text { Longitud de Onda } \\
\text { (micrómetros) }\end{array}$ \\
\hline 30 & 1 & Aerosol Costero & $0.43-0.45$ \\
\hline 30 & 2 & Blue & $0.45-0.51$ \\
\hline 30 & 3 & Green & $0.53-0.59$ \\
\hline 30 & 4 & Red & $0.64-0.67$ \\
\hline 30 & 5 & NIR & $0.85-0.88$ \\
\hline 30 & 6 & SWIR 1 & $1.57-1.65$ \\
\hline 30 & 7 & SWIR 2 & $2.11-2.29$ \\
\hline 15 & 8 & Pancromática & $0.50-0.68$ \\
\hline 30 & 9 & Cirrus & $1.36-1.38$ \\
\hline 100 & 10 & $\begin{array}{c}\text { Infrarrojo Térmico } \\
\text { (TIRS) } 1\end{array}$ & $10.6-11.19$ \\
\hline 100 & 11 & $\begin{array}{c}\text { Infrarrojo Térmico } \\
\text { (TIRS) } 2\end{array}$ & $11.50-12.51$ \\
\hline
\end{tabular}


1.5.2. Sentinel 2 MSI

La misión S2 forma parte del Programa Copernicus de la Comisión Europea para la vigilancia mundial de la superficie terrestre y las aguas costeras. Actualmente, el programa S2 consiste en una constelación de dos satélites gemelos, S2A y S2B, que fueron lanzados el 23 de junio de 2015 y el 7 de marzo de 2017, respectivamente, desde el puerto espacial de Europa cerca de Kourou en la Guayana Francesa. Están situados en una órbita polar heliosíncrona, compartiendo el mismo plano orbital con una diferencia de fase orbital de $180^{\circ}$ (Figura 12), a una altura aproximada de $786 \mathrm{~km}$ (Copernicus, 2015). Ambos satélites están equipados con la carga útil del MultiSpectral Instrument (MSI), que con un FOV de 20.6 $6^{\circ}$, proporciona un swath de unos $290 \mathrm{~km}$. El ciclo de repetición es de 10 días para cada satélite o 5 días para el uso combinado (Copernicus, 2015).

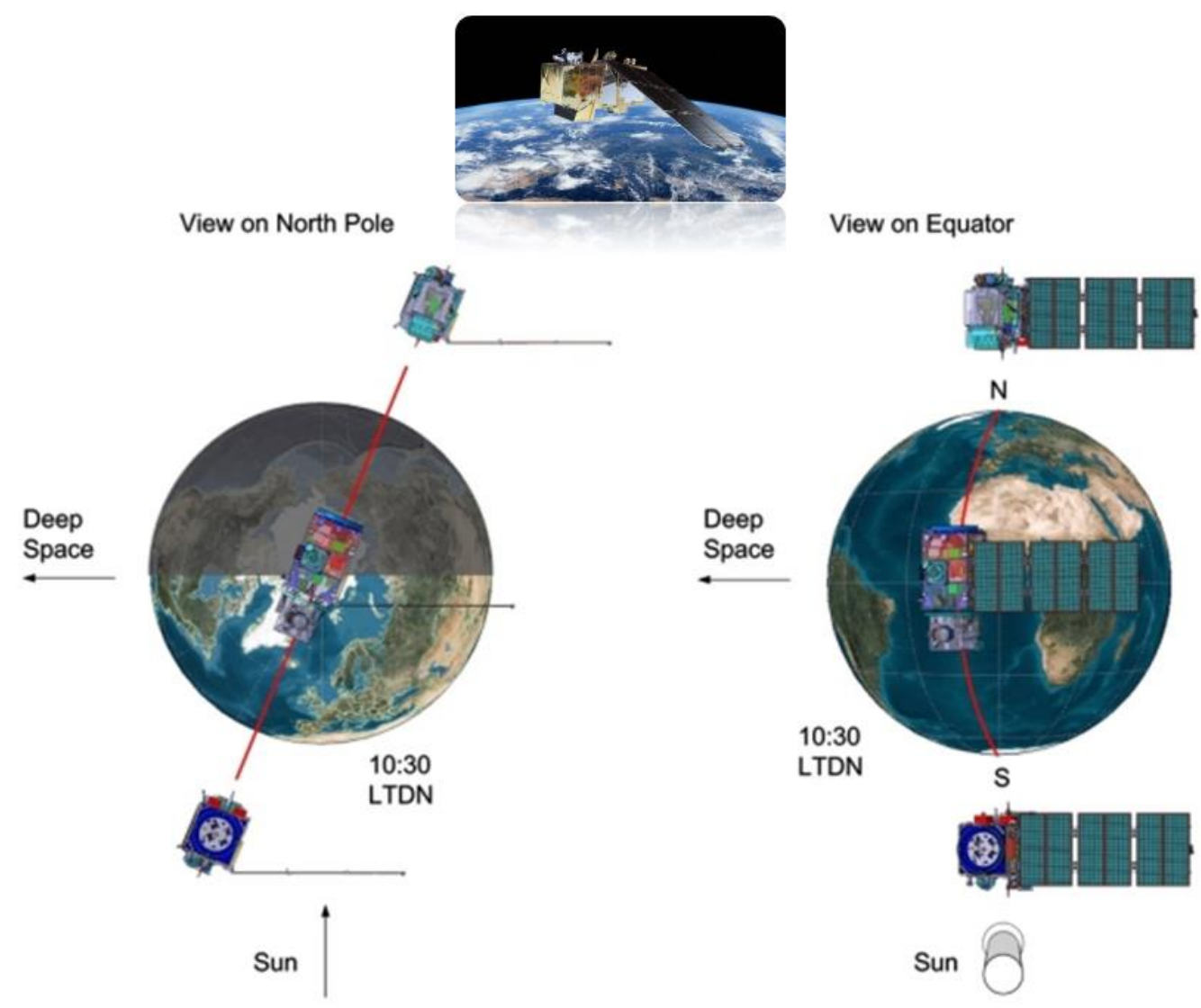

Figura 12. Configuración orbital de la misión S2. Fuente: Adaptada de (ESA, 2020)

EI MSI tiene 13 bandas espectrales en los dominios espectrales VNIR y SWIR con resoluciones espaciales de $10 \mathrm{~m}$ para las bandas 2, 3, 4 y 8, $20 \mathrm{~m}$ para las bandas 5, 6, 7, $8 \mathrm{~A}, 11,12$, y $60 \mathrm{~m}$ para las bandas 1, 9 y 10 (Copernicus, 2015). En la Tabla 2 se muestran las características de las bandas del sensor MSI para cada uno de los satélites S2. 
Tabla 2. Características de las Bandas del sensor MSI para cada satélite S2 A/B. Fuente: (ESA, 2020)

\begin{tabular}{|c|c|c|c|c|c|c|}
\hline \multirow[b]{2}{*}{$\begin{array}{l}\text { Resolución } \\
\text { espacial } \\
\text { (m) }\end{array}$} & \multirow[b]{2}{*}{$\begin{array}{l}\text { Número } \\
\text { de Banda }\end{array}$} & \multirow[b]{2}{*}{$\begin{array}{c}\text { Banda } \\
\#\end{array}$} & \multicolumn{2}{|c|}{ S2A } & \multicolumn{2}{|c|}{ S2B } \\
\hline & & & $\begin{array}{c}\lambda \text { Central } \\
(\mathrm{nm})\end{array}$ & $\begin{array}{c}\text { Ancho de } \\
\text { Banda } \\
\text { (nm) }\end{array}$ & $\begin{array}{c}\lambda \text { Central } \\
(\mathrm{nm})\end{array}$ & $\begin{array}{c}\text { Ancho de } \\
\text { Banda } \\
\text { (nm) }\end{array}$ \\
\hline \multirow{4}{*}{10} & 2 & Blue & 492.4 & 66 & 492.1 & 66 \\
\hline & 3 & Green & 559.8 & 36 & 559.0 & 36 \\
\hline & 4 & Red & 664.6 & 31 & 664.9 & 31 \\
\hline & 8 & NIR & 832.8 & 106 & 832.9 & 106 \\
\hline \multirow{6}{*}{20} & 5 & $\begin{array}{c}\text { Red Edge } \\
1\end{array}$ & 704.1 & 15 & 703.8 & 16 \\
\hline & 6 & $\begin{array}{c}\text { Red Edge } \\
2\end{array}$ & 740.5 & 15 & 739.1 & 15 \\
\hline & 7 & $\begin{array}{c}\text { Red Edge } \\
3\end{array}$ & 782.8 & 20 & 779.7 & 20 \\
\hline & $8 \mathrm{~A}$ & $\begin{array}{c}\text { NIR } \\
\text { Estrecha }\end{array}$ & 864.7 & 21 & 864.0 & 22 \\
\hline & 11 & SWIR & 1613.7 & 91 & 1610.4 & 94 \\
\hline & 12 & SWIR & 2202.4 & 175 & 2185.7 & 185 \\
\hline \multirow{3}{*}{60} & 1 & $\begin{array}{l}\text { Aerosol } \\
\text { Costero }\end{array}$ & 442.7 & 21 & 442.2 & 21 \\
\hline & 9 & $\begin{array}{c}\text { Vapor } \\
\text { de Agua }\end{array}$ & 945.1 & 20 & 943.2 & 21 \\
\hline & 10 & $\begin{array}{l}\text { SWIR- } \\
\text { Cirrus }\end{array}$ & 1373.5 & 31 & 1376.9 & 30 \\
\hline
\end{tabular}

\subsection{Objetivos de la Tesis Doctoral}

Esta tesis doctoral tiene como principal objetivo la mejora de las técnicas de armonización e interoperabilidad de los datos de EO. Dentro de las diferentes técnicas existentes, se ha apostado por la cross-calibration entre sensores. Para que los datos sean comparables e interoperables, los instrumentos deben de haberse sometido a un proceso de cross-calibration (Chander et al., 2013). Dentro de las técnicas de crosscalibration entre sensores destacan las metodologías de calibración radiométrica de manera indirecta, como son los SNOs y los PICS. En este trabajo, inicialmente se realizó una evaluación exhaustiva de estas metodologías para localizar posibles necesidades de mejora de cada una ellas.

Debido al fuerte crecimiento de satélites operativos sin calibración on board por el impacto económico en su coste operativo (Shrestha et al., 2019), así como a las diferentes características orbitales, técnicas y de diseño de los sensores a comparar, el número de oportunidades sobre los PICS es limitado e insuficiente para poder tener una inter-calibración ágil y lo más actualizada posible (Shrestha et al., 2019). Estos dos factores son fundamentales para la cross-calibration de sensores (NOAA, 2018; Helder et al., 2018; Shrestha et al., 2019). Para solventar esta problemática, en el capítulo dos y tres de esta tesis doctoral se presenta una metodología global de cross-calibration basada en SNOs a partir de la localización de zonas homogéneas que ha sido validada sobre una extensa serie de objetivos espectrales de la superficie terrestre. 
Para el caso de los PICS, dentro del subgrupo de trabajo Infrared and Visible Optical Sensors (IVOS) perteneciente a CEOS, se estableció en el 27 IVOS meeting del 2015, una nueva iniciativa llamada PICSCAR. El objetivo principal de la misma, es facilitar la coordinación científica y ayudar a establecer prioridades en la investigación sobre los PICS y su utilización en beneficio de la comunidad de usuarios de satélites ópticos (PICSCAR, 2020; ESA, 2017). Dentro de PICSCAR una de las problemáticas más importantes detectadas, es que no se está utilizando el mismo sitio de calibración dentro de cada PICS (ESA, 2017; Berthelot \& Henry, 2017). Reflejo de esta problemática fueron los resultados obtenido de una encuesta realizada por PICSCAR entre los usuarios que utilizan los PICS para actividades de Cal/Val (ESA, 2017; Berthelot \& Henry, 2017). Aunque los resultados muestran un consenso general en utilizar Libya-4 como el PICS de referencia debido a sus excelentes condiciones (homogeneidad espacio-temporal, estabilidad espectral y atmosférica), existe una falta de acuerdo en la utilización de un mismo Área de Interés (AOI). Muchos usuarios se refieren al AOI utilizado con el nombre de los PICS, por ejemplo, Libya-4, aunque no utilizan ni la misma localización, ni la misma extensión de superficie en la adquisición de datos (ESA, 2017; Berthelot \& Henry, 2017). La armonización y actualización de los vigentes targets de referencia de calibración es considerada una acción prioritaria (ESA, 2017; Berthelot \& Henry, 2017).

En el capítulo cuarto de esta tesis doctoral, se aborda la problemática de la falta de un target estándar de calibración en los PICS y en especial dentro de Libya-4 a partir de una novedosa metodología de estudio de la homogeneidad espacio-temporal. Con la implementación del Coeficiente de Variación (CV) junto con el uso de un algoritmo de optimización de Quasi-Newton combinado con la técnica de optimización global BasinHopping, se identificó un Optimized Ground Target (OGT) dentro de Libya-4, que permite seguir mejorando los procesos de armonización de datos entre diferentes sensores.

\subsection{Referencias}

Angal, A., Xiong, X., Choi, T., Chander, G., Mishra, N., \& Helder, D. L. (2013). Impact of Terra MODIS Collection 6 on long-term trending comparisons with Landsat 7 ETM+ reflective solar bands. Remote sensing letters, 4(9), 873-881.

Aramo, C., Brack, J., Caruso, R., D'Urso, D., Fazio, D., Fonte, Gemmeke, H., Kleifges, M., Knapik, R., Insolia, A. (2005). Optical relative calibration and stability monitoring for the Auger fluorescence detector. arXiv preprint astro-ph/0507577.

Barrientos, C., Mattar, C., Nakos, T., \& Perez, W. (2016). Radiometric Cross-calibration of the Chilean Satellite FASat-C Using RapidEye and EO-1 Hyperion Data and a Simultaneous Nadir Overpass Approach. Remote Sensing, 8(7), 612.

Barsi, J. A., Alhammoud, B., Czapla-Myers, J., Gascon, F., Haque, M. O., Kaewmanee, M., Leigh, L. \& Markham, B. L. (2018). Sentinel-2A MSI and Landsat-8 OLI radiometric 
cross comparison over desert sites. European Journal of Remote Sensing, 51(1), 822-837.

Berthelot, B., \& Henry, P. (2017). Radiometric Calibration Workshop for European Missions.https://earth.esa.int/documents/700255/3194632/B\%2B\%C2\%AEatri ce+Berthelot PICSCAR-PPT-022-

MAGv1.0 BBerthelot PHenry 20170830.pdf/9ddad0e8-532c-4f6c-9f19-

404c8280755c (Accedido el 24 Mayo de 2020)

CCRS, Canada Centre for Remote Sensing (2015). https://www.nrcan.gc.ca/maps-toolspublications/satellite-imagery-air-photos/remote-sensing-

tutorials/fundamentals-remote-sensing-introduction/9363 (Accedido el 15 de Mayo de 2020)

Chander, G., Hewison, T. J., Fox, N., Wu, X., Xiong, X., \& Blackwell, W. J. (2013). Overview of intercalibration of satellite instruments. IEEE Transactions on Geoscience and Remote Sensing, 51(3), 1056-1080.

Chander, G. (2008). Catalog of worldwide test sites for sensor calibration. In Proceedings of the JACIE Workshop 2008, 26-28 March 2008, Fairfax, Va.

Chen, Y., Huang, T. Z., Zhao, X. L., Deng, L. J., \& Huang, J. (2017). Stripe noise removal of remote sensing images by total variation regularization and group sparsity constraint. Remote Sensing, 9(6), 559.

Copernicus, (2015). Sentinel-2 User Handbook, Revision 2. https://sentinels.copernicus.eu/documents/247904/685211/Sentinel-

2 User Handbook (Accecido el 22 de Mayo de 2020)

Cosnefroy, H., Leroy, M., \& Briottet, X. (1996). Selection and characterization of Saharan and Arabian desert sites for the calibration of optical satellite sensors. Remote Sensing of Environment, 58(1), 101-114.

Daniels, J., Smith, G. L., Priestley, K. J., \& Thomas, S. (2014). Using lunar observations to validate pointing accuracy and geolocation, detector sensitivity stability and static point response of the CERES instruments. In Remote Sensing of Clouds and the Atmosphere XIX; and Optics in Atmospheric Propagation and Adaptive Systems XVII (Vol. 9242, p. 92420X). International Society for Optics and Photonics.

Du, Y., Teillet, P. M., \& Cihlar, J. (2002). Radiometric normalization of multitemporal high-resolution satellite images with quality control for land cover change detection. Remote sensing of Environment, 82(1), 123-134. 
ESA, (2017). Workshop on Radiometric Calibration for European Optical Missions, Frascati ESA/ESRIN, Italy. https://earth.esa.int/documents/700255/3194632/RadWS MoM Final.pdf/5fd20f6860b3-488a-a2bd-4732a3043a21 (Accedido el 24 de Mayo de 2020)

ESA, Sentinel 2. https://sentinel.esa.int/web/sentinel/missions/sentinel-2 (Accecido el 21 de Mayo de 2020)

Gascon, F., Bouzinac, C., Thépaut, O., Jung, M., Francesconi, B., Louis, J., Lonjou, V., Lafrance, B., Massera, S., Gaudel-Vacaresse, A., Languille, F., Alhammoud, B., Viallefont, F., Pflug, B., Bieniarz, J., Clerc, S., Pessiot, L., Trémas, T., Cadau, E., De Bonis, R., Isola, C., Martimort, P., Fernandez, V. (2017). Copernicus Sentinel-2A calibration and products validation status. Remote Sensing, 9(6), 584.

Gil, J., Romo, A., Moclan, C. \& Pirondini, F. (2015). Deimos-2 Post-launchPostLanzamiento radiometric calibration. 14th JACIE Workshop, Tampa, FL. https://spacedata.copernicus.eu/documents/12833/14537/DEIMOS-

2 RadiometricCalibration (Accedido el 18 de Mayo de 2020)

Helder, D. L., Basnet, B., \& Morstad, D. L. (2010). Optimized identification of worldwide radiometric pseudo-invariant calibration sites. Canadian Journal of Remote Sensing, 36(5), 527-539.

Helder, D., Markham, B., Morfitt, R., Storey, J., Barsi, J., Gascon, Clerc, S., LaFrance, B., Masek, J., Roy, D.P., Lewis, A., \& Pahlevan, N. (2018). Observations and Recommendations for the Calibration of Landsat $8 \mathrm{OLI}$ and Sentinel $2 \mathrm{MSI}$ for improved data interoperability. Remote Sensing, 10(9), 1340.

Henderson, B. G., \& Krause, K. S. (2004). Relative radiometric correction of QuickBird imagery using the side-slither technique on orbit. In Earth Observing Systems IX (Vol. 5542, pp. 426-436). International Society for Optics and Photonics.

Houborg, R., McCabe, M., \& Zuleta, I. (2019). Harmonization and inter-calibration of data streams from UAVs, CubeSats, Sentinel-2, and Landsat 8. AGUFM, 2019, B14A01.

Karlsson, K. G., \& Johansson, E. (2014). Multi-Sensor calibration studies of AVHRRheritage channel radiances using the simultaneous nadir observation approach. Remote Sensing, 6(3), 1845-1862.

Kuester, M. (2017). Absolute Radiometric Calibration: 2016v0. https://dg-cms-uploadsproduction.s3.amazonaws.com/uploads/document/file/209/ABSRADCAL FLEE T 2016v0 Rel20170606.pdf (Accedido el 19 de Mayo de 2020) 
Lacherade, S., Fougnie, B., Henry, P., \& Gamet, P. (2013). Cross calibration over desert sites: Description, methodology, and operational implementation. IEEE Transactions on Geoscience and Remote Sensing, 51(3), 1098-1113.

Lachérade, S., Fourest, S., Gamet, P., \& Lebègue, L. (2012). PLEIADES absolute calibration: inflight calibration sites and methodology. PAN, 1(B2), B3.

Liu, Y. K., Ma, L. L., Wang, N., Qian, Y. G., Zhao, Y. G., Qiu, S., Gao, C. X., Long, X. X., \& Li, C. R. (2020). On-orbit radiometric calibration of the optical sensors on-board SuperView-1 satellite using three independent methods. Optics Express, 28(8), 11085-11105.

Markham, B. (2019). Landsat 9 Pre-LaunchPre-Lanzamiento Instrument Characterization and Calibration. 18th JACIE Workshop, Reston, VA. https://calval.cr.usgs.gov/apps/sites/default/files/jacie/JACIE2019markham.pdf (Accedido el 17 de Mayo de 2020)

Mishra, N., Helder, D., Angal, A., Choi, J., \& Xiong, X. (2014). Absolute calibration of optical satellite sensors using Libya 4 pseudo invariant calibration site. Remote sensing, 6(2), 1327-1346.

Mlynczak, M. G., Hunt, L. A., Mast, J. C., Thomas Marshall, B., Russell III, J. M., Smith, A. K., Siskind, D., Yee, Y., Mertens, C., Martín, F., Thompson, R., Drob, D. \& Gordley, L. (2013). Atomic oxygen in the mesosphere and lower thermosphere derived from SABER: Algorithm theoretical basis and measurement uncertainty. Journal of Geophysical Research: Atmospheres, 118(11), 5724-5735.

NOAA, (2018). Advisory Committee on Commercial Remote Sensing (ACCRES). https://www.nesdis.noaa.gov/CRSRA/pdf/AACRES meeting 2018 Euroconsult. pdf (Accedido el 24 de Mayo de 2020).

Paganini, M., Petiteville, I., Ward, S., Dyke, G., Steventon, M., Harry, J., \& Kerblat, F. (2018). Satellite earth observations in support of the sustainable development goals. The CEOS Earth Observation Handbook.

Pesta, F., Bhatta, S., Helder, D., \& Mishra, N. (2015). Radiometric non-uniformity characterization and correction of landsat 8 oli using earth imagery-based techniques. Remote Sensing, 7(1), 430-446.

PICSCAR, CEOS WGCV IVOS initiative on the characterization of PICS. https://picscar.magellium.com/ (Accedido el 24 de Mayo de 2020)

Pirazzini, R. (2004). Surface albedo measurements over Antarctic sites in summer. Journal of Geophysical Research: Atmospheres, 109(D20). 
Roy, D. P., Wulder, M. A., Loveland, T. R., Woodcock, C. E., Allen, R. G., Anderson, M. C., Helder, D., Irons, J., Jhonson, D., Kennedy, T., Scambos, T. A., Schaaf, C., Schott, J., ... \& Zhu, Z. (2014). Landsat-8: Science and product vision for terrestrial global change research. Remote sensing of Environment, 145, 154-172.

Schowengerdt, R. A. (2007). Remote sensing, models and methods for image processing., Elservier Inc. New York, USA.

Shrestha, M., Leigh, L., \& Helder, D. (2019). Classification of North Africa for Use as an Extended Pseudo Invariant Calibration Sites (EPICS) for Radiometric Calibration and Stability Monitoring of Optical Satellite Sensors. Remote Sensing, 11(7), 875.

Six, D., Fily, M., Alvain, S., Henry, P., \& Benoist, J. P. (2004). Surface characterisation of the Dome Concordia area (Antarctica) as a potential satellite calibration site, using Spot 4/Vegetation instrument. Remote Sensing of Environment, 89(1), 8394.

Stensaas, G. (2015). USGS Report to GSICS EP-16. Global Space-based Inter-Calibration System Executive Panel, 16th Session, Boulder, CO, USA. http://www.wmo.int/pages/prog/sat/meetings/documents/GSICS-EP16 Doc 09 USGS-Report-2015 v3.pdf (Accedido el 19 de Mayo de 2020)

Storey, J., Choate, M., \& Lee, K. (2014). Landsat 8 Operational Land Imager on-orbit geometric calibration and performance. Remote sensing, 6(11), 11127-11152.

Storey, J., Choate, M., \& Lee, K. (2014). Landsat 8 Operational Land Imager on-orbit geometric calibration and performance. Remote sensing, 6(11), 11127-11152.

Tansock, J., Bancroft, D., Butler, J., Cao, C., Datla, R., Hanse, S., Helder, D., Kacker, R., Latvakoski, H., Mlynczak, M., Murdock, T., Peterson, J., Pollock, D., Russell, R., Scott, D., Seamons, J., Stone, T., Thurgood, A., Williams, R., Xiong, X., \& Yoon, H. (2015). Guidelines for Radiometric Calibration of Electro-optical Instruments for Remote Sensing (No. Handbook (NIST HB)-157.

Tansock, J., Thurgood, A., \& Larsen, M. (2004). System-level approach to characterization and radiometric calibration of a space-based electro-optical sensor. In Post-LaunchPost-Lanzamiento Calibration of Satellite Sensors (pp. 67102). CRC Press.

Teillet, P. M., Barker, J. L., Markham, B. L., Irish, R. R., Fedosejevs, G., \& Storey, J. C. (2001). Radiometric cross-calibration of the Landsat-7 ETM+ and Landsat-5 TM sensors based on tandem data sets. Remote sensing of Environment, 78(1-2), 3954. 
Thenkabail, P. (2009). Satellite Sensor Data Normalization Issues A User Perspective. Landsat Science Team Data Normalization Workshop, Boston, USA. http://www.pancroma.com/downloads/General\%20Landsat.pdf (Accedido el 18 de Mayo de 2020)

Thorne, K., Markharn, B., Barker, P. S., \& Biggar, S. J. P. E. (1997). Radiometric calibration of Landsat. Photogrammetric Engineering \& Remote Sensing, 63(7), 853-858.

Tokola, T., Löfman, S., \& Erkkilä, A. (1999). Relative calibration of multitemporal Landsat data for forest cover change detection. Remote sensing of environment, 68(1), 1 11.

Uprety, S., Cao, C., Xiong, X., Blonski, S., Wu, A., \& Shao, X. (2013). Radiometric intercomparison between Suomi-NPP VIIRS and Aqua MODIS reflective solar bands using simultaneous nadir overpass in the low latitudes. Journal of Atmospheric and Oceanic Technology, 30(12), 2720-2736.

USGS, Landsat 8 illustration above Earth. https://www.usgs.gov/media/images/landsat8-illustration-above-earth (Accedido el 22 de Mayo de 2020c)

USGS, Landsat Collection 1. https://www.usgs.gov/land-resources/nli/landsat/landsatcollection-1?qt-science support page related con=1\#qtscience support page related_con (Accedido el 21 de Mayo de 2020a)

USGS, What are the band designations for the Landsat satellites?. https://www.usgs.gov/land-resources/nli/landsat/landsat-collection-1?qtscience support page related con=1\#qt-science support page related con (Accedido el 22 de Mayo de 2020b)

Vogelmann, J. E., Helder, D., Morfitt, R., Choate, M. J., Merchant, J. W., \& Bulley, H. (2001). Effects of Landsat 5 Thematic Mapper and Landsat 7 Enhanced Thematic Mapper Plus radiometric and geometric calibrations and corrections on landscape characterization. Remote sensing of environment, 78(1-2), 55-70.

Walden, V. P., Roth, W. L., Stone, R. S., \& Halter, B. (2006). Radiometric validation of the atmospheric infrared sounder over the Antarctic plateau. Journal of Geophysical Research: Atmospheres, 111(D9).

WGCV, CEOS. http://ceos.org/ourwork/workinggroups/wgcv/ (Accedido el 15 de Mayo de 2020) 


\section{CAPÍTULO 2. ARMONIZACIÓN DE DATOS DE SATÉLITE A PARTIR DE UNA METODOLOGÍA GLOBAL DE SNO $S$ LA UTILIZACIÓN DE ÁREAS HOMOGENEAS}

\subsection{Introducción}

La vida media de las misiones de satélites se ha visto reducida para minimizar los riesgos asociados a los cambios en la demanda del mercado de EO (Öhrwall \& Isaksson, 2018). Las misiones con un rango de permanencia operativa menor con respecto a las misiones tradicionales con tiempos de vida útil por encima de los 15 años, son capaces de afrontar de una manera más eficiente y competitiva los desafíos y exigencias de los usuarios de datos de EO. Esta flexibilidad permite bajar los costes operativos de las misiones con componentes de menor duración y menor precio (Öhrwall \& Isaksson, 2018). De forma generalizada, la ausencia de dispositivos de calibración on-board en este tipo de misiones es un factor clave para su equilibrio operacional a nivel financiero (Hasan et al., 2019). Además, las diferentes configuraciones y características de los sensores que vuelan a bordo de los satélites generan una gran diversidad de datos de EO. Estos dos elementos han provocado que las metodologías de calibración Post-Lanzamiento indirectos sean consideradas un factor clave para mantener la calidad científica e interoperabilidad de los datos (Helder et al., 2018).

La Cross-calibration de los sensores se ha convertido en un factor fundamental para que los datos sean armonizables e interoperables (Chander et al., 2013), especialmente en el contexto actual de datos de EO (NOAA, 2018). Este proceso debe realizarse independientemente de la presencia de dispositivos de calibración on-board en los satélites a estudio. Durante décadas, se ha estado utilizando los PICS como referencia de calibración, especialmente en los satélites sin calibración a bordo para la crosscalibration (Helder et al., 2013; Angal et al., 2014; Mishra et al., 2014; Barsi et al., 2018). Las metodologías basadas en PICS necesitan largas series temporales de datos, modelización BRDF, cierta estabilidad atmosférica, además de estar restingidos a un determinado rango los valores de reflectancia o radiancia obtenidos. Asimismo, existe el problema de la elevada latencia en la disponibilidad de datos sobre los PICS.

Las SNOs se han convertido en una metodología de cross-calibration óptima, rápida, flexible en su aplicación y con un bajo coste. Las SNOs eliminan la necesidad de utilización de largas series temporales, de modelización de la BRDF y especialmente minimizan los efectos de las incertidumbres creadas por la atmósfera. Esta metodología de cross-calibration Post-Lanzamiento, utiliza el mismo fundamento empleado en la 
cross-calibration de sensores en fase Pre-Lanzamiento, que consiste en la adquisición y posterior comparación de los resultados sobre un mismo target de calibración (normalmente una esfera integradora o cuerpo negro) en las mismas condiciones de visualización y adquisición (Tansock et al., 2015).

La metodología de SNOs fue desarrollada por NOAA-NESDIS hace más de una década para la calibración entre satélites meteorológicos de órbita polar (Cao \& Heidinger 2002; Heidinger et al., 2002; Cao et al., 2004; Cao et al., 2005; Zou et al., 2006). Las SNOs minimizan las incertidumbres derivadas de las adquisiciones realizadas sobre la superficie de la Tierra por parte de los sensores de satélite, al observar el mismo target con apenas unos segundos de diferencia con idénticas condiciones de iluminación, geometría y atmósfera. Cao et al. (2004) desarrollaron una metodología de predicción de SNOs aplicada a los satélites NOAA-16, NOAA-17, Terra y Aqua a partir de un propagador de órbitas SGP4. Establecieron como criterios para la determinación del área de SNO el cruce de órbitas entre satélites, una diferencia entre adquisiciones menor de 30 segundos para evitar la presencia de nubes, así como un límite máximo de distancia al nadir de $420 \mathrm{~km}$ (Figura 13). Las SNOs de esta metodología quedaban restringidas a las regiones polares de la Tierra, normalmente entre el rango de latitudes $70^{\circ}-80^{\circ} \mathrm{N}$ y $70^{\circ}-80^{\circ} \mathrm{S}$ (Cao et al., 2004) como se muestra en la Figura 14. Estas restricciones limitan bastante el proceso de cross-calibration tanto a nivel espacial, temporal y del rango de valores de reflectancia o radiancia utilizados en la comparativa.

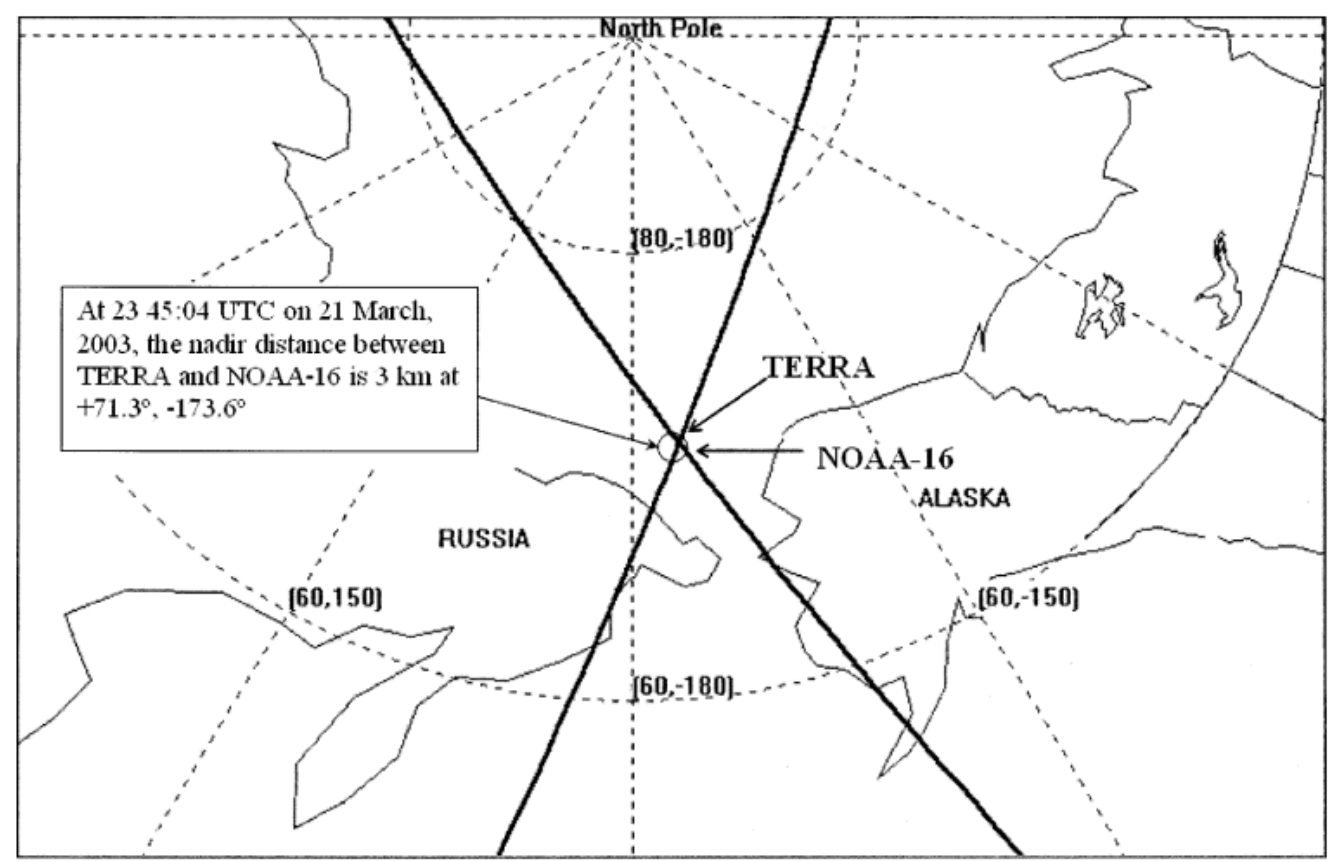

Figura 13. Ejemplo de SNO entre Terra y NOAA-16 con 1 segundo de diferencia. Fuente: Cao et al., (2004) 

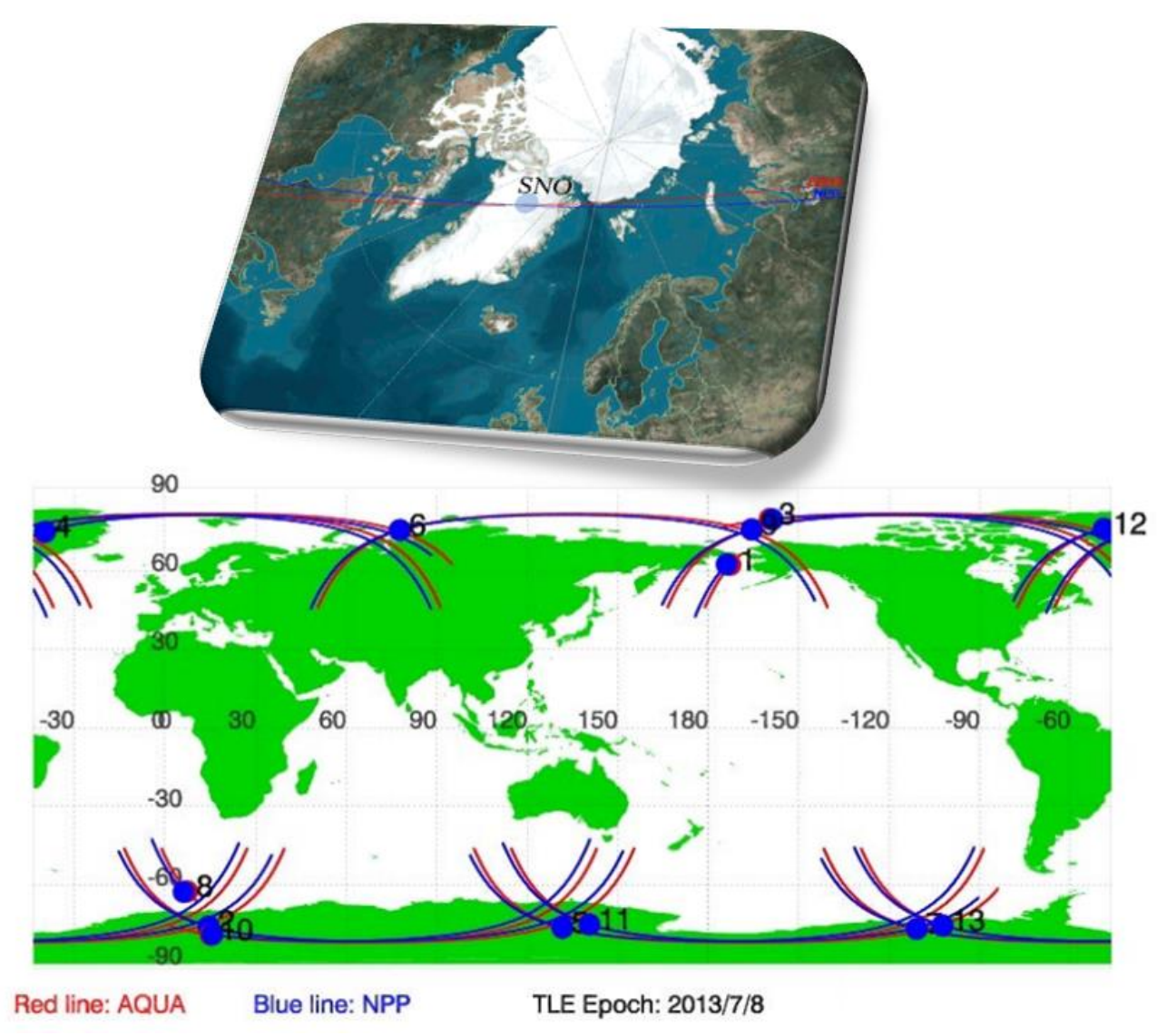

Figura 14. Predicción de SNOs en regiones polares de la Tierra. Fuente: Adaptado de Uprety et al., (2013)

Debido a los beneficios de esta metodología de cross-calibration, posteriormente se popularizó su uso entre la mayoría de satélites de EO, especialmente los que no tenían calibración on-board, convirtiéndose en una metodología esencial para la interoperabilidad de los datos (Chander et al., 2013).

Las restricciones utilizadas inicialmente para el cálculo de SNOs han sufrido modificaciones, especialmente las relacionadas con las condiciones orbitales y el tiempo de adquisición, siendo este último factor el más utilizado por la comunidad científica para la determinación de las SNOs (Tansock et al., 2015). Actualmente, se están utilizando los PICS para la cross-calibration entre sensores mediante la metodología de las SNOs sin necesidad de que las orbitas entre satélites se crucen o que la diferencia entre adquisiciones simultaneas se produzcan en un periodo muy corto de tiempo (Lacherade et al., 2013; Helder et al., 2012; Chander et al., 2013; Barsi et al., 2108). Los PICS poseen una gran superficie homogénea utilizable para tareas de Cal/Val y una atmósfera bastante estable, por tanto, pueden relajarse las restricciones de tiempo entre adquisiciones para la cross-calibration entre sensores mediante SNOs. Estas observaciones entre sensores pueden realizarse incluso con días de diferencia (Tansock et al., 2015). En la Figura 15 se muestra un ejemplo de las SNOs utilizadas por Barsi et 
al. (2018) sobre Libya-4 para S2 y L8 sin la restricción de cruce de órbitas y un mayor rango de tiempo en las adquisiciones.

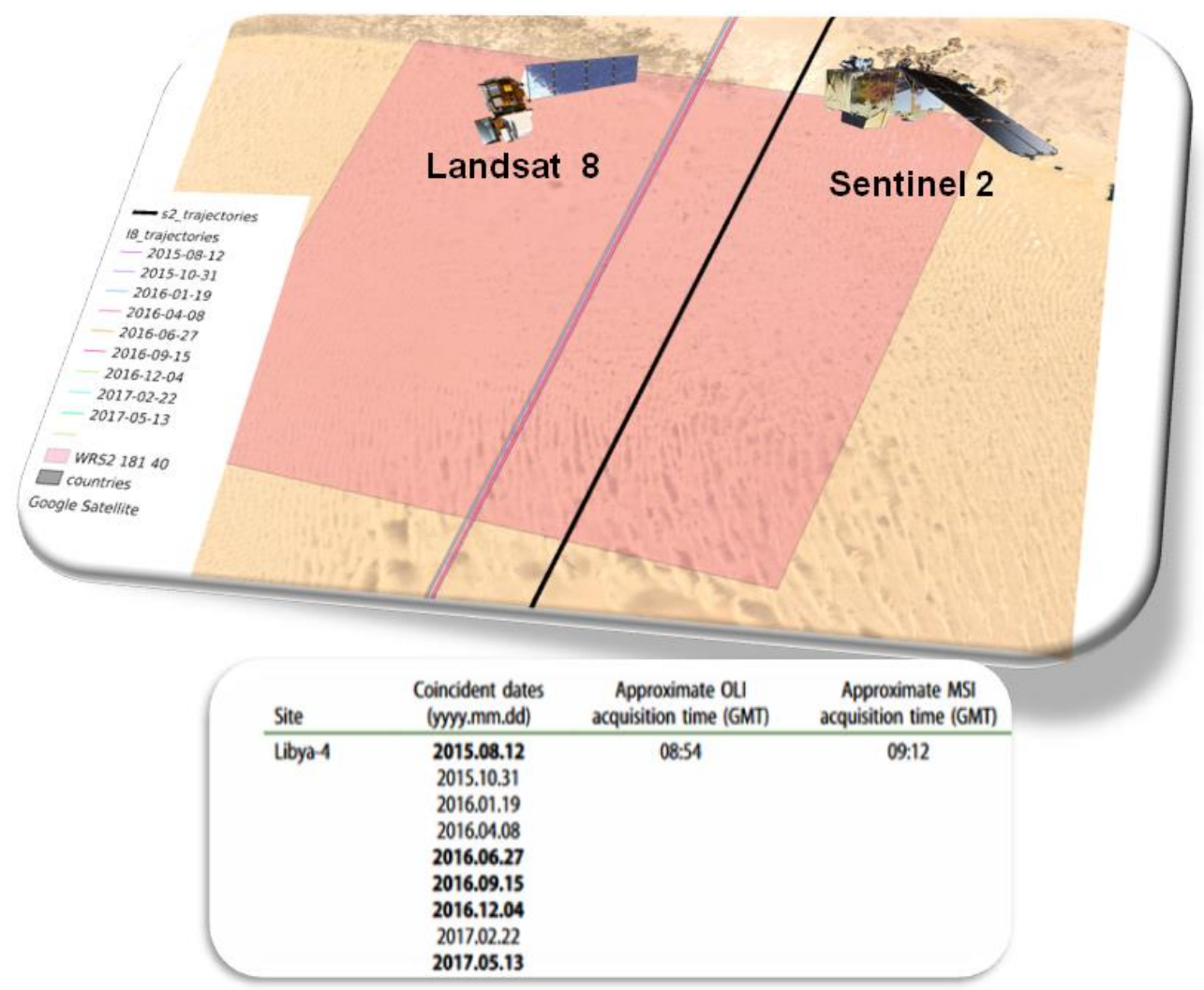

Figura 15. SNOs utilizadas por Barsi et al., (2018) sobre Libya-4. Fuente: Adaptado de Barsi et al., (2018)

Este tipo de metodología de cross-calibration mediante SNO sobre PICS tiene ciertas desventajas, como son las limitaciones en el rango de valores de reflectancia o radiancia utilizados en la comparativa, alta latencia de adquisición sobre los PICS y ausencia de un método standard de definición de la superficie de SNO utilizada para la comparativa.

Con la finalidad de minimizar o eliminar las desventajas de las anteriores técnicas de cross-calibration mediante SNOs, en este capítulo 2 se presenta una metodología global de SNOs para los satélites S2A, S2B y L8. Esta metodología no necesita de un propagador de órbitas y es aplicable sobre cualquier tipo de objetivo espectral a partir de áreas homogéneas. Las SNOs seleccionadas a partir de un criterio de cruce de órbitas y las áreas elegidas mediante un criterio de homogeneidad espacial basado en el CV, permiten obtener resultados inmediatos sobre la comparativa entre sensores que facilite la armonización e interoperabilidad de sus datos. La elección de estos sensores se basó principalmente en las analogías a nivel de resolución espacial y de RSRs entre sus bandas, así como en el gran interés de la comunidad de usuarios de EO en su uso dual (Helder et al., 2018; Stumpf et al., 2018). Los resultados obtenidos mediante esta metodología muestran unos excelentes datos de $R^{2}$ entre $L 8$ con respecto a S2 A/B, con 
valores por encima de 0.98 para todas las bandas a estudio a excepción de la banda NIR ancha de S2 (banda 8), la cual presenta ligeramente una mayor incertidumbre en la comparativa entre sensores con valores en el rango de $R^{2}=0.97-0.98$.

La metodología propuesta en este capítulo 2 debido a su agilidad, flexibilidad y excelentes resultados obtenidos, puede ser un método alternativo o complementario al resto de metodologías mencionadas en esta tesis doctoral.

\subsection{Materiales y Métodos}

\subsubsection{Satélites, sensores y datos utilizados}

Los satélites y sensores utilizados para esta metodología fueron L8-OLI, S2A-MSI y S2BMSI. La elección de los mismos está fundamentada es su excelente calidad contrastada y en la demanda por parte de los usuarios de metodologías de armonización que permitan su uso combinado (Claverie et al., 2018; Helder et al., 2018; Stumpf et al., 2018). También fue esencial en la utilización de los mismos, las similares características a nivel espectral y de resolución espacial de sus bandas, que facilitan la comparativa de resultados (Helder et al., 2018; Stumpf et al., 2018).

Debido a que L8-OLI fue lanzado (febrero de 2013) previamente a la constelación S2, el inicio del periodo de estudio entre los sensores viene limitado por la fecha de lanzamiento del primer satélite de S2, S2A-MSI (junio de 2015). El rango temporal de datos a estudio se fijó entre finales del 2015 hasta prácticamente la terminación del año 2019, exactamente entre el 04/07/2015 hasta el 05/11/2019.

Los datos utilizados para ambos sensores son datos de reflectancia TOA correspondientes a los productos L1C para el caso de S2 y los productos L1TP de la Collection 1 Tier 1 para el caso de L8. Para ambos casos, los productos se encuentran orto-rectificados en la proyección UTM/WGS84.

Todos los productos y datos utilizados tanto para la comparativa de reflectancias, como para la generación de las geometrías de SNOs fueron descargados de los sitios web oficiales de las misiones, para el caso del L8 del USGS (https://earthexplorer.usgs.gov/) y para el caso de S2 del Copernicus Open Access Hub (http://scihub.copernicus.eu).

\subsubsection{Determinación de la trayectoria terrestre de los satélites}

En esta metodología global de SNOs, no se hace uso de ningún tipo de propagador de órbitas. La generación de las trayectorias terrestres y la determinación del tiempo asociada a las mismas a partir de la información de telemetría procedente del satélite, permite una precisión mucho mayor que la que proporciona cualquier modelo de propagación de órbitas. Debido a las diferencias de información disponibles en los metadatos de $\mathrm{L} 8$ y S2, el proceso de generación de las trayectorias terrestres y sus tiempos asociados fue ligeramente diferente. Mientras que la información procedente de los metadatos en los productos L1TP de L8 era lo suficientemente amplia para su 
generación (USGS, 2016), para el caso de S2 A/B fue necesario la descarga de las trayectorias relativas para un ciclo completo (143 órbitas) con un paso de tiempo de 10 segundos (ESA, 2020a) publicadas en la web oficial de la ESA (https://sentinel.esa.int/web/sentinel/missions/sentinel-2/satellite-description/orbit). Para evitar posibles errores asociados a la utilización de diferentes proyecciones geométricas, todos los cálculos fueron realizados en el modelo de proyección tridimensional de la Tierra WGS-84.

L8 realiza maniobras de ajuste de la inclinación del satélite con una frecuencia anual y de manera constante, maniobras de arrastre denominadas Drag Make-Up (DMU) para mantener la trayectoria terrestre dentro de unos límites establecidos (USGS 2020; NASA, 2020a) (Figura 16). Las maniobras DMU permiten ajustar la altitud de la órbita modificando el semieje mayor de la misma. Esta operación permite contrarrestar los posibles efectos de la resistencia atmosférica en el satélite y mantener su circulación orbital, así como preservar de una forma estricta las limitaciones de su trayectoria terrestre (Ruf et al., 2018; USGS 2020; NASA, 2020a; NASA, 2020b). Para L8, el rango de error permitido en la trayectoria de tierra está establecido entre 0-3 Km (NASA, 2020b). Este umbral permite cumplir con la calidad y niveles de tolerancia de incertidumbre definidos para la misión L8.

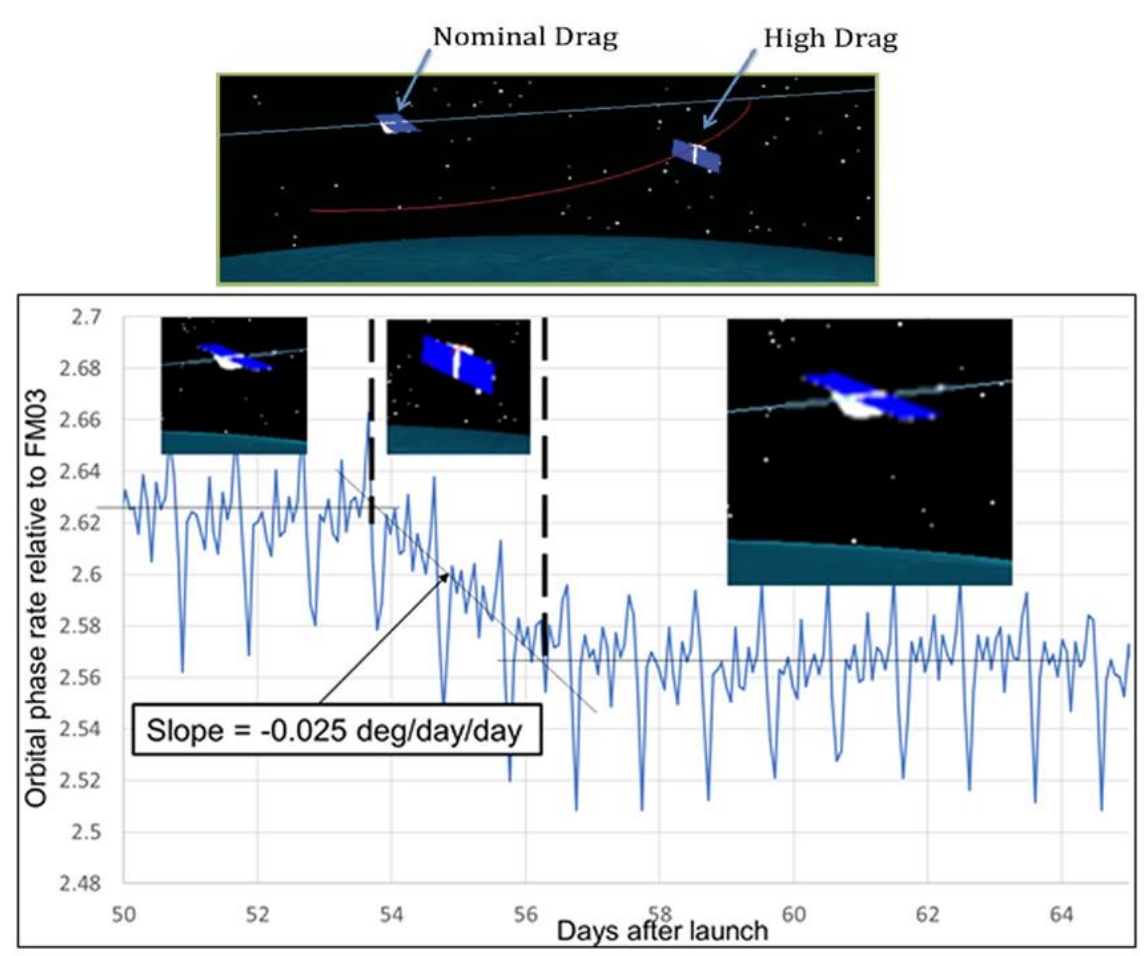

Figura 16. Ejemplo de maniobra High Drag para la constelación de satélites CYGNSS. Fuente: Adaptado de Ruf et al., 2018.

Para el data set de L8 utilizados para este estudio, prácticamente la totalidad de los metadatos de los productos L1TP muestran valores de ángulo de roll entre las mismas 
orbitas inferior a los $2 \times 10^{-3}{ }^{\circ}$. Sin embargo solo un $0.2 \%$ de los mismos, presentan valores más elevados, siendo el máximo valor de ángulo de roll encontrado $4 \times 10^{-3}{ }^{\circ}$, lo que representa una distancia de entre $1.4-2.8 \mathrm{~km}$ fuera del nadir en la trayectoria de tierra. Estos valores encontrados se encuentran dentro del rango de valores establecido para la misión L8 (NASA, 2020b).

La constelación S2 A/B también realiza de manera constante operaciones de mantenimiento de órbita, con un error de tolerancia entre $\pm 2 \mathrm{Km}$ (ESA, 2020a).

Para la determinación de la trayectoria de L8 se hizo uso del footprint de cada imagen, junto con el valor espacial y temporal del centro de cada escena. Mientras la localización del centro de la escena de L8 viene recogida en los metadatos (USGS, 2016), el cálculo del tiempo del centro de la escena se realizó como el tiempo medio entre el inicio y final de la adquisición. La unión de esos puntos permitió generar las órbitas completas de L8 (Figura 17).

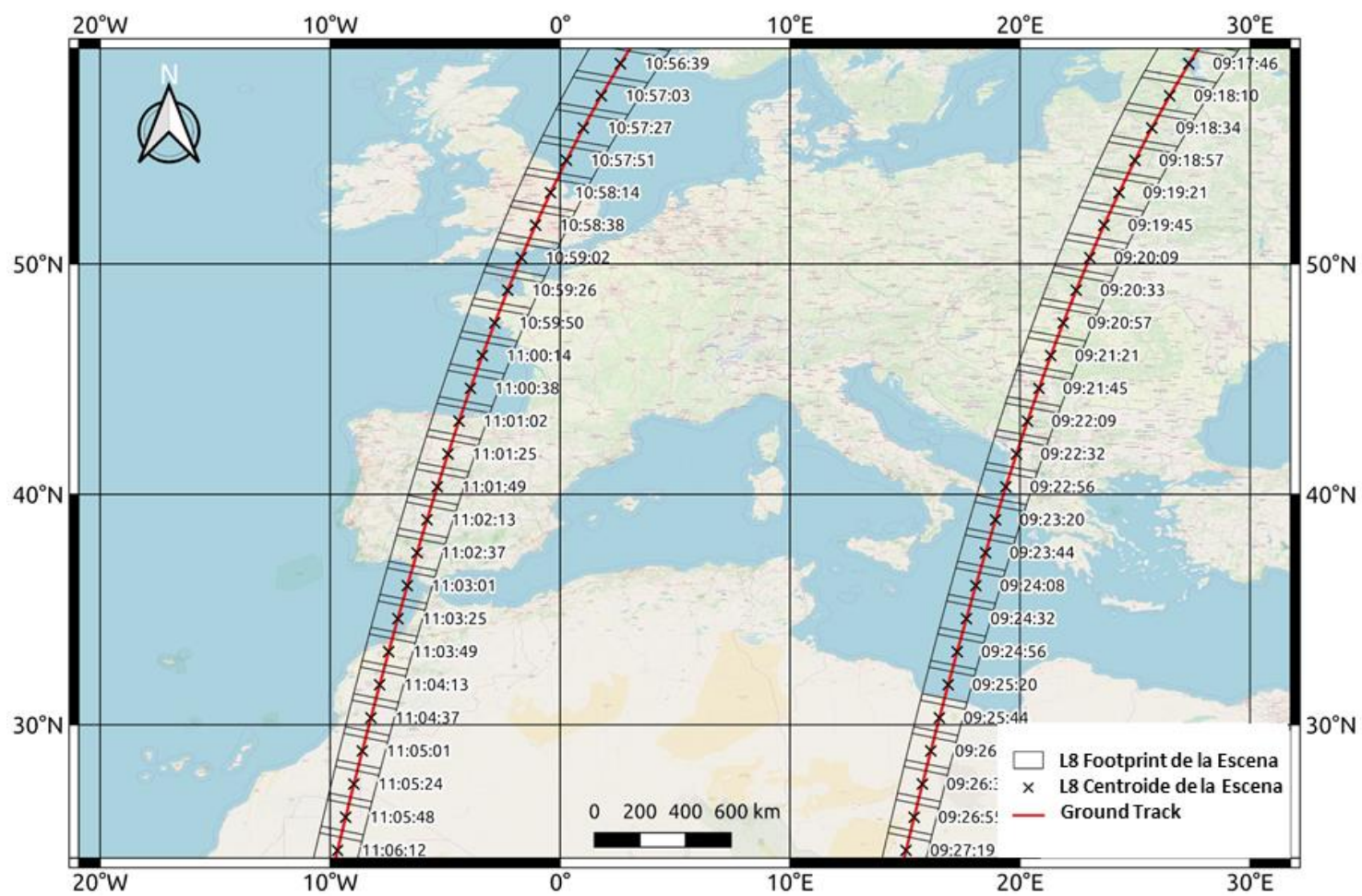

Figura 17. Ejemplo de generación de la trayectoria de tierra para L8 a partir de la información de los footprint y del centro de la escena.

A diferencia del sistema de tilling Worldwide Reference System - 2 (WRS-2) de L8 donde las órbitas del satélite coinciden con la escena, las imágenes de S2 A/B están referenciadas al sistema Military Grid Reference System (MGRS) que no tiene relación con las órbitas de los satélites. Para S2 A/B se hizo uso de los archivos proporcionados por ESA sobre las órbitas relativas del ciclo completo de la constelación, 143 órbitas (ESA, 2020a). Por esa razón, no fue necesario generar la órbita a partir de la información 
de los metadatos, en cambio, si se utilizaron para obtener información temporal y espacial, junto con los footprint de cada imagen para el cálculo del punto de cruce entre órbitas (Figura 18).

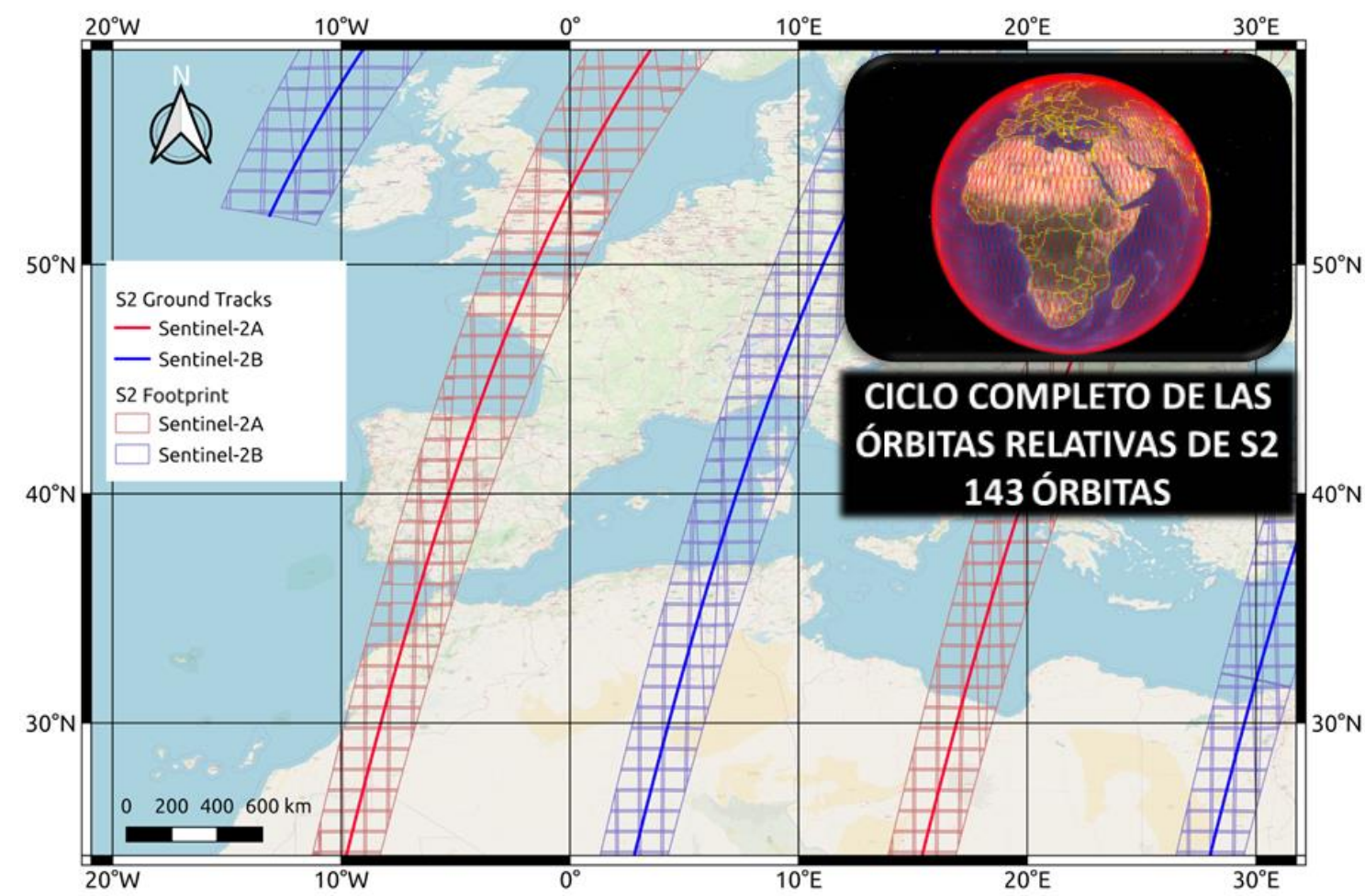

Figura 18. Ejemplo de la trayectoria de tierra para $S 2 A / B$ a partir de la información de las órbitas relativas proporcionadas por ESA y los footprint de los productos L1C referenciados al sistema MGRS.

\subsubsection{Definición y localización de las SNOs}

Se puede definir la SNO ideal como el proceso que ocurre cuando dos o más sensores de satélite observan en el mismo instante el mismo punto nadir de la superficie terrestre bajo las mismas condiciones de iluminación y de visión. Es decir, las SNOs vienen definidas en base a dos criterios fundamentales como son, el temporal (simultaneidad) y el espacial (cruce de órbitas). Debido a la baja probabilidad de encontrar sensores que cumplan la idealidad de este tipo de SNO de manera estricta, estos criterios tienen diferentes rangos de tolerancia para una implementación práctica.

Siguiendo el fundamento de la SNO ideal en la metodología presentada en este capítulo e implementada para los satélites L8-OLI y S2 A/B-MSI, se establecieron criterios temporales y espaciales que permitiesen definir de una manera clara la SNO. El primer criterio que define las SNOs en esta metodología es el espacial, ya que es necesario que las trayectorias terrestres de los sensores a comparar se deban intersecar. Ese punto de intersección nadir para ambos satélites, es el punto de partida para todo el método. El criterio temporal será la diferencia de tiempo que se establezca como umbral para el 
paso de los sensores a estudio por ese punto nadir ya fijado. El tamaño del área de estudio de la SNO, vendrá definido por un área circular cuyo centro se fija en el punto nadir de intersección de las trayectorias terrestres y con un radio variable para cada SNO, en base a un criterio de intersección de las geometrías de sus tiles. Es importante señalar, que las variaciones en los criterios espaciales relacionados con el radio del área circular a estudio tienen un mayor impacto en los ángulos de visión de los sensores, mientras que las variaciones en los criterios temporales influyen especialmente en los ángulos solares de la escena para cada uno de ellos.

Para el caso de L8 y S2 A/B, se obtuvieron los puntos de intersección de las trayectorias terrestres de cruce entre L8 con cada uno de los satélites S2 y se fijó de manera inicial un intervalo temporal de 24 horas para poder evaluar el número de muestras encontradas. Se obtuvieron 1397 SNOs para S2A y 953 para S2B con respecto a L8. Debido al elevado número de SNO localizadas y a la amplia tolerancia temporal establecida que podría tener un impacto significativo en las condiciones atmosféricas de los datos, se restringió el intervalo de tiempo a 30 minutos. También se establecieron restricciones de nubosidad fijando un umbral de $5 \%$ en las escenas S2. No se estableció un umbral más restrictivo debido a que la presencia de nubosidad no tiene porqué estar localizada en el área de estudio. Además, al fijarse el mismo sobre la escena S2 la cual es más pequeña que la de $L 8$, el criterio de limitación de nubosidad es bastante estricto al restringirse inicialmente la zona de aplicación. Una vez aplicados estos criterios con el objetivo de reducir posibles incertidumbres que podrían afectar a la calidad de la muestra, se obtuvieron 96 SNOs de S2A y 60 SNOs de S2B con L8 (Anexos: Productos S2 y L8 utilizados)

Fijados el punto de cruce y el intervalo de tiempo, es necesario fijar el radio del área de estudio de la SNO para definirla completamente. El radio se estableció como la distancia mínima desde el punto de intersección nadir de las trayectorias terrestres entre sensores al polígono resultante de la intersección entre los tiles de L8 y S2 (Figura 19). Debido a que las SNOs están limitadas a la superficie terrestre, en los casos particulares en que las SNO se produzcan en las fronteras de las líneas de costa, el valor del radio del área de la SNO está también limitado por las mismas para evitar grandes masas de agua en el análisis (Figura 20). 


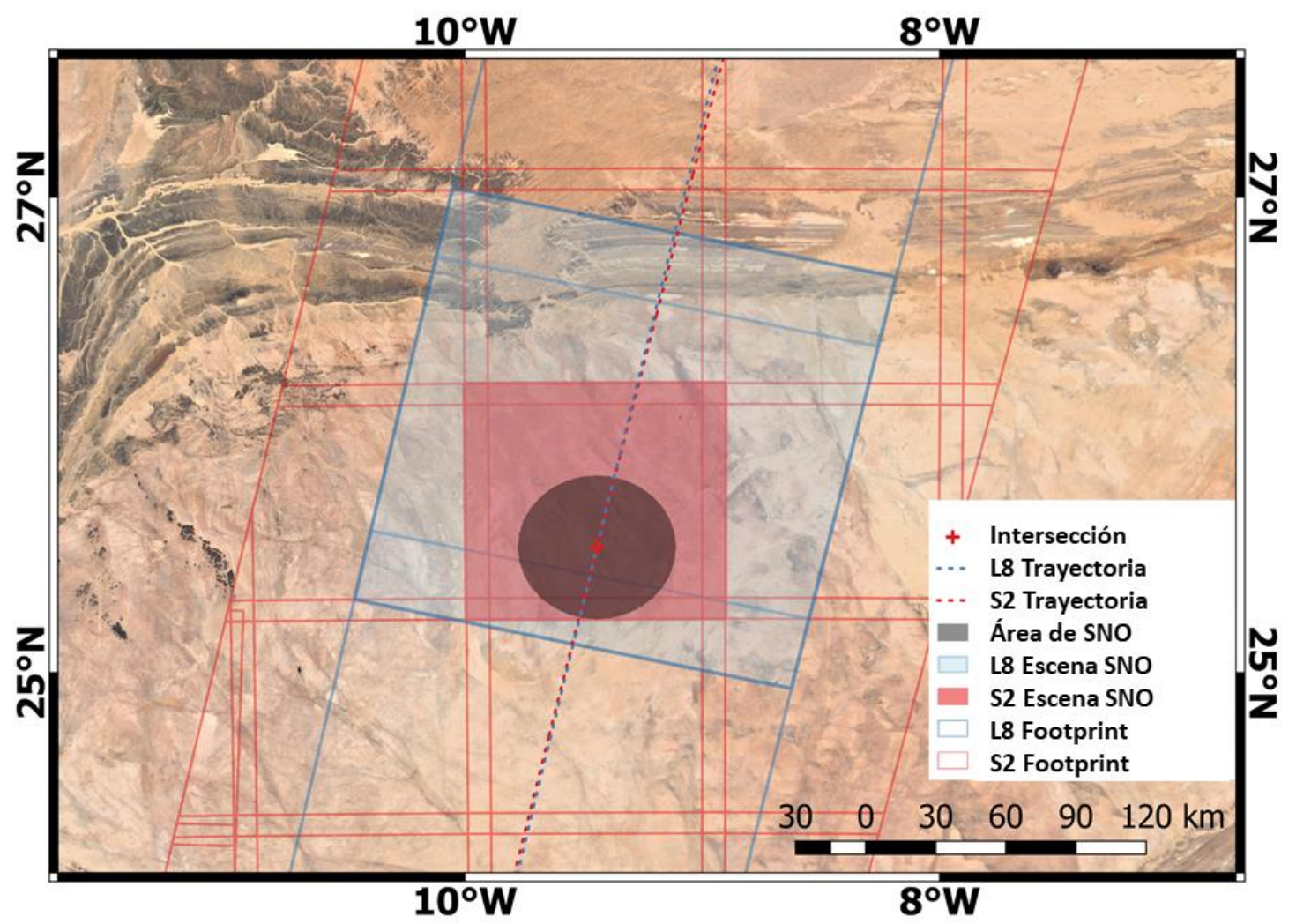

Figura 19. Ejemplo genérico del cálculo del área de la SNO.

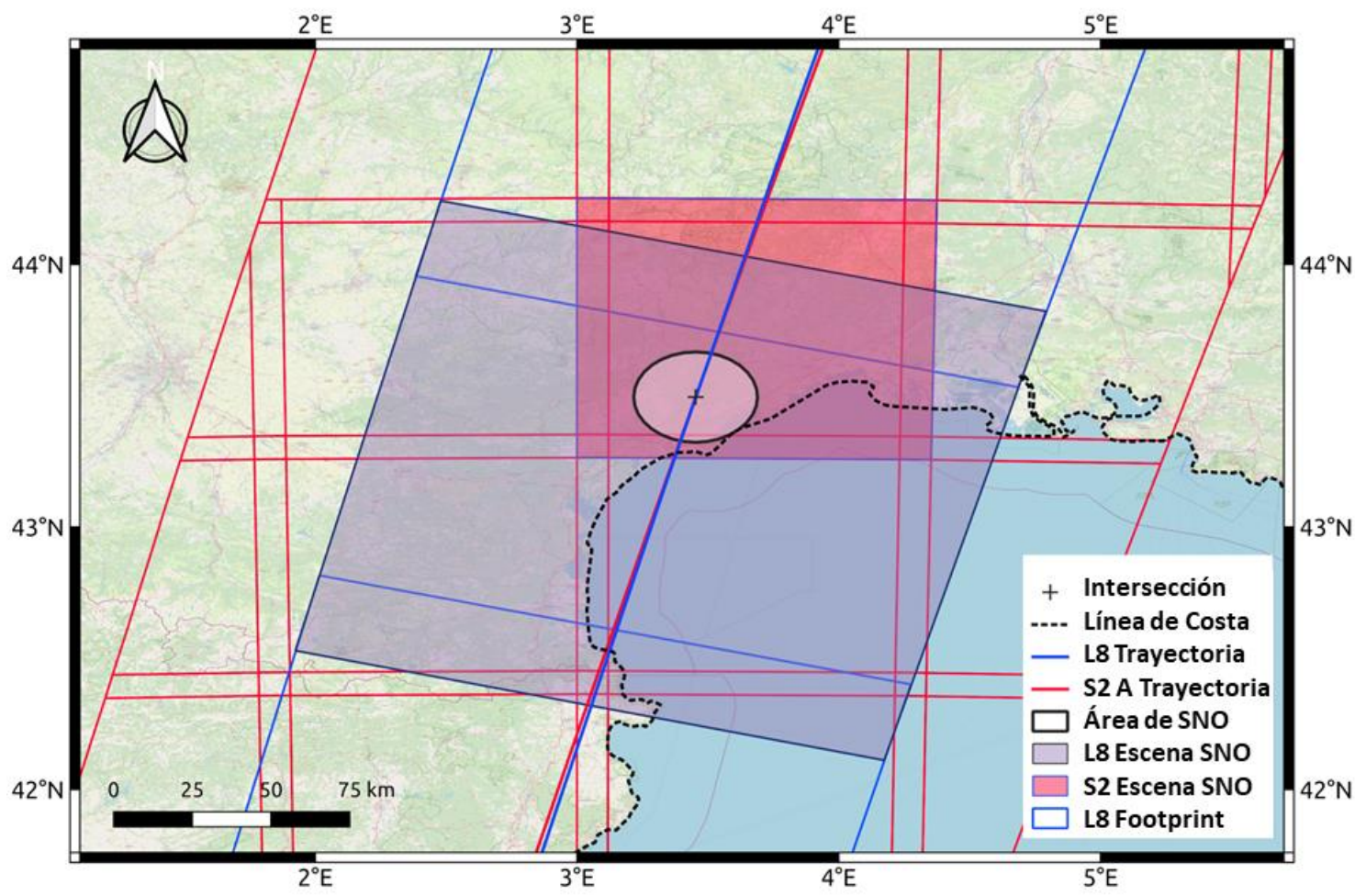

Figura 20. Caso particular de limitación del radio del área de la SNO por la costa del Golfo de Lyon (Francia). 


\subsubsection{Bandas espectrales utilizadas y extracción de datos}

Para esta metodología de SNOs, fueron utilizadas todas las bandas de S2-MSI del rango espectral del infrarrojo cercano visible (VNIR) y el infrarrojo medio (SWIR). Se excluyeron de la comparativa la banda 9 de S2-MSI (vapor de agua) debido a que no existe una banda equivalente en el sensor L8-OLI (Lillesand et al., 2015), así como la banda 10 (cirros) de S2-MSI al ser necesarios datos de la superficie terrestre y no proporcionar esta banda datos útiles de la misma (Gascon et al., 2017).

Como se explica de forma pormenorizada en las siguientes secciones de este capítulo, a partir de la localización de las zonas homogéneas en las bandas de S2 A/B-MSI, se utilizaron esas mismas áreas para L8-OLI, obteniéndose los valores de reflectancia TOA en ambos sensores para cada área de forma individual. A parte de los datos de reflectancia TOA, también se obtuvieron otros datos para su posterior análisis:

$\checkmark$ Distancia al nadir

$\checkmark$ Distancia a la intersección de las trayectorias terrestres

$\checkmark$ Área de la SNO

$\checkmark$ Ángulo de elevación y acimut solar en el área del centroide de la SNO.

El análisis de la comparativa de los valores de reflectancia TOA de cada una de las SNO se realizó de manera individualizada para cada par de bandas del mismo rango espectral de S2-MSI y L8-OLI. En la Figura 21 se muestran las RSRs de los pares de bandas de S2 A/B-MSI y L8-OLI seleccionadas para esta metodología.

Es importante señalar que S2A y S2B, a pesar de tener el mismo sensor MSI, presentan diferencias en las RSRs de las mismas bandas (Figura 21). Incluso dos sensores con el mismo proceso de diseño y de fabricación no son completamente idénticos (Chander et al., 2013). Las diferencias entre las RSRs de las bandas de S2 A/B con respecto a las bandas de $\mathrm{L} 8$ son algo más pronunciadas al ser sensores con diseños distintos. Estas discrepancias entre los sensores MSI y OLI son más visibles en las RSRs de las bandas NIR, red y green de ambos sensores, considerándose sus RSRs muy similares en el resto de bandas (Figura 21). 


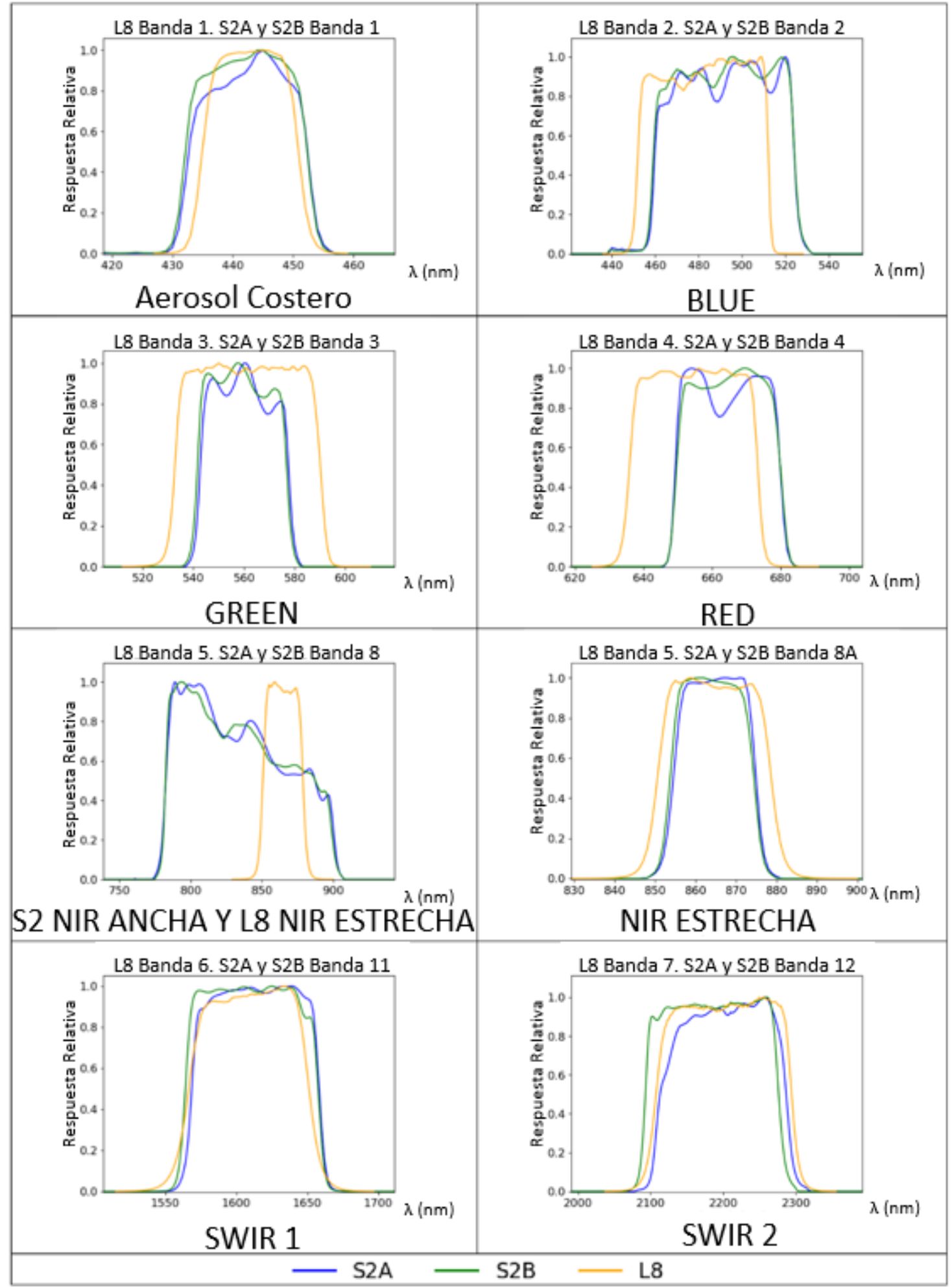

Figura 21. Comparativa de las RSRs de los pares de bandas S2A/B-MSI y L8-OLI elegidos para la metodología de SNOs. 


\subsubsection{Uniformidad espacial}

Para poder determinar las zonas homogéneas a comparar dentro del área de estudio definida previamente por las SNOs, es necesario utilizar una métrica de homogeneidad que facilite la localización de dichas zonas. EI CV es un operador utilizado por varios autores para medir la homogeneidad espacial (Kneubühler et al., 2006; De Vries et al., 2007; Gu et al., 1990) y temporal (Helder et al., 2010). Por este motivo, se utilizó este operador, adaptándolo a la metodología de SNOs según las necesidades de la misma. En la metodología de las SNOs, no tiene sentido el estudio temporal al realizarse la adquisición en condiciones de simultaneidad y sobre una única escena. Por tanto, únicamente fue necesario realizar el estudio espacial de las escenas de las SNOs para las bandas de S2. La utilización del sensor S2-MSI como referencia en vez de L8-OLI, es debido a la mayor resolución espacial del primero, permitiendo una mejor discretización de la uniformidad espacial de la imagen.

\subsection{6. Áreas homogéneas}

Una vez realizado el análisis y generación de las imágenes de uniformidad espacial del área de estudio a partir de las bandas de S2 y del operador CV, era necesario localizar las áreas más homogéneas para su posterior comparativa con los datos L8. Para ello, se fijó un umbral a partir del valor de CV correspondiente al percentil 1 de la muestra. Una vez aplicado el umbral, se realizó la binarización de las imágenes para diferenciar las zonas seleccionadas como las más homogéneas de las que no. Para evitar píxeles aislados y mejorar la pureza de la muestra, se procedió a aplicar el operador erosión (Piper, 1985; Liang et al., 1989). Una vez eliminados esos píxeles, se aplicó el operador dilatación (Piper, 1985; Liang et al., 1989) para que los grupos de píxeles afectados por el operador erosión recuperasen parcialmente su tamaño inicial. El kernel utilizado para el operador erosión es mayor que el aplicado al operador dilatación con la finalidad de minimizar los posibles efectos geométricos en las zonas homogéneas entre las bandas de L8 y S2 como consecuencia de sus dispares resoluciones espaciales. Una vez fijadas las áreas, se procedió a la vectorización de las mismas (Figura 22). Este proceso se realizó para cada SNO localizada y banda a estudio de S2 A/B, creándose un conjunto de áreas homogéneas para cada SNO. En la Figura 23 se muestra un ejemplo de las áreas homogéneas localizadas en una SNO, así como las trayectorias terrestres de los satélites y el punto nadir de intersección de las mismas. 


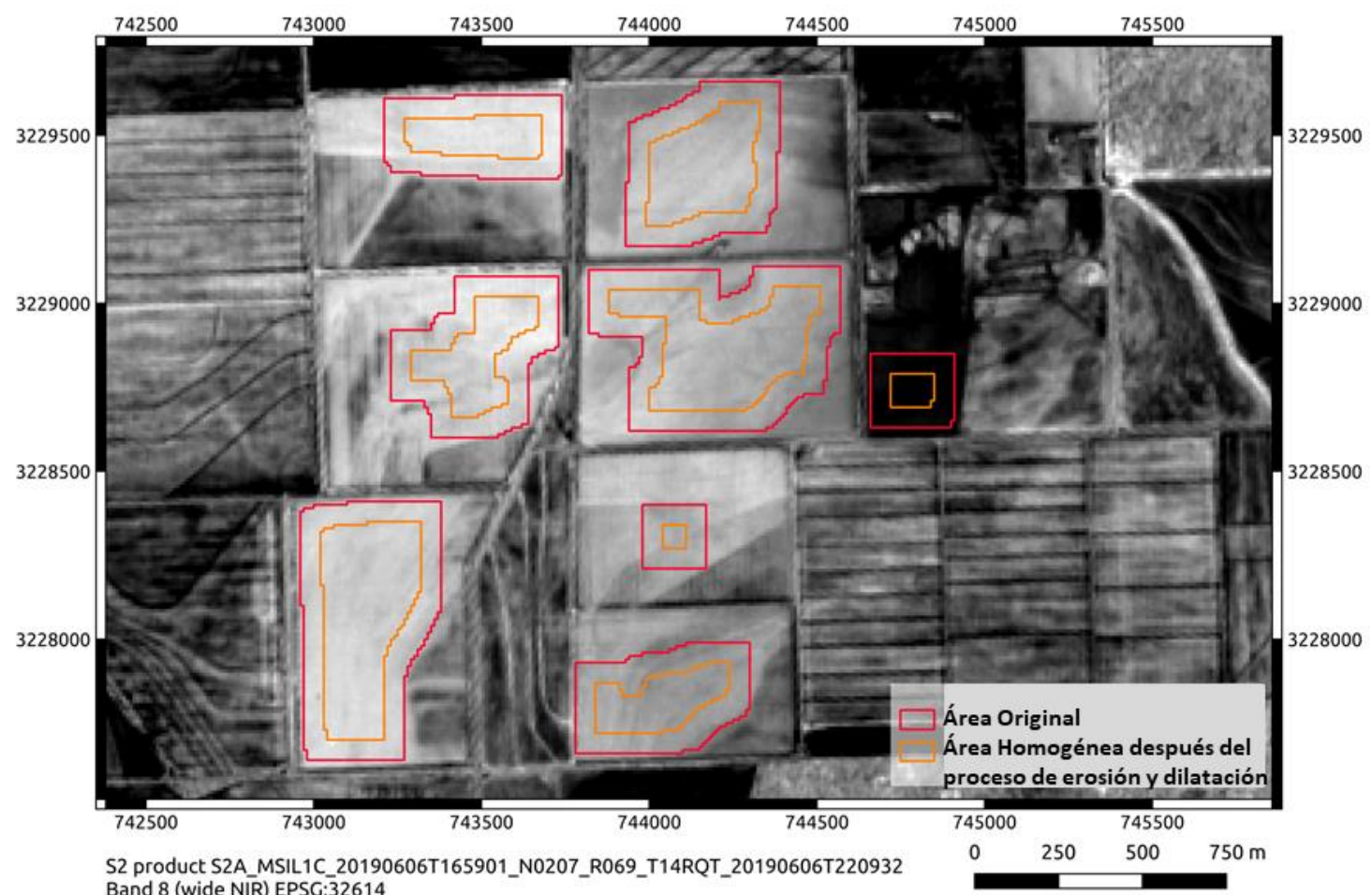

Figura 22. Ejemplo de aplicación de los operadores erosión y dilatación para la generación de las áreas homogéneas.

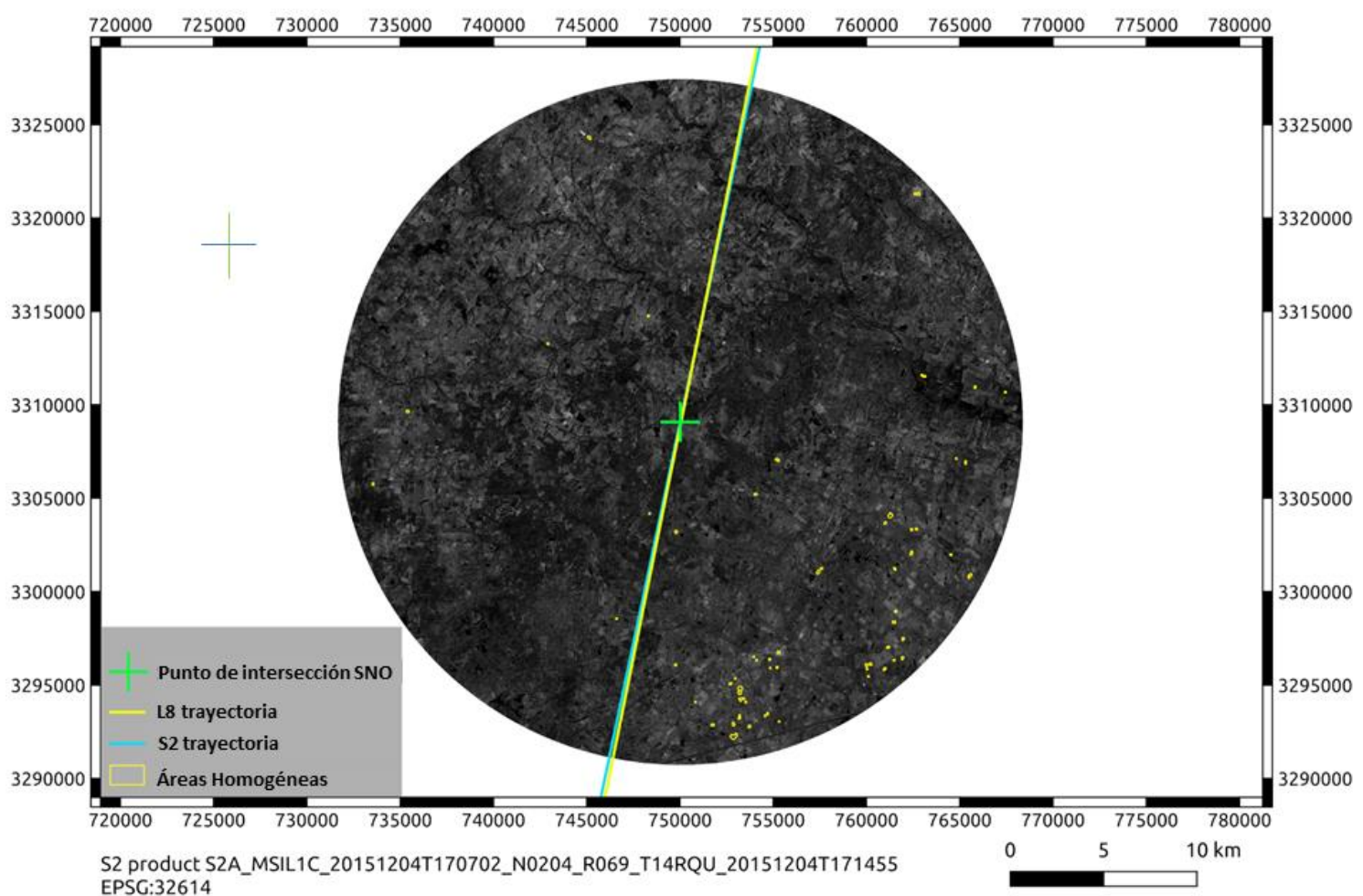

Figura 23. SNO y áreas homogéneas localizadas para la banda NIR ancha de S2. ID del Producto: S2A_MSIL1C_20151204T170702_N0204_R069_T14RQU_20151204T171455. 
La utilización de las bandas de S2 A/B está motivada principalmente porque presentan una mayor resolución espacial con respecto a $L 8$, con la única excepción de la banda de aerosol costero.

El umbral del percentil 1 se fijó después de un proceso de análisis donde se implementaron diferentes valores de percentil en un rango del 0.1 - 5.0, observándose que los resultados no mostraban un gran impacto a las variaciones del valor. El valor umbral del percentil 1 permite un equilibrio entre la selección de zonas altamente homogéneas y con un número de áreas en la muestra lo suficientemente amplio para su posterior análisis y comparativa.

Es importante señalar que el cálculo de las áreas homogéneas se realizó sobre los valores de DN de las imágenes S2. Con el fin de mantener la naturaleza empírica de la metodología, los vectores generados de las áreas homogéneas y no las imágenes son los que se proyectan para la obtención de los datos entre los sensores participantes para cada SNO. También se estableció un umbral mínimo de superficie para las áreas homogéneas, que se aplicó una vez finalizaron los operadores erosión y dilatación. Este umbral se fijó en $900 \mathrm{~m}^{2}$, lo que equivale a 9 píxeles L8-OLI de las bandas multiespectrales. Este umbral permite continuar mejorando la pureza de la muestra utilizada.

Una vez obtenido el vector de áreas homogéneas para cada SNO y banda de los sensores, se realizó la conversión de los valores de DN a valores de reflectancia TOA teniendo en cuenta la influencia del ángulo solar de la escena. Para el caso de S2, no se utilizó un único valor de ángulo solar para toda la escena, sino que el factor de reescalado de los metadatos tiene en cuenta ese ángulo en cada píxel de la imagen (ESA, 2020b). En cambio, para el caso de L8 una vez aplicados lo coeficientes de conversión fue necesario dividir ese valor por el seno del ángulo de elevación solar o por el coseno del ángulo cenital solar (Ecuaciones 6 y 7) calculados mediante la librería de Phyton Pysolar. Esta operación permite corregir el valor de la reflectancia TOA debido al ángulo solar (USGS, 2020a). Es importante destacar, que el ángulo solar utilizado fue el calculado para el centroide de cada área homogénea.

$$
\begin{aligned}
\rho_{\text {Corregida }} & =\frac{\rho_{\text {Sin Corregir }}}{\sin \theta_{\text {Elevación Solar }}} \text { (Eq. 6) } \\
\rho_{\text {Corregida }} & =\frac{\rho_{\text {Sin corregir }}}{\cos \theta_{\text {Cenit Solar }}} \text { (Eq. 7) }
\end{aligned}
$$

\subsubsection{Comparativa entre sensores, análisis de datos y eliminación de outliers.} Una vez obtenidas las áreas homogéneas, se procedió a calcular los valores de reflectancias TOA corregidas de L8 y S2 A/B por el efecto del ángulo solar sobre las mismas. Posteriormente, se realizó la comparativa de reflectancia TOA sobre las áreas homogéneas seleccionadas para cada par de bandas elegidas. Los valores de reflectancia TOA de L8 y de S2 A/B se compararon mediante una regresión lineal. Los datos de todas 
las SNOs se representaron de manera gráfica para la comparativa de las reflectancias TOA de L8 con respecto a las de S2A y S2B de forma individualizada. Esto permite localizar de forma visual los posibles outliers de la comparativa entre sensores, ya que debido a la diferencia de 30 minutos establecida de umbral entre adquisiciones SNO, algunas de las áreas homogéneas podían tener nubosidad, zonas de sombra, áreas nubladas, etc (Figura 24).
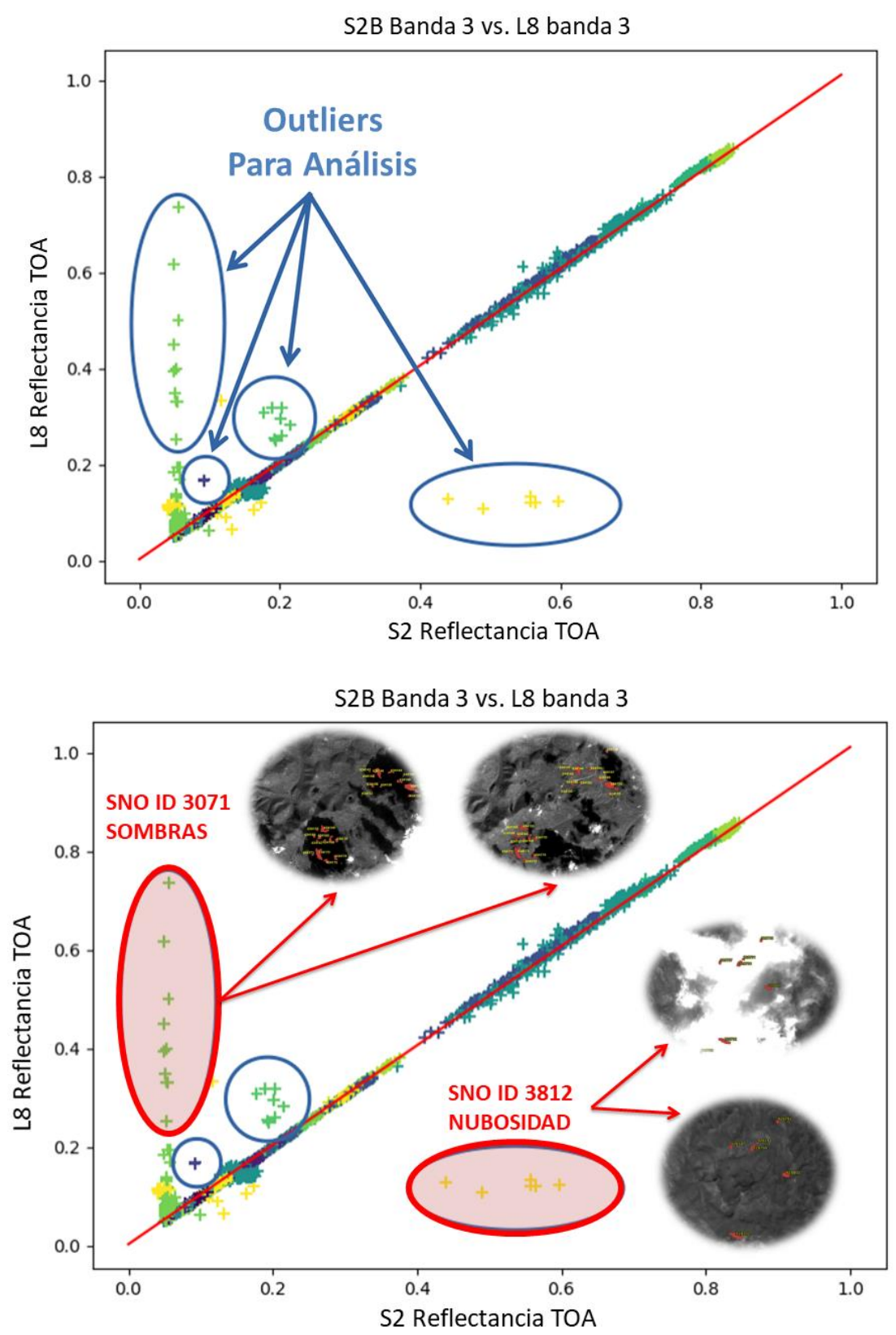

Figura 24. Comparativa de los valores reflectancia TOA para la banda green de L8 y S2. En la misma se aprecian los diferentes outliers encontrados y un ejemplo de outliers por nubosidad y sombras para dos SNO diferentes. Cada punto es un área homogénea diferente, siendo los diferentes colores de las áreas homogéneas las SNOs a las que pertenecen. La línea en color rojo es la regresión lineal de la comparativa entre ambos sensores. 
Los outliers provocados por la presencia de efectos atmosféricos visualmente perceptibles y las escenas que contienen flujos de agua también fueron eliminados debido a su impacto variable en la radiometría (Figura 25 y Figura 26). Para una mejor localización y posterior análisis de los outliers, cada SNO se representó con una paleta de colores diferente. Todos los outliers localizados pasaron un proceso de análisis antes de la eliminación definitiva de la comparativa.

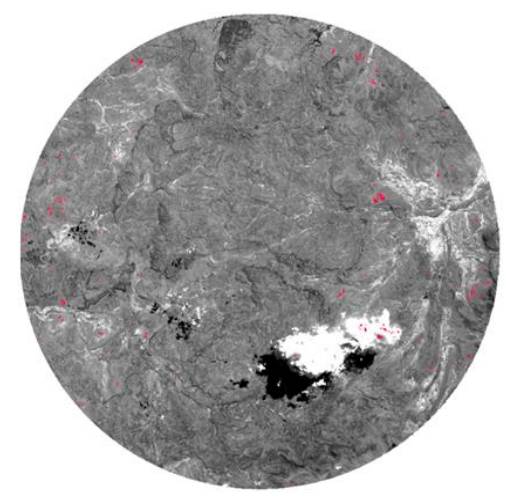

NUBES

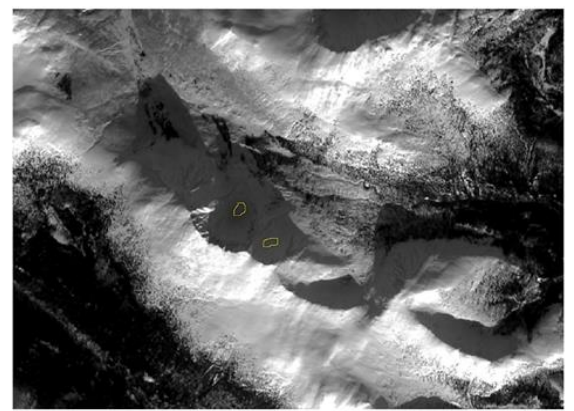

SOMBRAS

Figura 25. Ejemplo de outliers de nubosidad y sombras localizadas sobre las áreas homogéneas después de la inspección visual.

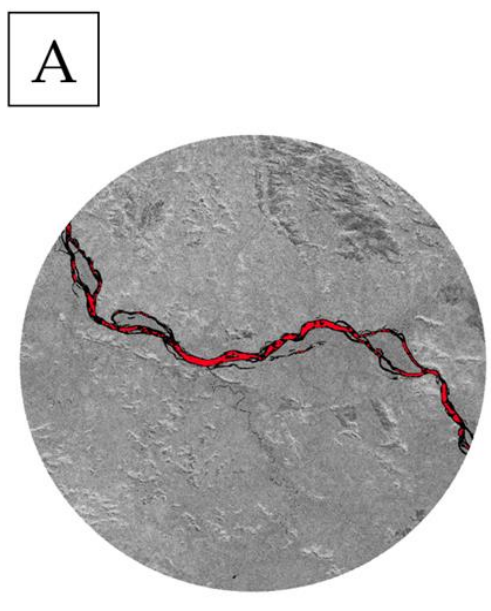

ZONAS DE AGUA

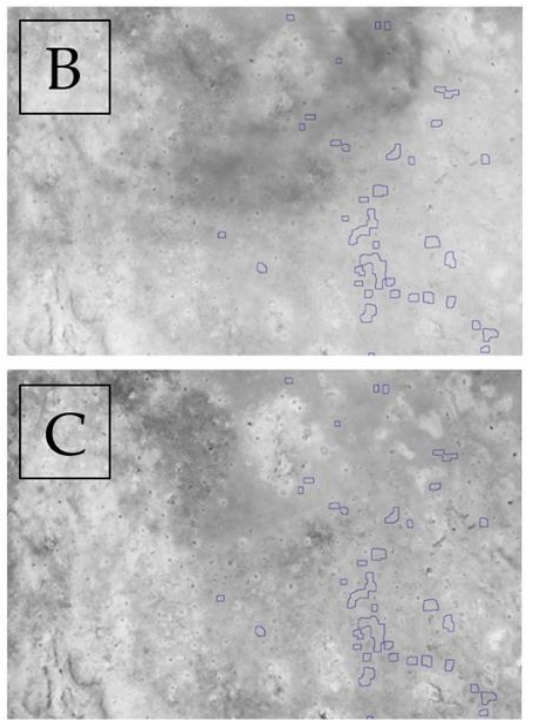

EFECTOS ATMOSFÉRICOS

Figura 26. Outliers identificados durante la inspección visual. Áreas homogéneas en rojo sobre zonas de agua (A). Áreas homogéneas en azul debido a efectos atmosféricos en una SNO para la banda SWIR2 de S2A (B) y $L 8(C)$.

Con el propósito de eliminar todos los outliers que afectaban a la comparativa, en caso de localizar en alguna banda efectos atmosféricos después de la inspección visual, toda la SNO fué eliminada. 
Otros tipos de outliers que se encontraron durante el análisis, fueron efectos de la BRDF en laderas con presencia de nieve (Figura 28). Este tipo de factores, fueron localizados y eliminados de forma ágil, debido a los valores de reflectancia TOA superiores a 1 que mostraban los sensores en esas zonas. También se localizaron outliers en la comparativa cuya causa eran penachos de humo que afectaban a las zonas homogéneas a estudio (Figura 27).

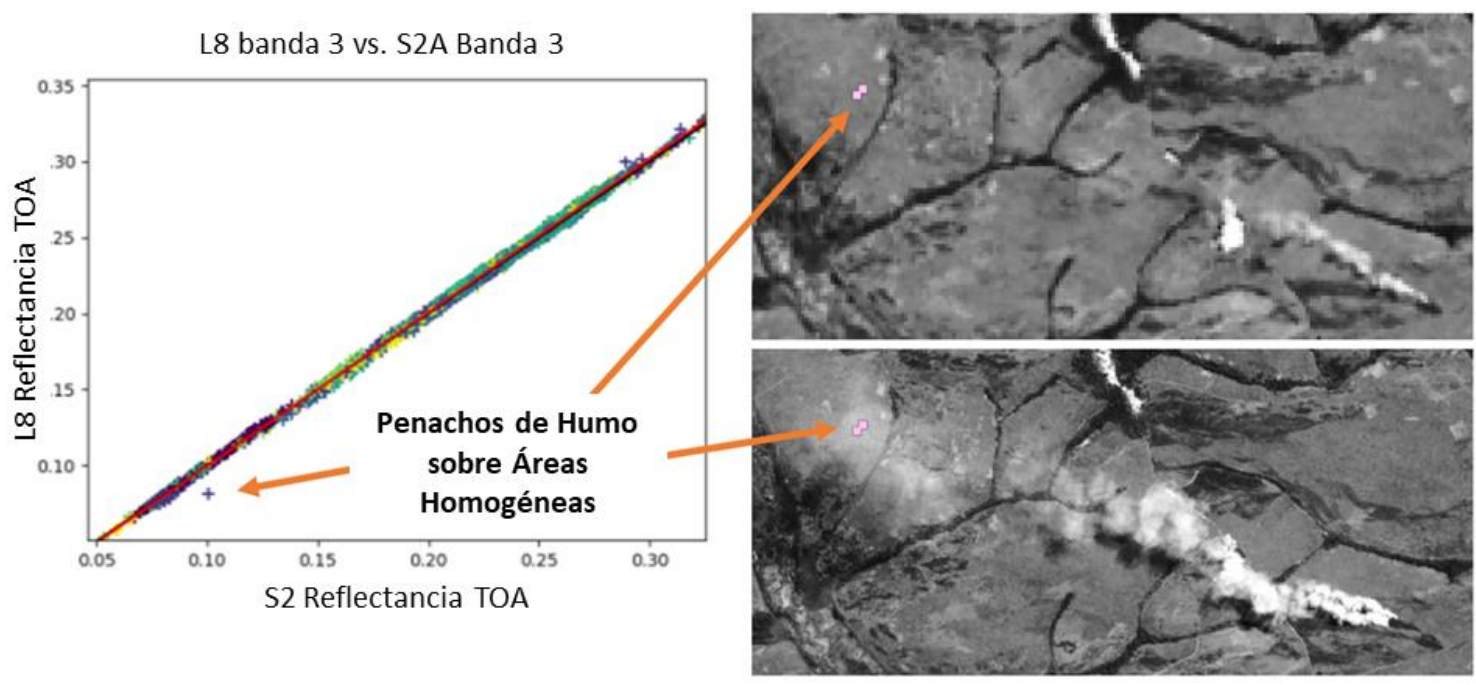

Figura 27. Áreas homogéneas afectadas por penachos de humo.
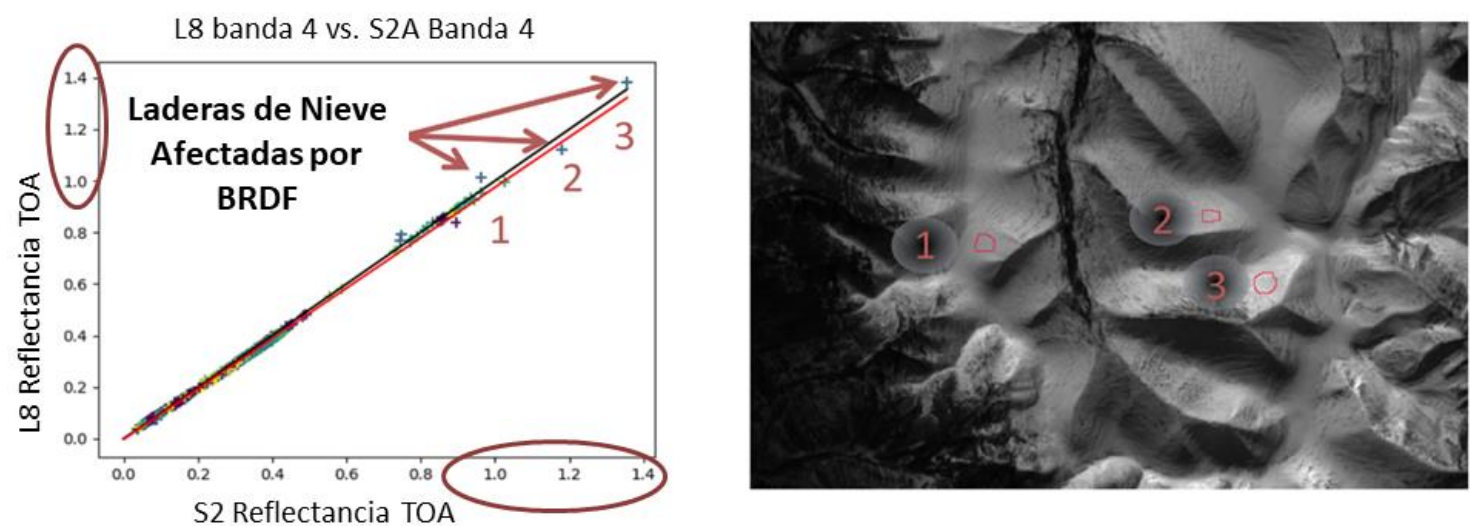

Figura 28. Valores de reflectancia por encima de 1 como consecuencia de los efectos de BRDF provocados por la anisotropía de la nieve en las laderas de una zona montañosa.

De forma general la inspección visual de los outliers es un proceso ágil tanto en su localización, como en su posterior análisis y verificación, al identificarse de una forma clara la localización de los mismos.

\subsubsection{Análisis de las clases de superfices de suelo utilizadas.}

Debido a la naturaleza global de la metodología, existe una gran variedad de superficies utilizadas en el análisis, por ello, era importante conocer la ponderación de cada una de ellas en el conjunto de datos utilizados. Para disponer de esta información, fué necesario 
hacer uso de un land cover global lo más actualizado a nivel temporal y con la mayor resolución espacial posible. La aplicación del land cover sobre las áreas homogéneas, permitió conocer no solo la distribución de las clases de superficie utilizadas y su ponderación, sino el comportamiento de la metodología en diferentes superficies con características espectrales muy dispares.

Después de un pormenorizado análisis de los land cover globales disponibles, se utilizó el Copernicus Global Land Cover Layers collection 2 a 100 m de resolución espacial (CGLSLC100) (Buchhorn et al., 2019) debido a su resolución espacial y al data set actualizado (Figura 29).

\section{Copernicus Global Land Cover Layers: CGLS-LC100 collection 2}

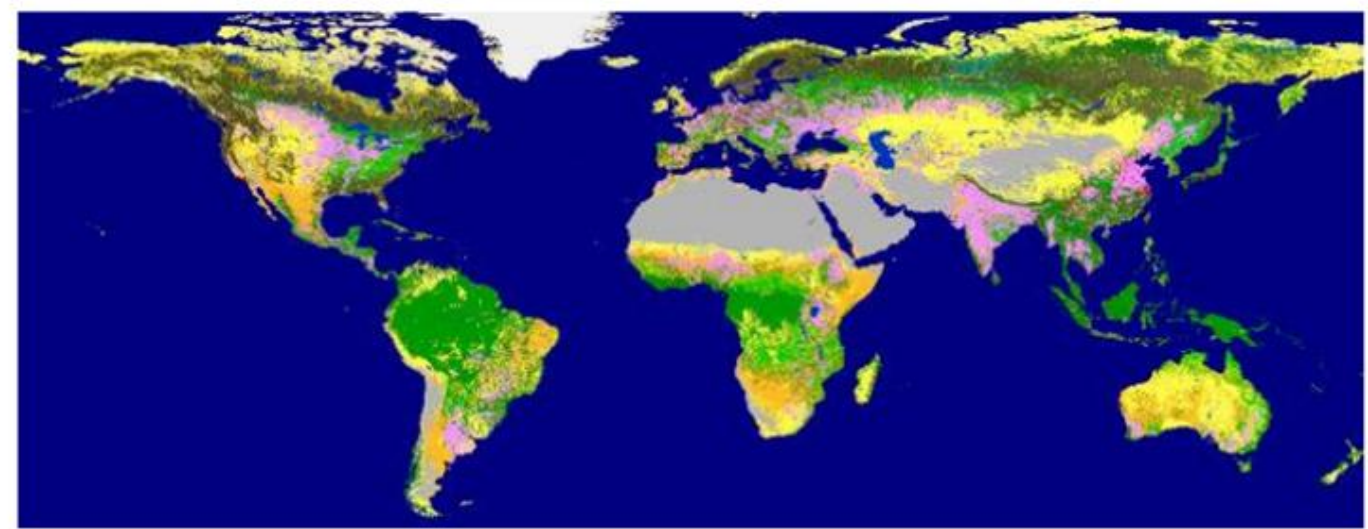

DObservaciones del Satélite PROBA-V para el Año de Referencia 2015 $\square$ Clasificación Discreta con 23 Clases

$\square$ Alta Resolución Espacial: 100 m $\square$ Elevada precisión del Land Cover : $80 \%$

Figura 29. Principales características del CGLS-LC100. Fuente: Adaptado de Buchhorn et al., (2019).

En la Figura 30 se presenta un esquema general del flujo de trabajo de la metodología global de SNOs mediante zonas homogéneas para una mejor comprensión del método. 


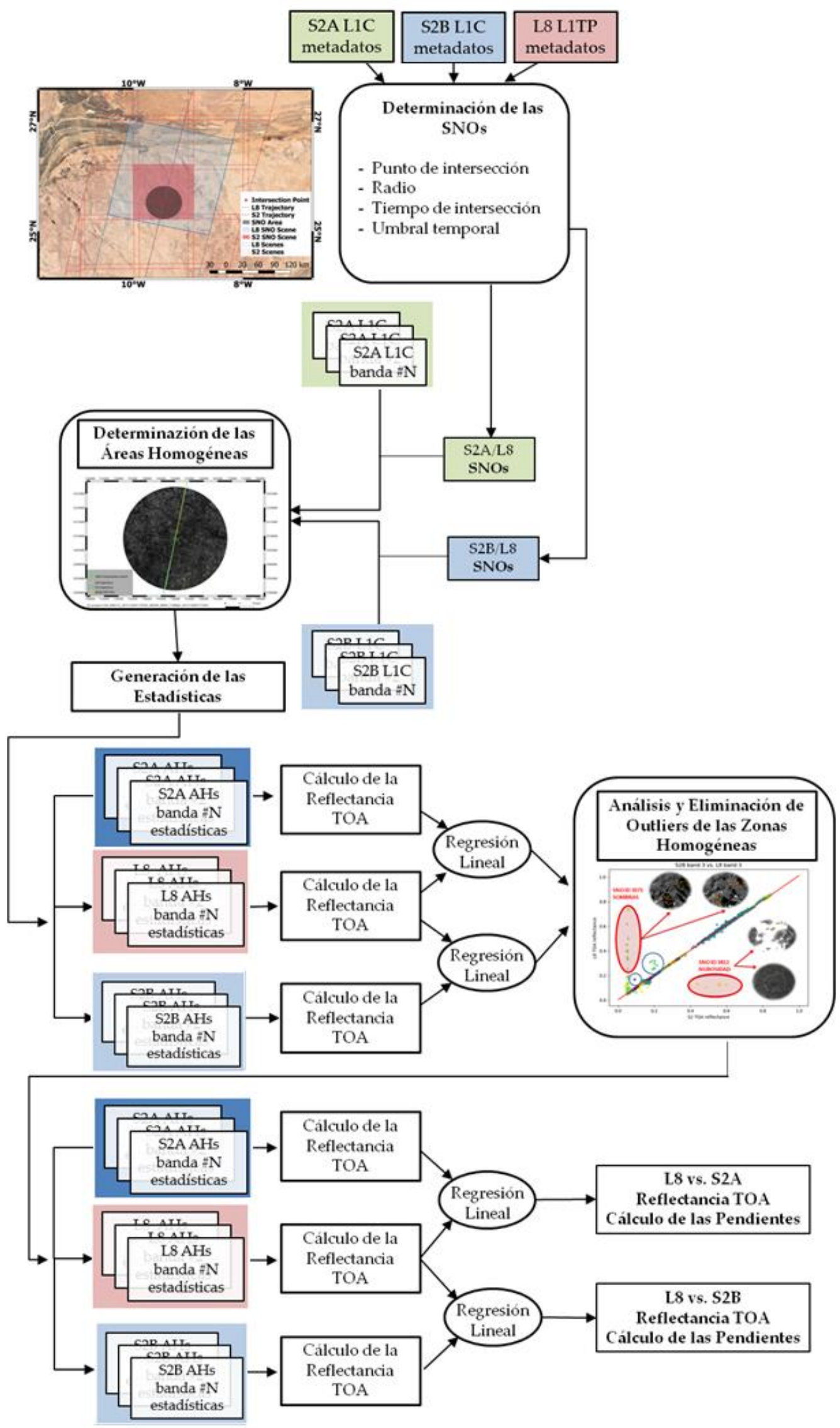

Figura 30. Flujo de trabajo de la metodología de SNOs a partir de áreas homogéneas. 


\subsection{Resultados}

La presentación de los resultados se ha dividido en varias subsecciones para una mejor organización y exposición de los mismos.

\subsubsection{Distribución global de las SNOs localizadas.}

Una vez aplicados los umbrales de intervalo de tiempo de 30 minutos entre adquisiciones y el umbral del $5 \%$ de nubosidad sobre las adquisiciones $\mathrm{S} 2$, el número de SNOs localizadas para el caso de S2A con respecto L8 fueron 96, mientras que para S2B con $L 8$ se encontraron 60. En las Figura 31 y Figura 32 se muestra la distribución mundial de SNOs localizadas para S2A y S2B con L8. Cada círculo en las Figura 31 y Figura 32 es una SNO, siendo el tamaño del área del círculo de cada SNO variable, al estar relacionado con el área de estudio definida para la misma. La paleta de colores hace referencia al intervalo de tiempo entre adquisiciones de la SNO. Para una mejor visualización de las Figura 31 y Figura 32, las áreas circulares de las SNOs se han sobredimensionado.

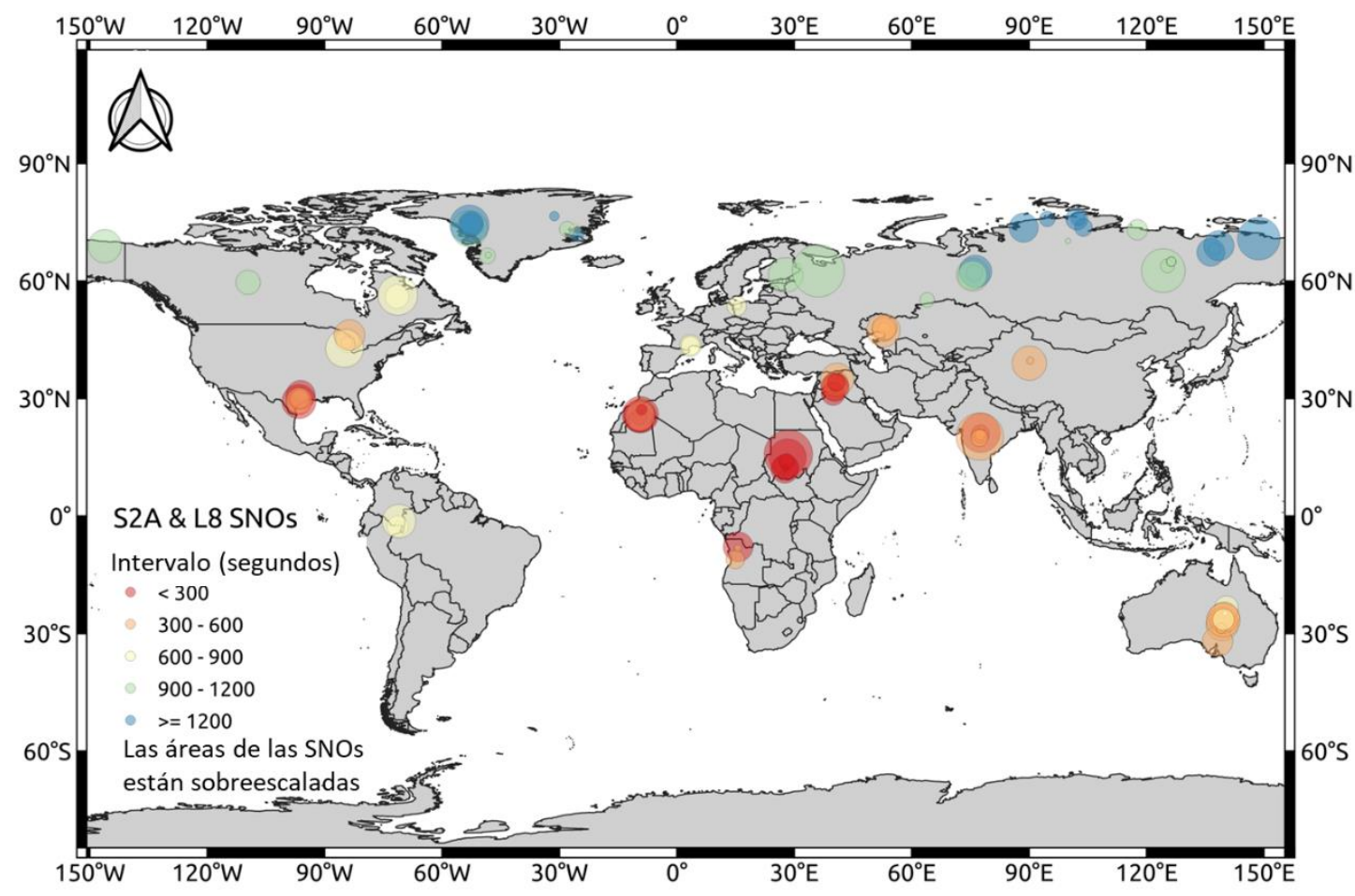

Figura 31. Distribución mundial de las SNOs localizadas entre S2A y L8. 


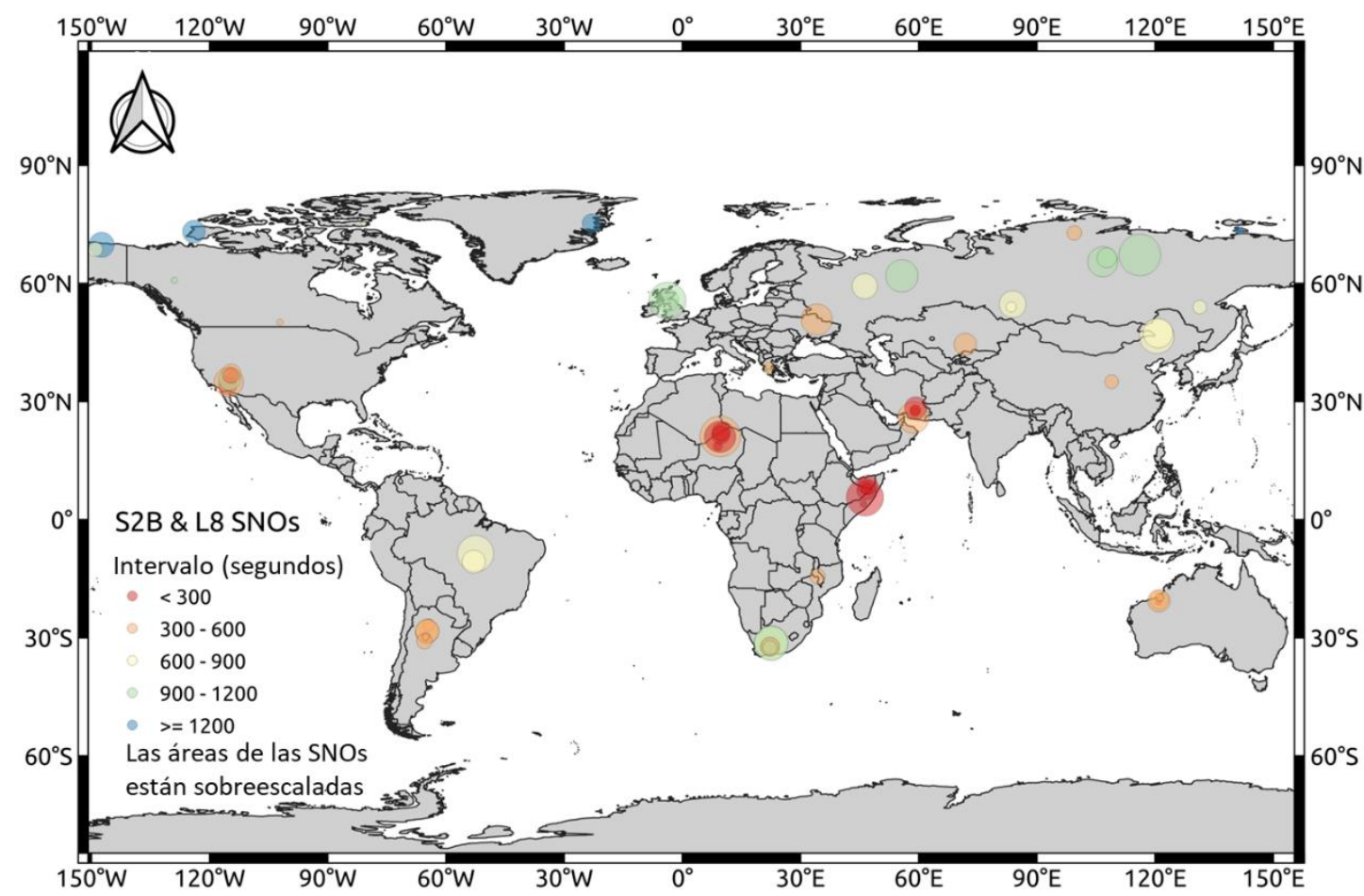

Figura 32. Distribución mundial de las SNOs localizadas entre S2B y L8.

\subsubsection{Comparativa de los valores de reflectancia TOA.}

Para cada par de bandas de S2A-L8 y S2B-L8 del mismo rango espectral, se realizó una regresión lineal de los valores de reflectancia TOA de las áreas homogéneas con los outliers previamente eliminados. También se calculó las incertidumbres de la comparativa para establecer un amplio intervalo de confianza de la medida, para ello se fijó un rango de $\pm 3 \sigma$ de los residuos.

En la Tabla 3 y Tabla 4 se muestran los resultados de la regresión lineal de cada par de bandas de S2A-L8 y S2B-L8, mientras que en la Figura 33 y Figura 34, se muestran los gráficos de la comparativa de las regresiones lineales. Cada punto es un área homogénea diferente, el color de cada área homogénea hace referencia a la SNO a la que corresponde y la línea roja es la regresión lineal.

Los valores obtenidos en la Tabla 3 y Tabla 4 muestran un $R^{2}$ muy elevado de 0.99 . Para el caso del valor de offset, todas las bandas presentan valores absolutos por debajo del $0.38 \%$ a excepción de la banda NIR ancha que presenta una mayor incertidumbre en el rango del $1.4 \%-1.9 \%$. 
Tabla 3. Resultados de la regresión lineal de la comparativa de valores de reflectancia TOA para cada par de bandas de L8 y S2A.

L8 vs. S2A

\begin{tabular}{cccccc}
\hline $\begin{array}{c}\text { Banda } \\
\text { S2 }\end{array}$ & $\begin{array}{c}\text { Banda } \\
\text { L8 }\end{array}$ & $\begin{array}{c}\text { Nombre de } \\
\text { la Banda S2 }\end{array}$ & Pendiente & Intersección & $\mathbf{R}^{\mathbf{2}}$ \\
\hline $\mathbf{1}$ & 1 & $\begin{array}{c}\text { Aerosol } \\
\text { Costero }\end{array}$ & 0.99111 & $-9.307 \mathrm{E}-04$ & 0.99993 \\
\hline $\mathbf{2}$ & $\mathbf{2}$ & Blue & 0.99267 & $3.248 \mathrm{E}-04$ & 0.99907 \\
\hline $\mathbf{3}$ & $\mathbf{3}$ & Green & 1.01095 & $-1.441 \mathrm{E}-03$ & 0.99969 \\
\hline $\mathbf{4}$ & 4 & Red & 0.97671 & $-2.016 \mathrm{E}-03$ & 0.99874 \\
\hline $\mathbf{8 A}$ & 5 & NIR Estrecha & 0.99087 & $-3.732 \mathrm{E}-04$ & 0.99953 \\
\hline $\mathbf{8}$ & 5 & NIR Ancha & 1.02380 & $1.470 \mathrm{E}-02$ & 0.99115 \\
$\mathbf{1 1}$ & 6 & SWIR 1 & 0.98953 & $-2.443 \mathrm{E}-03$ & 0.99956 \\
\hline $\mathbf{1 2}$ & $\mathbf{7}$ & SWIR 2 & 1.00041 & $-3.116 \mathrm{E}-03$ & 0.99964 \\
\hline
\end{tabular}

Tabla 4. Resultados de la regresión lineal de la comparativa de valores de reflectancia TOA para cada par de bandas de L8 y S2B.

L8 vs. S2B

\begin{tabular}{cccccc}
\hline $\begin{array}{c}\text { Banda } \\
\text { S2 }\end{array}$ & $\begin{array}{c}\text { Banda } \\
\mathbf{L 8}\end{array}$ & $\begin{array}{c}\text { Nombre de } \\
\text { la Banda S2 }\end{array}$ & Pendiente & Intersección & $\mathbf{R}^{\mathbf{2}}$ \\
\hline $\mathbf{1}$ & 1 & $\begin{array}{c}\text { Aerosol } \\
\text { Costero }\end{array}$ & 1.00396 & $-9.729 \mathrm{E}-04$ & 0.99991 \\
$\mathbf{2}$ & $\mathbf{2}$ & Blue & 1.01424 & $-8.120 \mathrm{E}-04$ & 0.99974 \\
$\mathbf{3}$ & $\mathbf{3}$ & Green & 1.02565 & $-3.433 \mathrm{E}-03$ & 0.99973 \\
$\mathbf{4}$ & $\mathbf{4}$ & Red & 1.00034 & $-3.760 \mathrm{E}-03$ & 0.99941 \\
\hline $\mathbf{8 A}$ & 5 & $\begin{array}{c}\text { NIR } \\
\text { Estrecha }\end{array}$ & 1.00989 & $-2.867 \mathrm{E}-03$ & 0.99942 \\
\hline $\mathbf{8}$ & 5 & NIR Ancha & 1.01025 & $1.909 \mathrm{E}-02$ & 0.99381 \\
$\mathbf{1 1}$ & 6 & SWIR 1 & 0.99641 & $-3.151 \mathrm{E}-03$ & 0.99973 \\
\hline $\mathbf{1 2}$ & 7 & SWIR 2 & 0.98597 & $2.116 \mathrm{E}-04$ & 0.99972 \\
\hline
\end{tabular}



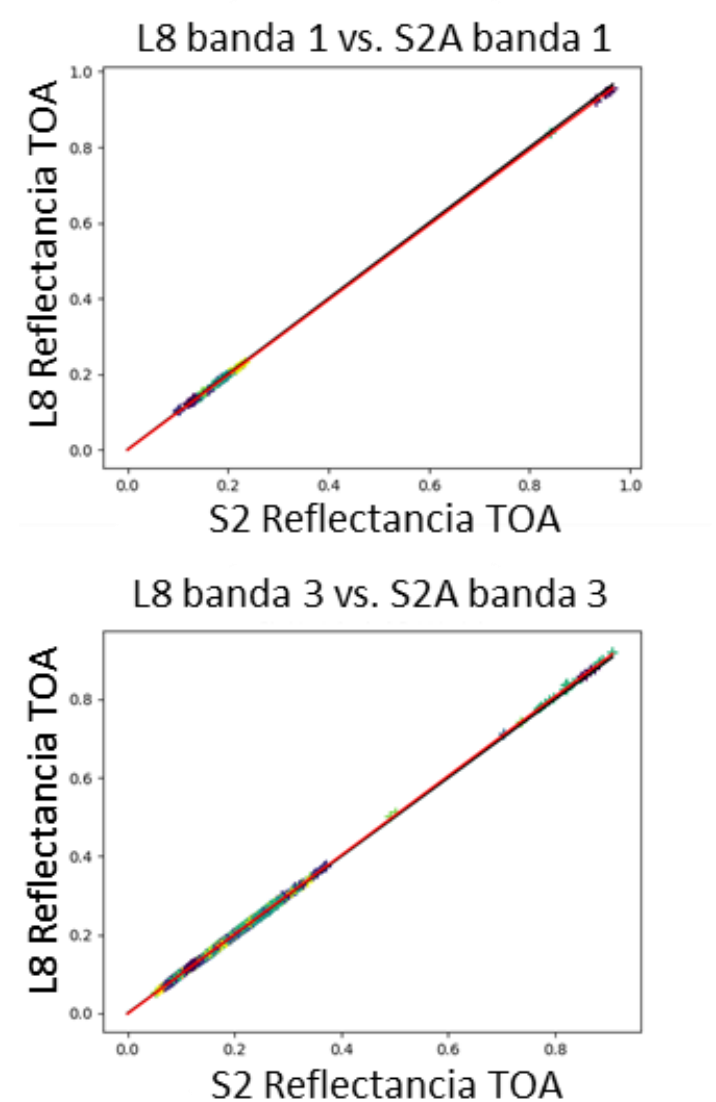

L8 banda 5 vs. S2A banda 8

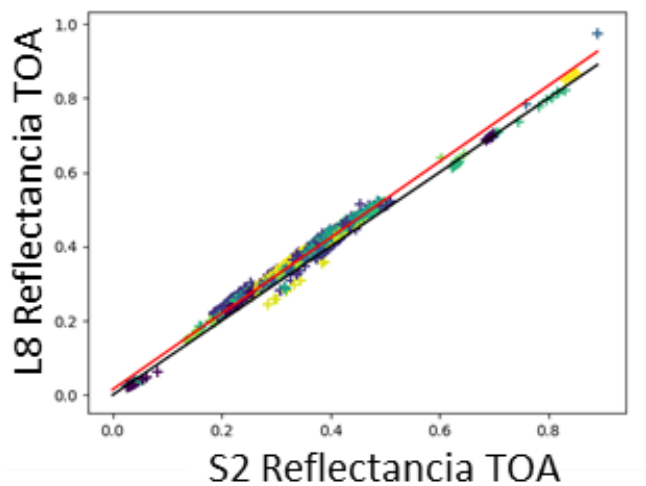

L8 banda 6 vs. S2A banda 11

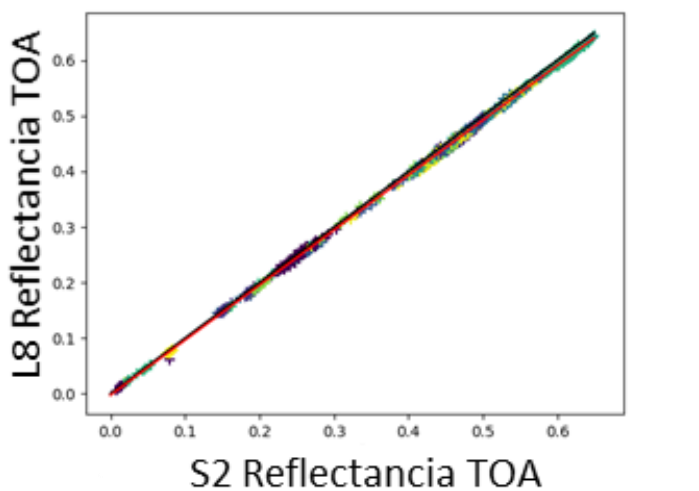

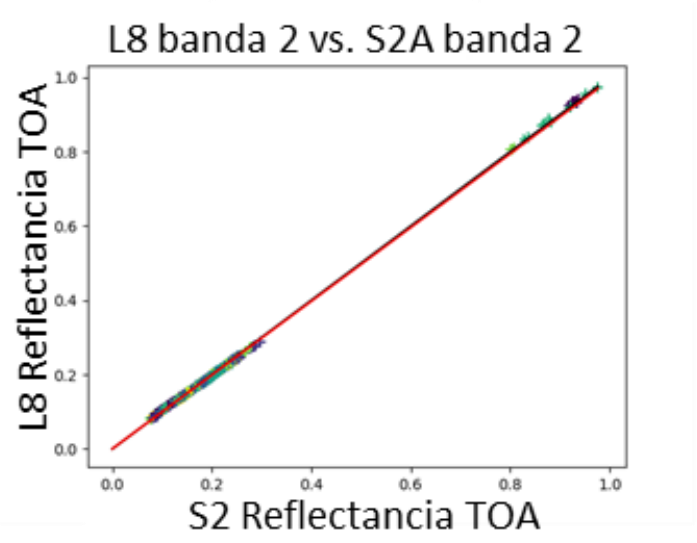

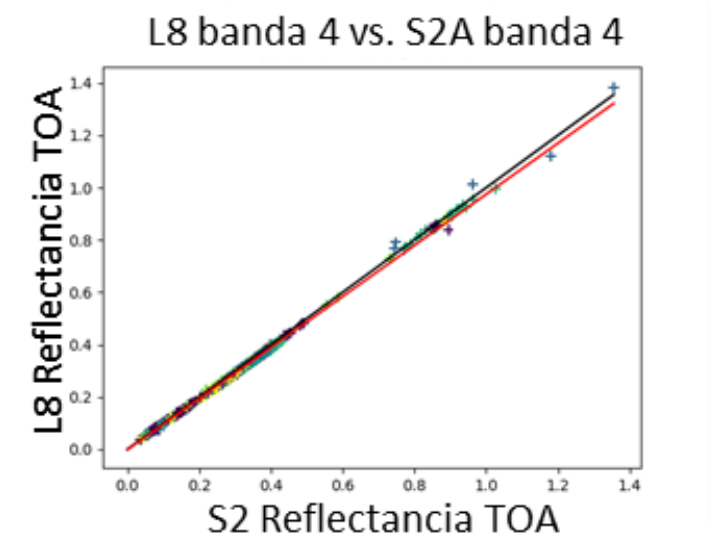

L8 banda 5 vs. S2A banda 8 A

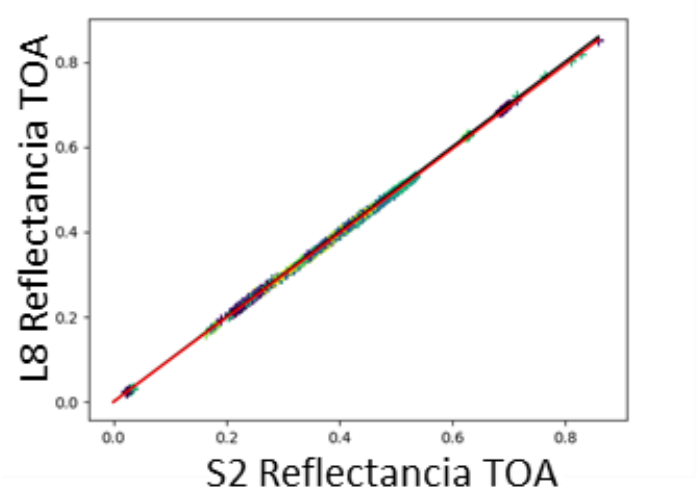

L8 banda 7 vs. S2A banda 12

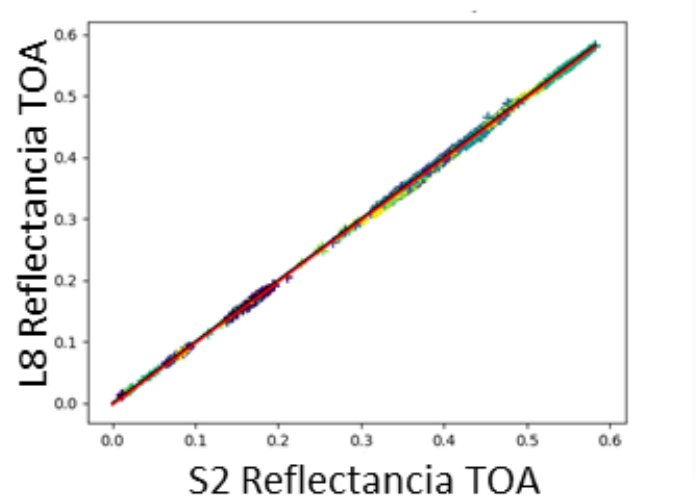

Figura 33. Gráficas de la regresión lineal para cada par de bandas a estudio de L8 y S2A, de los valores de reflectancia TOA calculados sobre zonas homogéneas. 

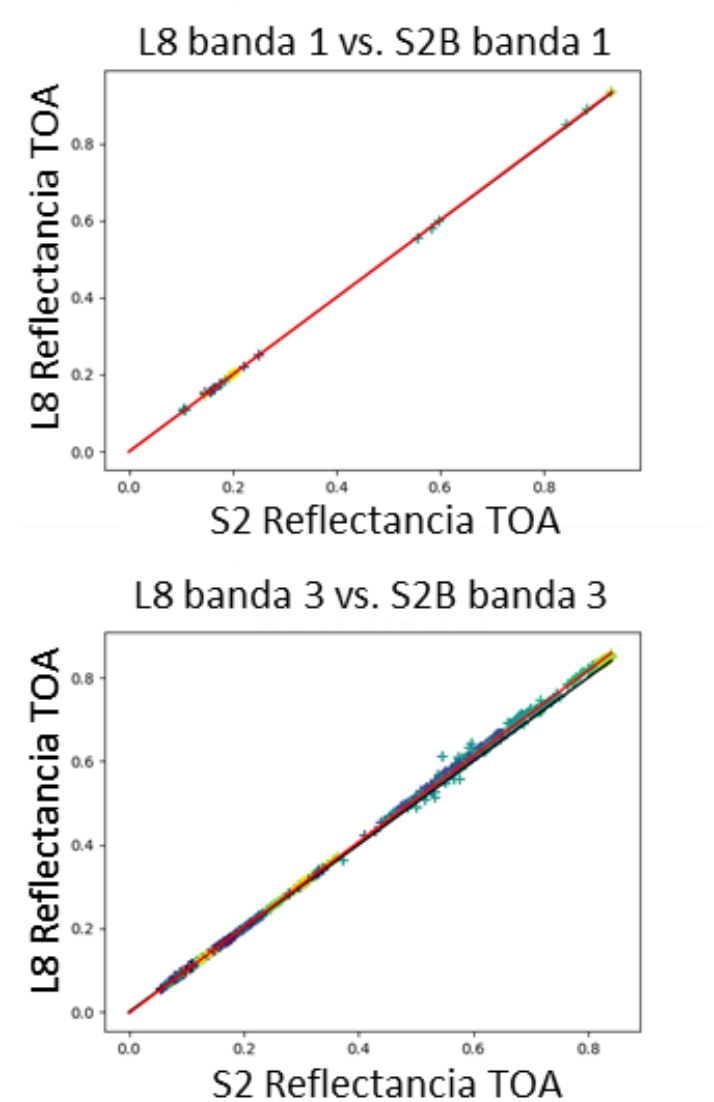

L8 banda 5 vs. S2B banda 8
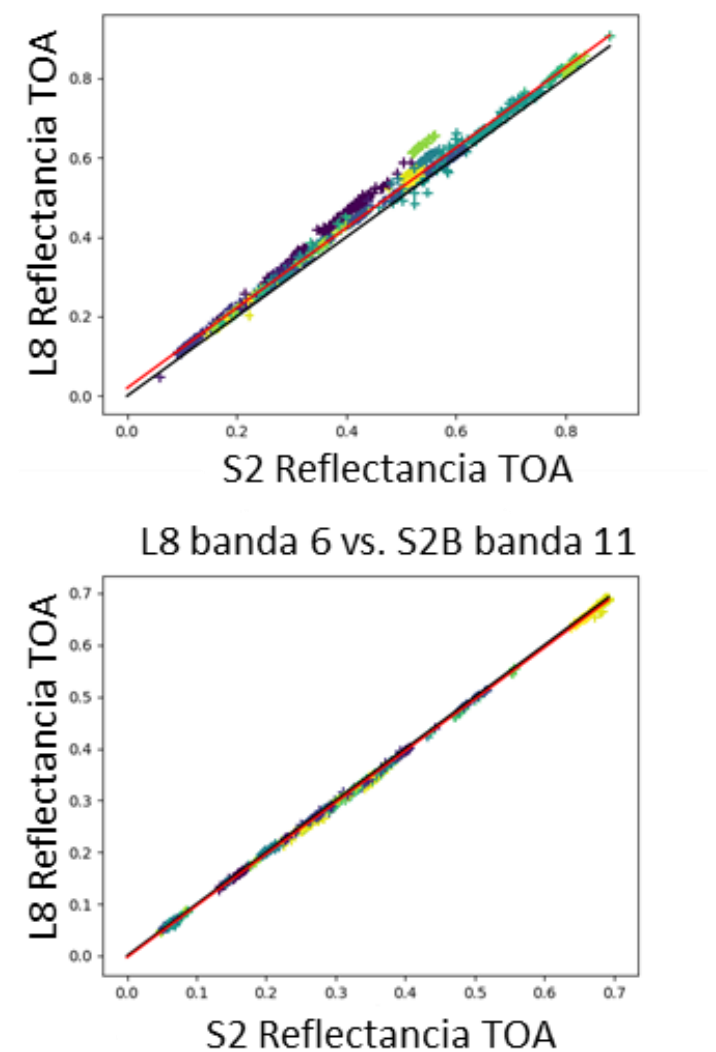
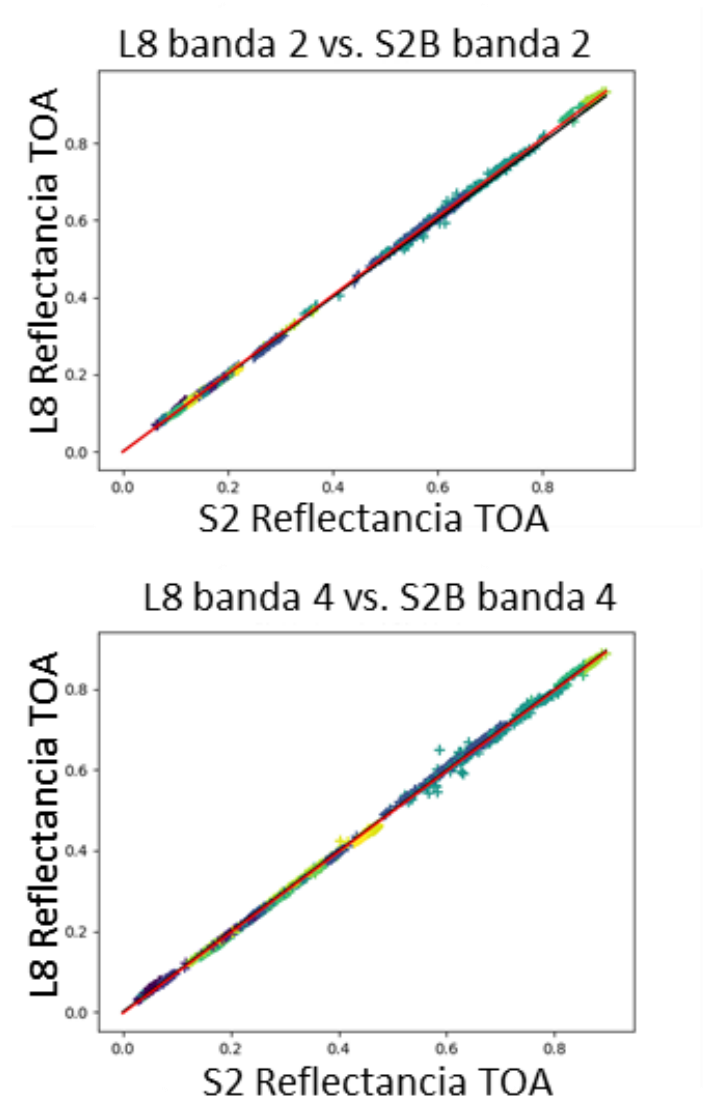

L8 banda 5 vs. S2B banda $8 \mathrm{~A}$
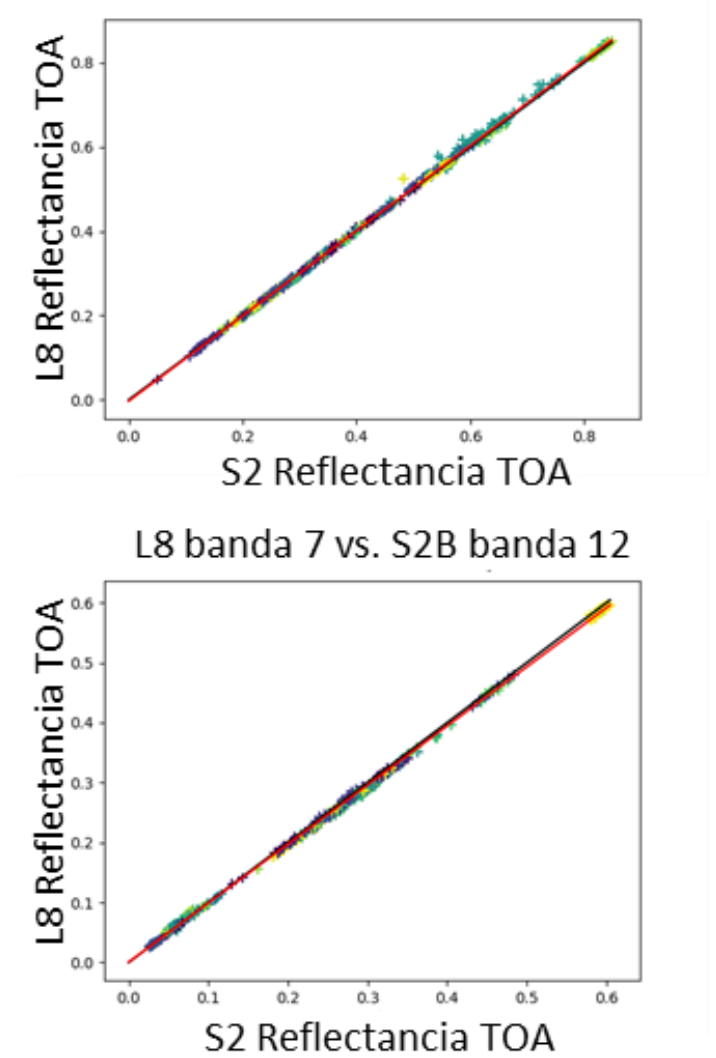

Figura 34. Gráficas de la regresión lineal para cada par de bandas a estudio de L8 y S2B, de los valores de reflectancia TOA calculados sobre zonas homogéneas. 
Este proceso de regresión lineal se volvió a repetir, pero eliminando el grado de libertad del offset $o$ intersección forzándolo a cero, debido a los resultados obtenidos y a que se puede asumir que la reflectancia TOA de los sensores en ausencia de señal será 0 . En la Tabla 5 y Tabla 6, así como en la Figura 35 y Figura 36, se presentan los resultados de esta nueva regresión lineal forzando a cero el valor de intersección. Cada punto es un área homogénea diferente, cada color hace referencia a la SNO correspondiente y la línea roja es la regresión lineal, mientras la línea negra es la pendiente con valor 1.

Tabla 5. Resultados de la regresión lineal con el valor de intersección forzado a cero de la comparativa de valores de reflectancia TOA para cada par de bandas de L8 y S2A.

L8 vs. S2A

\begin{tabular}{|c|c|c|c|c|}
\hline Banda S2 & Banda L8 & $\begin{array}{c}\text { Nombre de la } \\
\text { Banda S2 }\end{array}$ & Pendiente & $\mathbf{R}^{2}$ \\
\hline 1 & 1 & $\begin{array}{l}\text { Aerosol } \\
\text { Costero }\end{array}$ & 0.98911 & 0.99985 \\
\hline 2 & 2 & Blue & 0.99402 & 0.99814 \\
\hline 3 & 3 & Green & 1.00540 & 0.99931 \\
\hline 4 & 4 & Red & 0.97124 & 0.99741 \\
\hline $8 \mathrm{~A}$ & 5 & NIR Estrecha & 0.98999 & 0.99907 \\
\hline 8 & 5 & NIR Ancha & 1.06036 & 0.97962 \\
\hline 11 & 6 & SWIR 1 & 0.98441 & 0.99906 \\
\hline 12 & 7 & SWIR 2 & 0.99299 & 0.99914 \\
\hline
\end{tabular}

Tabla 6. Resultados de la regresión lineal con el valor de intersección forzado a cero, de la comparativa de valores de reflectancia TOA para cada par de bandas de L8 y S2B.

L8 vs. S2B

\begin{tabular}{|ccccc|}
\hline Banda S2 & Banda L8 & $\begin{array}{c}\text { Nombre de la } \\
\text { Banda S2 }\end{array}$ & Pendiente & $\mathbf{R}^{\mathbf{2}}$ \\
\hline $\mathbf{1}$ & 1 & $\begin{array}{c}\text { Aerosol } \\
\text { Costero }\end{array}$ & 1.00188 & 0.99980 \\
\hline $\mathbf{2}$ & $\mathbf{2}$ & Blue & 1.01277 & 0.99948 \\
\hline $\mathbf{3}$ & 3 & Green & 1.01872 & 0.99932 \\
\hline $\mathbf{4}$ & 4 & Red & 0.99326 & 0.99867 \\
$\mathbf{8 A}$ & 5 & NIR Estrecha & 1.00428 & 0.99877 \\
$\mathbf{8}$ & 5 & NIR Ancha & 1.04793 & 0.98434 \\
\hline $\mathbf{1 1}$ & 6 & SWIR 1 & 0.98985 & 0.99935 \\
\hline $\mathbf{1 2}$ & 7 & SWIR 2 & 0.98645 & 0.99943 \\
\hline
\end{tabular}



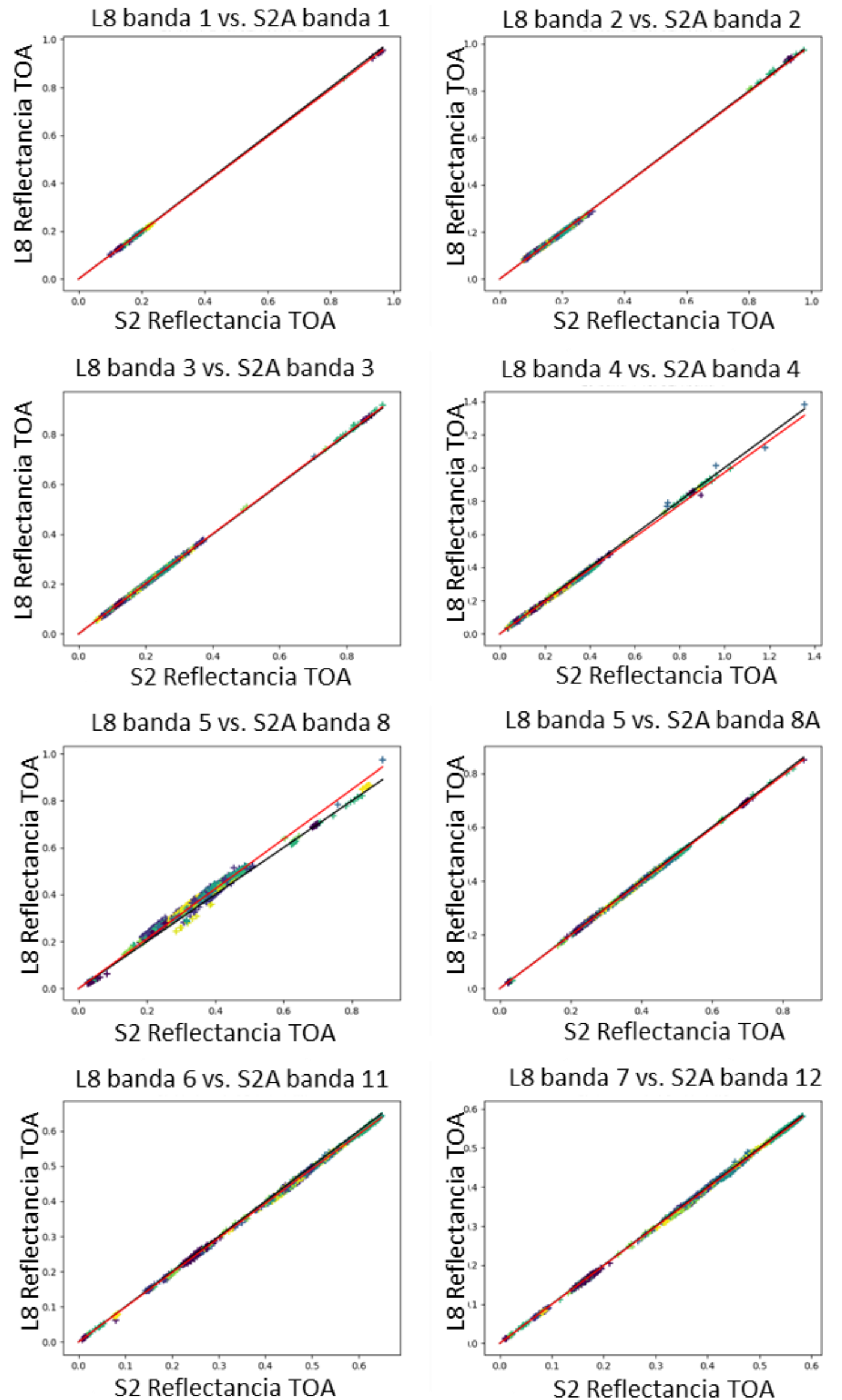

Figura 35. Regresión lineal de L8 vs. S2A con el valor de la intersección forzada a 0. 

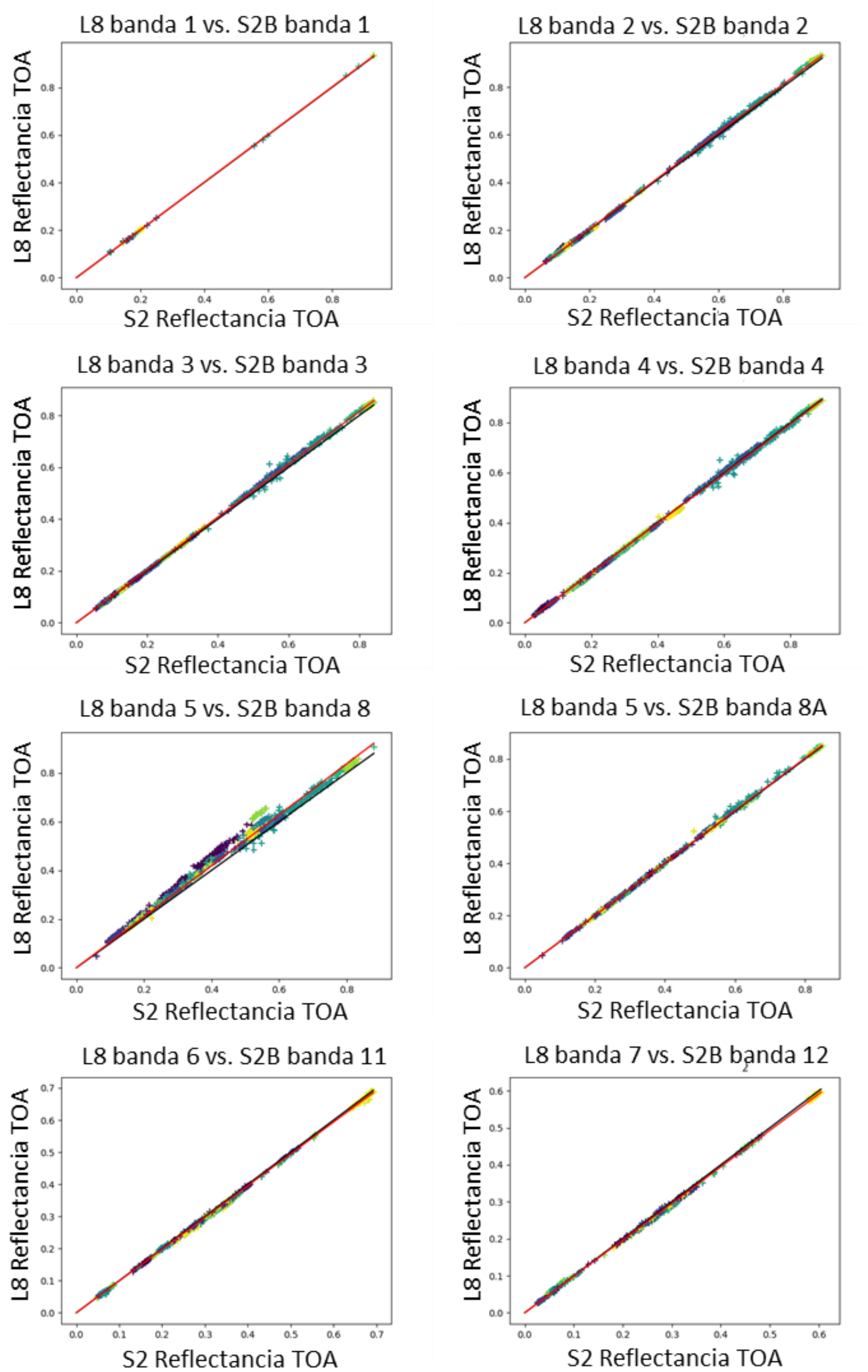

Figura 36. Regresión lineal de L8 vs. S2B con el valor de la intersección forzada a 0. 
Los resultados obtenidos en la Tabla 5 y Tabla 6 muestran un elevado valor de $\mathrm{R}^{2}$ con valores superiores a 0.99 para todas las bandas, con la excepción de la banda NIR ancha que presenta valores ligeramente inferiores en el rango de 0.97 - 0.98.

\subsubsection{Influencia de otras variables.}

Se analizaron diferentes variables que pudieran influir en la metodología, como fueron la reflectancia media, la $\sigma$ de la reflectancia, el ángulo de elevación y azimut solar en el centroide, la distancia de las áreas homogéneas de centroide al nadir, así como el valor de la latitud de las áreas homogéneas. Para ello se realizó un ajuste lineal de los agregados de los residuos para las SNOs completas, con la fecha de adquisición y los intervalos de tiempo entre adquisiciones. En ningún caso se detectó una relación entre los residuos y las variables anteriormente mencionadas.

En la Figura 37 se muestra un ejemplo del análisis de los diferentes valores de los residuos confrontados con la distancia al nadir, la elevación del sol, la fecha de adquisición y los intervalos de tiempo entre adquisiciones. Cada punto representa un área homogénea diferente, siendo los colores la representación de la SNOs a la que pertenecen.
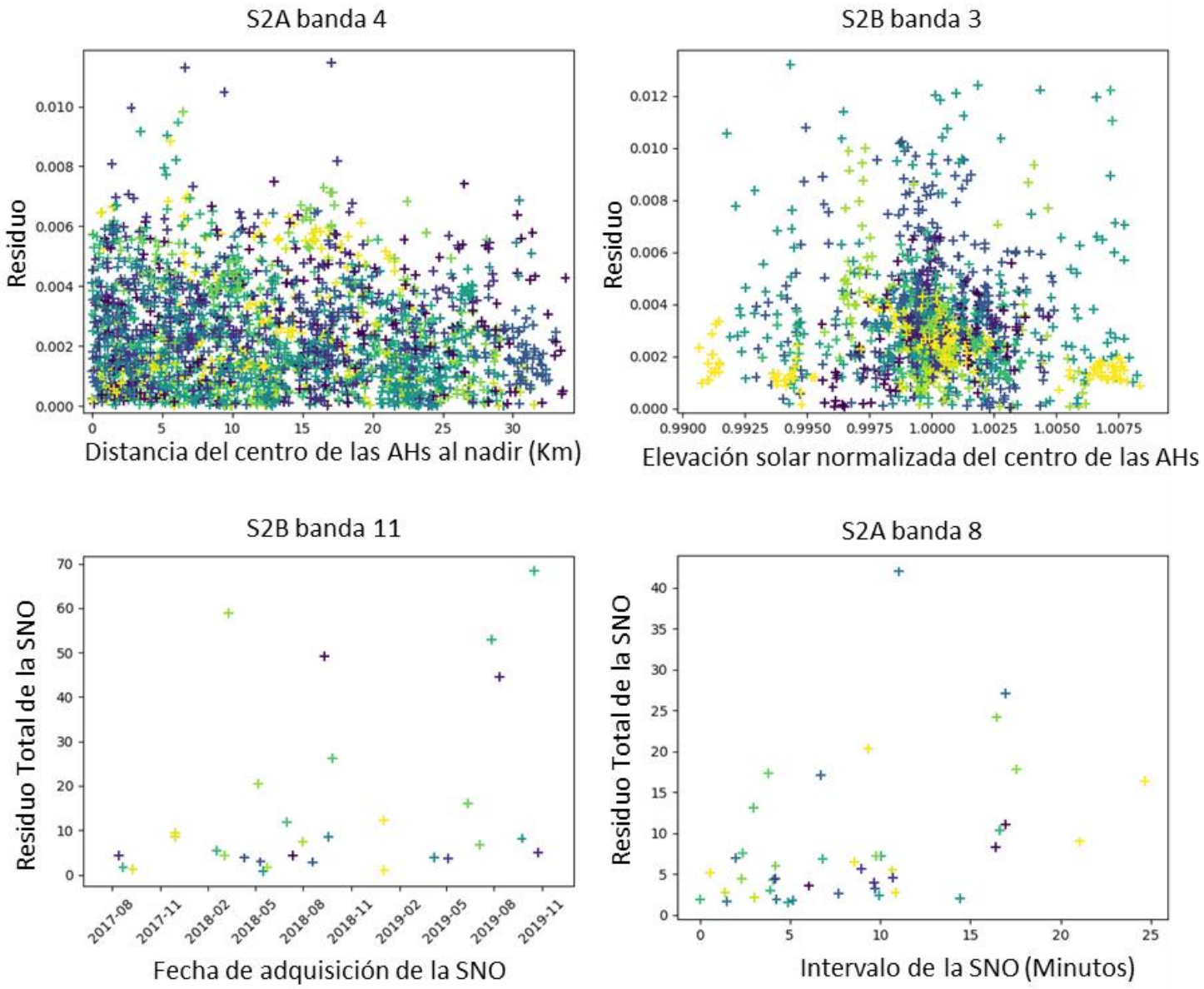

Figura 37. Regresión lineal de $L 8$ vs. S2B con el valor de la intersección forzada a 0. 


\subsubsection{Análisis de las clases de superfices utilizadas.}

Debido a la naturaleza global de la metodología y la distribución de las SNOs por toda la superficie de la tierra, se consideró conveniente el análisis de las diferentes clases de superficies implicadas. Esta información permite conocer la distribución y posible ponderación de cada clase de superficie. Para ello se utilizó el land cover CGLS-LC100 (Buchhorn et al., 2019) y el vector de áreas homogéneas de cada par de bandas y sensores S2. En la Tabla 7 se muestran los diferentes tipos de clases del CGLS-LC100, el identificador asignado, así como la paleta de colores personalizada para este trabajo. En la Figura 38 se muestra la distribución de las clases del CGLS-LC100 sobre las áreas homogéneas para cada banda de los sensores S2 A/B.

Tabla 7. Leyenda de las 23 clases del land cover CGLS-LC100. Fuente: Adaptado de Buchhorn et al., (2019).

\begin{tabular}{|c|c|c|}
\hline Color & ID & Nombre de la clase \\
\hline & 22 & Oceans, seas. Can be either fresh or salt-water bodies. \\
\hline & 21 & Open forest, not matching any of the other definitions. \\
\hline & 20 & Open forest, mixed. \\
\hline & 19 & $\begin{array}{l}\text { Open forest, deciduous broad leaf. Top layer- trees } 15-70 \% \text { and second layer- mixed of shrubs and } \\
\text { grassland, consists of seasonal broadleaf tree communities with an annual cycle of leaf-on and leaf- } \\
\text { off periods. }\end{array}$ \\
\hline & 18 & $\begin{array}{l}\text { Open forest, deciduous needle leaf. Top layer- trees } 15-70 \% \text { and second layer- mixed of shrubs and } \\
\text { grassland, consists of seasonal needle leaf tree communities with an annual cycle of leaf-on and } \\
\text { leaf-off periods. }\end{array}$ \\
\hline & 17 & $\begin{array}{l}\text { Open forest, evergreen broad leaf. Top layer- trees } 15-70 \% \text { and second layer- mixed of shrubs and } \\
\text { grassland, almost all broadleaf trees remain green year round. Canopy is never without green } \\
\text { foliage. }\end{array}$ \\
\hline & 16 & $\begin{array}{l}\text { Open forest, evergreen needle leaf. Top layer- trees } 15-70 \% \text { and second layer- mixed of shrubs and } \\
\text { grassland, almost all needle leaf trees remain green all year. Canopy is never without green foliage. }\end{array}$ \\
\hline & 15 & Closed forest, not matching any of the other definitions. \\
\hline & 14 & Closed forest, mixed. \\
\hline & 13 & $\begin{array}{l}\text { Closed forest, deciduous broad leaf. Tree canopy }>70 \% \text {, consists of seasonal broadleaf tree } \\
\text { communities with an annual cycle of leaf-on and leaf-off periods. }\end{array}$ \\
\hline & 12 & $\begin{array}{l}\text { Closed forest, deciduous needle leaf. Tree canopy }>70 \% \text {, consists of seasonal needle leaf tree } \\
\text { communities with an annual cycle of leaf-on and leaf-off periods. }\end{array}$ \\
\hline & 11 & $\begin{array}{l}\text { Closed forest, evergreen broad leaf. Tree canopy }>70 \% \text {, almost all broadleaf trees remain green } \\
\text { year round. Canopy is never without green foliage. }\end{array}$ \\
\hline & 10 & $\begin{array}{l}\text { Closed forest, evergreen needle leaf. Tree canopy }>70 \% \text {, almost all needle leaf trees remain green } \\
\text { all year. Canopy is never without green foliage. }\end{array}$ \\
\hline & 9 & Moss and lichen. \\
\hline & 8 & $\begin{array}{l}\text { Herbaceous wetland. Lands with a permanent mixture of water and herbaceous or woody } \\
\text { vegetation. The vegetation can be present in either salt, brackish, or fresh water. }\end{array}$ \\
\hline & 7 & Permanent water bodies. Lakes, reservoirs, and rivers. Can be either fresh or salt-water bodies. \\
\hline & 6 & Snow and ice. Lands under snow or ice cover throughout the year. \\
\hline & 5 & $\begin{array}{l}\text { Bare / sparse vegetation. Lands with exposed soil, sand, or rocks and never has more than } 10 \% \\
\text { vegetated cover during any time of the year. }\end{array}$ \\
\hline & 4 & Urban / built up. Land covered by buildings and other man-made structures. \\
\hline & 3 & $\begin{array}{l}\text { Cultivated and managed vegetation / agriculture. Lands covered with temporary crops followed by } \\
\text { harvest and a bare soil period (e.g., single and multiple cropping systems). Note that perennial } \\
\text { woody crops will be classified as the appropriate forest or shrub land cover type. }\end{array}$ \\
\hline & 2 & $\begin{array}{l}\text { Herbaceous vegetation. Plants without persistent stem or shoots above ground and lacking definite } \\
\text { firm structure. Tree and shrub cover is less than } 10 \% \text {. }\end{array}$ \\
\hline & 1 & $\begin{array}{l}\text { Shrubs. Woody perennial plants with persistent and woody stems and without any defined main } \\
\text { stem being less than } 5 \mathrm{~m} \text { tall. The shrub foliage can be either evergreen or deciduous. }\end{array}$ \\
\hline & 0 & Unknown \\
\hline
\end{tabular}




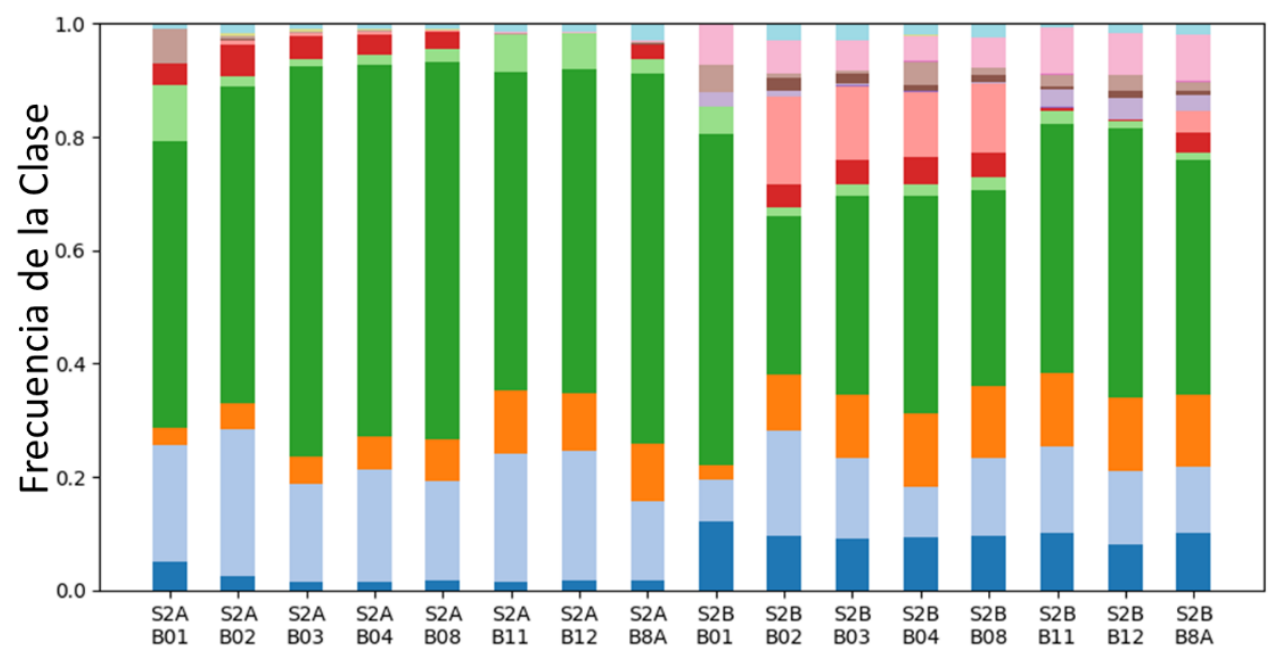

Figura 38. Distribución de clases del CGLS-LC100 sobre las áreas homogéneas de las bandas de S2A y $S 2 B$.

Para un conocimiento más detallado del comportamiento de la metodología de SNOs en las diferentes superficies localizadas en el CGLS-LC100, se realizó un análisis de las mismas. Para ello, mediante una regresión lineal se estudiaron las áreas homogéneas de forma individualizada con respecto a las clases más frecuentes. Esta comparativa se focalizó principalmente en las bandas más susceptibles de tener mayor incertidumbre debido al tipo de superficie utilizada (principalmente vegetación y tierras de cultivo), como son las bandas NIR y red. En la Tabla 8, Tabla 9 y Tabla 10, se muestran los resultados de las regresiones lineales de la banda red (4) y bandas NIR ( 8 y $8 \mathrm{~A}$ ) de S2A/B con respecto al par de bandas correspondiente en L8, utilizando las clases más frecuentes y sus correspondientes áreas homogéneas de forma individualizada.

Tabla 8. Resultados de la regresión lineal para las bandas 4 de S2 y L8 de las clases más frecuentes.

MSI banda 4, OLI banda 4 (red). Clases Más Frecuentes

\begin{tabular}{|c|c|c|c|c|c|}
\hline Satélite & Pendiente & $\mathbf{R}^{2}$ & $\begin{array}{l}\text { Número de } \\
\text { Áreas } \\
\text { Homogéneas }\end{array}$ & Clases & $\begin{array}{l}\text { Descripción de } \\
\text { las Clases }\end{array}$ \\
\hline S2A & 0.97124 & 0.99741 & 2376 & \multirow{2}{*}{ Todas } & \multirow{2}{*}{ Todas las clases } \\
\hline S2B & 0.99444 & 0.99873 & 1702 & & \\
\hline S2A & 0.96765 & 0.99310 & 1558 & \multirow[b]{2}{*}{5} & \multirow{2}{*}{$\begin{array}{l}\text { Suelo desnudo / } \\
\text { Vegetación } \\
\text { escasa }\end{array}$} \\
\hline S2B & 0.97898 & 0.99876 & 655 & & \\
\hline$S 2 A$ & 0.97106 & 0.99599 & 142 & \multirow[b]{2}{*}{3} & \multirow{2}{*}{$\begin{array}{l}\text { Vegetación } \\
\text { cultivada y } \\
\text { gestionada / } \\
\text { agrícola }\end{array}$} \\
\hline S2B & 0.98698 & 0.99378 & 221 & & \\
\hline$S 2 A$ & 0.97171 & 0.99511 & 471 & \multirow{2}{*}{2} & \multirow{2}{*}{$\begin{array}{l}\text { Vegetación } \\
\text { herbácea }\end{array}$} \\
\hline S2B & 0.99318 & 0.99932 & 152 & & \\
\hline S2A & 0.97181 & 0.99857 & 33 & \multirow{2}{*}{1} & \multirow{2}{*}{ Arbustos } \\
\hline S2B & 0.97378 & 0.99667 & 158 & & \\
\hline
\end{tabular}


Tabla 9. Resultados de la regresión lineal para la banda 8 de $\$ 2$ y la banda 5 de L8 de las clases más frecuentes

MSI banda 8, OLI banda 5 (NIR). Clases Más Frecuentes

\begin{tabular}{|c|c|c|c|c|c|}
\hline Satélite & Pendiente & $\mathbf{R}^{2}$ & $\begin{array}{l}\text { Número de } \\
\text { Áreas } \\
\text { Homogéneas }\end{array}$ & Clases & $\begin{array}{c}\text { Descripción de las } \\
\text { Clases }\end{array}$ \\
\hline S2A & 1.06036 & 0.97962 & 2116 & \multirow{2}{*}{ Todas } & \multirow{2}{*}{ Todas las clases } \\
\hline S2B & 1.04793 & 0.98434 & 1568 & & \\
\hline S2A & 1.05939 & 0.94730 & 1409 & \multirow{2}{*}{5} & \multirow{2}{*}{$\begin{array}{l}\text { Suelo desnudo / } \\
\text { Vegetación escasa }\end{array}$} \\
\hline S2B & 1.06221 & 0.99484 & 544 & & \\
\hline S2A & 1.09840 & 0.98633 & 159 & \multirow[b]{2}{*}{3} & \multirow{2}{*}{$\begin{array}{c}\text { Vegetación } \\
\text { cultivada y } \\
\text { gestionada / } \\
\text { agrícola }\end{array}$} \\
\hline S2B & 1.11746 & 0.99203 & 200 & & \\
\hline S2A & 1.09205 & 0.96258 & 369 & \multirow{2}{*}{2} & \multirow{2}{*}{$\begin{array}{l}\text { Vegetación } \\
\text { herbácea }\end{array}$} \\
\hline S2B & 1.05531 & 0.97312 & 214 & & \\
\hline S2A & 1.09564 & 0.97286 & 37 & \multirow[b]{2}{*}{ 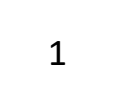 } & \multirow{2}{*}{ Arbustos } \\
\hline S2B & 1.07379 & 0.98090 & 151 & & \\
\hline
\end{tabular}

Tabla 10. Resultados de la regresión lineal para la banda $8 \mathrm{~A}$ de S2 y la banda 5 de L8 de las clases más frecuentes

MSI banda 8A, OLI banda 5 (NIR). Clases Más Frecuentes

\begin{tabular}{|c|c|c|c|c|c|}
\hline Satélite & Pendiente & $\mathbf{R}^{2}$ & $\begin{array}{l}\text { Número de } \\
\text { Áreas } \\
\text { Homogéneas }\end{array}$ & Clases & $\begin{array}{c}\text { Descripción de las } \\
\text { Clases }\end{array}$ \\
\hline S2A & 0.98999 & 0.99907 & 887 & \multirow{2}{*}{ Todas } & \multirow{2}{*}{ Todas las clases } \\
\hline S2B & 1.00428 & 0.99877 & 589 & & \\
\hline S2A & 0.98955 & 0.99589 & 583 & \multirow{2}{*}{5} & \multirow{2}{*}{$\begin{array}{l}\text { Suelo desnudo / } \\
\text { Vegetación escasa }\end{array}$} \\
\hline S2B & 1.00059 & 0.99909 & 257 & & \\
\hline S2A & 0.99376 & 0.99428 & 91 & \multirow[b]{2}{*}{3} & \multirow{2}{*}{$\begin{array}{c}\text { Vegetación } \\
\text { cultivada y } \\
\text { gestionada / } \\
\text { agrícola }\end{array}$} \\
\hline S2B & 1.00376 & 0.99904 & 75 & & \\
\hline S2A & 0.98838 & 0.99583 & 123 & \multirow{2}{*}{2} & \multirow{2}{*}{$\begin{array}{l}\text { Vegetación } \\
\text { herbácea }\end{array}$} \\
\hline S2B & 1.00338 & 0.99960 & 69 & & \\
\hline S2A & 0.99168 & 0.99641 & 16 & \multirow{2}{*}{1} & \multirow{2}{*}{ Arbustos } \\
\hline S2B & 0.99264 & 0.99614 & 61 & & \\
\hline
\end{tabular}




\subsection{Discusión}

Se ha presentado una metodología empírica de SNOs sobre áreas homogéneas para lo que no es necesario realizar ningún tipo de corrección atmosférica o de BRDF. A diferencia de la utilización de los PICS como target de cross-calibration, los cuales están perfectamente definidos, caracterizados y con una localización fija (Neigh et al., 2015; Mishra et al., 2014), la metodología de SNOs presentada tiene un ámbito de aplicación global, donde el área de estudio de cada SNO está caracterizada y definida en base a criterios orbitales de cruce de las trayectorias terrestres entre satélites, al establecimiento de un umbral de 30 minutos de intervalo de tiempo entre adquisiciones y la localización de áreas homogéneas donde realizar la comparativa entre sensores mediante un criterio de homogeneidad a partir del CV.

Los resultados obtenidos sobre una gran variedad de superficies espectrales (Figura 38) muestran una gran correlación entre los pares de bandas a estudio de S2A-MSI y S2BMSI con respecto a L8-OLI (Tabla 5 y Tabla 6). El uso de una gran variedad de superficies en la comparativa (Figura 38) permite cubrir prácticamente todo el rango de reflectancias TOA en la misma (Figura 35 y Figura 36), mientras que en otro tipo de metodologías como es el caso de los PICS, este rango está muy limitado a un rango elevado de reflectancias (Chander et al., 2013; Chander et al., 2010). A pesar de la diversidad de superficies utilizadas, el $\mathrm{R}^{2}$ muestra valores superiores a 0.997 en el caso de S2A y 0.999 en S2B, para todas las bandas a excepción de la banda NIR ancha (banda 8 de S2). La mayor incertidumbre en los resultados se registra en los pares de bandas con diferente RSRs.

Para la validación del método, fue necesario realizar la comparativa con diferentes metodologías y targets. Siguiendo las recomendaciones de Helder et al. (2018) para la validación de los métodos de los sensores MSI y OLI, se utilizó la métrica del ratio como comparativa entre metodologías, al eliminar de la misma posibles errores sistemáticos dentro de los modelos y proporcionar una comparación por modelo.

Helder et al. (2018) aplican en su comparativa entre los sensores S2A-MSI y L8-OLI, dos modelos diferentes de PICS que utilizan datos hiperespectrales de EO-1 Hyperion para el caso de la metodología The South Dakota State University Absolute PICS (APICS) (Mishra et al., 2014), y datos Envisat-MERIS para el método DIMITRI-PICS (Bouvet, 2014). También se añaden a la comparativa dos metodologías de calibración vicaria realizadas por la University of Arizona en Railroad Valley Playa, Nevada (RadCaTS RRV) y por South Dakota State University en Brookings vegetated (Brookings Vegetated site) (Anderson et al., 2013; Thome et al., 2004). En ambas metodologías vicarias se utilizó el software MODTRAN para predecir la reflectancia TOA a partir de las mediciones de superficie en los targets de calibración (Helder et al., 2018). En la Figura 39 se muestra la comparativa de los ratios de las pendientes de S2A-MSI y L8-OLI, para los 4 métodos anteriormente 
mencionados junto con los resultados obtenidos mediante la metodología de las SNOs a partir de áreas homogéneas (Tabla 5).

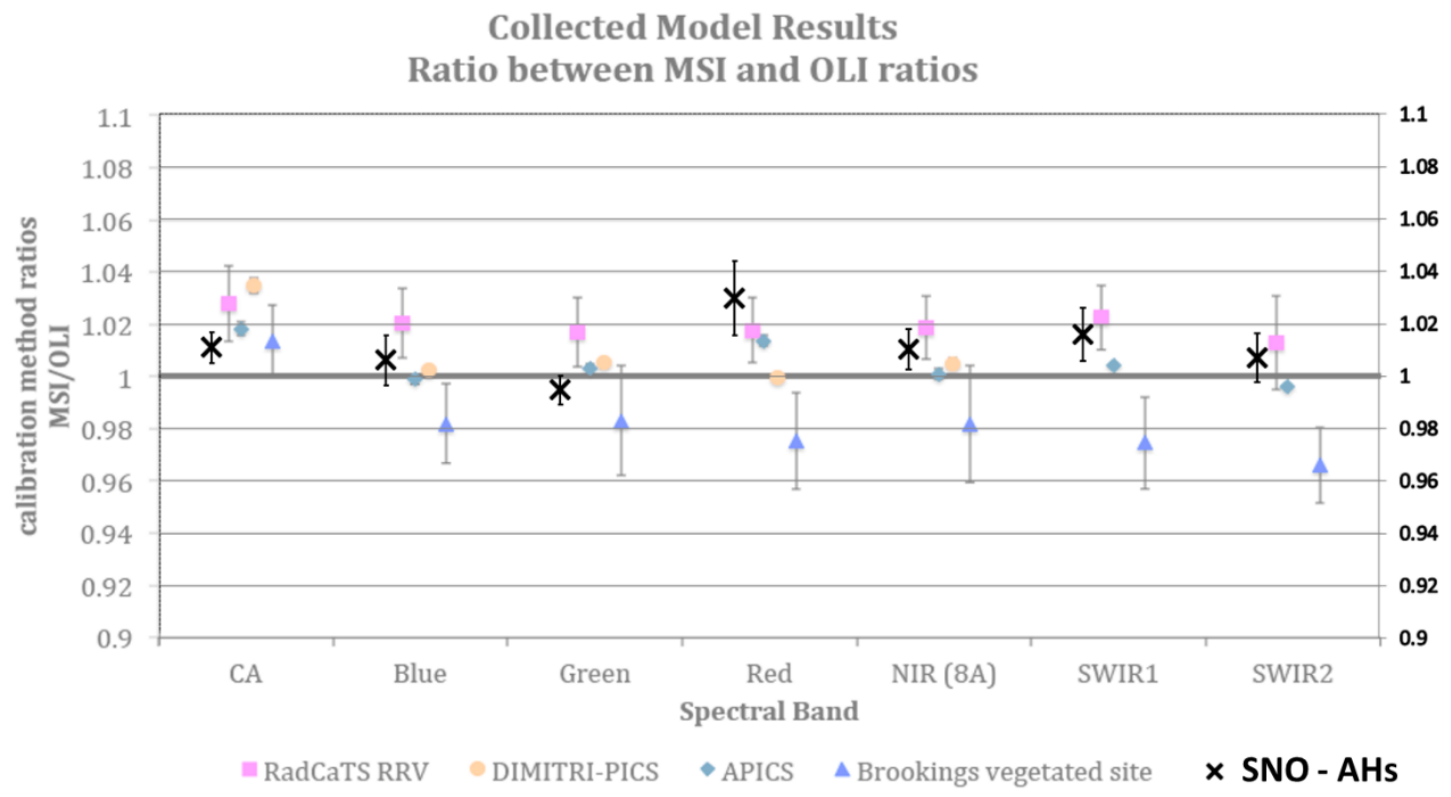

Figura 39. Comparativa de los ratios de las pendientes de S2A-MSI y L8-OLI para los métodos de Helder et al., (2018) y la metodología de SNOs mediante áreas homogéneas, representada por cruces negras sólidas en la gráfica. La metodología de SNOs muestra en sus resultados un intervalo de confianza de $\pm 3 \sigma$.

The Copernicus Sentinel-2 Mission Performance Centre (MPC) realiza de forma periódica un informe sobre el estado de la calibración de la misión S2 (ESA, 2020c). En el mismo, se hace la comparativa entre diferentes metodologías siguiendo la métrica de Helder et al. (2018). MPC realiza también el análisis de resultados a partir del ratio de las pendientes utilizando las metodologías de retrodispersión atmosférica de Rayleigh sobre zonas oceánicas, datos in situ, PICS caracterizados espectralmente y una comparativa de los sensores S2 con L8 y entre ellos mismos sobre Libya-4 (ESA, 2020c). En la Figura 40 y Figura 41 se muestran los resultados y la comparativa entre los métodos evaluados por MPC y la metodología de SNOs. 


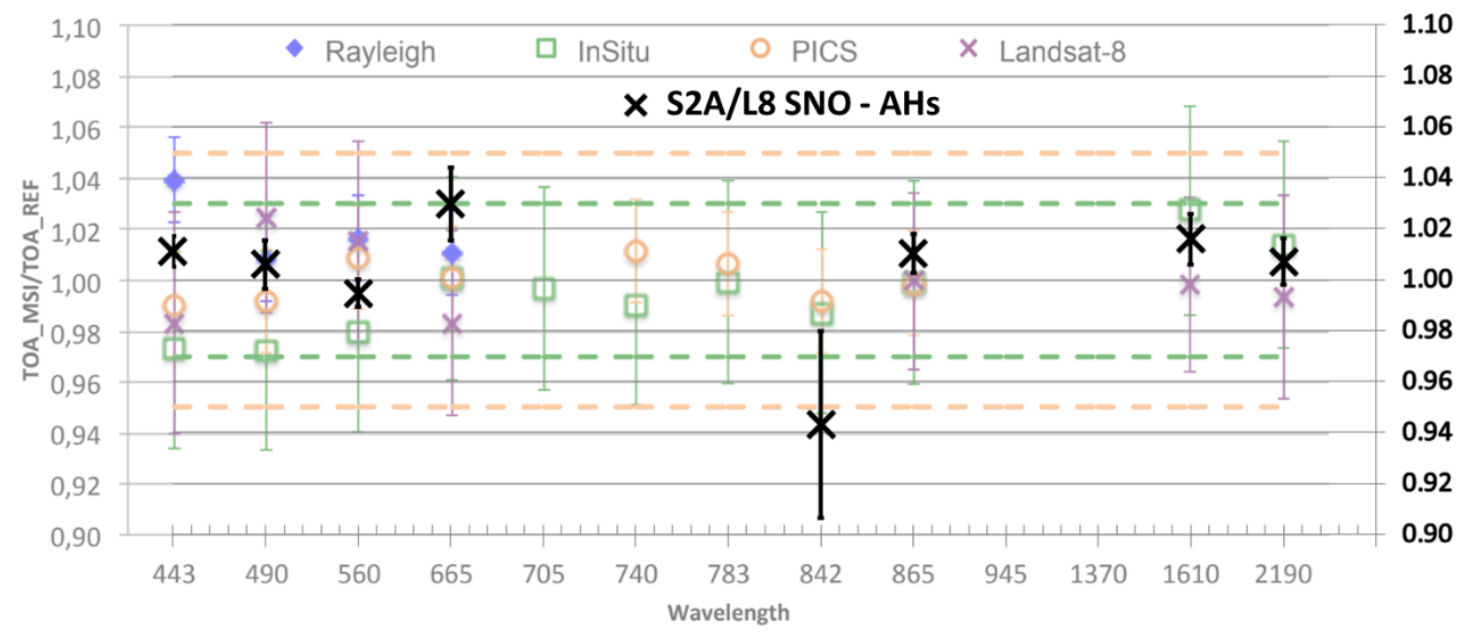

Figura 40. Comparativa de los ratios de las pendientes de S2A-MSI y L8-OLI para los métodos utilizados por el MPC y la metodología de SNOs mediante áreas homogéneas, representada por cruces negras sólidas en la gráfica. La metodología de SNOs muestra en sus resultados un intervalo de confianza de $\pm 3 \sigma$.

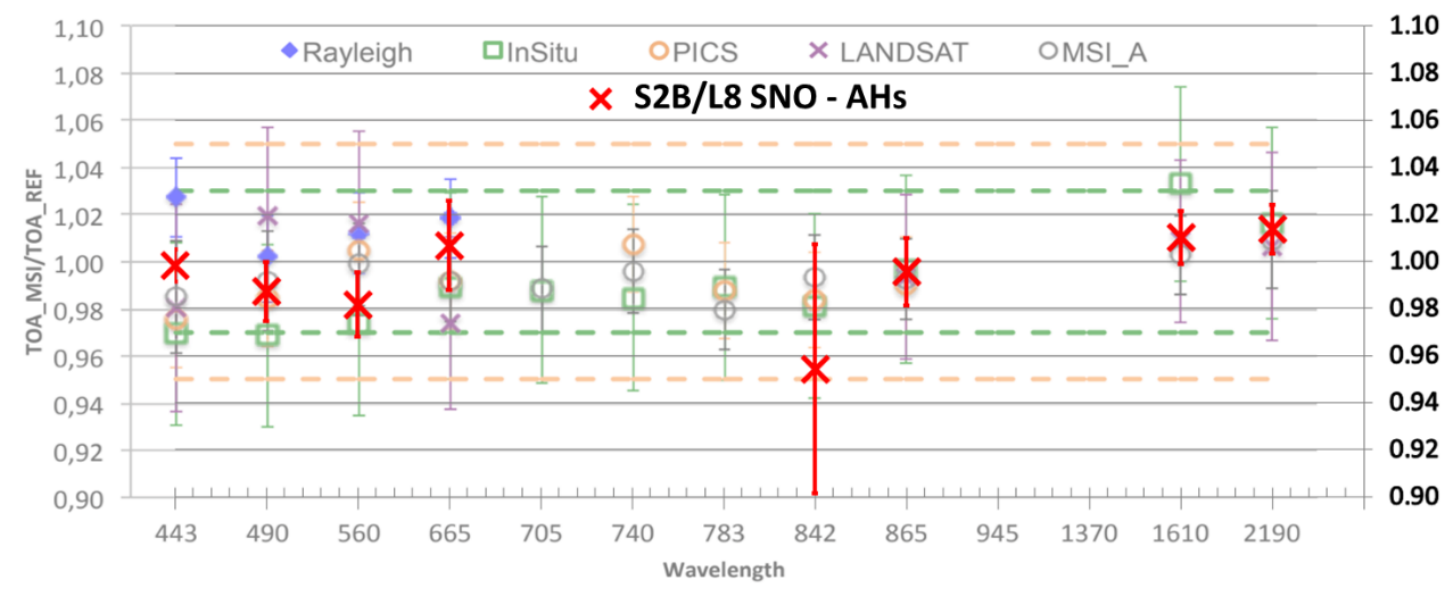

Figura 41. Comparativa de los ratios de las pendientes de S2B-MSI y L8-OLI para los métodos utilizados por el MPC y la metodología de SNOs mediante áreas homogéneas, representada por cruces rojas sólidas en la gráfica. La metodología de SNOs muestra en sus resultados un intervalo de confianza de $\pm 3 \sigma$.

Barsi et al., (2018) desarrollaron una metodología de cross-calibration de S2 A/B y L8 mediante SNOs en diferentes PICS aplicando un Spectral Band Adjustment Factor (SBAF) para mitigar las diferencias de las RSRs de los sensores. A partir de la citada publicación, el número de SNOs sobre los PICS utilizados (Algeria-3, Algeria-5, Egypt-1 y Libya-4) se ha incrementado, por lo que mostramos los últimos resultados actualizados en el $3^{\text {rd }}$ Sentinel-2 Validation Team Meeting (ESA, 2020d). En la Figura 42 y Figura 43 se muestra la comparativa de los resultados de Barsi et al., (ESA, 2020d) con la metodología de SNOs a partir de zonas homogéneas. 


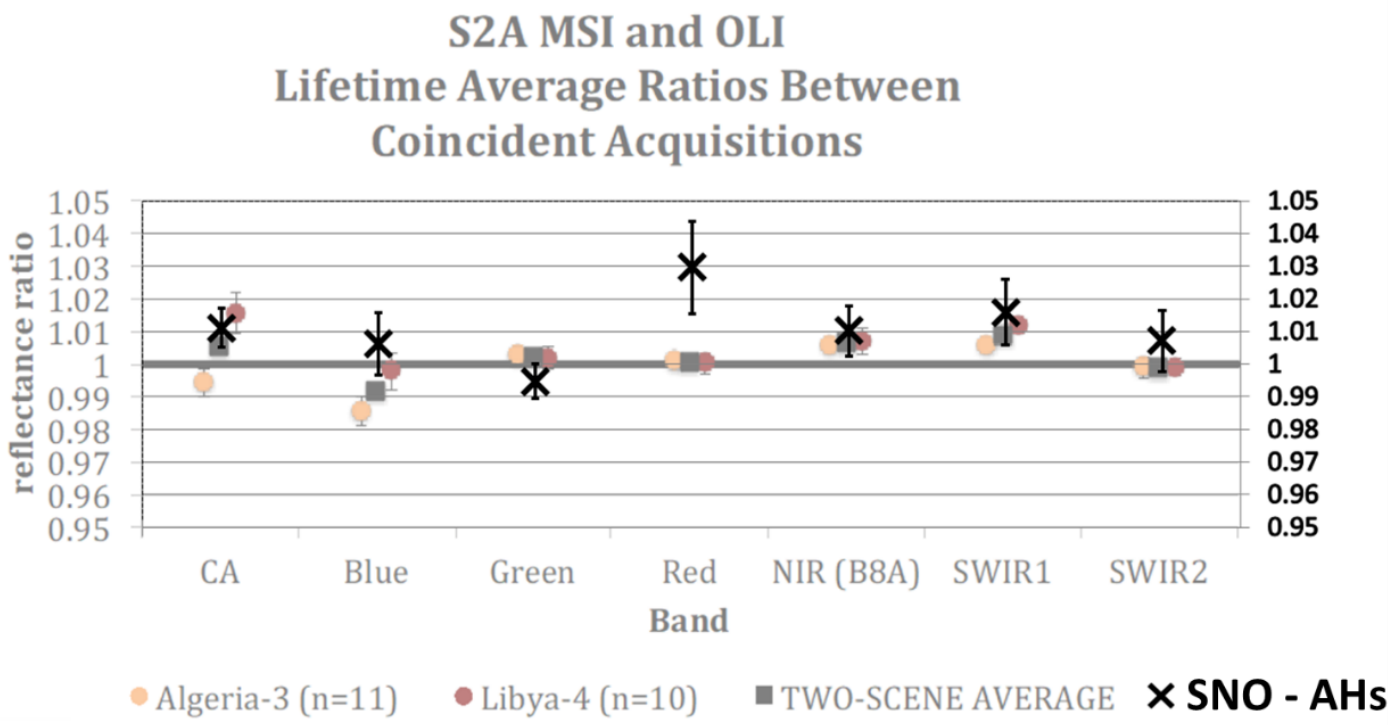

Figura 42. Comparativa de los ratios de las pendientes de S2A-MSI y L8-OLI para la metodología de Barsi et al., (2018) y la metodología de SNOs mediante áreas homogéneas, representada por cruces negras sólidas en la gráfica. La metodología de SNOs muestra en sus resultados un intervalo de confianza de $\pm 3 \sigma$.

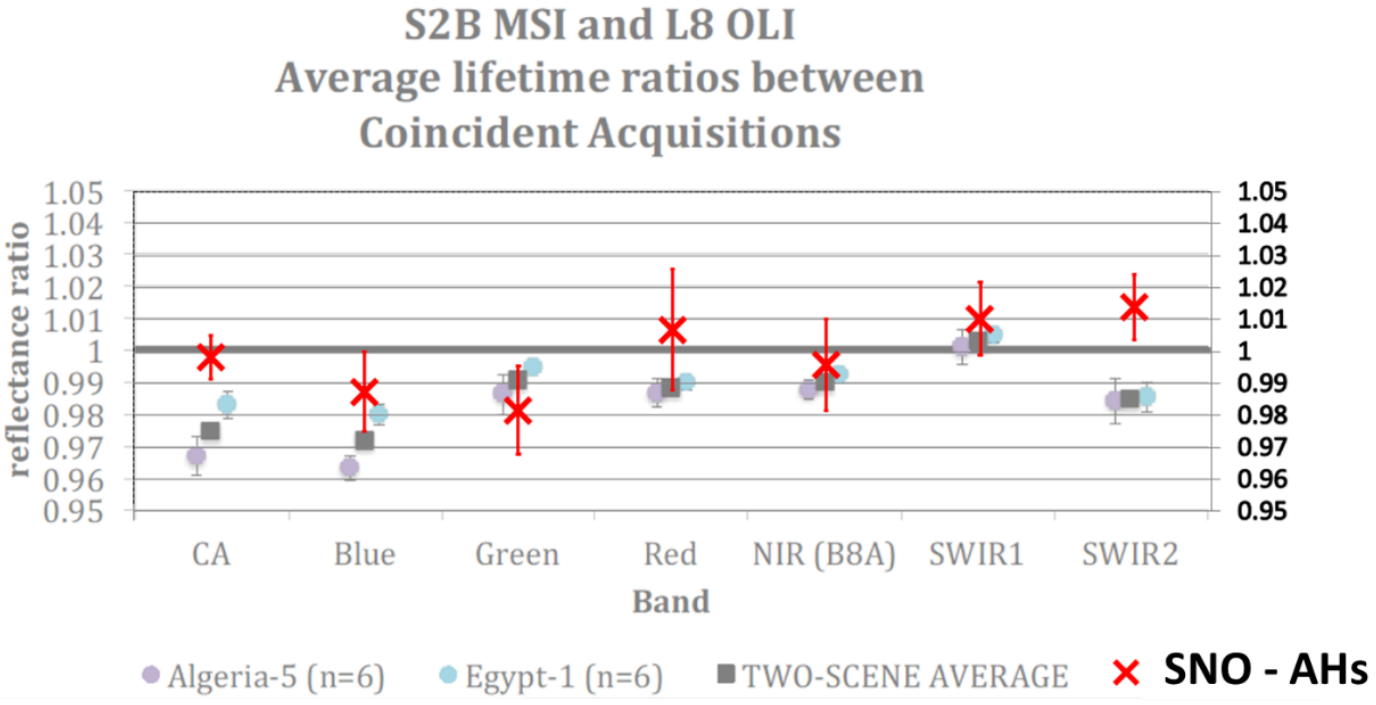

Figura 43. Comparativa de los ratios de las pendientes de S2B-MSI y L8-OLI para la metodología de Barsi et al., (2018) y la metodología de SNOs mediante áreas homogéneas, representada por cruces rojas sólidas en la gráfica. La metodología de SNOs muestra en sus resultados un intervalo de confianza de $\pm 3 \sigma$.

De forma general, los resultados de la comparativa de S2A con la metodología de SNOs muestran valores de reflectancia TOA más elevados que los obtenidos por S2B. Es importante resaltar que incluso en el análisis individual de las clases de superficies más frecuentes, también se repite esta situación entre S2A y S2B (Tabla 8, Tabla 9 y Tabla 
10). Estas diferencias son del orden del 1-2\%, siendo detectada por el MPC y encontrándose actualmente bajo estudio por este organismo (ESA, 2020c). Tanto en la comparativa con otras metodologías (Figura 40, Figura 41, Figura 42 y Figura 43) como en la comparativa de los resultados de la metodología de SNOs entre L8 vs S2A y L8 vS S2B (Tabla 5 y Tabla 6) este comportamiento se confirma.

Se observa también una mayor incertidumbre en los resultados de los pares de bandas con una mayor diferencia en su RSRs. Un ejemplo claro de esta situación es la banda 8 NIR ancha de S2, donde los valores de $R^{2}$ en la metodología de SNOs con respecto a la banda NIR de $L 8$ son los más bajos, con valores de 0.980 y 0.984 para S2 A/B, respectivamente. Este impacto de la RSRs también se ve reflejado de manera gráfica en la comparativa con otras metodologías, en los valores e intervalos de confianza de los mismos especialmente para la banda 8 NIR de S2, y en menor medida para las bandas red y green, al ser los pares de bandas entre S2 A/B y L8 con mayor diferencia en sus RSRs (Figura 21).

Los altos valores de $\mathrm{R}^{2}$ obtenidos para todas las bandas a estudio, con la salvedad de la banda 8 NIR ancha de S2 con unos valores menores del índice, junto con los excelentes resultados de la metodología de SNOs con otros métodos, validan y confirman este nuevo enfoque propuesto para la interoperabilidad y armonización de los datos de sensores de satélite.

\subsection{Conclusiones}

En este capítulo, se ha expuesto una metodología global de cross-calibration basada en SNOs a partir de áreas homogéneas sobre cualquier tipo de superficie. Esta metodología con fuerte fundamento empírico, presenta ventajas muy importantes con respecto a otros métodos, como por ejemplo los PICS. Para la metodología de SNOs no es necesaria la aplicación de modelos de BRDF, ni de corrección atmosférica, además de obtener un amplio rango de reflectancias en la comparativa y la posibilidad de utilizar múltiples targets en vez de uno solo fijo.

Se han registrados altos valores de $\mathrm{R}^{2}$ para todas las bandas, a excepción de la banda 8 NIR de S2, la cual ha presentado ligeramente valores inferiores. La validación de la metodología de SNOs con otros métodos, han confirmado la utilización de esta técnica para la armonización e interoperabilidad de los datos de diferentes sensores de satélites.

El aumento de la incertidumbre en la comparativa entre bandas de la misma región espectral, pero con grandes diferencias en sus RSRs, hace necesario continuar investigando en este comportamiento. También sería interesante implementar una serie de adaptaciones en la metodología para la definición de cruce de trayectorias terrestres de los satélites. Para el caso de L8 y S2 debido a que sus planos orbitales corren casi paralelos durante largas distancias, el punto de intersección SNO podría 
redefinirse como un segmento, permitiendo conjuntos de datos de SNOs más grandes. La adaptación del punto central de la SNOs, junto con la modificación de los umbrales de nubosidad y la posible automatización de los outliers son puntos de mejora y adaptabilidad de la metodología a las características que posean los sensores a comparar.

Esta metodología global de SNOs presentada, debido a su agilidad en la implementación y operatibilidad, podría aplicarse en la evaluación de múltiples sensores de satélites, adaptando la misma a las características de las misiones a comparar.

\subsection{Anexos: Productos S2 y L8 utilizados}

En la Tabla 11 se muestran las características principales de los productos de S2 y L8 utilizados para el cálculo de las SNOs, con el umbral de 30 minutos de intervalo entre adquisiciones y el filtro de nubosidad del $5 \%$.

Tabla 11. Data set de SNOs con el umbral de 30 minutos de intervalo entre adquisiciones y el filtro de nubosidad del $5 \%$ aplicado.

\begin{tabular}{|c|c|c|c|}
\hline $\begin{array}{l}\text { Fecha de } \\
\text { Adquisición }\end{array}$ & Identificador del Producto S2 & Identificador del Producto L8 & $\begin{array}{c}\text { SNO } \\
\text { Intersección } \\
\text { Lon, Lat }\left({ }^{\circ}\right)\end{array}$ \\
\hline 2015-08-12 & S2A_MSIL1C_20150812T104026_N0204_R008_T31TEJ_20150812T104021 & LC08_L1TP_197030_20150812_20170406_01_T1 & $\begin{array}{l}3.4687 \\
43.5385\end{array}$ \\
\hline 2015-09-04 & S2A_MSIL1C_20150904T072816_N0204_R049_T39UXP_20150904T073107 & LC08_L1TP_166027_20150904_20170404_01_T1 & $\begin{array}{l}52.9557 \\
47.8579\end{array}$ \\
\hline 2015-11-15 & S2A_MSIL1C_20151115T163532_N0204_R083_T16TGQ_20151115T163534 & LC08_L1TP_021029_20151115_20170225_01_T1 & $\begin{array}{c}-84.1691 \\
44.8334\end{array}$ \\
\hline 2015-12-04 & S2A_MSIL1C_20151204T170702_N0204_R069_T14RQU_20151204T171455 & LC08_L1TP_026039_20151204_20170224_01_T1 & $\begin{array}{c}-96.4111 \\
29.8870\end{array}$ \\
\hline 2016-01-23 & S2A_MSIL1C_20160123T052112_N0201_R062_T43QGC_20160123T052434 & LC08_L1TP_145046_20160123_20170405_01_T1 & $\begin{array}{l}77.3742 \\
20.3053\end{array}$ \\
\hline 2016-02-07 & S2A_MSIL1C_20160207T112012_NO201_R137_T29RMJ_20160207T112209 & LC08_L1TP_202042_20160207_20170330_01_T1 & $\begin{array}{l}-9.4531 \\
25.4921\end{array}$ \\
\hline 2016-03-28 & S2A_MSIL1C_20160328T060612_NO201_R134_T47XNE_20160328T060634 & LC08_L1TP_152006_20160328_20170327_01_T1 & $\begin{array}{c}102.5021 \\
75.9571\end{array}$ \\
\hline 2016-04-23 & S2A_MSIL1C_20160423T163322_N0201_R083_T16TFN_20160423T163610 & LC08_L1TP_021030_20160423_20170223_01_T1 & $\begin{array}{c}-84.9030 \\
42.8084\end{array}$ \\
\hline 2016-05-05 & S2A_MSIL1C_20160505T004712_NO202_R102_T54HTK_20160505T004953 & LC08_L1TP_098082_20160505_20170325_01_T1 & $\begin{array}{c}138.1499 \\
-31.9755\end{array}$ \\
\hline 2016-05-16 & S2A_MSIL1C_20160516T145922_N0202_R125_T22WFU_20160516T150205 & LC08_L1TP_006013_20160516_20170324_01_T1 & $\begin{array}{c}-48.4028 \\
66.4414\end{array}$ \\
\hline 2016-06-08 & S2A_MSIL1C_20160608T101032_N0202_R022_T33UWV_20160608T101220 & LC08_L1TP_192023_20160608_20170324_01_T1 & $\begin{array}{l}15.4202 \\
53.8655\end{array}$ \\
\hline 2016-06-23 & S2A_MSIL1C_20160623T142012_N0204_R096_T26XMG_20160623T142007 & LC08_L1TP_233008_20160623_20170323_01_T1 & $\begin{array}{c}-28.0386 \\
73.2500\end{array}$ \\
\hline 2016-06-27 & S2A_MSIL1C_20160627T085602_N0204_R007_T33MWM_20160627T091503 & LC08_L1TP_181066_20160627_20170323_01_T1 & $\begin{array}{c}15.5755 \\
-8.1661\end{array}$ \\
\hline 2016-08-04 & S2A_MSIL1C_20160804T145922_N0204_R125_T22WFU_20160804T145917 & LC08_L1TP_006013_20160804_20170322_01_T1 & $\begin{array}{c}-48.1918 \\
66.6366\end{array}$ \\
\hline 2016-08-27 & S2A_MSIL1C_20160827T101022_N0204_R022_T33UWV_20160827T101025 & LC08_L1TP_192023_20160827_20170321_01_T1 & $\begin{array}{l}15.2991 \\
53.6280\end{array}$ \\
\hline 2016-09-04 & S2A_MSIL1C_20160904T024542_N0204_R132_T53WNR_20160904T024545 & LC08_L1TP_120012_20160904_20170321_01_T1 & $\begin{array}{c}137.3442 \\
68.1469\end{array}$ \\
\hline 2016-10-12 & S2A_MSIL1C_20161012T004702_NO204_R102_T54JUR_20161012T004954 & LC08_L1TP_098079_20161012_20170319_01_T1 & $\begin{array}{c}139.5657 \\
-26.7139\end{array}$ \\
\hline 2016-11-15 & S2A_MSIL1C_20161115T083222_N0204_R021_T35PNP_20161115T084140 & LC08_L1TP_176051_20161115_20170318_01_T1 & $\begin{array}{l}27.7421 \\
12.6077\end{array}$ \\
\hline 2016-12-08 & S2A_MSIL1C_20161208T052212_N0204_R062_T430GC_20161208T052504 & LC08_L1TP_145046_20161208_20170317_01_T1 & $\begin{array}{l}77.2887 \\
19.9503\end{array}$ \\
\hline 2016-12-08 & S2A_MSIL1C_20161208T070252_N0204_R063_T41UNB_20161208T070254 & LC08_L1TP_161021_20161208_20170317_01_T1 & $\begin{array}{l}63.9491 \\
55.1862\end{array}$ \\
\hline 2016-12-19 & S2A_MSIL1C_20161219T163702_N0204_R083_T16TGQ_20161219T163834 & LC08_L1TP_021029_20161219_20170218_01_T1 & $\begin{array}{c}-84.3506 \\
44.3429\end{array}$ \\
\hline 2017-01-07 & S2A_MSIL1C_20170107T170701_N0204_R069_T14RQT_20170107T170831 & LC08_L1TP_026040_20170107_20170218_01_T1 & $\begin{array}{c}-96.6369 \\
29.0480\end{array}$ \\
\hline 2017-02-07 & S2A_MSIL1C_20170207T063021_N0204_R077_T43VEJ_20170207T063023 & LC08_L1TP_156017_20170207_20170216_01_T1 & $\begin{array}{l}75.5459 \\
61.5073\end{array}$ \\
\hline 2017-02-11 & S2A_MSIL1C_20170211T024831_N0204_R132_T53WNQ_20170211T024828 & LC08_L1TP_120013_20170211_20170217_01_T1 & $\begin{array}{c}\text { 136.3952, } \\
67.3552\end{array}$ \\
\hline
\end{tabular}




\begin{tabular}{|c|c|c|c|}
\hline 2017-03-13 & S2A_MSIL1C_20170313T110831_N0204_R137_T29RMJ_20170313T111212 & LC08_L1TP_202042_20170313_20170328_01_T1 & $\begin{array}{l}-9.4439 \\
25.5280\end{array}$ \\
\hline 2017-03-28 & S2A_MSIL1C_20170328T170301_N0204_R069_T14RQT_20170328T170619 & LC08_L1TP_026040_20170328_20170414_01_T1 & $\begin{array}{c}-96.5164 \\
29.4971\end{array}$ \\
\hline 2017-04-05 & S2A_MSIL1C_20170405T075611_N0204_R035_T37REQ_20170405T081035 & LC08_L1TP_171038_20170405_20170414_01_T1 & $\begin{array}{l}39.9037 \\
31.2414\end{array}$ \\
\hline 2017-04-24 & S2A_MSIL1C_20170424T082601_N0204_R021_T35PNP_20170424T083830 & LC08_L1TP_176051_20170424_20170502_01_T1 & $\begin{array}{l}27.6998, \\
12.4221\end{array}$ \\
\hline 2017-05-13 & S2A_MSIL1C_20170513T090021_N0205_R007_T33MWM_20170513T092026 & LC08_L1TP_181065_20170513_20170525_01_T1 & $\begin{array}{l}15.6498 \\
-7.8332 \\
\end{array}$ \\
\hline 2017-06-01 & S2A_MSIL1C_20170601T110651_N0205_R137_T29RMJ_20170601T111225 & LC08_L1TP_202042_20170601_20170615_01_T1 & $\begin{array}{l}-9.3525 \\
25.8857\end{array}$ \\
\hline 2017-06-09 & S2A_MSIL1C_20170609T004711_N0205_R102_T54JUQ_20170609T005308 & LC08_L1TP_098079_20170609_20170616_01_T1 & $\begin{array}{l}\text { 139.3032, } \\
-27.7203\end{array}$ \\
\hline 2017-06-24 & S2A_MSIL1C_20170624T075611_N0205_R035_T37SFS_20170624T075954 & LC08_L1TP_171037_20170624_20170701_01_T1 & $\begin{array}{l}40.3766, \\
32.9272\end{array}$ \\
\hline 2017-07-13 & S2A_MSIL1C_20170713T150911_N0205_R025_T25XEF_20170713T150911 & LC08_L1TP_007005_20170713_20170726_01_T1 & $\begin{array}{c}-31.3113 \\
76.5751 \\
\end{array}$ \\
\hline 2017-07-15 & S2B_MSIL1C_20170715T081609_N0205_R121_T38VNL_20170715T081603 & LC08_L1TP_174019_20170715_20170727_01_T1 & $\begin{array}{l}46.2853 \\
59.3029\end{array}$ \\
\hline 2017-07-28 & S2A_MSIL1C_20170728T155901_N0205_R097_T22XDG_20170728T160023 & LC08_L1TP_016008_20170728_20170810_01_T1 & $\begin{array}{c}-52.9801, \\
73.3561\end{array}$ \\
\hline 2017-08-01 & S2A_MSIL1C_20170801T140021_N0205_R010_T26WNE_20170801T140016 & LC08_L1TP_229009_20170801_20170811_01_T1 & $\begin{array}{c}-25.5732 \\
71.9617\end{array}$ \\
\hline 2017-08-14 & S2B_MSIL1C_20170814T183309_N0205_R127_T11SPV_20170814T183307 & LC08_L1TP_039035_20170814_20170825_01_T1 & $\begin{array}{c}-114.9258 \\
35.6075\end{array}$ \\
\hline 2017-08-20 & S2A_MSIL1C_20170820T160901_N0205_R140_T22XDH_20170820T160902 & LC08_L1TP_017007_20170820_20170826_01_T1 & $\begin{array}{c}-53.0172 \\
74.4073\end{array}$ \\
\hline 2017-08-20 & S2A_MSIL1C_20170820T110651_N0205_R137_T29RMJ_20170820T111220 & LC08_L1TP_202042_20170820_20170826_01_T1 & $\begin{array}{l}-9.2816 \\
26.1619 \\
\end{array}$ \\
\hline 2017-08-22 & S2B_MSIL1C_20170822T141949_N0205_R096_T27XVD_20170822T141947 & LC08_L1TP_232007_20170822_20170911_01_T1 & $\begin{array}{c}-22.9999 \\
75.2631\end{array}$ \\
\hline 2017-08-28 & S2A_MSIL1C_20170828T004711_NO205_R102_T54JUR_20170828T005307 & LC08_L1TP_098078_20170828_20170914_01_T1 & $\begin{array}{l}139.6069, \\
-26.5550\end{array}$ \\
\hline 2017-09-04 & S2A_MSIL1C_20170904T165851_N0205_R069_T14RQV_20170904T170402 & LC08_L1TP_026039_20170904_20180125_01_T1 & $\begin{array}{c}-96.1340 \\
30.9020\end{array}$ \\
\hline 2017-09-10 & S2B_MSIL1C_20170910T095019_N0205_R079_T32QNJ_20170910T100356 & LC08_L1TP_189045_20170910_20170927_01_T1 & $\begin{array}{l}9.5894 \\
21.0792 \\
\end{array}$ \\
\hline 2017-09-12 & S2A_MSIL1C_20170912T075611_N0205_R035_T37SFT_20170912T075950 & LC08_L1TP_171037_20170912_20170928_01_T1 & $\begin{array}{l}40.6307 \\
33.8132\end{array}$ \\
\hline 2017-09-20 & S2A_MSIL1C_20170920T021601_N0205_R003_T55WEU_20170920T021627 & LC08_L1TP_115010_20170920_20170930_01_T1 & $\begin{array}{c}148.6628 \\
70.8084\end{array}$ \\
\hline 2017-09-25 & S2B_MSIL1C_20170925T142029_N0205_R010_T2OJKN_20170925T142023 & LC08_L1TP_230081_20170925_20180528_01_T1 & $\begin{array}{l}-65.1780 \\
-29.8396\end{array}$ \\
\hline 2017-10-20 & S2A_MSIL1C_20171020T090021_NO205_R007_T33LVH_20171020T091816 & LC08_L1TP_181068_20171020_20171106_01_T1 & $\begin{array}{l}\text { 14.9176, } \\
-11.0946\end{array}$ \\
\hline 2017-10-22 & S2B_MSIL1C_20171022T071249_N0205_R106_T38NPK_20171022T072130 & LC08_L1TP_163057_20171022_20171107_01_T1 & $\begin{array}{c}45.9595 \\
4.0151 \\
\end{array}$ \\
\hline 2017-11-08 & S2A_MSIL1C_20171108T111251_N0206_R137_T29QMG_20171108T145151 & LC08_L1TP_202043_20171108_20171121_01_T1 & $\begin{array}{l}-9.8014 \\
24.1148\end{array}$ \\
\hline 2017-11-29 & S2B_MSIL1C_20171129T095339_N0206_R079_T32QNJ_20171129T115638 & LC08_L1TP_189046_20171129_20171207_01_T1 & $\begin{array}{l}9.5698 \\
20.9984\end{array}$ \\
\hline 2017-11-29 & S2B_MSIL1C_20171129T113419_N0206_R080_T30UVG_20171129T133534 & LC08_L1TP_205021_20171129_20171207_01_T1 & $\begin{array}{l}-3.7183 \\
55.7397\end{array}$ \\
\hline 2017-11-29 & S2B_MSIL1C_20171129T081249_N0206_R078_T34HFK_20171129T115343 & LC08_L1TP_173082_20171129_20171207_01_T1 & $\begin{array}{l}22.2712 \\
-32.2408 \\
\end{array}$ \\
\hline 2017-12-01 & S2A_MSIL1C_20171201T080301_NO206_R035_T37SFS_20171201T100357 & LC08_L1TP_171037_20171201_20171207_01_T1 & $\begin{array}{l}40.4777 \\
33.2813\end{array}$ \\
\hline 2018-01-27 & S2A_MSIL1C_20180127T111321_N0206_R137_T29RMK_20180127T162747 & LC08_L1TP_202042_20180127_20180207_01_T1 & $\begin{array}{l}-9.1689 \\
26.5989\end{array}$ \\
\hline 2018-01-29 & S2B_MSIL1C_20180129T092229_NO206_R093_T34SEH_20180129T112249 & LC08_L1TP_184034_20180129_20180207_01_T1 & $\begin{array}{l}21.7305, \\
37.9342\end{array}$ \\
\hline 2018-02-17 & S2B_MSIL1C_20180217T081009_N0206_R078_T34JFL_20180217T121107 & LC08_L1TP_173082_20180217_20180307_01_T1 & $\begin{array}{l}22.5676 \\
-31.1783\end{array}$ \\
\hline 2018-03-04 & S2B_MSIL1C_20180304T142029_N0206_R010_T20JLR_20180304T191354 & LC08_L1TP_230079_20180304_20180319_01_T1 & $\begin{array}{l}-64.3401 \\
-26.6743\end{array}$ \\
\hline 2018-03-10 & S2A_MSIL1C_20180310T082751_NO206_R021_T35PPS_20180310T122012 & LC08_L1TP_176050_20180310_20180320_01_T1 & $\begin{array}{l}28.2813 \\
14.9583\end{array}$ \\
\hline 2018-03-12 & S2B_MSIL1C_20180312T063639_N0206_R120_T40RGR_20180312T102023 & LC08_L1TP_158041_20180312_20180320_01_T1 & $\begin{array}{l}59.1391 \\
27.8870\end{array}$ \\
\hline 2018-03-31 & S2B_MSIL1C_20180331T070619_N0206_R106_T38NPP_20180331T100829 & LC08_L1TP_163055_20180331_20180405_01_T1 & $\begin{array}{c}\text { 46.7244, } \\
7.4643\end{array}$ \\
\hline 2018-04-02 & S2A_MSIL1C_20180402T051651_N0206_R062_T43QGD_20180402T090406 & LC08_L1TP_145046_20180402_20180416_01_T1 & $\begin{array}{l}77.5245 \\
20.9270 \\
\end{array}$ \\
\hline 2018-04-11 & S2B_MSIL1C_20180411T181919_NO206_R127_T11SPT_20180411T220513 & LC08_L1TP_039036_20180411_20180417_01_T1 & $\begin{array}{c}-115.3518 \\
34.1632\end{array}$ \\
\hline 2018-04-17 & S2A_MSIL1C_20180417T110651_N0206_R137_T29RMH_20180417T164957 & LC08_L1TP_202043_20180417_20180501_01_T1 & $\begin{array}{l}-9.5650 \\
25.0522\end{array}$ \\
\hline 2018-04-25 & S2A_MSIL1C_20180425T004711_NO206_R102_T54JUT_20180425T021141 & LC08_L1TP_098077_20180425_20180502_01_T1 & $\begin{array}{c}140.0578 \\
-24.7916\end{array}$ \\
\hline 2018-05-08 & S2B_MSIL1C_20180508T080609_N0206_R078_T34HFK_20180508T133204 & LC08_L1TP_173082_20180508_20180517_01_T1 & $\begin{array}{l}22.2174 \\
-32.4317\end{array}$ \\
\hline 2018-05-10 & S2A_MSIL1C_20180510T094031_N0206_R036_T35VME_20180510T114819 & LC08_L1TP_187019_20180510_20180517_01_T1 & $\begin{array}{l}25.3924 \\
58.1392\end{array}$ \\
\hline
\end{tabular}




\begin{tabular}{|c|c|c|c|}
\hline 2018-05-10 & S2A_MSIL1C_20180510T043701_N0206_R033_T50XNG_20180510T074003 & LC08_L1TP_139008_20180510_20180517_01_T1 & $\begin{array}{c}117.6388 \\
73.0903\end{array}$ \\
\hline 2018-05-12 & S2B_MSIL1C_20180512T074729_N0206_R135_T4OVDP_20180512T113937 & LC08_L1TP_169017_20180512_20180517_01_T1 & $\begin{array}{l}55.7179 \\
61.9131\end{array}$ \\
\hline 2018-05-16 & S2B_MSIL1C_20180516T022549_NO206_R046_T52UFE_20180516T040424 & LC08_L1TP_117022_20180516_20180604_01_T1 & $\begin{array}{c}131.2951 \\
54.0036\end{array}$ \\
\hline 2018-05-16 & S2B_MSIL1C_20180516T040539_N0206_R047_T50WMV_20180516T070218 & LC08_L1TP_133013_20180516_20180604_01_T1 & $\begin{array}{c}116.1178 \\
67.2360\end{array}$ \\
\hline 2018-05-23 & S2B_MSIL1C_20180523T142039_N0206_R010_T20JLP_20180523T192205 & LC08_L1TP_230080_20180523_20180605_01_T1 & $\begin{array}{l}-64.8003 \\
-28.4302\end{array}$ \\
\hline 2018-05-23 & S2B_MSIL1C_20180523T204019_N0206_R014_T10XDG_20180524T001026 & LC08_L1TP_061008_20180523_20180605_01_T1 & $\begin{array}{c}-123.9418 \\
73.1388\end{array}$ \\
\hline 2018-06-04 & S2B_MSIL1C_20180604T043659_N0206_R033_T48WXU_20180604T081821 & LC08_L1TP_138013_20180604_20180615_01_T1 & $\begin{array}{c}107.7603 \\
66.5143\end{array}$ \\
\hline 2018-06-06 & S2A_MSIL1C_20180606T024651_N0206_R132_T53WPS_20180606T040212 & LC08_L1TP_120011_20180606_20180615_01_T1 & $\begin{array}{c}138.5057 \\
69.0425\end{array}$ \\
\hline 2018-06-07 & S2B_MSIL1C_20180607T180919_N0206_R084_T17XMD_20180607T213729 & LC08_L1TP_038006_20180607_20180615_01_T1 & $\begin{array}{c}-80.8364 \\
75.2836\end{array}$ \\
\hline 2018-06-11 & S2B_MSIL1C_20180611T174909_NO206_R141_T13UFR_20180611T213053 & LC08_L1TP_034025_20180611_20180615_01_T1 & $\begin{array}{c}-102.1482 \\
50.1601\end{array}$ \\
\hline 2018-06-13 & S2A_MSIL1C_20180613T155901_N0206_R097_T19VCC_20180613T194300 & LC08_L1TP_016021_20180613_20180703_01_T1 & $\begin{array}{c}-71.3207 \\
56.3924\end{array}$ \\
\hline 2018-06-23 & S2B_MSIL1C_20180623T020449_N0206_R017_T51KTT_20180623T033510 & LC08_L1TP_111074_20180623_20180703_01_T1 & $\begin{array}{c}120.9584 \\
-20.5823\end{array}$ \\
\hline 2018-06-30 & S2B_MSIL1C_20180630T181919_N0206_R127_T11SQA_20180630T232219 & LC08_L1TP_039035_20180630_20180716_01_T1 & $\begin{array}{c}-114.5805 \\
36.7495\end{array}$ \\
\hline 2018-07-02 & S2A_MSIL1C_20180702T150721_N0206_R082_T19MBT_20180702T195445 & LC08_L1TP_005062_20180702_20180716_01_T1 & $\begin{array}{c}-71.3037 \\
-2.6055\end{array}$ \\
\hline 2018-07-02 & S2A_MSIL1C_20180702T162901_N0206_R083_T16TGS_20180702T214026 & LC08_L1TP_021028_20180702_20180716_01_T1 & $\begin{array}{c}-83.6504 \\
46.1967\end{array}$ \\
\hline 2018-07-10 & S2A_MSIL1C_20180710T072621_NO206_R049_T39UXP_20180710T085441 & LC08_L1TP_166027_20180710_20180717_01_T1 & $\begin{array}{l}53.0450 \\
48.0755\end{array}$ \\
\hline 2018-07-12 & S2B_MSIL1C_20180712T053639_N0206_R005_T44UPF_20180712T092034 & LC08_L1TP_148022_20180712_20180717_01_T1 & $\begin{array}{l}83.8405 \\
54.7237\end{array}$ \\
\hline 2018-07-14 & S2A_MSIL1C_20180714T004711_N0206_R102_T54JUR_20180714T021605 & LC08_L1TP_098078_20180714_20180730_01_T1 & $\begin{array}{c}139.6443 \\
-26.4100\end{array}$ \\
\hline 2018-07-27 & S2B_MSIL1C_20180727T095029_N0206_R079_T32QNK_20180727T135801 & LC08_L1TP_189045_20180727_20180731_01_T1 & $\begin{array}{l}9.8901 \\
22.3097\end{array}$ \\
\hline 2018-07-29 & S2A_MSIL1C_20180729T075611_N0206_R035_T37SFU_20180729T092130 & LC08_L1TP_171036_20180729_20180813_01_T1 & $\begin{array}{l}40.9243 \\
34.8195\end{array}$ \\
\hline 2018-07-29 & S2A_MSIL1C_20180729T094031_N0206_R036_T35VNJ_20180729T101505 & LC08_L1TP_187017_20180729_20180813_01_T1 & $\begin{array}{l}27.7539 \\
61.5600\end{array}$ \\
\hline 2018-07-31 & S2B_MSIL1C_20180731T060629_N0206_R134_T42TYQ_20180731T084741 & LC08_L1TP_153029_20180731_20180814_01_T1 & $\begin{array}{l}71.8012 \\
44.5272\end{array}$ \\
\hline 2018-08-11 & S2B_MSIL1C_20180811T142029_NO206_R010_T20JKN_20180811T194747 & LC08_L1TP_230081_20180811_20180815_01_T1 & $\begin{array}{l}-65.0898 \\
-29.5130\end{array}$ \\
\hline 2018-08-19 & S2B_MSIL1C_20180819T063619_NO206_R120_T40RGS_20180819T093637 & LC08_L1TP_158040_20180819_20180829_01_T1 & $\begin{array}{l}\text { 59.2724, } \\
28.3919\end{array}$ \\
\hline 2018-08-21 & S2A_MSIL1C_20180821T044701_N0206_R076_T45TYE_20180821T075342 & LC08_L1TP_140033_20180821_20180829_01_T1 & $\begin{array}{l}90.2648, \\
39.6924\end{array}$ \\
\hline 2018-09-07 & S2B_MSIL1C_20180907T070609_NO206_R106_T38NPP_20180907T110607 & LC08_L1TP_163055_20180907_20180912_01_T1 & $\begin{array}{c}46.8043 \\
7.8230\end{array}$ \\
\hline 2018-09-11 & S2B_MSIL1C_20180911T020439_N0206_R017_T51KUU_20180911T052025 & LC08_L1TP_111074_20180911_20180927_01_T1 & $\begin{array}{c}121.1695 \\
-19.7059\end{array}$ \\
\hline 2018-09-11 & S2B_MSIL1C_20180911T032529_N0206_R018_T49SCU_20180911T070657 & LC08_L1TP_127036_20180911_20180927_01_T1 & $\begin{array}{c}108.9604 \\
35.0366\end{array}$ \\
\hline 2018-09-13 & S2A_MSIL1C_20180913T031541_N0206_R118_T51WXN_20180913T051046 & LC08_L1TP_125015_20180913_20180927_01_T1 & $\begin{array}{c}126.3121 \\
64.9526\end{array}$ \\
\hline 2018-09-18 & S2B_MSIL1C_20180918T182009_NO206_R127_T11SQB_20180918T221717 & LC08_L1TP_039034_20180918_20180928_01_T1 & $\begin{array}{c}-114.4716 \\
37.1044\end{array}$ \\
\hline 2018-09-20 & S2A_MSIL1C_20180920T150721_N0206_R082_T19MBU_20180920T184627 & LC08_L1TP_005061_20180920_20180928_01_T1 & $\begin{array}{c}-71.0166 \\
-1.3029\end{array}$ \\
\hline 2018-09-24 & S2A_MSIL1C_20180924T110801_N0206_R137_T29RNL_20180924T152333 & LC08_L1TP_202041_20180924_20180929_01_T1 & $\begin{array}{l}-8.9195 \\
27.5574\end{array}$ \\
\hline 2018-09-26 & S2B_MSIL1C_20180926T073639_N0206_R092_T36LXK_20180926T113524 & LC08_L1TP_168070_20180926_20181009_01_T1 & $\begin{array}{l}34.2997 \\
-14.4166\end{array}$ \\
\hline 2018-09-28 & S2A_MSIL1C_20180928T072651_N0206_R049_T39TXN_20180928T141734 & LC08_L1TP_166027_20180928_20181009_01_T1 & $\begin{array}{l}52.7750 \\
47.4132\end{array}$ \\
\hline 2018-09-30 & S2B_MSIL1C_20180930T053639_NO206_R005_T44UPE_20180930T092159 & LC08_L1TP_148022_20180930_20181010_01_T1 & $\begin{array}{l}83.4770 \\
54.0308\end{array}$ \\
\hline 2018-10-02 & S2A_MSIL1C_20181002T004701_N0206_R102_T54JUR_20181002T022020 & LC08_L1TP_098078_20181002_20181010_01_T1 & $\begin{array}{c}139.6300 \\
-26.4656\end{array}$ \\
\hline 2018-10-09 & S2A_MSIL1C_20181009T184251_N0206_R070_T12VWM_20181009T222044 & LC08_L1TP_042018_20181009_20181029_01_T1 & $\begin{array}{c}-109.5548 \\
59.6649\end{array}$ \\
\hline 2018-10-11 & S2B_MSIL1C_20181011T135109_N0206_R024_T22LBP_20181011T172553 & LC08_L1TP_225067_20181011_20181030_01_T1 & $\begin{array}{l}-52.9522 \\
-10.6420\end{array}$ \\
\hline 2018-10-15 & S2B_MSIL1C_20181015T113319_N0206_R080_T30UVG_20181015T133405 & LC08_L1TP_205021_20181015_20181030_01_T1 & $\begin{array}{l}-3.7972 \\
55.5972\end{array}$ \\
\hline 2018-11-05 & S2A_MSIL1C_20181105T083121_N0206_R021_T35PNP_20181105T100715 & LC08_L1TP_176052_20181105_20181115_01_T1 & $\begin{array}{l}27.5869 \\
11.9251\end{array}$ \\
\hline 2018-11-07 & S2B_MSIL1C_20181107T064049_N0207_R120_T40RFP_20181107T103500 & LC08_L1TP_158042_20181107_20181116_01_T1 & $\begin{array}{l}58.6302 \\
25.9275\end{array}$ \\
\hline 2018-11-09 & S2A_MSIL1C_20181109T063051_N0207_R077_T43VEJ_20181109T083632 & LC08_L1TP_156017_20181109_20181116_01_T1 & $\begin{array}{l}75.9871 \\
62.0783\end{array}$ \\
\hline
\end{tabular}




\begin{tabular}{|c|c|c|c|}
\hline 2018-11-11 & S2B_MSIL1C_20181111T025939_N0207_R032_T50TQS_20181111T055543 & LC08_L1TP_122028_20181111_20181127_01_T1 & $\begin{array}{c}120.4116 \\
46.5705\end{array}$ \\
\hline 2018-11-28 & S2A_MSIL1C_20181128T052141_N0207_R062_T43QGD_20181128T090704 & LC08_L1TP_145046_20181128_20181211_01_T1 & $\begin{array}{l}77.4906 \\
20.7869\end{array}$ \\
\hline 2018-11-30 & S2B_MSIL1C_20181130T020439_N0207_R017_T51KTT_20181130T060546 & LC08_L1TP_111074_20181130_20181211_01_T1 & $\begin{array}{c}120.9916 \\
-20.4450\end{array}$ \\
\hline 2018-12-28 & S2A_MSIL1C_20181228T170711_N0207_R069_T14RQV_20181228T202923 & LC08_L1TP_026039_20181228_20190129_01_T1 & $\begin{array}{c}-96.0964 \\
31.0386\end{array}$ \\
\hline 2019-01-03 & S2B_MSIL1C_20190103T095409_N0207_R079_T32QNJ_20190103T115034 & LC08_L1TP_189045_20190103_20190130_01_T1 & $\begin{array}{l}9.6094 \\
21.1616\end{array}$ \\
\hline 2019-01-03 & S2B_MSIL1C_20190103T081329_N0207_R078_T34HFK_20190103T102420 & LC08_L1TP_173082_20190103_20190130_01_T1 & $\begin{array}{l}22.2585 \\
-32.2861\end{array}$ \\
\hline 2019-01-24 & S2A_MSIL1C_20190124T083231_N0207_R021_T35PPT_20190124T095836 & LC08_L1TP_176049_20190124_20190205_01_T1 & $\begin{array}{l}28.4836 \\
15.8312\end{array}$ \\
\hline 2019-01-28 & S2A_MSIL1C_20190128T063121_N0207_R077_T43VEK_20190128T075200 & LC08_L1TP_156016_20190128_20190206_01_T1 & $\begin{array}{l}76.2697 \\
62.4334 \\
\end{array}$ \\
\hline 2019-02-14 & S2B_MSIL1C_20190214T071009_N0207_R106_T38PQQ_20190214T104949 & LC08_L1TP_163054_20190214_20190222_01_T1 & $\begin{array}{c}\text { 47.0368, } \\
8.8635\end{array}$ \\
\hline 2019-02-20 & S2A_MSIL1C_20190220T031751_N0207_R118_T51VXL_20190220T050828 & LC08_L1TP_125015_20190220_20190222_01_T1 & $\begin{array}{c}125.3264 \\
63.8829\end{array}$ \\
\hline 2019-03-03 & S2A_MSIL1C_20190303T110951_N0207_R137_T29RML_20190303T132419 & LC08_L1TP_202041_20190303_20190309_01_T1 & $\begin{array}{l}-9.0120 \\
27.2033\end{array}$ \\
\hline 2019-03-11 & S2A_MSIL1C_20190311T004701_N0207_R102_T54KVV_20190311T022013 & LC08_L1TP_098076_20190311_20190325_01_T1 & $\begin{array}{c}140.4117 \\
-23.3815\end{array}$ \\
\hline 2019-04-08 & S2B_MSIL1C_20190408T142039_N0207_R010_T20JLP_20190408T174012 & LC08_L1TP_230080_20190408_20190422_01_T1 & $\begin{array}{l}-64.7462 \\
-28.2261\end{array}$ \\
\hline 2019-05-05 & S2B_MSIL1C_20190505T084609_N0207_R107_T36UWB_20190505T111007 & LC08_L1TP_179025_20190505_20190520_01_T1 & $\begin{array}{l}34.1044 \\
50.8476\end{array}$ \\
\hline 2019-05-07 & S2A_MSIL1C_20190507T051651_N0207_R062_T43QGD_20190507T085455 & LC08_L1TP_145045_20190507_20190521_01_T1 & $\begin{array}{l}77.6001 \\
21.2382\end{array}$ \\
\hline 2019-05-22 & S2A_MSIL1C_20190522T160911_N0207_R140_T22XDH_20190522T212646 & LC08_L1TP_017007_20190522_20190604_01_T1 & $\begin{array}{c}-52.6242 \\
74.5578\end{array}$ \\
\hline 2019-05-22 & S2A_MSIL1C_20190522T110621_N0207_R137_T29RMH_20190522T181102 & LC08_L1TP_202043_20190522_20190604_01_T1 & $\begin{array}{l}-9.5346 \\
25.1720\end{array}$ \\
\hline 2019-05-30 & S2A_MSIL1C_20190530T004711_NO207_R102_T54JUP_20190530T022148 & LC08_L1TP_098080_20190530_20190605_01_T1 & $\begin{array}{c}139.0652 \\
-28.6207\end{array}$ \\
\hline 2019-06-06 & S2A_MSIL1C_20190606T165901_N0207_R069_T14RQT_20190606T220932 & LC08_L1TP_026040_20190606_20190619_01_T1 & $\begin{array}{c}-96.5873, \\
29.2330\end{array}$ \\
\hline 2019-06-08 & S2B_MSIL1C_20190608T215539_N0207_R029_T06WVC_20190608T233549 & LC08_L1TP_072011_20190608_20190619_01_T1 & $\begin{array}{c}-147.4049 \\
69.8160\end{array}$ \\
\hline 2019-06-12 & S2B_MSIL1C_20190612T095039_N0207_R079_T32QMF_20190612T120554 & LC08_L1TP_189047_20190612_20190619_01_T1 & $\begin{array}{c}9.0059 \\
18.6496\end{array}$ \\
\hline 2019-06-14 & S2A_MSIL1C_20190614T075611_N0207_R035_T37SER_20190614T092644 & LC08_L1TP_171038_20190614_20190620_01_T1 & $\begin{array}{l}\text { 40.0994, } \\
31.9447\end{array}$ \\
\hline 2019-06-22 & S2A_MSIL1C_20190622T053651_N0207_R005_T48XVG_20190622T073519 & LC08_L1TP_147008_20190622_20190704_01_T1 & $\begin{array}{c}103.8639 \\
73.6931\end{array}$ \\
\hline 2019-06-25 & S2A_MSIL1C_20190625T141011_N0207_R053_T26XNG_20190625T142549 & LC08_L1TP_232008_20190625_20190705_01_T1 & $\begin{array}{c}-26.0279 \\
73.0120\end{array}$ \\
\hline 2019-06-27 & S2B_MSIL1C_20190627T142049_N0207_R010_T20JKL_20190627T173831 & LC08_L1TP_230081_20190627_20190705_01_T1 & $\begin{array}{l}-65.4539 \\
-30.8507\end{array}$ \\
\hline 2019-07-05 & S2B_MSIL1C_20190705T063639_N0207_R120_T40RFR_20190705T092912 & LC08_L1TP_158041_20190705_20190719_01_T1 & $\begin{array}{l}59.0271 \\
27.4600\end{array}$ \\
\hline 2019-07-07 & S2A_MSIL1C_20190707T044711_N0207_R076_T45SYD_20190707T074645 & LC08_L1TP_140033_20190707_20190719_01_T1 & $\begin{array}{l}90.0519 \\
39.0348\end{array}$ \\
\hline 2019-07-10 & S2A_MSIL1C_20190710T214541_N0208_R129_TO6WWB_20190710T232820 & LC08_L1TP_072011_20190710_20190719_01_T1 & $\begin{array}{c}-146.1640, \\
68.8994\end{array}$ \\
\hline 2019-07-18 & S2A_MSIL1C_20190718T155911_N0208_R097_T19VCC_20190718T194134 & LC08_L1TP_016021_20190718_20190731_01_T1 & $\begin{array}{c}-71.5169 \\
56.0478 \\
\end{array}$ \\
\hline 2019-07-20 & S2B_MSIL1C_20190720T054649_N0208_R048_T47XNA_20190720T092848 & LC08_L1TP_151008_20190720_20190731_01_T1 & $\begin{array}{l}99.4777 \\
72.8331\end{array}$ \\
\hline 2019-07-22 & S2A_MSIL1C_20190722T104031_N0208_R008_T31TEJ_20190722T110458 & LC08_L1TP_197030_20190722_20190801_01_T1 & $\begin{array}{l}3.4531 \\
43.4949\end{array}$ \\
\hline 2019-07-28 & S2B_MSIL1C_20190728T020459_N0208_R017_T51KUU_20190728T051808 & LC08_L1TP_111074_20190728_20190801_01_T1 & $\begin{array}{c}\text { 121.1611, } \\
-19.7412\end{array}$ \\
\hline 2019-07-30 & S2A_MSIL1C_20190730T063631_NO208_R120_T46XEK_20190730T075058 & LC08_L1TP_157006_20190730_20190801_01_T1 & $\begin{array}{l}94.6434 \\
75.8645\end{array}$ \\
\hline 2019-07-30 & S2A_MSIL1C_20190730T031541_N0208_R118_T51WXN_20190730T050828 & LC08_L1TP_125015_20190730_20190801_01_T1 & $\begin{array}{c}126.3051 \\
64.9453\end{array}$ \\
\hline 2019-07-30 & S2A_MSIL1C_20190730T063631_N0208_R120_T45XWB_20190730T075058 & LC08_L1TP_157008_20190730_20190801_01_T1 & $\begin{array}{l}88.5970 \\
73.6222\end{array}$ \\
\hline 2019-08-01 & S2B_MSIL1C_20190801T030549_N0208_R075_T54XWG_20190801T045652 & LC08_L1TP_123008_20190801_20190819_01_T1 & $\begin{array}{c}\text { 141.2919, } \\
73.5465\end{array}$ \\
\hline 2019-08-06 & S2A_MSIL1C_20190806T150721_N0208_R082_T19MBT_20190806T182907 & LC08_L1TP_005062_20190806_20190820_01_T1 & $\begin{array}{c}-71.1872 \\
-2.0768 \\
\end{array}$ \\
\hline 2019-08-10 & S2A_MSIL1C_20190810T160911_N0208_R140_T22XDH_20190810T193101 & LC08_L1TP_017007_20190810_20190820_01_T1 & $\begin{array}{c}-52.5442 \\
74.5882\end{array}$ \\
\hline 2019-08-12 & S2B_MSIL1C_20190812T092039_N0208_R093_T34SEH_20190812T113125 & LC08_L1TP_184033_20190812_20190820_01_T1 & $\begin{array}{l}21.9898 \\
38.7538\end{array}$ \\
\hline 2019-08-14 & S2A_MSIL1C_20190814T072621_N0208_R049_T39TXN_20190814T084311 & LC08_L1TP_166027_20190814_20190820_01_T1 & $\begin{array}{l}52.8605 \\
47.6248\end{array}$ \\
\hline 2019-08-18 & S2A_MSIL1C_20190818T004711_NO208_R102_T54JUS_20190818T021956 & LC08_L1TP_098078_20190818_20190902_01_T1 & $\begin{array}{c}\text { 139.7911, } \\
-25.8391\end{array}$ \\
\hline 2019-08-18 & S2A_MSIL1C_20190818T052651_N0208_R105_T47WNT_20190818T083140 & LC08_L1TP_146011_20190818_20190902_01_T1 & $\begin{array}{l}99.9548 \\
70.2496\end{array}$ \\
\hline
\end{tabular}




\begin{tabular}{|c|c|c|c|}
\hline 2019-08-31 & S2B_MSIL1C_20190831T095039_N0208_R079_T32QNJ_20190831T133329 & LC08_L1TP_189045_20190831_20190916_01_T1 & $\begin{array}{l}9.7013 \\
21.5386\end{array}$ \\
\hline 2019-09-02 & S2A_MSIL1C_20190902T075611_NO208_R035_T37SFT_20190902T100157 & LC08_L1TP_171036_20190902_20190916_01_T1 & $\begin{array}{l}40.7337 \\
34.1685\end{array}$ \\
\hline 2019-09-23 & S2B_MSIL1C_20190923T063629_N0208_R120_T40RFR_20190923T103632 & LC08_L1TP_158041_20190923_20190926_01_T1 & $\begin{array}{l}59.0156 \\
27.4162\end{array}$ \\
\hline 2019-09-27 & S2B_MSIL1C_20190927T043659_N0208_R033_T48WWT_20190927T072914 & LC08_L1TP_138014_20190927_20191017_01_T1 & $\begin{array}{c}106.7168 \\
65.5093\end{array}$ \\
\hline 2019-10-16 & S2B_MSIL1C_20191016T020019_N0208_R017_T51KTS_20191016T051826 & LC08_L1TP_111075_20191016_20191029_01_T1 & $\begin{array}{c}120.8903 \\
-20.8636\end{array}$ \\
\hline 2019-10-23 & S2B_MSIL1C_20191023T182419_N0208_R127_T11SPU_20191023T215755 & LC08_L1TP_039036_20191023_20191030_01_T1 & $\begin{array}{c}-115.1180 \\
34.9605\end{array}$ \\
\hline
\end{tabular}

\subsection{Referencias}

Anderson, N., Czapla-Myers, J., Leisso, N., Biggar, S., Burkhart, C., Kingston, R., \& Thome, K. (2013). Design and calibration of field deployable ground-viewing radiometers. Applied Optics, 52(2), 231-240.

Angal, A., Mishra, N., Xiong, X., \& Helder, D. (2014). Cross-calibration of Landsat 5 TM, and Landsat $8 \mathrm{OLI}$ with Aqua MODIS using PICS. In Earth Observing Systems XIX (Vol. 9218, p. 92180K). International Society for Optics and Photonics.

Barsi, J. A., Alhammoud, B., Czapla-Myers, J., Gascon, F., Haque, M. O., Kaewmanee, M., ... \& Markham, B. L. (2018). Sentinel-2A MSI and Landsat-8 OLI radiometric cross comparison over desert sites. European Journal of Remote Sensing, 51(1), 822837.

Bouvet, M. (2014). Radiometric comparison of multispectral imagers over a pseudoinvariant calibration site using a reference radiometric model. Remote sensing of environment, 140, 141-154.

Buchhorn, M., Smets, B., Bertels, L., Lesiv, M., Tsendbazar, N. E., Herold, M., \& Fritz, S. (2019). Copernicus Global Land Service: Land Cover 100m: Epoch 2015: Globe. Version V2. 0.2.

Cao, C., \& Heidinger, A. K. (2002). Intercomparison of the longwave infrared channels of MODIS and AVHRR/NOAA-16 using simultaneous nadir observations at orbit intersections. In Earth Observing Systems VII (Vol. 4814, pp. 306-316). International Society for Optics and Photonics.

Cao, C., Weinreb, M., \& Xu, H. (2004). Predicting simultaneous nadir overpasses among polar-orbiting meteorological satellites for the intersatellite calibration of radiometers. Journal of Atmospheric and Oceanic Technology, 21(4), 537-542.

Cao, C., Xu, H., Sullivan, J., McMillin, L., Ciren, P., \& Hou, Y. T. (2005). Intersatellite radiance biases for the High-Resolution Infrared Radiation Sounders (HIRS) on board NOAA-15,-16, and-17 from simultaneous nadir observations. Journal of Atmospheric and Oceanic Technology, 22(4), 381-395. 
Chander, G., Haque, M. O., Sampath, A., Brunn, A., Trosset, G., Hoffmann, D., ... \& Anderson, C. (2013). Radiometric and geometric assessment of data from the RapidEye constellation of satellites. International Journal of Remote Sensing, 34(16), 5905-5925.

Chander, G., Hewison, T. J., Fox, N., Wu, X., Xiong, X., \& Blackwell, W. J. (2013). Overview of intercalibration of satellite instruments. IEEE Transactions on Geoscience and Remote Sensing, 51(3), 1056-1080.

Chander, G., Mishra, N., Helder, D. L., Aaron, D. B., Angal, A., Choi, T., ... \& Doelling, D. R. (2012). Applications of spectral band adjustment factors (SBAF) for crosscalibration. IEEE Transactions on Geoscience and Remote Sensing, 51(3), 12671281.

Chander, G., Xiong, X. J., Choi, T. J., \& Angal, A. (2010). Monitoring on-orbit calibration stability of the Terra MODIS and Landsat 7 ETM+ sensors using pseudo-invariant test sites. Remote Sensing of Environment, 114(4), 925-939.

Claverie, M., Ju, J., Masek, J. G., Dungan, J. L., Vermote, E. F., Roger, J. C., ... \& Justice, C. (2018). The Harmonized Landsat and Sentinel-2 surface reflectance data set. Remote sensing of environment, 219, 145-161.

De Vries, C., Danaher, T., Denham, R., Scarth, P., \& Phinn, S. (2007). An operational radiometric calibration procedure for the Landsat sensors based on pseudoinvariant target sites. Remote Sensing of Environment, 107(3), 414-429.

ESA, $\quad$ Srd Sentinel-2 Validation Team Meeting. https://az659834.vo.msecnd.net/eventsairwesteuprod/production-nikalpublic/683987d4267640cba49b0a3e14b89a4e (Accecido el 22 de Junio de 2020d).

ESA, Sentinel 2 - Orbit. https://sentinel.esa.int/web/sentinel/missions/sentinel2/satellite-description/orbit (Accecido el 14 de Junio de 2020a).

ESA, Sentinel 2 Products Specification Document. https://sentinel.esa.int/documents/247904/349490/S2 MSI Product Specifica tion.pdf (Accecido el 18 de Junio de 2020b).

ESA, Sentinel 2. https://sentinel.esa.int/web/sentinel/missions/sentinel-2 (Accecido el 21 de Mayo de 2020).

ESA, Sentinel-2 L1C Data Quality Report. https://sentinel.esa.int/documents/247904/685211/Sentinel-

2 L1C Data Quality Report (Accecido el 22 de Junio de 2020c). 
Gascon, F., Bouzinac, C., Thépaut, O., Jung, M., Francesconi, B., Louis, J., ... \& Languille, F. (2017). Copernicus Sentinel-2A calibration and products validation status. Remote Sensing, 9(6), 584.

Gu, X., GUYOT, M., \& Verbrugghe, M. (1990). Analyse de la variabilité spatiale d'un sitetest. Exemple de la Crau (France). Photo interprétation (Paris), 29(1), 39-52

Hasan, M. N., Shrestha, M., Leigh, L., \& Helder, D. (2019). Evaluation of an Extended PICS (EPICS) for Calibration and Stability Monitoring of Optical Satellite Sensors. Remote Sensing, 11(15), 1755.

Heidinger, A. K., Cao, C., \& Sullivan, J. T. (2002). Using Moderate Resolution Imaging Spectrometer (MODIS) to calibrate advanced very high resolution radiometer reflectance channels. Journal of Geophysical Research: Atmospheres, 107(D23), AAC-11.

Helder, D. L., Basnet, B., \& Morstad, D. L. (2010). Optimized identification of worldwide radiometric pseudo-invariant calibration sites. Canadian Journal of Remote Sensing, 36(5), 527-539.

Helder, D. L., Karki, S., Bhatt, R., Micijevic, E., Aaron, D., \& Jasinski, B. (2011). Radiometric calibration of the Landsat MSS sensor series. IEEE Transactions on Geoscience and Remote Sensing, 50(6), 2380-2399.

Helder, D., Markham, B., Morfitt, R., Storey, J., Barsi, J., Gascon, F., ... \& Lewis, A. (2018). Observations and Recommendations for the Calibration of Landsat $8 \mathrm{OLI}$ and Sentinel 2 MSI for improved data interoperability. Remote Sensing, 10(9), 1340.

Helder, D., Thome, K. J., Mishra, N., Chander, G., Xiong, X., Angal, A., \& Choi, T. (2013). Absolute radiometric calibration of Landsat using a pseudo invariant calibration site. IEEE Transactions on Geoscience and Remote Sensing, 51(3), 1360-1369.

Irons, J. R., Dwyer, J. L., \& Barsi, J. A. (2012). The next Landsat satellite: The Landsat data continuity mission. Remote Sensing of Environment, 122, 11-21.

Kneubühler, M., Schaepman, M. E., Thome, K., \& Danesy, D. (2006). Long-term vicarious calibration efforts of MERIS at railroad valley playa (NV)-An update. ESA-SP, (615), online.

Lacherade, S., Fougnie, B., Henry, P., \& Gamet, P. (2013). Cross calibration over desert sites: Description, methodology, and operational implementation. IEEE Transactions on Geoscience and Remote Sensing, 51(3), 1098-1113.

Li, J., \& Roy, D. P. (2017). A global analysis of Sentinel-2A, Sentinel-2B and Landsat-8 data revisit intervals and implications for terrestrial monitoring. Remote Sensing, 9(9), 902. 
Liang, J. I., Piper, J., \& Tang, J. Y. (1989). Erosion and dilation of binary images by arbitrary structuring elements using interval coding. Pattern Recognition Letters, 9(3), 201-209.

Lillesand, T., Kiefer, R. W., \& Chipman, J. (2015). Remote sensing and image interpretation. John Wiley \& Sons.

Mishra, N., Haque, M. O., Leigh, L., Aaron, D., Helder, D., \& Markham, B. (2014). Radiometric cross calibration of Landsat 8 operational land imager (OLI) and Landsat 7 enhanced thematic mapper plus (ETM+). Remote sensing, 6(12), 12619-12638.

Mishra, N., Helder, D., Angal, A., Choi, J., \& Xiong, X. (2014). Absolute calibration of optical satellite sensors using Libya 4 pseudo invariant calibration site. Remote sensing, 6(2), 1327-1346.

NASA, Landsat 8. https://satellitesafety.gsfc.nasa.gov/landsat8.html (Accecido el 14 de Junio de 2020b)

NASA, Satellite Safety - Maneuver Types. https://satellitesafety.gsfc.nasa.gov/maneuvers.html (Accecido el 14 de Junio de 2020a)

Neigh, C. S., McCorkel, J., \& Middleton, E. M. (2015). Quantifying Libya-4 surface reflectance heterogeneity with WorldView-1, 2 and EO-1 Hyperion. IEEE Geoscience and Remote Sensing Letters, 12(11), 2277-2281.

NOAA, (2018). Advisory Committee on Commercial Remote Sensing (ACCRES). https://www.nesdis.noaa.gov/CRSRA/pdf/AACRES meeting 2018 Euroconsult. pdf (Accedido el 24 de Mayo de 2020).

Öhrwall, A. B., \& Isaksson, O. (2018). Product development challenges for space subsystem manufacturers. In DS 92: Proceedings of the DESIGN 2018 15th International Design Conference (pp. 1937-1944).

Piper, J. (1985). Efficient implementation of skeletonisation using interval coding. Pattern Recognition Letters, 3(6), 389-397.

Ruf, C. S., Chew, C., Lang, T., Morris, M. G., Nave, K., Ridley, A., \& Balasubramaniam, R. (2018). A new paradigm in earth environmental monitoring with the CYGNSS small satellite constellation. Scientific reports, 8(1), 1-13.

Stumpf, A., Michéa, D., \& Malet, J. P. (2018). Improved co-registration of Sentinel-2 and Landsat-8 imagery for earth surface motion measurements. Remote Sensing, 10(2), 160. 
Tansock, J., Bancroft, D., Butler, J., Cao, C., Datla, R., Hansen, S., ... \& Murdock, T. (2015). Guidelines for radiometric calibration of electro-optical instruments for remote sensing.

Thome, K. J., Helder, D. L., Aaron, D., \& Dewald, J. D. (2004). Landsat-5 TM and Landsat7 ETM+ absolute radiometric calibration using the reflectance-based method. IEEE Transactions on geoscience and remote sensing, 42(12), 27772785.

Uprety, S., Cao, C., Blonski, S., Shao, X., \& Padula, F. (2013). Tracking On-orbit Radiometric Stability and Accuracy of Suomi NPP VIIRS Using Extended Low Latitude SNOs.

USGS, (2016). Landsat 8 (L8) data users handbook. Department of the Interior US Geological Survey, LSDS-1574.

USGS, Landsat 8 Maneuvers. https://www.usgs.gov/land-resources/nli/landsat/landsat8-maneuvers (Accecido el 14 de Junio de 2020)

USGS, Using the USGS Landsat Level-1 Data Product. https://www.usgs.gov/landresources/nli/landsat/using-usgs-landsat-level-1-data-product (Accecido el 18 de Junio de 2020a)

Zou, C. Z., Goldberg, M. D., Cheng, Z., Grody, N. C., Sullivan, J. T., Cao, C., \& Tarpley, D. (2006). Recalibration of microwave sounding unit for climate studies using simultaneous nadir overpasses.Journal of Geophysical Research: Atmospheres, 111(D19). 


\section{CAPÍTULO 3. ANÁLISIS RADIOMÉTRICO DE LA INFLUENCIA DE LA RSRS EN LAS BANDAS NIR DE SENTINEL 2 A/B MEDIANTE ÁREAS HOMOGÉNEAS.}

\subsection{Introducción}

La RSRs es una de las características más importantes de los sensores ópticos a bordo de los satélites de EO (Gonsamo \& Chen, 2012). La actual variedad de sensores con diferentes RSRs para la misma región del espectro electromagnético, tiene un impacto en la precisión de la calibración y la interoperabilidad de los datos (Chander et al., 2012). Los datos obtenidos sobre el mismo target por sensores con bandas en la misma región espectral, pero con diferentes RSRs, pueden mostrar discrepancias en los valores medidos (Chander et al., 2012; Liang, 2005). Las metodologías de cross-calibration permiten conocer el nivel de incertidumbre de estas discrepancias.

La metodología del SNOs es una de las técnicas más utilizadas por la comunidad científica para la cross-calibration entre dos o más sensores (Chander et al., 2013; Chu \& Dodd, 2019; Uprety et al., 2013; Barrientos et al., 2016; Xu et al., 2014). Esta metodología fue creada y aplicada inicialmente por NOAA-NESDIS para la calibración de los satélites meteorológicos (Cao et al., 2004). La metodología de SNOs se basa en la comparación directa entre satélites (Tansock et al., 2015; Karlsson \& Johansson, 2014). Los sensores que llevan a bordo los satélites observan la misma área de la superficie terrestre casi simultáneamente. Esto permite ver el mismo objetivo en condiciones idénticas de iluminación, visión del satélite, BRDF y efecto atmosférico, reduciendo los posibles errores introducidos por estos factores y mejorando así la incertidumbre de la comparación entre los sensores (Tansock et al., 2015; Uprety et al., 2013; Karlsson \& Johansson, 2014).

Los factores que más influyen en la cross-calibration entre sensores son los relacionados con la RSRs y las características intrínsecas de la escena, como la iluminación, la atmósfera y la superficie. La eliminación o mitigación de cualquiera de estos factores permite mejorar los procesos de calibración cruzada y la interoperabilidad de los datos. El uso de SBAF permite mejorar los resultados en la inter-calibración de los sensores mitigando los efectos de las diferencias de las RSRs entre bandas (Chander et al., 2012; Farhad et al., 2020). Los SBAF no sólo tienen en cuenta la RSRs de los sensores, sino también el perfil espectral de la superficie del blanco (Chander et al., 2012).

En el capítulo 2, se expuso una metodología global de SNOs a partir de áreas homogéneas para la cross-calibration de S2A-MSI y S2B-MSI con L8-OLI. Estableciendo 
un umbral de 30 minutos para el intervalo de tiempo de SNO, un umbral de nubosidad del $5 \%$ en las escenas, una zona de estudio de SNO delimitada, así como la inspección y posterior eliminación de los posibles outliers, se localizaron las áreas homogéneas en las que se realizó la comparación entre los pares de bandas del mismo rango espectral de los sensores. Los resultados mostraron un elevado valor de $\mathrm{R}^{2}$ entre las bandas con una RSRs similar, excepto la banda NIR de L8 y la banda NIR ancha de S2 A/B respectivamente, donde el valor fue menor.

En vista de la influencia de la RSRs en los procesos de cross-calibration, se ha adaptado la metodología global de SNOs basada en áreas homogéneas desarrollada en el capítulo 2 para la cross-calibration de S2A-MSI y S2B-MSI con L8-OLI, centrándonos específicamente en el estudio de las bandas NIR 8 y 8 A de cada sensor MSI de S2 A/B. Este par de bandas NIR, presentan grandes diferencias en su RSRs, provocando una mayor incertidumbre en su cross-calibración como se observa en los resultados obtenidos en el anterior capítulo. Aprovechando la ventaja operacional de tener dos bandas en el mismo rango espectral pero diferente RSR en cada sensor S2, es posible establecer el intervalo de tiempo de SNO entre las bandas NIR de cada satélite igual a 0 segundos, lo que se puede definir como SNO ideal (Figura 44). Estableciendo el intervalo de tiempo de los SNOs igual a 0 segundos, los factores relacionados con la escena como la iluminación y los efectos atmosféricos, son eliminados en la inter-calibración. Este intervalo de tiempo reduce la incertidumbre causada por estos factores, dejando principalmente sólo la influencia relativa a la RSRs y a la superficie de comparación, pudiéndose evaluar empíricamente el efecto de los mismos.

Con este estudio empírico, se pretende medir la influencia de las RSRs y de la superficie de destino en la reflectancia TOA entre las bandas NIR 8 y 8 A de cada satélite S2A y S2B. Estas bandas NIR son de especial importancia como consecuencia de su constante aplicación en diferentes productos de EO, especialmente los relacionados con agricultura y específicamente con índices de vegetación como el Normalized Difference Vegetation Index (NDVI) (D'Odorico et al., 2013; Lange et al., 2017; Wu et al., 2018). Conocer la incertidumbre de forma específica e independiente provocada por la RSRs y la superficie a estudio permite mejorar la comparativa entre las series temporales de datos de evolución de cultivos, bosques, vegetación o incendios, en los que estas bandas son un pilar fundamental. 


\section{SNO IDEAL}

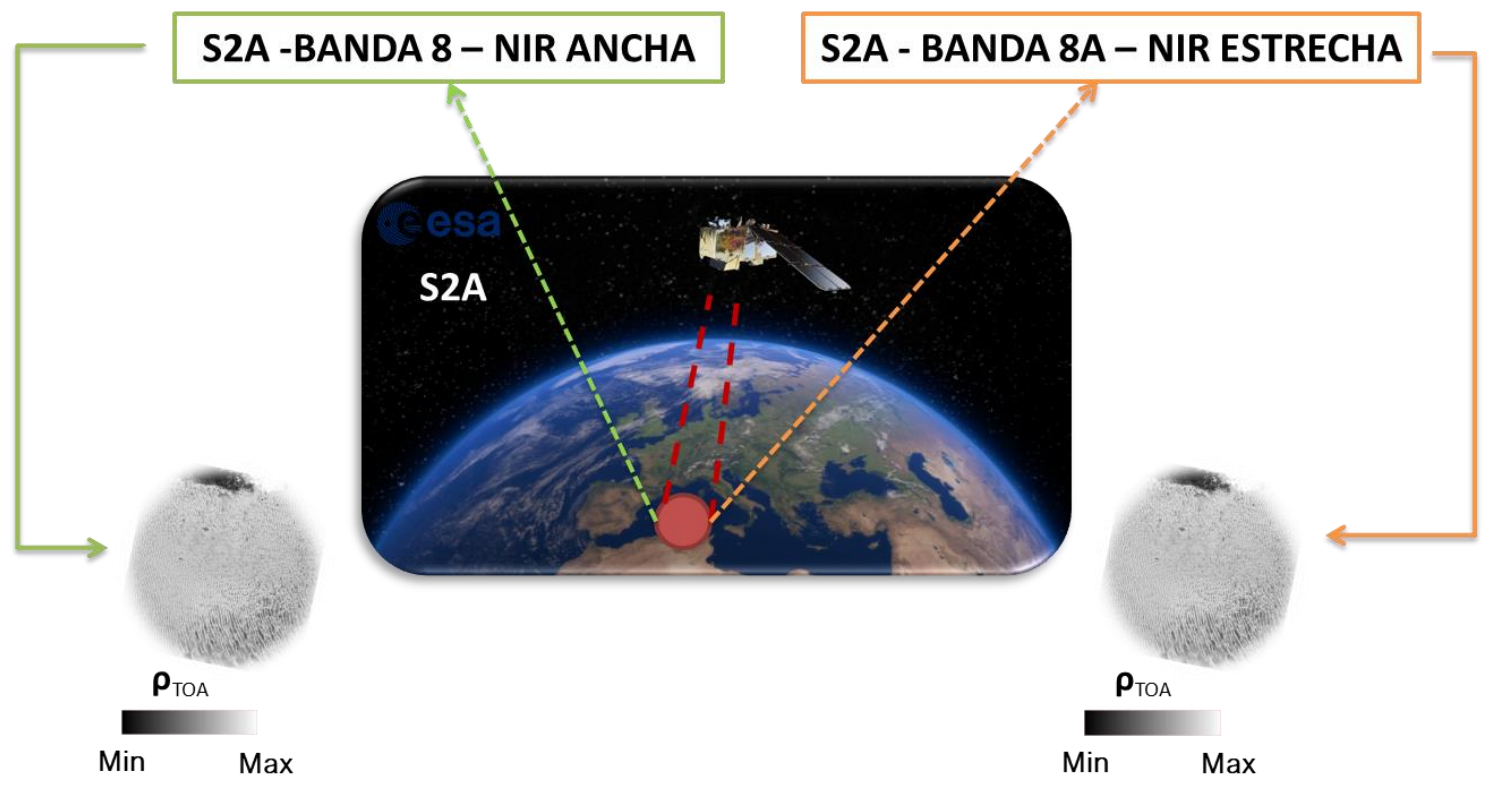

\section{O SEGUNDOS EN LA ADQUISICIÓN ENTRE LAS BANDAS 8 Y 8 A}

Figura 44. Ejemplo de SNO ideal para S2A-MSI.

\subsection{Materiales y métodos}

Para el análisis de la RSRs de las bandas NIR 8 y 8 A de S2 A/B, se hizo uso del mismo data set utilizado en el capítulo 2, por lo tanto, se utilizó el mismo rango temporal de estudio desde el 04-07-2015 hasta 05-11-2019. También se utilizaron las áreas SNOs calculadas en el anterior capítulo para el cálculo de las zonas homogéneas. La utilización del mismo data set temporal y geográfico permitió realizar una mejor comparativa entre los resultados obtenidos entre las bandas NIR de S2 con una SNO ideal y los resultados obtenidos entre sensores diferentes con un margen de tiempo límite de 30 minutos.

\subsubsection{Bandas NIR de Sentinel A/B}

A pesar de tener el mismo sensor MSI, S2A y S2B presentan diferencias en sus RSRs. Como se ha comentado en esta tesis doctoral, dos instrumentos fabricados con el mismo proceso, no son completamente idénticos (Chander et al., 2013a; Chen et al., 2018). Estas diferencias son fácilmente visibles en la comparativa de las RSRs de cada satélite de la misión S2 (ESA, 2018). En la Figura 45 se muestra la comparativa de las RSRs de S2 A/B para las bandas del rango espectral VNIR. Para este estudio, se han utilizado de manera independiente, los pares de bandas NIR ancha (8) y NIR estrecha (8A) de S2 A y S2B para su estudio (Figura 46). 
S2A \& S2B MSI Spectral Response Average - VNIR

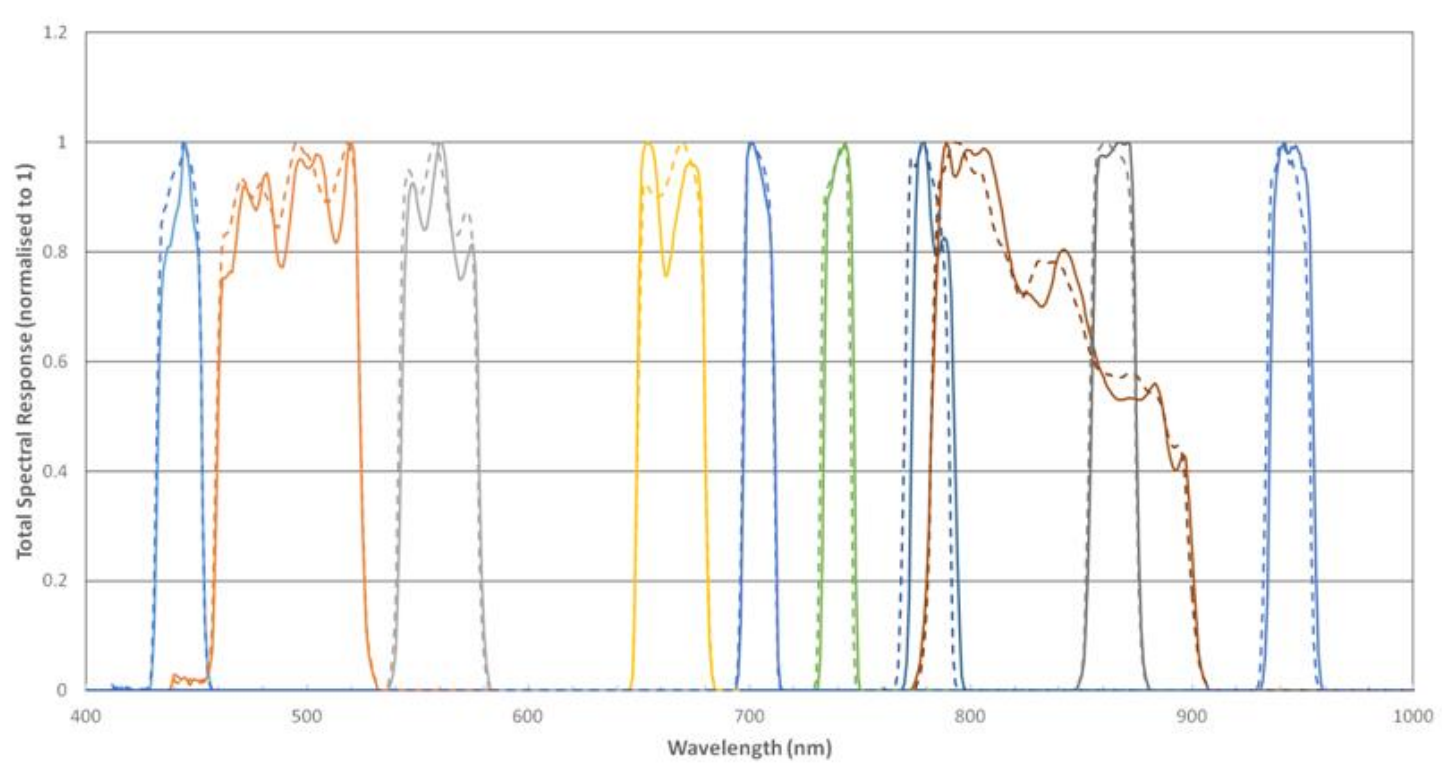

Figura 45. Comparativa de las RSRs de las bandas VNIR de S2A-MSI y S2B-MSI. Fuente: ESA (2018). 


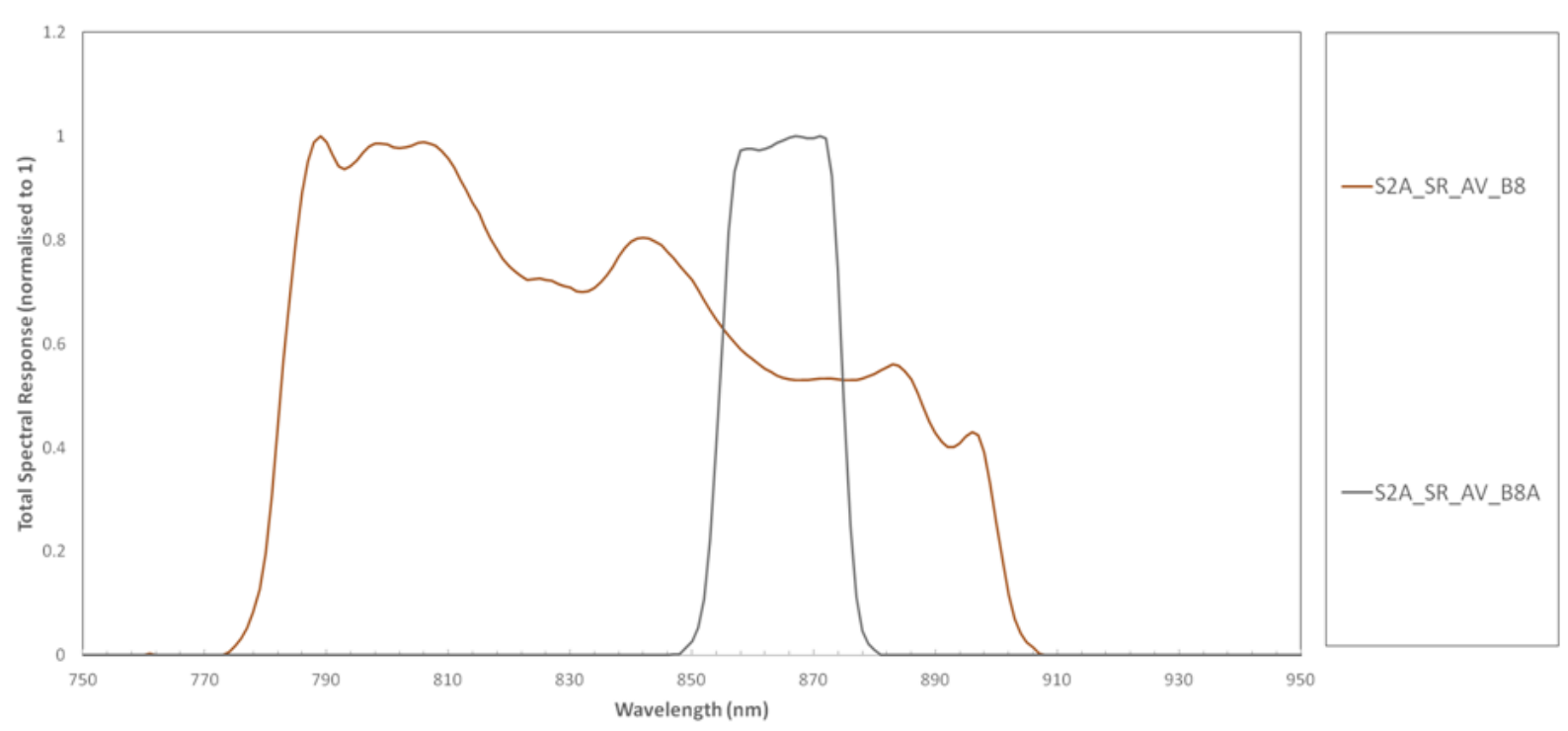

S2B MSI Spectral Response Average - BANDS 8 and 8 A

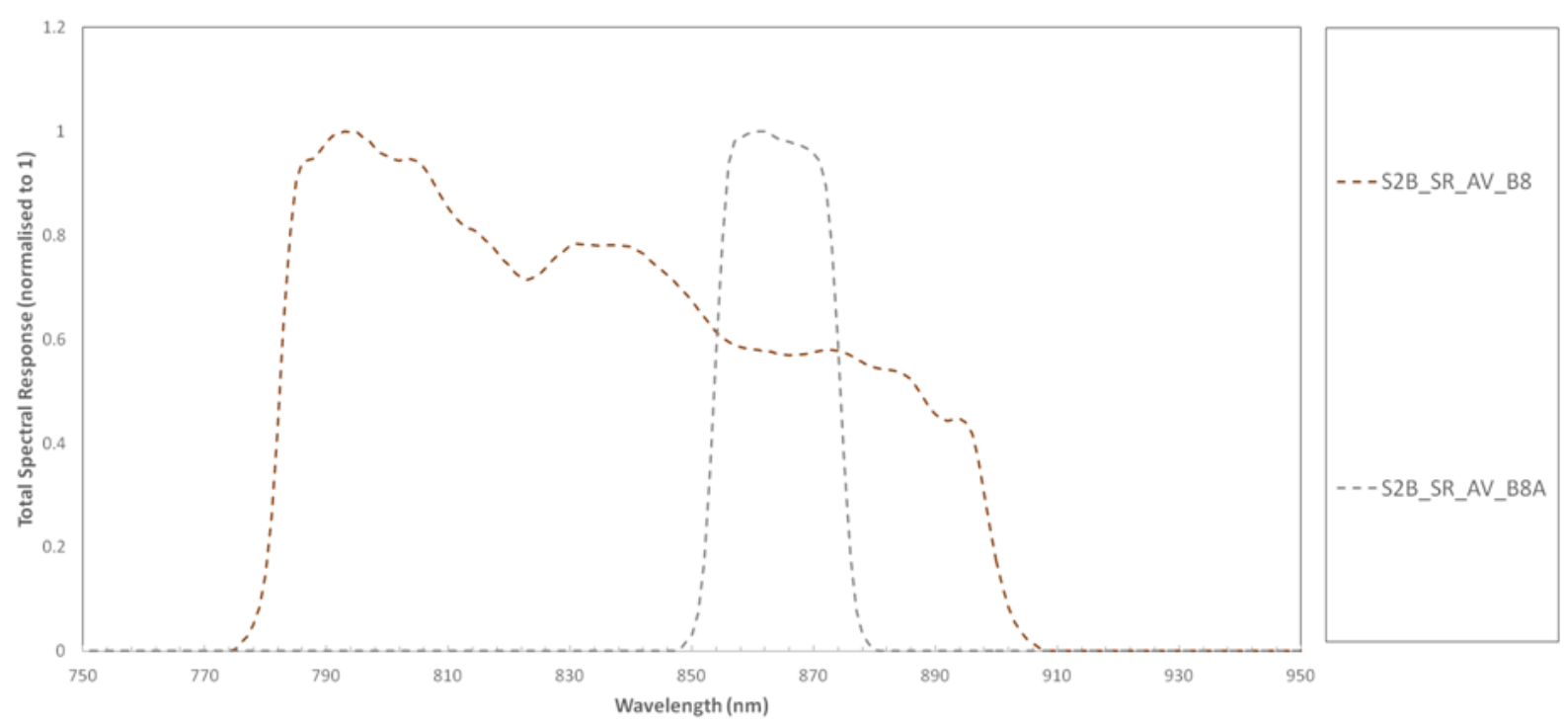

Figura 46. Comparativa de las RSRs de los pares de bandas NIR ancha (8) y NIR estrecha (8A) de S2A-MSI y S2B-MSI utilizados para el análisis. Fuente: Adaptada de ESA (2018).

\subsubsection{SNOs Sentinel A/B}

Se utilizaron las SNOs definidas en la comparativa de L8 con respecto a S2A y S2B del capítulo anterior. Esta definición de SNOs permite fijar el punto nadir de las mismas, así como delimitar perfectamente el área del centroide para el cálculo y posterior análisis de las áreas homogéneas. En la Figura 47 se muestra la localización global de las SNOs utilizadas, estando las áreas sobre-escaladas para una mejor visualización. 

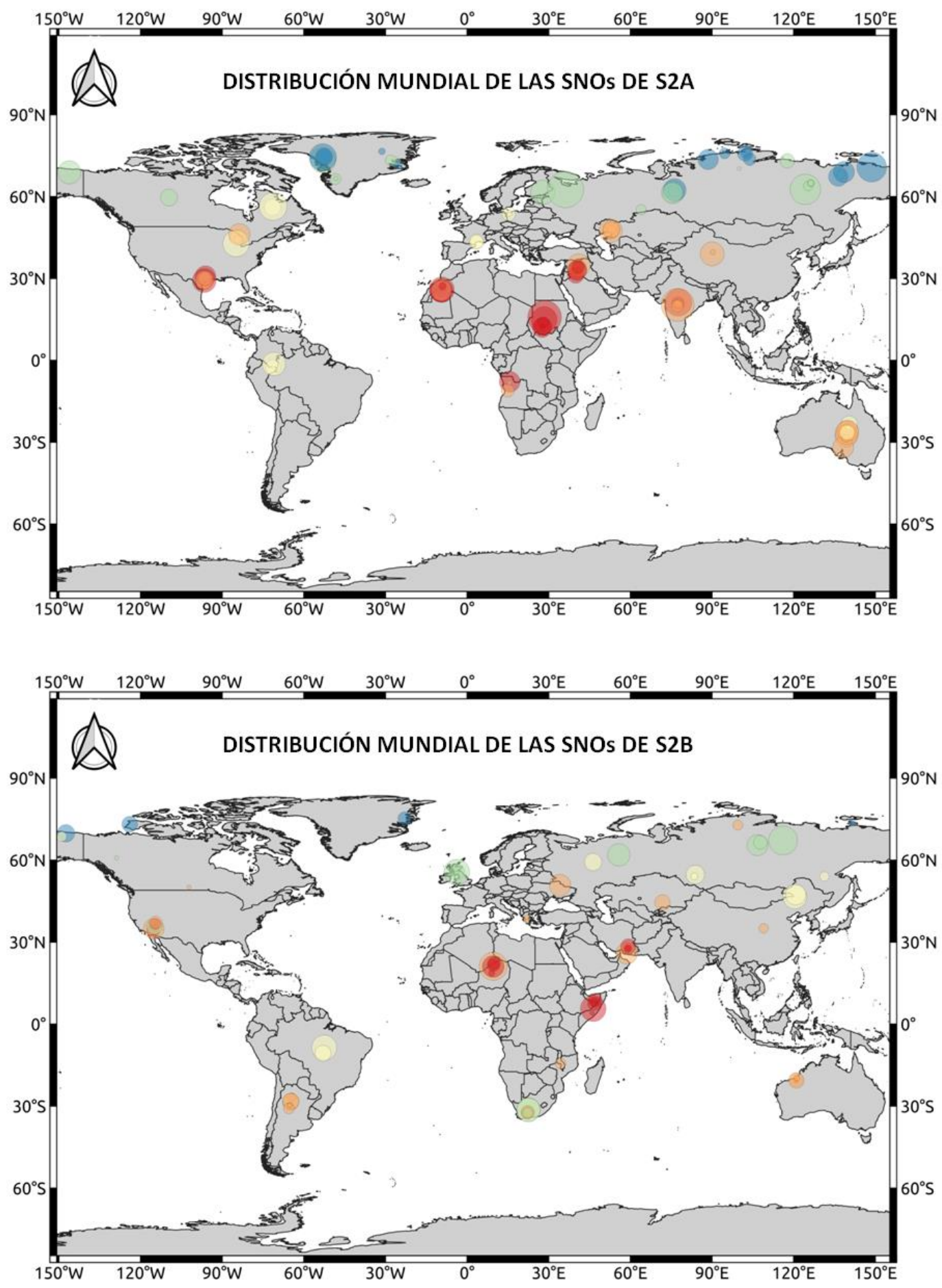

Figura 47. Distribución global de las SNOs ideales, utilizadas para la comparativa de cada par de bandas NIR de S2A y S2B.

\subsubsection{Uniformidad espacial y áreas homogéneas}

Para poder determinar las zonas homogéneas a comparar dentro del área de estudio definida previamente por las SNOs, se utilizó como métrica de homogeneidad el CV espacial. Al igual que en el capítulo anterior, no tenía sentido el estudio temporal al realizarse la adquisición en condiciones de completa simultaneidad y sobre una única escena. 
La elección de cuál de las dos bandas NIR se debía utilizar para el cálculo del CV espacial fue adoptada en base al criterio de resolución espacial de las mismas. La banda 8 tiene una resolución espacial de 10 metros, mientras que la resolución espacial de la banda $8 \mathrm{~A}$ es de 20 metros. La mayor resolución espacial de la banda 8, permite una mejor caracterización de la uniformidad espacial de la imagen, por lo tanto, fue la banda seleccionada para la generación del CV espacial.

El cálculo de las áreas homogéneas se realizó siguiendo la misma metodología del capítulo anterior. Se estableció un umbral a partir del valor de CV correspondiente al percentil 1 de la muestra. Posteriormente, se procedió a binarizar las imágenes para diferenciar las zonas seleccionadas como las más homogéneas de las que no, para finalmente aplicar el operador erosión (Piper, 1985; Liang et al., 1989) y el operador dilatación (Piper, 1985; Liang et al., 1989) para eliminar los píxeles aislados, así como, para que los grupos de píxeles afectados por el operador erosión recuperen parcialmente su tamaño inicial. Una vez realizadas todas estas operaciones se procede a vectorizar las áreas homogéneas calculadas. Este proceso se realizó para cada SNO localizada y banda 8 NIR de S2A y S2B, creándose un conjunto de áreas homogéneas para cada SNO.

Una vez obtenido el vector de áreas homogéneas para cada SNO y banda 8 NIR de S2A y S2B, se realiza la conversión de los valores de DN a valores de reflectancia TOA teniendo en cuenta la influencia del ángulo solar de la escena (ESA, 2020a).

\subsubsection{Comparativa entre sensores, análisis de datos y eliminación de outliers.}

Los valores de reflectancia TOA obtenidos sobre las áreas homogéneas para cada par de bandas NIR 8-8A de S2A y S2B, se compararon mediante una regresión lineal.

Debido a que las bandas que se comparan están a bordo del mismo sensor, el tiempo de SNO es el ideal, es decir 0 segundos. Este factor, facilita la detección de los outliers de una forma más ágil que la expuesta en el capítulo 2. En ese caso, era necesaria la realización de una inspección visual debido a la diferencia de 30 minutos establecida entre adquisiciones SNOs. Esa diferencia de tiempo provocaba que algunas áreas homogéneas de una de las bandas a estudio se encontrasen afectadas por nubosidad, zonas de sombra, áreas nubladas, etc., mientras que las áreas de la otra banda no.

En este caso particular de SNO ideales, al ser la adquisición puramente simultánea, la comparativa sobre nubes, cuerpos de agua o zonas de sombra, se realiza en ambas bandas 8 y $8 \mathrm{~A}$ a la vez, sin que se produzcan cambios o desplazamientos de estos fenómenos. Esta circunstancia permitió la automatización del proceso de eliminación de outliers, eliminando únicamente los píxeles afectados por la BRDF, ya que estos píxeles presentaban valores de reflectancia TOA superiores a 1 . Estableciendo un umbral de reflectancia TOA de 1 , se pudo eliminar de forma automática los posibles outliers de la comparativa (Figura 48). 


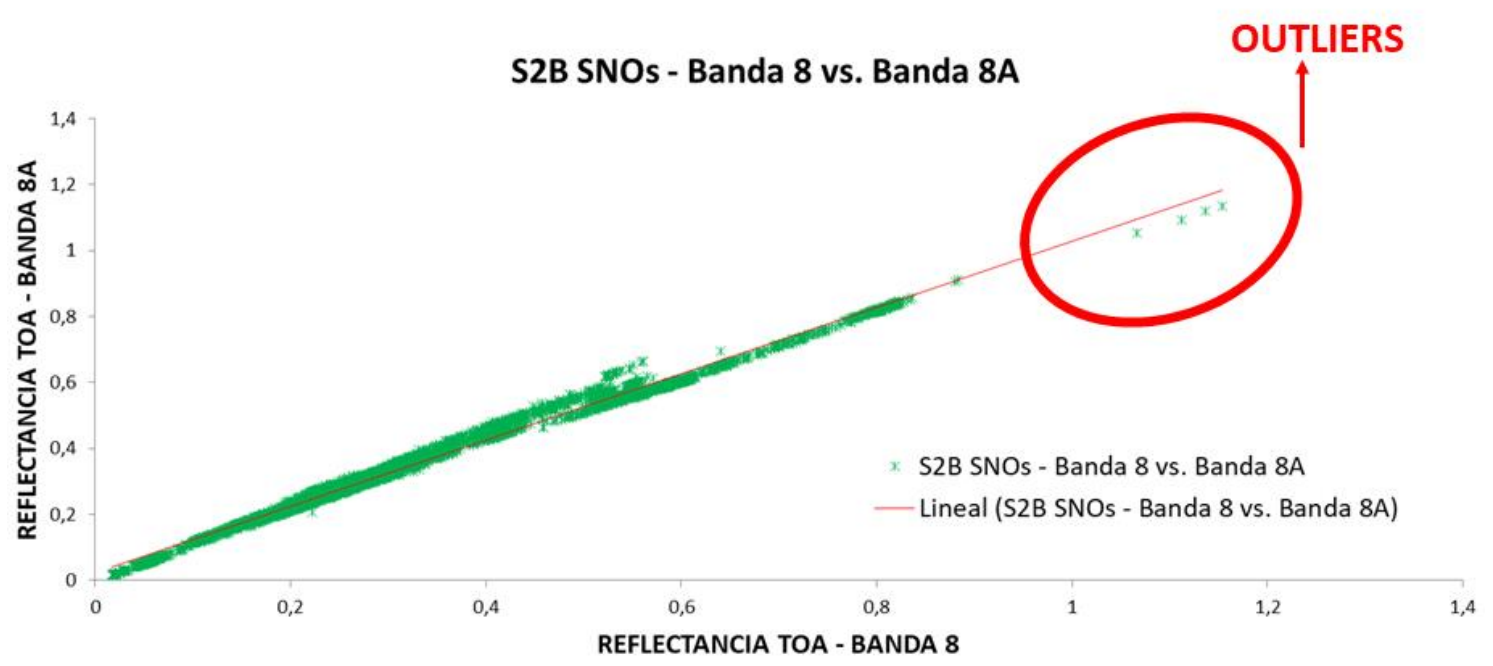

Figura 48. Eliminación de outliers afectados por la BRDF con valores de reflectancia TOA superiores a 1.

\subsubsection{Análisis de las clases de superficies de suelo utilizadas.}

Debido a la naturaleza global de la metodología, fue necesario realizar un análisis de las clases de suelo que habían participado en el mismo. Para ello, se calculó la distribución de clases de suelo utilizadas en la comparativa de cada par de bandas NIR 8-8A a partir de la aplicación del land cover CGLS-LC100 (Buchhorn et al., 2019) sobre las áreas homogéneas. Esto no solo permitió conocer la distribución de las clases de superficie utilizadas y su ponderación, sino también el comportamiento de la metodología en diferentes superficies con características espectrales muy dispares.

Para una mejor comprensión del método, en la Figura 49 se presenta un esquema general del flujo de trabajo de la metodología de las SNOs ideal, para las bandas 8-8A de S2A y S2B mediante zonas homogéneas. 


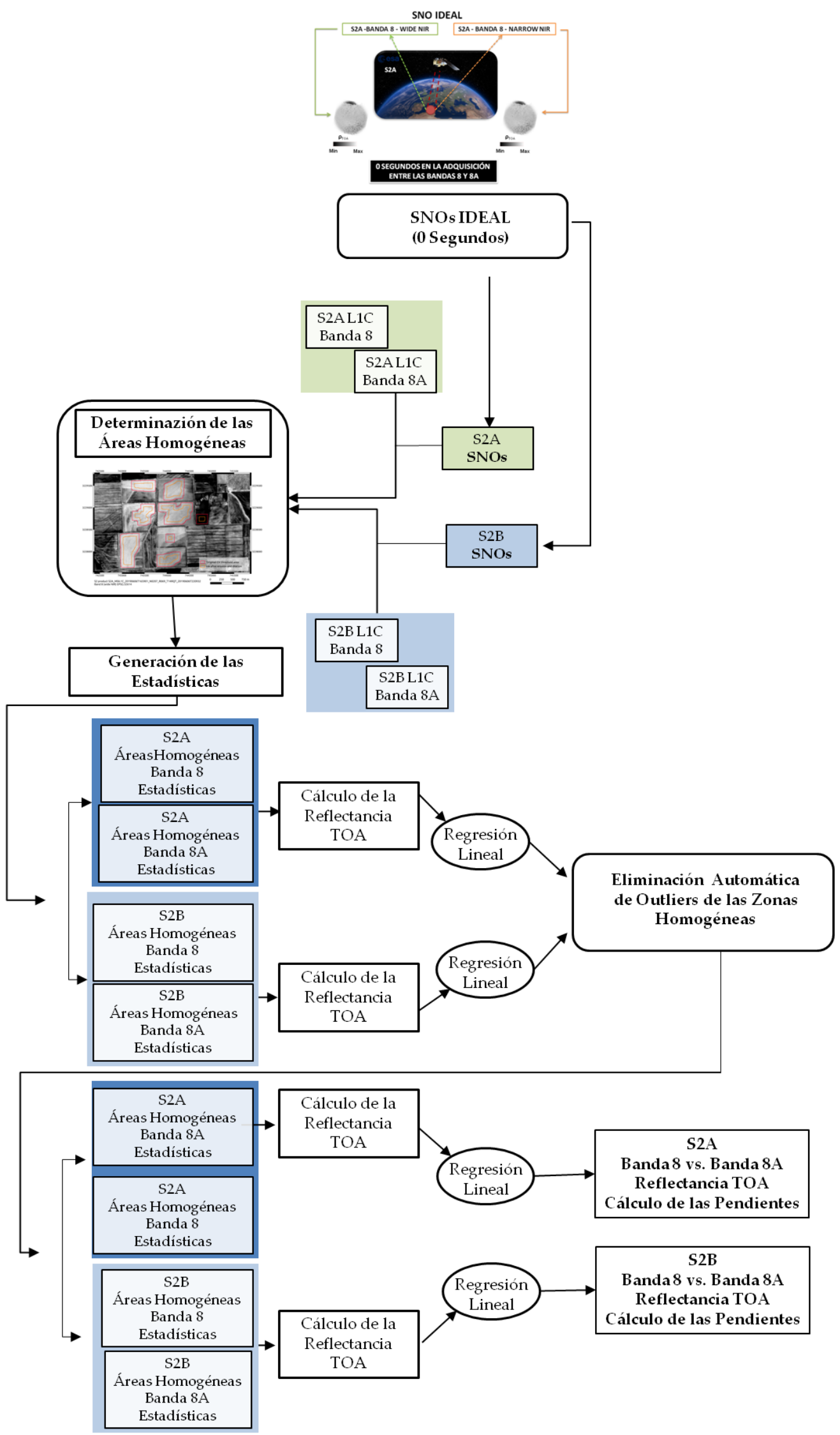

Figura 49. Flujo de trabajo de la metodología de SNOs ideales, utilizada para la comparativa de cada par de bandas NIR 8-8A de S2 A/B mediante áreas homogéneas. 


\subsection{Resultados}

\subsubsection{Comparativa de los valores de reflectancia TOA.}

Para cada par de bandas NIR 8-8A de S2A y S2B se realizó una regresión lineal de los valores de reflectancia TOA de las áreas homogéneas con los outliers previamente eliminados. También se calcularon las incertidumbres de la comparativa para establecer un amplio intervalo de confianza de la medida, para ello se fijó un rango de $\pm 3 \sigma$ de los residuos.

En la Tabla 12 y Tabla 13 se muestran los resultados de la regresión lineal de cada par de bandas NIR 8-8A de S2A y S2B, mientras que la Figura 50 y Figura 51 muestran los gráficos de la comparativa de las regresiones lineales. Cada punto es un área homogénea diferente y la línea roja es la regresión lineal.

Los valores obtenidos en la Tabla 12 y Tabla 13 muestran un $\mathrm{R}^{2}$ muy elevado, prácticamente cercano a 1. Para el caso del valor de offset de la comparativa en ambos satélites, las bandas presentan valores cercanos a 0 .

Tabla 12. Resultados de la regresión lineal de la comparativa de valores de reflectancia TOA para cada par de bandas NIR 8-8A de S2A.

\begin{tabular}{cccc}
\hline $\begin{array}{c}\text { Banda } \\
\text { S2A }\end{array}$ & Pendiente & Intersección & $\mathbf{R}^{\mathbf{2}}$ \\
\hline 8 vs. 8A & 1.0279 & 0.0171 & 0.9936 \\
\hline
\end{tabular}

Tabla 13. Resultados de la regresión lineal de la comparativa de valores de reflectancia TOA para cada par de bandas NIR 8-8A de S2B.

\begin{tabular}{cccc}
\hline $\begin{array}{c}\text { Banda } \\
\text { S2B }\end{array}$ & Pendiente & Intersección & $\mathbf{R}^{2}$ \\
\hline $\mathbf{8}$ vs. 8A & 1.0068 & 0.0228 & 0.9923 \\
\hline
\end{tabular}




\section{S2A SNOs - Banda 8 vs. Banda 8A}

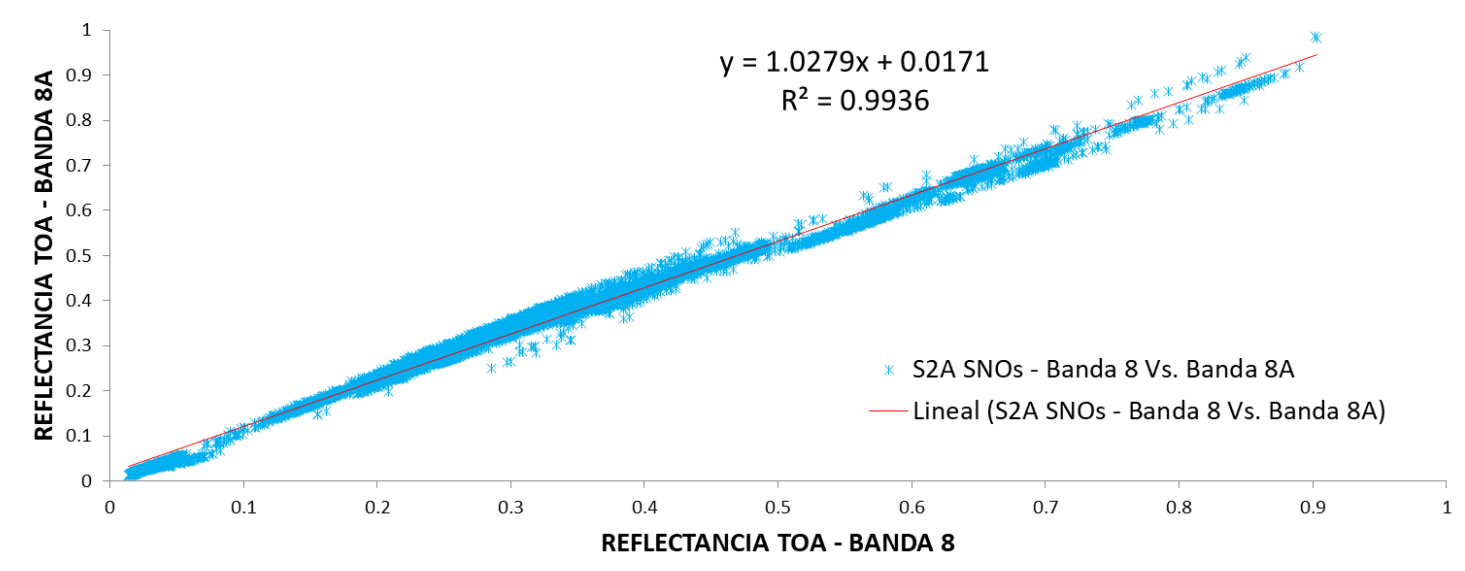

Figura 50. Gráfica de la regresión lineal para el par de bandas NIR 8-8A a estudio de S2A, de los valores de reflectancia TOA calculados sobre zonas homogéneas.

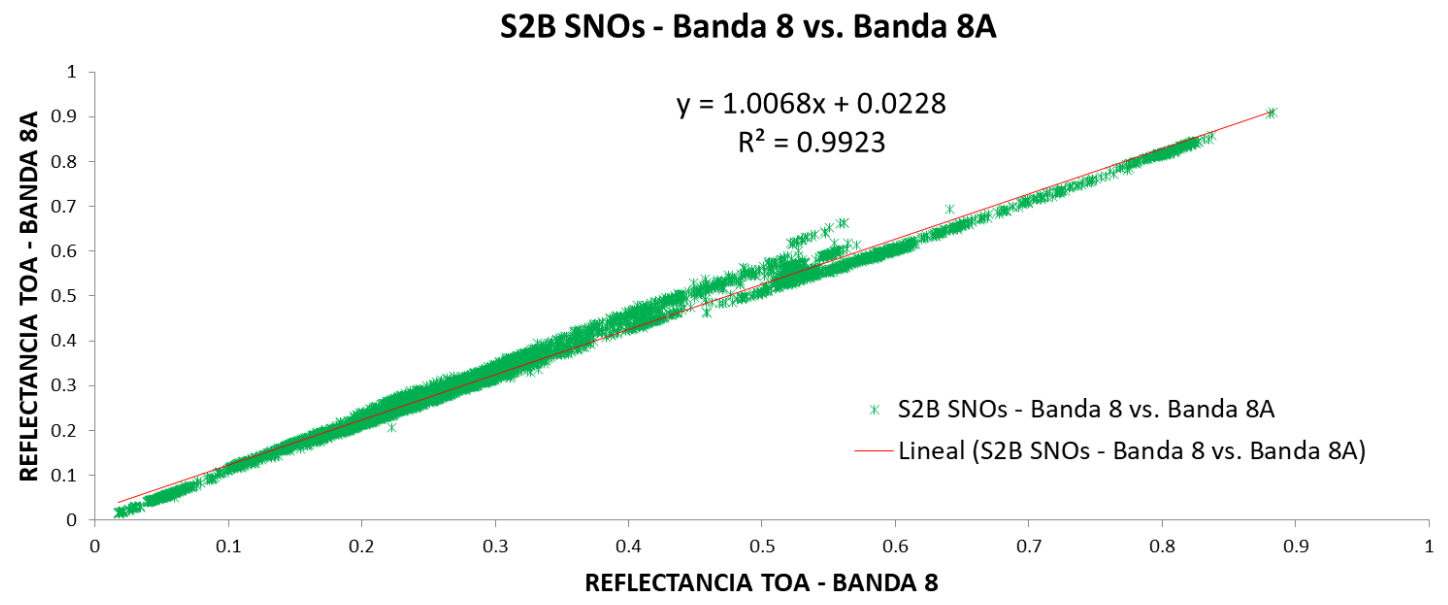

Figura 51. Gráfica de la regresión lineal para el par de bandas NIR 8-8A a estudio de S2B, de los valores de reflectancia TOA calculados sobre zonas homogéneas.

Este proceso de regresión lineal se vuelve a repetir, pero eliminando el grado de libertad de la ordenada en el origen forzándolo a cero, debido a los resultados obtenidos y a que se puede asumir que la reflectancia TOA de los sensores en ausencia de señal será 0 . En la Tabla 14 y Tabla 15, así como en la Figura 52 y Figura 53, se pueden encontrar los resultados de esta nueva regresión lineal forzando a cero el valor de intersección. Cada punto es un área homogénea diferente y la línea roja es la regresión lineal forzada a 0.

Tabla 14. Resultados de la regresión lineal con el valor de intersección forzado a cero, de la comparativa de valores de reflectancia TOA para el par de bandas NIR 8-8A de S2A.

\begin{tabular}{ccc}
\hline $\begin{array}{c}\text { Banda } \\
\text { S2A }\end{array}$ & Pendiente & $\mathbf{R}^{\mathbf{2}}$ \\
\hline $\mathbf{8}$ vs. 8A & 1.0676 & 0.9982 \\
\hline
\end{tabular}


Tabla 15. Resultados de la regresión lineal con el valor de intersección forzado a cero, de la comparativa de valores de reflectancia TOA para el par de bandas NIR 8-8A de S2B.

\begin{tabular}{ccc}
\hline $\begin{array}{c}\text { Banda } \\
\text { S2B }\end{array}$ & Pendiente & $\mathbf{R}^{\mathbf{2}}$ \\
\hline $\mathbf{8}$ vs. 8A & 1.0589 & 0.9978 \\
\hline
\end{tabular}

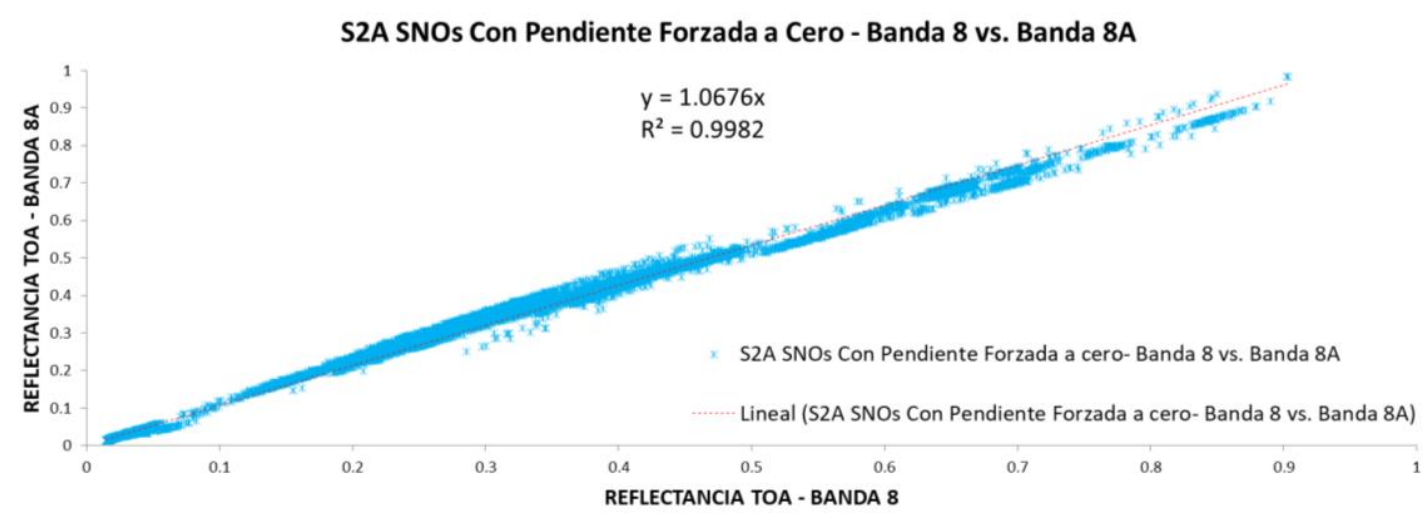

Figura 52. Regresión lineal para el par de bandas NIR 8-8A de S2A con el valor de la intersección forzada a 0.

S2B SNOs Con Pendiente Forzada a Cero - Banda 8 vs. Banda 8A

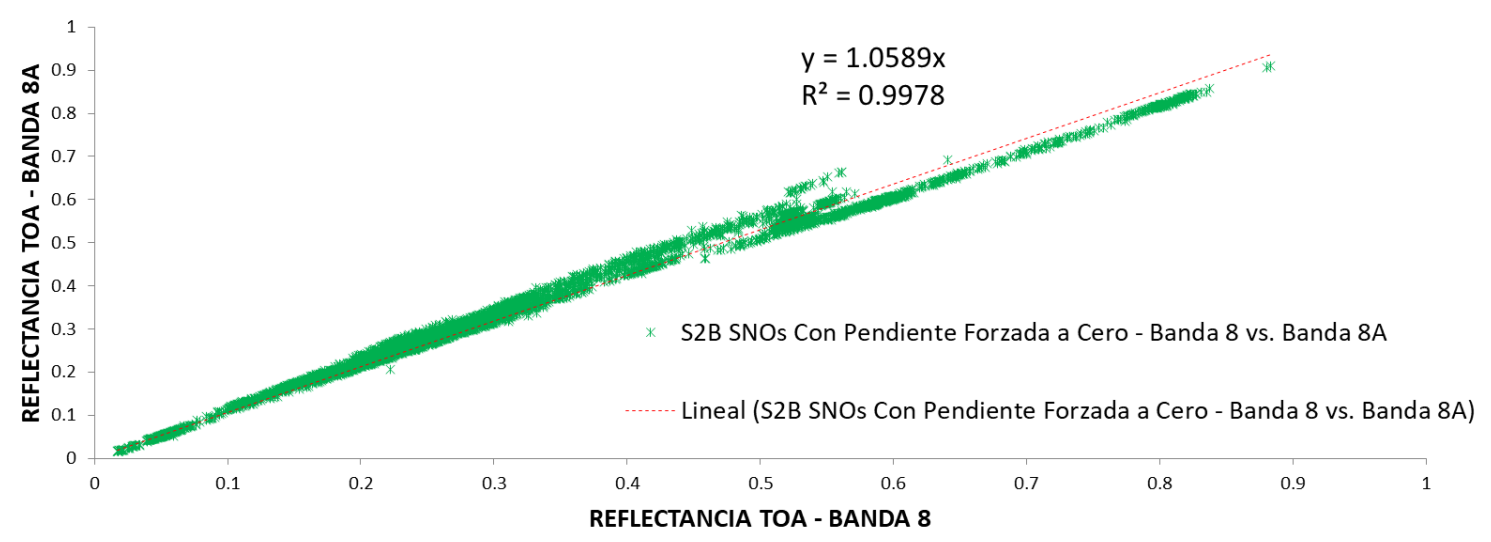

Figura 53. Regresión lineal para el par de bandas NIR 8-8A de S2B con el valor de la intersección forzada a 0.

\subsubsection{Análisis de las clases de superficies.}

Se consideró conveniente el estudio de las diferentes clases de superficies implicadas en el análisis. Esta información permite conocer la distribución y posible ponderación de cada clase de superficie. Para ello se utilizó el land cover CGLS-LC100 (Buchhorn et al., 2019) y el vector de áreas homogéneas de cada par de bandas y sensores S2. En la Tabla 7 se muestran los diferentes tipos de clases del CGLS-LC100 y el identificador asignado, mientras que en la Figura 54 y Figura 55 se muestra la distribución de las clases del CGLSLC100 sobre las áreas homogéneas para cada par de bandas NIR 8-8A de los sensores S2 A/B. 


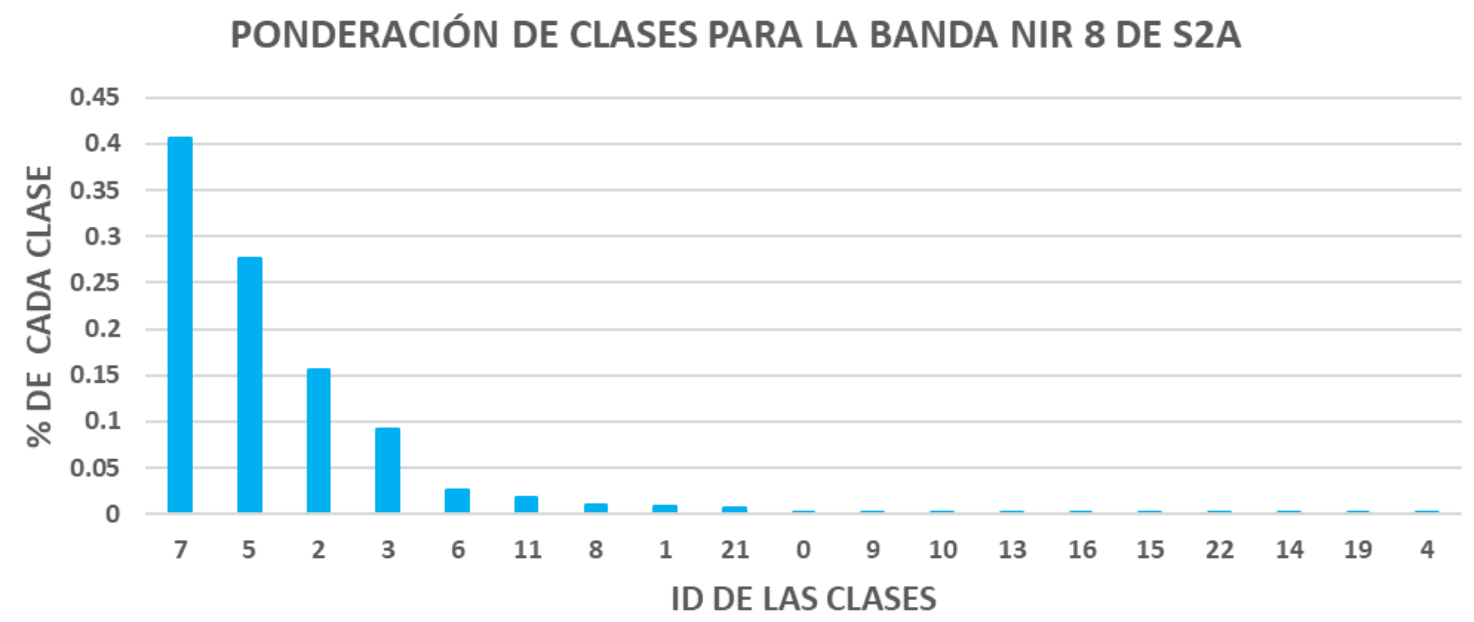

Figura 54. Distribución de clases del CGLS-LC100 sobre las áreas homogéneas de la banda 8 NIR de S2 A.

PONDERACIÓN DE CLASES PARA LA BANDA NIR 8 DE S2B

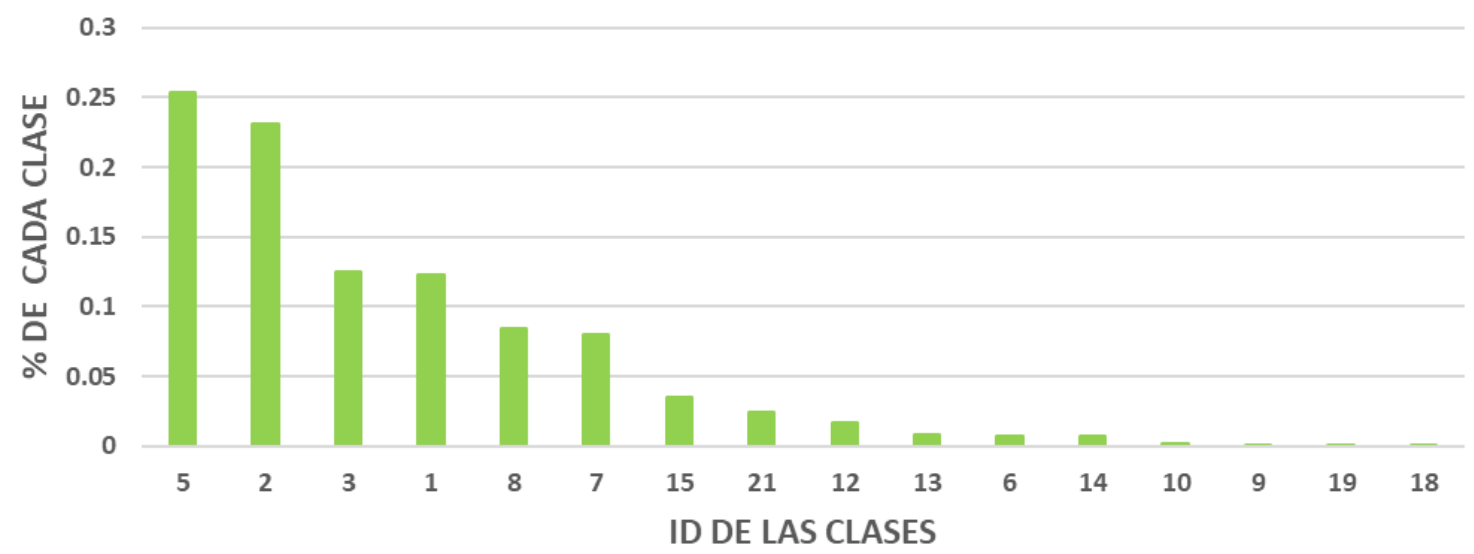

Figura 55. Distribución de clases del CGLS-LC100 sobre las áreas homogéneas de la banda 8 NIR de S2 B.

\subsection{Discusión}

En este capítulo se ha presentado una metodología empírica de SNOs ideal entre las bandas NIR 8-8A de S2A y S2B sobre áreas homogéneas sin necesidad de realizar ningún tipo de corrección atmosférica o BRDF. Al realizarse las adquisiciones de ambas bandas NIR de cada satélite S2 en un intervalo de tiempo de 0 segundos, las incertidumbres asociadas a efectos atmosféricos, de BRDF y de iluminación de la escena quedan eliminados, permitiendo observar únicamente los efectos derivados de las diferencias en las RSRs entre bandas y superficies espectrales utilizadas.

A diferencia de la utilización de los PICS como target de cross-calibration, los cuales presentan una localización fija y valores de reflectancia muy elevados (Neigh et al., 2015; Mishra et al., 2014; Chander et al., 2013; Chander et al., 2010), la gran variedad de 
localizaciones utilizadas en este estudio permite cubrir prácticamente todo el rango de reflectancias TOA en la comparativa entre bandas.

En esta metodología, se ha implementado un sencillo método de eliminación de outliers a partir del establecimiento de un umbral de reflectancia TOA de los efectos de la BRDF en algunas de las zonas homogéneas. La simultaneidad de las adquisiciones facilita que ambas bandas observen siempre la misma zona en las mismas condiciones. En esta adaptación de la metodología para las bandas NIR 8-8A de S2 A/B, la nubosidad, las sombras o los cuerpos de agua no son factores distorsionadores de la comparativa, al estar presentes en ambas bandas en condiciones idénticas de observación. En cambio, en la metodología de SNOs presentada en el capítulo 2 entre S2 A/B y L8, el intervalo de tiempo entre adquisiciones permitía la aparición de nubosidad, sombras u otros factores dinámicos en alguna adquisición, provocando la aparición de outliers en la comparativa entre sensores.

Los resultados obtenidos muestran una gran correlación entra los pares de bandas NIR 8-8A a estudio de S2A-MSI y S2B-MSI (Tabla 14 y Tabla 15). A pesar de la diversidad de superficies utilizadas (Figura 54 y Figura 55) y las diferencias en RSRs de ambas bandas NIR (Figura 46), el $R^{2}$ muestra valores cercanos a 1 , con un valor de $R^{2}$ de 0.9982 en el caso de S2A y 0.9978 en S2B.

De forma general, el desglose de las clases de superficie muestra una amplia variedad y cubre desde desiertos, definidos como clase 5 (suelo desnudo / vegetación escasa), hasta la clase 7, masas de agua permanentes. La distribución de las diferentes clases de superficies utilizadas, difieren para cada satélite S2 (Figura 54 y Figura 55). La clase 7 (masas de agua permanentes) está fuertemente representada en el S2A con un valor aproximado del $40 \%$, no llegando al $10 \%$ en el caso de S2B. A pesar de ello, existe una coincidencia entre ambos satélites en el resto de clases con elevada presencia, como son las clases 5 - suelo desnudo / vegetación escasa, 2 - vegetación cultivada y gestionada, 3 - vegetación herbácea (Figura 54 y Figura 55). Estas diferencias son debidas a la diferente distribución mundial de los SNOs para cada satélite S2 (Figura 47).

Para la validación del método, fue necesario realizar la comparativa con diferentes metodologías y targets. Siguiendo las recomendaciones de Helder et al. (2018), se utilizó la métrica del ratio como comparativa entre metodologías, al eliminar posibles errores sistemáticos dentro de los modelos y proporcionar una comparación por modelo.

Al no existir ningún estudio previo que haya realizado la comparativa entre las bandas NIR de cada satélite S2, la validación del método es realizada con los resultados obtenidos en el informe sobre el estado de la calibración de la misión S2 realizados por el MPC (ESA, 2020b) entre las bandas NIR 5 de L8 y 8 de S2. Al presentar ambas bandas NIR grandes diferencias en sus RSRs, podemos comparar esos resultados con los 
obtenidos en esta metodología. En la Figura 56 y Figura 57 se muestran los resultados y la comparativa entre los métodos evaluados por MPC y la metodología de SNOs ideal.

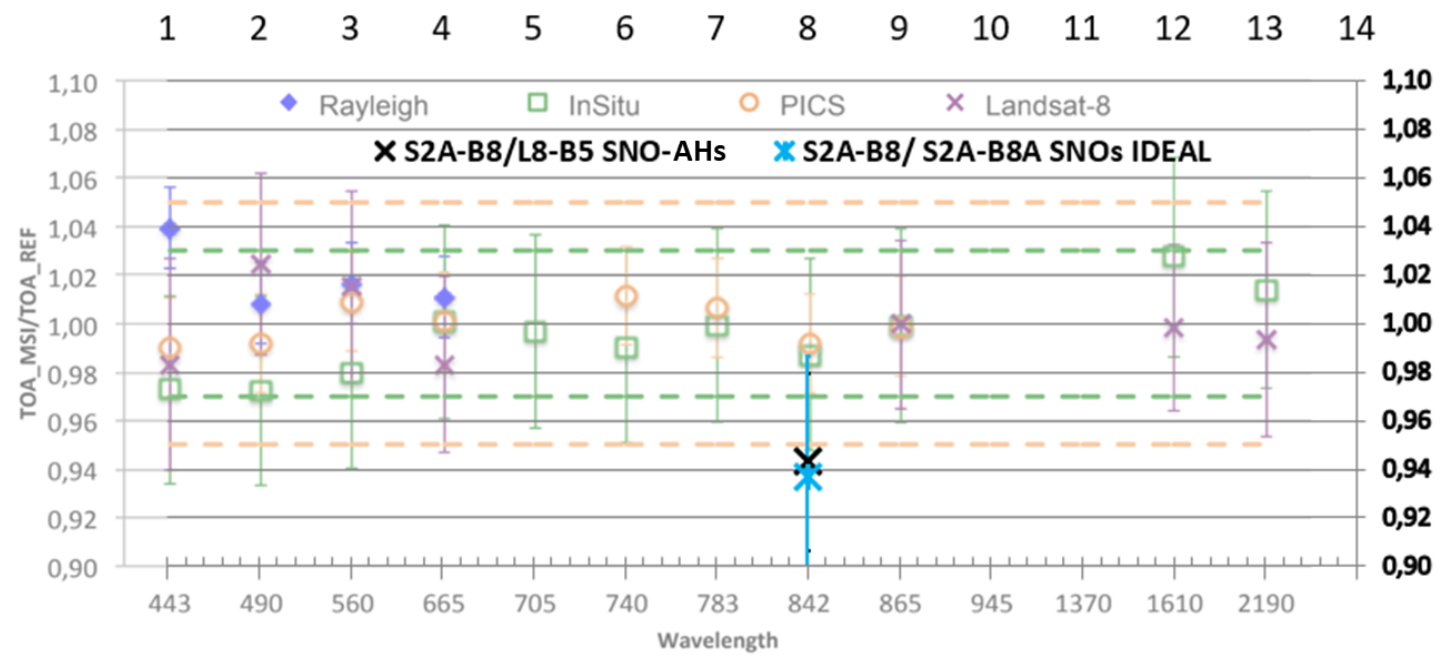

Figura 56. Comparativa de los ratios de las pendientes para los métodos utilizados por el MPC y la metodología de SNOs Ideales mediante áreas homogéneas para S2A, representada por cruces azules sólidas en la gráfica. La metodología de SNOs Ideales muestra en sus resultados un intervalo de confianza de $\pm 3 \sigma$.

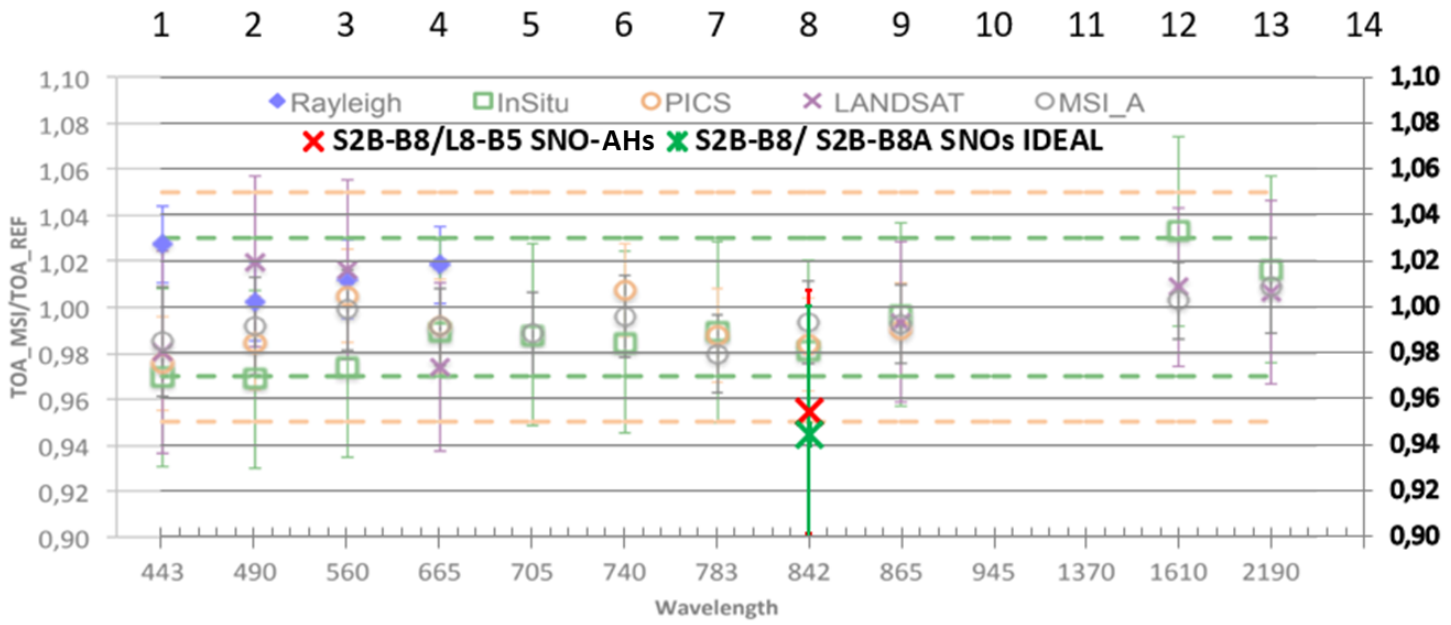

Figura 57. Comparativa de los ratios de las pendientes para los métodos utilizados por el MPC y la metodología de SNOs Ideales mediante áreas homogéneas para S2B, representada por cruces verdes sólidas en la gráfica. La metodología de SNOs Ideales muestra en sus resultados un intervalo de confianza de $\pm 3 \sigma$.

Tanto en la comparativa con otras metodologías como en la comparativa de los resultados de la metodología de SNOs ideales con respecto al método original de SNOs presentado en el capítulo anterior (Figura 56 y Figura 57), los resultados presentan una elevada coincidencia. Este resultado confirma la robustez del método de SNOs mediante áreas homogéneas independientemente de que el intervalo de tiempo de la adquisición 
sea de 0 segundos o de 30 minutos, siempre y cuando los outliers sean eliminados. Se observa una menor incertidumbre en los resultados de los pares de bandas NIR de S2 $A / B$, con una mayor diferencia en su RSRs adquiridos con el método de SNOs Ideales de 0 segundos. Para este método, los valores de $R^{2}$ son de 0.9936 para S2A y de 0.9923 para S2B, mientras que con la metodología de SNOs entre las bandas 8 de S2 A/B y la Banda 5 de $L 8$ los valores de $R^{2}$ son de 0.980 y 0.984 respectivamente.

Los resultados de la metodología de SNOs Ideales con otros métodos, validan y confirman este nuevo enfoque propuesto para la interoperabilidad y armonización de los datos de satélite con bandas del mismo rango espectral, pero con diferente RSRs a bordo del mismo sensor. Especialmente importante es su aplicación para series temporales de datos de NDVI para S2, al duplicarse las posibilidades de datos al facilitarse la comparativa y armonización de los mismos.

\subsection{Conclusiones}

En este capítulo, se ha analizado la influencia de la RSRs para bandas del mismo rango espectral, pero con grandes diferencias en su RSRs. Para ello, se ha adaptado la metodología global de cross-calibration basada en SNOs a partir de áreas homogéneas sobre cualquier tipo de superficie espectral presentada en el capítulo anterior. Haciendo uso de la ventaja que permiten los satélites de la constelación S2 (dos bandas NIR con grandes diferencias en sus RSRs) es posible generar un dataset de SNOs ideales, es decir con tiempo de adquisición de 0 segundos. Esta novedosa metodología presenta ventajas muy importantes al eliminar las incertidumbres derivadas de las condiciones de iluminación de la escena, los efectos de BRDF y efectos atmosféricos, quedando únicamente los efectos relativos a la RSRs y al tipo de superficie analizada. Además, es importante destacar que la perfecta simultaneidad de las SNOs ha permitido automatizar la eliminación de los outliers de manera ágil y sencilla.

La utilización del mismo dataset global del capítulo 2, ha permitido comparar los resultados obtenidos para las bandas NIR de L8 y S2 con grandes diferencias en sus RSRs, con los obtenidos para S2 mediante la metodología de SNOs ideal. Debido a la naturaleza global de los datos, la comparativa se realizó sobre un gran número de usos de suelo lo que garantiza una gran diversidad de reflectancias.

Se registraron uno altos valores de $\mathrm{R}^{2}$ para las bandas $8-8 \mathrm{~A}$ de ambos satélites $\mathrm{S} 2$. La validación de la metodología de las SNOs ideales con otros métodos han confirmado la robustez del método y su posible utilización como técnica para la armonización e interoperabilidad de los datos de diferentes sensores de satélites. Los resultados de esta comparativa muestran valores de ratio y de incertidumbres prácticamente similares entre la metodología de SNOs y SNOs ideales, demostrando que las diferencias de tiempo entre adquisiciones no es un factor influyente cuando los outliers son eliminados y el umbral de tiempo establecido no es muy elevado. 
Esta metodología global de SNOs ideales presentada, debido a su agilidad en la implementación y operatibililidad, puede extenderse para la evaluación de multiples sensores de satélites con bandas espectrales del mismo rango, pero con diferente RSRs.

\subsection{Referencias}

Barrientos, C., Mattar, C., Nakos, T., \& Perez, W. (2016). Radiometric Cross-Calibration of the Chilean Satellite FASat-C Using RapidEye and EO-1 Hyperion Data and a Simultaneous Nadir Overpass Approach. Remote Sensing, 8(7), 612.

Buchhorn, M., Smets, B., Bertels, L., Lesiv, M., Tsendbazar, N. E., Herold, M., \& Fritz, S. (2019). Copernicus Global Land Service: Land Cover 100m: Epoch 2015: Globe. Version V2. 0.2.

Cao, C., Weinreb, M., \& Xu, H. (2004). Predicting simultaneous nadir overpasses among polar-orbiting meteorological satellites for the intersatellite calibration of radiometers. Journal of Atmospheric and Oceanic Technology, 21(4), 537-542.

Chander, G., Haque, M. O., Sampath, A., Brunn, A., Trosset, G., Hoffmann, D., ... \& Anderson, C. (2013a). Radiometric and geometric assessment of data from the RapidEye constellation of satellites. International Journal of Remote Sensing, 34(16), 5905-5925.

Chander, G., Hewison, T. J., Fox, N., Wu, X., Xiong, X., \& Blackwell, W. J. (2013). Overview of intercalibration of satellite instruments. IEEE Transactions on Geoscience and Remote Sensing, 51(3), 1056-1080.

Chander, G., Hewison, T. J., Fox, N., Wu, X., Xiong, X., \& Blackwell, W. J. (2013). Overview of intercalibration of satellite instruments. IEEE Transactions on Geoscience and Remote Sensing, 51(3), 1056-1080.

Chander, G., Mishra, N., Helder, D. L., Aaron, D. B., Angal, A., Choi, T., ... \& Doelling, D. R. (2012). Applications of spectral band adjustment factors (SBAF) for crosscalibration. IEEE Transactions on Geoscience and Remote Sensing, 51(3), 12671281.

Chander, G., Xiong, X. J., Choi, T. J., \& Angal, A. (2010). Monitoring on-orbit calibration stability of the Terra MODIS and Landsat 7 ETM+ sensors using pseudo-invariant test sites. Remote Sensing of Environment, 114(4), 925-939.

Chen, F., Li, J., Wang, C., \& Claverie, M. (2018). A comparison of Sentinel-2A and Sentinel2B with preliminary results. In Proc. IEEE Symp. Geoscience and Remote Sensing. 
Christopherson, J. B., Chandra, S. N. R., \& Quanbeck, J. Q. (2019). 2019 Joint Agency Commercial Imagery Evaluation-Land remote sensing satellite compendium (No. 1455). US Geological Survey.

Chu, M., \& Dodd, J. (2019). Ushering in the New Era of Radiometric Intercomparison of Multispectral Sensors with Precision SNO Analysis. Climate, 7(6), 81.

D'Odorico, P., Gonsamo, A., Damm, A., \& Schaepman, M. E. (2013). Experimental evaluation of Sentinel-2 spectral response functions for NDVI time-series continuity. IEEE Transactions on Geoscience and Remote Sensing, 51(3), 13361348.

Duncan, B. N., Prados, A. I., Lamsal, L. N., Liu, Y., Streets, D. G., Gupta, P., ... \& Burton, S. P. (2014). Satellite data of atmospheric pollution for US air quality applications: Examples of applications, summary of data end-user resources, answers to FAQs, and common mistakes to avoid. Atmospheric environment, 94, 647-662.

ESA, (2018). Sentinel-2 Spectral Response Functions (S2-SRF). https://earth.esa.int/web/sentinel/user-guides/sentinel-2-msi/documentlibrary/-/asset publisher/WkOTKajilSaR/content/sentinel-2a-spectral-responses (Accedido el 02 de Julio de 2020).

ESA, Sentinel 2 Products Specification Document. https://sentinel.esa.int/documents/247904/349490/S2 MSI Product Specifica tion.pdf (Accecido el 18 de Junio de 2020a).

ESA, Sentinel-2

L1C

Data

Quality

Report.

https://sentinel.esa.int/documents/247904/685211/Sentinel-

2 L1C Data Quality Report (Accecido el 22 de Junio de 2020b).

Farhad, M. M., Kaewmanee, M., Leigh, L., \& Helder, D. (2020). Radiometric Cross Calibration and Validation Using 4 Angle BRDF Model between Landsat 8 and Sentinel 2A. Remote Sensing, 12(5), 806.

Gonsamo, A., \& Chen, J. M. (2012). Spectral response function comparability among 21 satellite sensors for vegetation monitoring. IEEE Transactions on Geoscience and Remote Sensing, 51(3), 1319-1335.

Helder, D., Markham, B., Morfitt, R., Storey, J., Barsi, J., Gascon, F., ... \& Lewis, A. (2018). Observations and Recommendations for the Calibration of Landsat $8 \mathrm{OLI}$ and Sentinel 2 MSI for improved data interoperability. Remote Sensing, 10(9), 1340.

Helder, D., Markham, B., Morfitt, R., Storey, J., Barsi, J., Gascon, F., .. \& Lewis, A. (2018). Observations and Recommendations for the Calibration of Landsat $8 \mathrm{OLI}$ and Sentinel 2 MSI for improved data interoperability. Remote Sensing, 10(9), 1340. 
Hird, J. N., DeLancey, E. R., McDermid, G. J., \& Kariyeva, J. (2017). Google Earth Engine, open-access satellite data, and machine learning in support of large-area probabilistic wetland mapping. Remote sensing, 9(12), 1315.

Karlsson, K. G., \& Johansson, E. (2014). Multi-Sensor calibration studies of AVHRRheritage channel radiances using the simultaneous nadir observation approach. Remote Sensing, 6(3), 1845-1862.

Lange, M., Dechant, B., Rebmann, C., Vohland, M., Cuntz, M., \& Doktor, D. (2017). Validating MODIS and sentinel-2 NDVI products at a temperate deciduous forest site using two independent ground-based sensors. Sensors, 17(8), 1855.

Laur, H., \& Liebig, V. (2014). Earth observation satellite missions and data access. In Handbook of Geomathematics.

Liang, J. I., Piper, J., \& Tang, J. Y. (1989). Erosion and dilation of binary images by arbitrary structuring elements using interval coding. Pattern Recognition Letters, 9(3), 201-209.

Liang, S. (2005). Quantitative remote sensing of land surfaces (Vol. 30). John Wiley \& Sons.

Mishra, N., Helder, D., Angal, A., Choi, J., \& Xiong, X. (2014). Absolute calibration of optical satellite sensors using Libya 4 pseudo invariant calibration site. Remote sensing, 6(2), 1327-1346.

Neigh, C. S., McCorkel, J., \& Middleton, E. M. (2015). Quantifying Libya-4 surface reflectance heterogeneity with WorldView-1, 2 and EO-1 Hyperion. IEEE Geoscience and Remote Sensing Letters, 12(11), 2277-2281.

NOAA, (2018). Advisory Committee on Commercial Remote Sensing (ACCRES). https://www.nesdis.noaa.gov/CRSRA/pdf/AACRES meeting 2018 Euroconsult. pdf (Accedido el 24 de Mayo de 2020).

Piper, J. (1985). Efficient implementation of skeletonisation using interval coding. Pattern Recognition Letters, 3(6), 389-397.

Sterckx, S., \& Wolters, E. (2019). Radiometric Top-of-Atmosphere Reflectance Consistency Assessment for Landsat 8/OLI, Sentinel-2/MSI, PROBA-V, and DEIMOS-1 over Libya-4 and RadCalNet Calibration Sites. Remote Sensing, 11(19), 2253.

Tansock, J., Bancroft, D., Butler, J., Cao, C., Datla, R., Hansen, S., ... \& Murdock, T. (2015). Guidelines for radiometric calibration of electro-optical instruments for remote sensing. 
Uprety, S., Cao, C., Xiong, X., Blonski, S., Wu, A., \& Shao, X. (2013). Radiometric intercomparison between Suomi-NPP VIIRS and Aqua MODIS reflective solar bands using simultaneous nadir overpass in the low latitudes. Journal of Atmospheric and Oceanic Technology, 30(12), 2720-2736.

Vescovi, F. D., Lankester, T., Coleman, E., \& Ottavianelli, G. (2015). Harmonisation initiatives of Copernicus data quality control. The International Archives of Photogrammetry, Remote Sensing and Spatial Information Sciences, 40(7), 713.

Wu, M., Yang, C., Song, X., Hoffmann, W. C., Huang, W., Niu, Z., ... \& Yu, B. (2018). Monitoring cotton root rot by synthetic Sentinel-2 NDVI time series using improved spatial and temporal data fusion. Scientific reports, 8(1), 1-12.

Xu, N., Chen, L., Wu, R., Hu, X., Sun, L., \& Zhang, P. (2014, November). In-flight intercalibration of $\mathrm{FY}-3 \mathrm{C}$ visible channels with AQUA MODIS. In Earth Observing Missions and Sensors: Development, Implementation, and Characterization III (Vol. 9264, p. 926408). International Society for Optics and Photonics. 


\section{CAPÍTULO 4. ANÁLISIS DE LA HOMOGENEIDAD ESPACIO - TEMPORAL DEL PICS LIBYA-4, PARA LA LOCALIZACIÓN DE UN ÁREA DE CALIBRACIÓN OPTIMIZADO}

\subsection{Introducción}

Los datos y productos de teledetección mejoran la toma de importantes decisiones en diferentes ámbitos como la política, defensa, industria, agricultura o clima (BeckerReshef et al., 2010; Jha et al., 2008; Xiong et al., 2012; Liu et al., 2014; Corbane et al., 2010; NOAA, 2018). Hay que tener en cuenta que los sensores ópticos de los satélites, proporcionan información diversa, debido a las características únicas de diseño de los instrumentos científicos que llevan a bordo, así como por los objetivos establecidos para cada misión. Los instrumentos ópticos espaciales, se caracterizan y calibran antes del lanzamiento durante la fase de Pre-Lanzamiento. Incluso con el mismo diseño y proceso de fabricación, no hay dos sensores idénticos (Chander et al., 2013).

Esta diversidad sin precedentes de datos ópticos (NOAA, 2018; Denis et al., 2017), hace más evidente la necesidad de mejora en la interoperabilidad y armonización de los datos de satélite para que puedan ser comparables, a pesar de provenir de fuentes de datos dispares (Vescovi et al., 2015; Mhangara \& Mapurisa, 2019). Un factor clave para la consecución de estos objetivos es la calibración de los sensores. Una vez en órbita, y debido a la degradación en el entorno espacial, es necesario el uso de datos adquiridos en blancos terrestres de referencia como parte de su procedimiento de calibración (Lyapustin et al., 2014). La calibración usando blancos terrestres, es una actividad esencial tanto para satélites con sistemas de calibración on board, como para los que no la tienen (Mishra et al., 2016). Los blancos terrestres utilizados para la calibración, también son utilizados para realizar la comparación entre sensores.

Las metodologías basadas en PICS para la calibración absoluta posterior al lanzamiento, la cross-calibration con otros sensores y el monitoreo de la estabilidad radiométrica de sensores ópticos de satélite, ha sido ampliamente utilizadas por el sector de la teledetección en las últimas décadas (Cosnefroy et al., 1996; Chander et al., 2010; Rao \& Chen, 1999; Heidinger et al., 2002; Chander et al., 2013).

Utilizando los datos del Meteosat-4, Cosnefroy et al. (Cosnefroy et al., 1996) buscaron superficies espacialmente uniformes y temporalmente estables, encontrando 20 lugares desérticos en el norte de África y Arabia Saudí. En el año 2008, CEOS seleccionó seis de esas ubicaciones como zonas de calibración preferente (Kharbouche \& Muller, 2016). 
Estos seis PICS, se encuentran localizados en el Sahara: Argelia-3, Argelia-5, Libya-1, Libya-4, Mauritania-1 y Mauritania-2 (Figura 58).
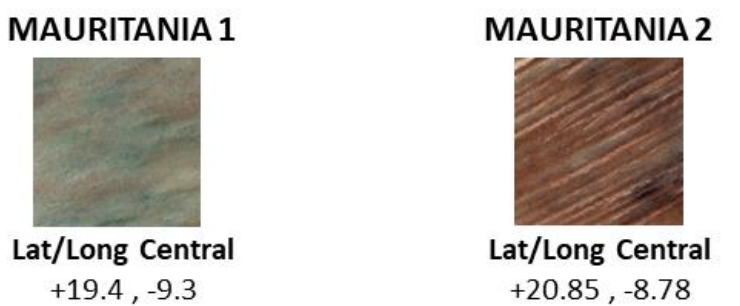

\section{LIBYA 1}

$+19.4,-9.3$

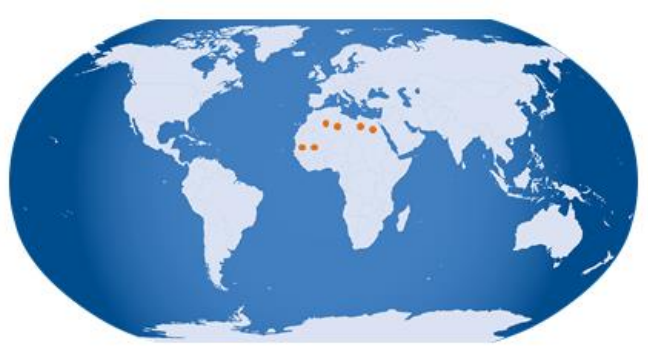

Lat/Long Central

$+24.42,+13.35$

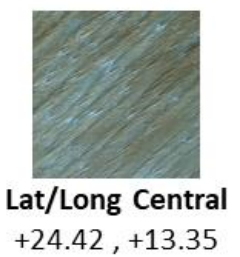

ALGERIA 3

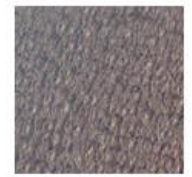

Lat/Long Central $+30.32,+7.66$

\section{ALGERIA 5}

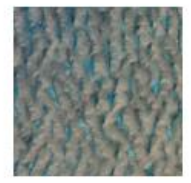

Lat/Long Central $+31.02,+2.23$
LIBYA 4

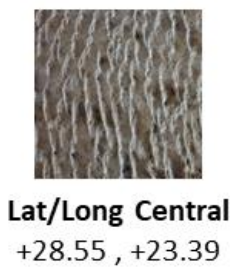

Figura 58. PICS recomendados por CEOS.

Existen otros sitios de calibración (varios de ellos situados en desiertos) distribuidos por todo el mundo (Helder et al., 2010; Berthelot, 2015) y algunos de ellos instrumentados, pero de forma general con una extensión espacial mucho menor. En la Figura 59 se muestran los sitios de referencia aprobados por CEOS para la calibración PostLanzamiento de sensores ópticos de satélites. 


\section{Distribution of World-Wide Radiometric Sites}

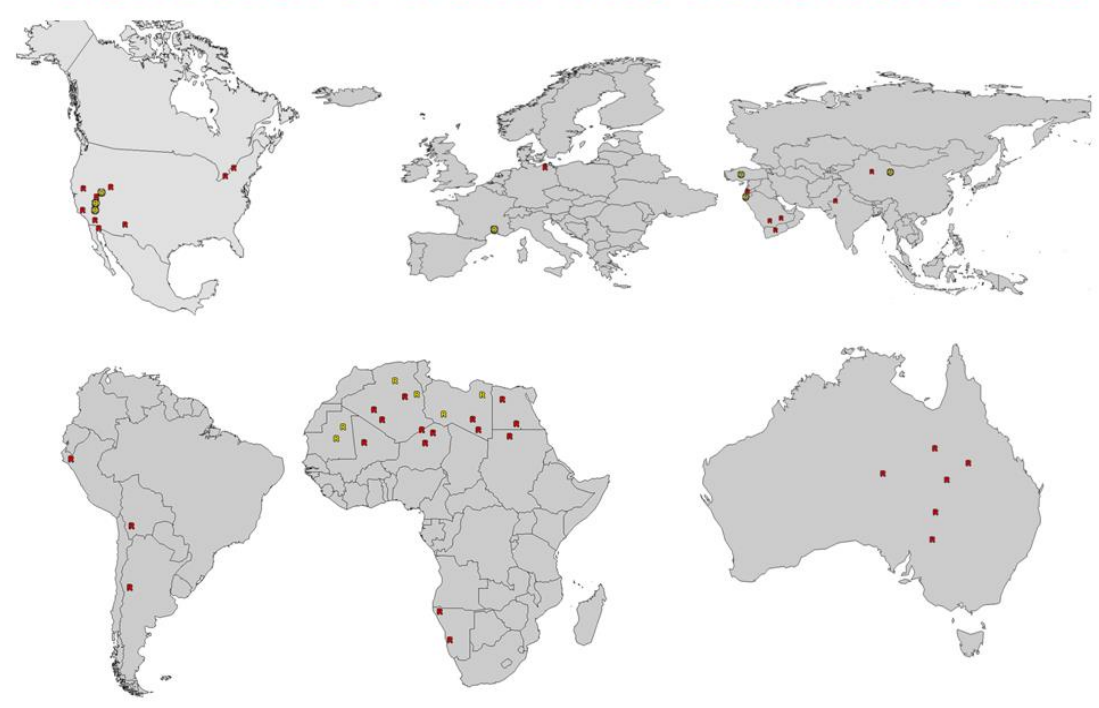

Figura 59. Distribución de targets de calibración. Fuente: Adaptada de (USGS, 2020a)

Dentro de los seis PICS recomendados por CEOS, Libya-4 es el más utilizado para las actividades de Cal/Val debido a una mayor uniformidad y estabilidad que el resto de los PICS (Mishra et al., 2014). Debido a ello, se ha elegido Libya-4 entre los diferentes PICS posibles. El subárea del PICS libya-4, descrita en esta tesis como Libya-4 AOI, tiene una superficie de $100 \times 100 \mathrm{~km}^{2}$, siendo especialmente útil para sensores de baja y media resolución. A medida que se ha dispuesto de instrumentos de mayor resolución, varios autores comenzaron a utilizar diferentes subáreas, ya sea, porque consideraban que el AOI de Libya-4 era demasiado grande o simplemente porque el swath del sensor era demasiado estrecho para adquirir la superficie completa (Sterckx et al., 2013; Govaerts et al., 2004; Bhatt et al., 2004). CEOS detectó la necesidad de fijar un área más pequeña y definió una de $20 \times 20 \mathrm{~km}^{2}$ (CEOS AOI) como un subconjunto del área dentro de Libya4 AOI (Lacherade et al., 2013).

Govaerts (2015) demostró que los tamaños de los AOI en el rango de $10 \times 10 \mathrm{~km}^{2}$ en Libya-4, tenían menos estabilidad en lo que respecta a las propiedades ópticas de la superficie que los AOI de mayor superficie debido al efecto duna. Esta problemática podría influir en la calibración de los sensores después del lanzamiento. El uso de un AOI de tamaño óptimo reduce la posible incertidumbre generada por el efecto duna, mejorando la estabilidad de la calibración indirecta (Govaerts, 2015). A parte de la característica intrínseca de los PICS de variabilidad estacional a corto plazo, la denominada season trend de ciclo anual, Tuli et al. (2019) mostraron que hay una variabilidad temporal a largo plazo en algunos PICS, siendo necesaria su monitorización.

Actualmente, una de las recomendaciones realizadas por PICSCAR es la falta de acuerdo en la utilización de un mismo AOI de calibración dentro de cada PICS como se muestra en la Figura 60 (ESA, 2017; Berthelot \& Henry, 2017). PICSCAR considera una acción 
prioritaria la armonización y actualización de los vigentes targets de referencia de calibración (ESA, 2017; Berthelot \& Henry, 2017).

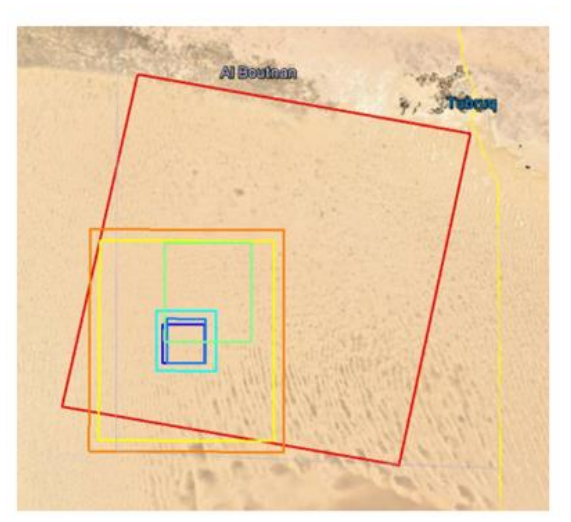

11 users

7 different site locations

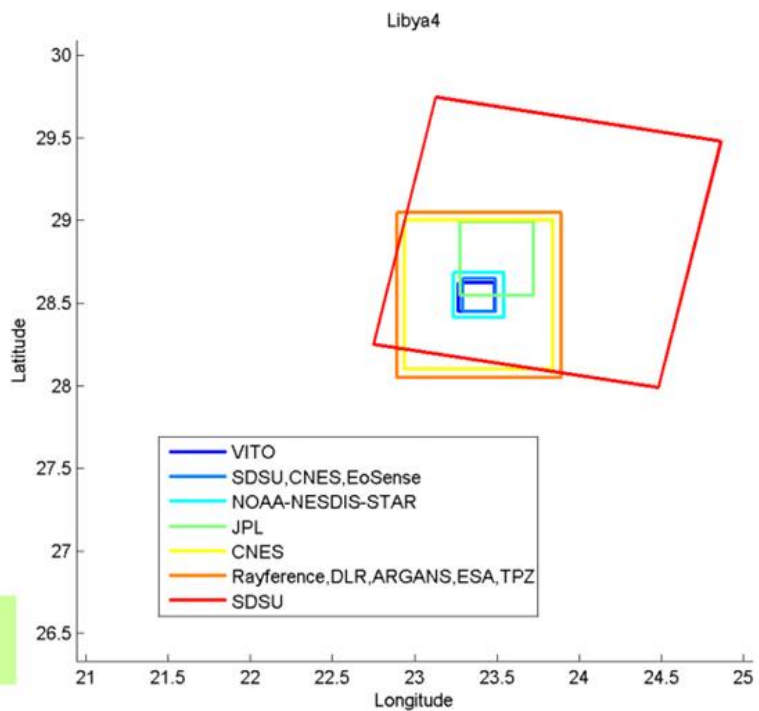

Figura 60. Diferentes AOls utilizados en Libya-4. Fuente: Berthelot \& Henry (2017) basadas en PICS para sensores de resolución media y alta, gracias a la ubicación de un OGT dentro de Libya-4 con la mayor estabilidad espacio-temporal posible. Para ello, se ha estudiado la variabilidad de Libya-4 de una amplia serie temporal de datos combinados de L8-OLI y S2-MSI, utilizando una nueva metodología numérica. Se ha utilizado el CV y un algoritmo de optimización quasi-Newton (Broyden, 1970; Fletcher, 1970; Shanno, 1970) combinado con una técnica de optimización global Basin-Hopping (Wales \& Doye, 1997), para limitar un área de búsqueda lo suficientemente pequeña. Posteriormente se realizó un refinamiento final por brute-force (Verma et al., 2014; Sen \& Kumar, 2019), con el propósito de definir la zona con el tamaño y la estabilidad espacio-temporal óptima.

\subsection{Materiales y Métodos}

\subsection{1. Área de Estudio: Libya-4}

Libya-4, fue uno de los seis PICS recomendados por el Grupo de Trabajo CEOS sobre Calibración y Validación (WGCV) (Teillet \& Chander, 2010) junto con Mauritania-1, Mauritania-2, Argelia-3, Argelia-5 y Libya-1. Se encuentra localizado en el denominado Gran Mar de Arena (28.55 $\left.{ }^{\circ} \mathrm{N}, 23.39^{\circ} \mathrm{E}\right)$ a una altura promedio de $118 \mathrm{~m}$ sobre el nivel del mar. El área de estudio está cubierta por dunas de arena sin vegetación, presenta una alta estabilidad atmosférica con un bajo contenido de aerosoles, así como una baja presencia de turbidez (Choi et al., 2014). Libya-4 reúne todas las características de un buen PICS (Chander et al., 2007): anisotropía de baja reflectancia, alta reflectancia superficial, elevada uniformidad espacial y temporal, y una gran superficie de suelo. 
Para L8-OLI, el sitio CEOS de Libya-4 está ubicado en el path 181 y row 40 (Figura 61) referenciados en el WRS-2 (USGS, 2020b) con un área de escena aproximada de $185 \times$ $180 \mathrm{~km}^{2}$ (USGS, 2020c). Para el caso de S2-MSI, para cubrir completamente el área de Libya-4, es necesario usar 6 tiles (34RFT, 34RFS, 34RGT, 34RGS, 35RKN y 35RKM), referenciados al MGRS, con un tamaño aproximado por tile de $100 \times 100 \mathrm{~km}^{2}$ y $10 \mathrm{~km}$ de superposición (Figura 62) (Gascon et al., 2017).

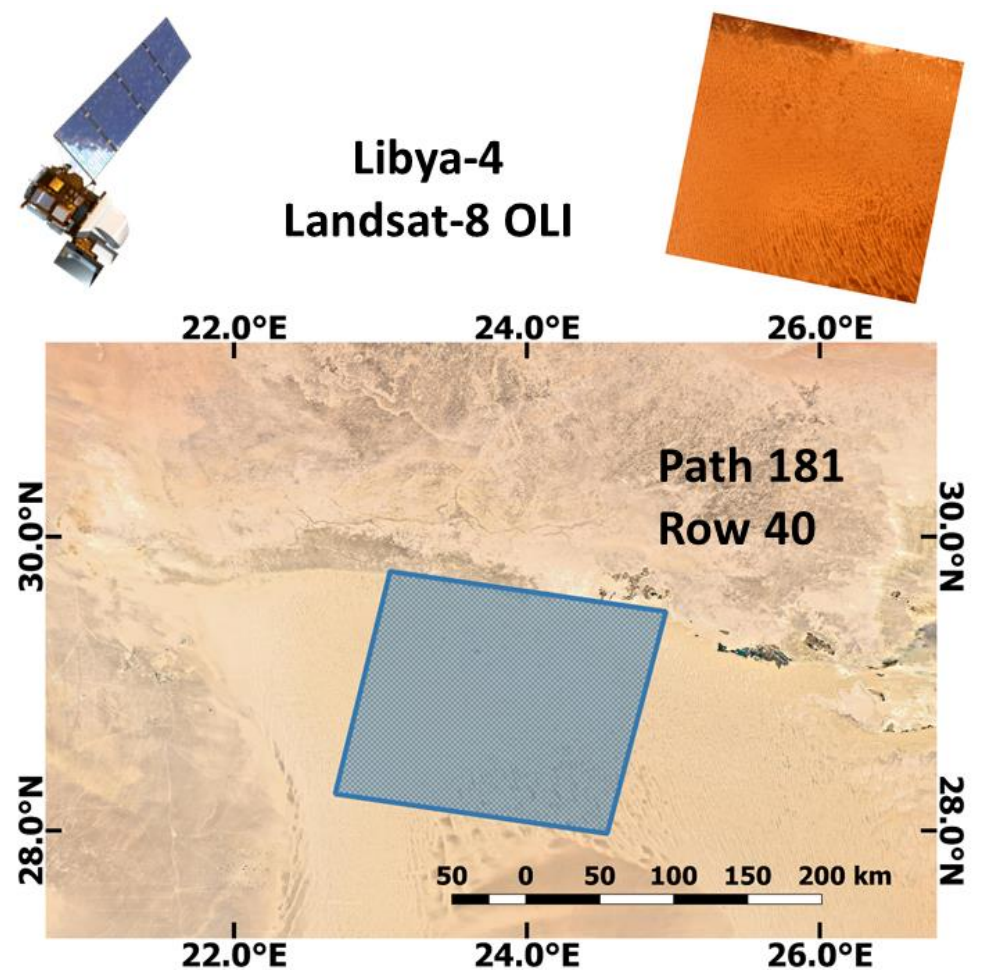

Figura 61. Vista de Google Earth del AOI de L8 en Libya-4

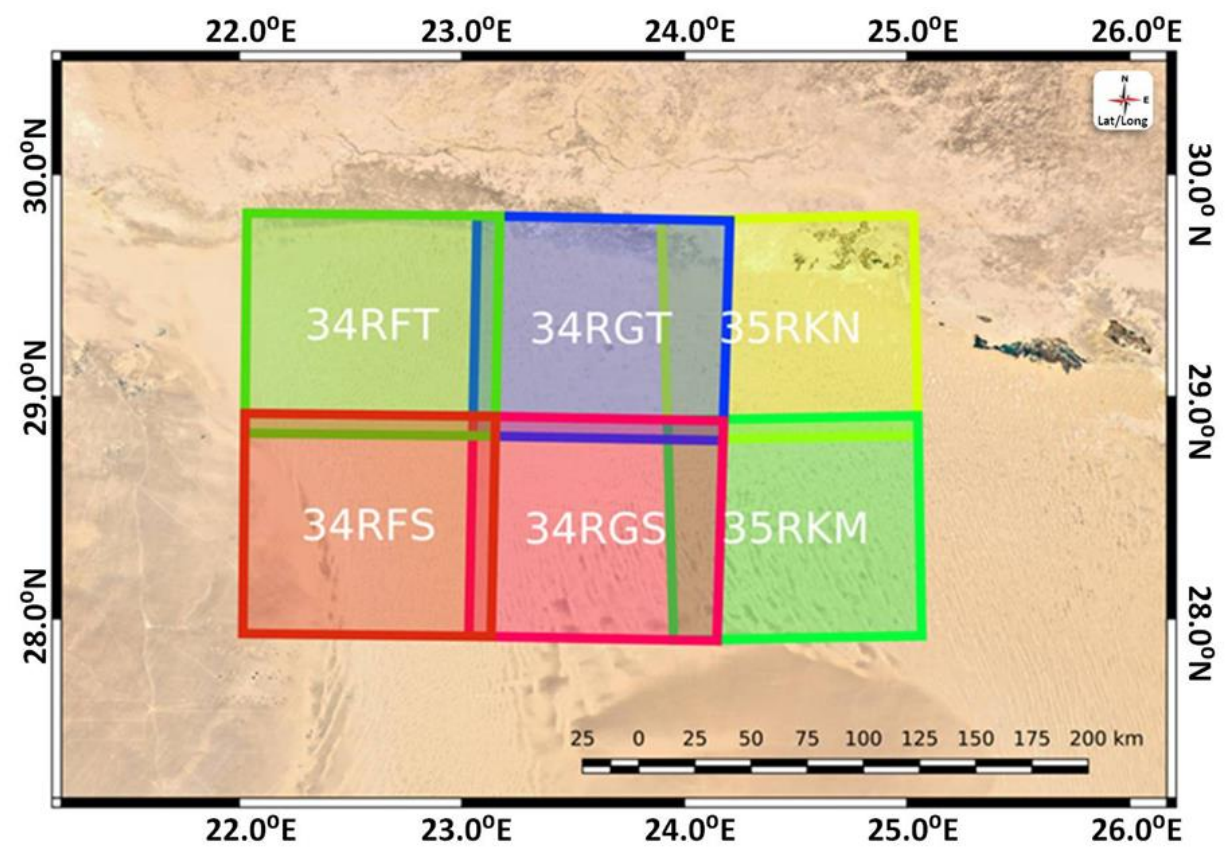

Figura 62. Vista de Google Earth de los tiles de S2 en el área de estudio de Libya-4 
CEOS definió un AOI de $20 \times 20 \mathrm{~km}^{2}$ que permitía a sensores de mayor resolución que no podían cubrir el área completa de Libya-4 tener una zona de referencia más localizada (Lacherade et al., 2013). El AOI de L8 y el tile 34RGS de S2 contienen el CEOS AOI.

Además de los diferentes $\mathrm{AOI}$ mencionados anteriormente, se tuvo en cuenta la presencia de los datos hiperespectrales del sensor Hyperion del satélite EO-1 actualmente no operativo (Figura 63). Los datos de Hyperion han permitido durante años, una perfecta caracterización espectral de la superficie de Libya-4 debido a las 220 bandas espectrales continuas que cubren el VNIR y el SWIR, que van desde $400 \mathrm{~nm}$ a $2500 \mathrm{~nm}$ con un ancho de banda de aproximadamente $10 \mathrm{~nm}$ (USGS, 2020d).

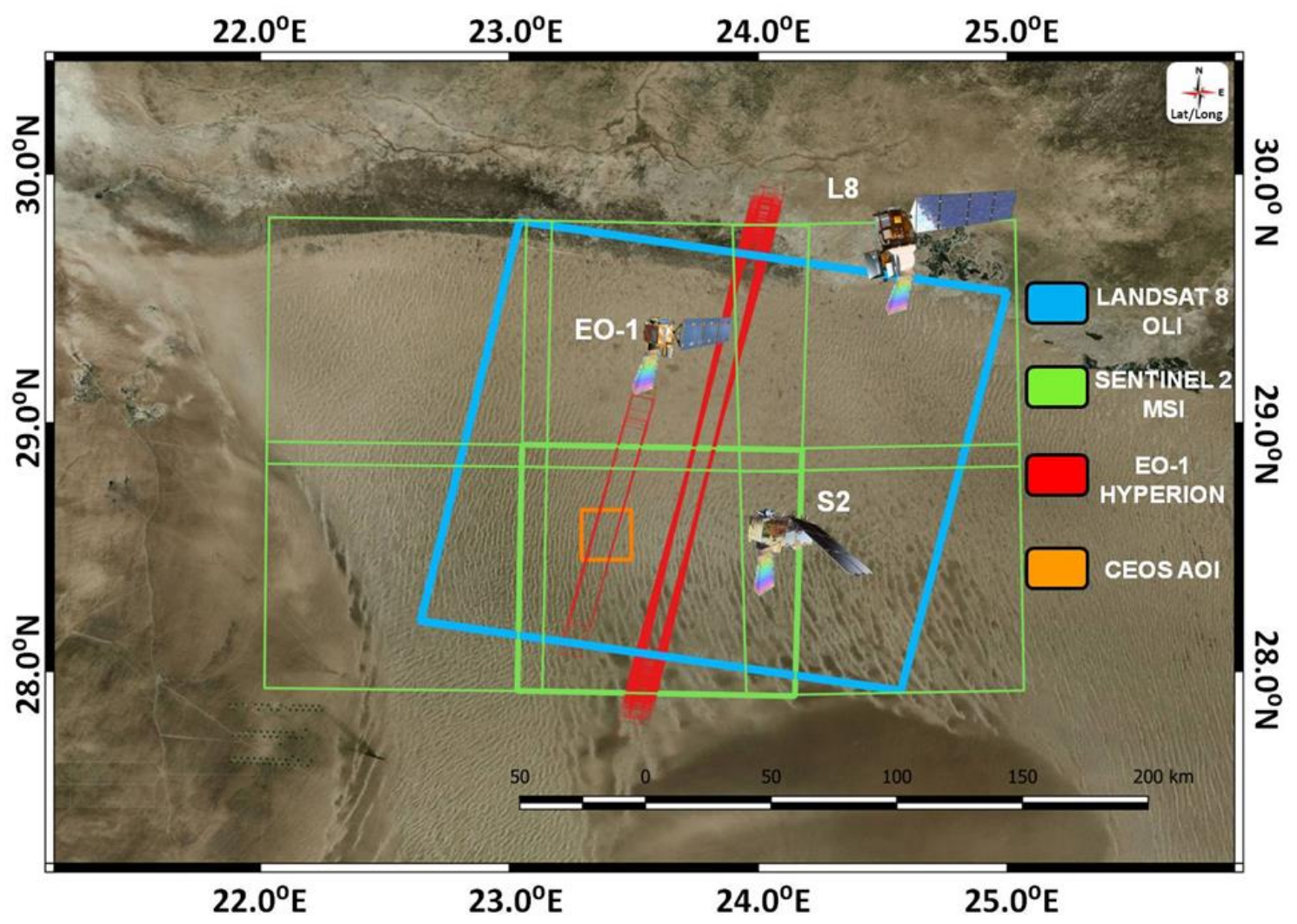

Figura 63. Representación en Google Earth de la ubicación de las diferentes áreas de interés L8 y S2 (AOI), así como las zonas de adquisición del sensor Hyperion en Libya-4.

El objetivo de este análisis era encontrar un OGT común para L8 y S2 en Libya-4, por esa razón, se utilizaron los datos del tile WRS-2 181/40 de L8, y para S2, los datos de los 6 mosaicos MGRS (34RFT, 34RFS, 34RGT, 34RGS, 35RKN y 35RKM) que cubren específicamente el área L8 en Libya-4 más un buffer añadido. Es importante señalar que las imágenes L8 son ligeramente más grandes que el tile WRS-2 (Figura 63). 


\subsubsection{Satélites y Sensores utilizados}

Para evaluar la homogeneidad espacial y estabilidad temporal de Libya-4, se seleccionaron los datos L8-OLI y S2-MSI por su excelente calidad (Gascon et al., 2017; Roy et al., 2014).

Para el caso de L8, se utilizaron productos de reflectancia TOA calibrados, pertenecientes a la L8 Collection-1 Tier 1 referenciados al sistema WRS-2 en la proyección UTM/WGS84. Se seleccionarón también las principales bandas en el rango VNIR de L8, es decir, red, green, blue y $N I R$, debido a que son las más utilizadas por la mayoría de sensores de EO. Este producto se encuentra calibrado y orto rectificado radiométricamente y geométricamente utilizando puntos de control en tierra y también a partir de un modelo digital del terreno. Una caractererística fundamental del mismo, es su excelente precisión y calidad, utilizándose especialmente para comparar grandes series de datos a nivel de píxel (USGS, 2020d). En el año 2017, el USGS realizó un reprocesamiento de los datos para toda la serie Landsat, creando la denominada Collection-1, que es el standard de datos recomendado actualmente por el USGS y la colección de datos utilizada en esta tesis (Dwyer et al., 2018; USGS, 2020d).

Como en el caso de L8, para S2 se seleccionaron para este estudio las bandas blue, green, red y NIR, las cuales poseen una resolución espacial de $10 \mathrm{~m}$. Entre las dos bandas NIR de S2 (8 - 8A), se utilizó la banda NIR ancha de MSI (8) para mantener la consistencia espacial de $10 \mathrm{~m}$ de resolución con el resto de las bandas S2. Para los datos S2, se utilizó el producto L1C que está estructurado en tiles, los cuales, están referenciados al sistema MGRS y con un tamaño de imágenes de $100 \times 100 \mathrm{~km}^{2}$. Estos productos se entregan en la proyección UTM/WGS84. Las imágenes están en valores de reflectancia TOA multiplicados por 10.000 (ESA, 2020).

Las características principales de las bandas utilizadas de L8-OLI y S2-MSI se resumen en la Figura 64 y Tabla 16.

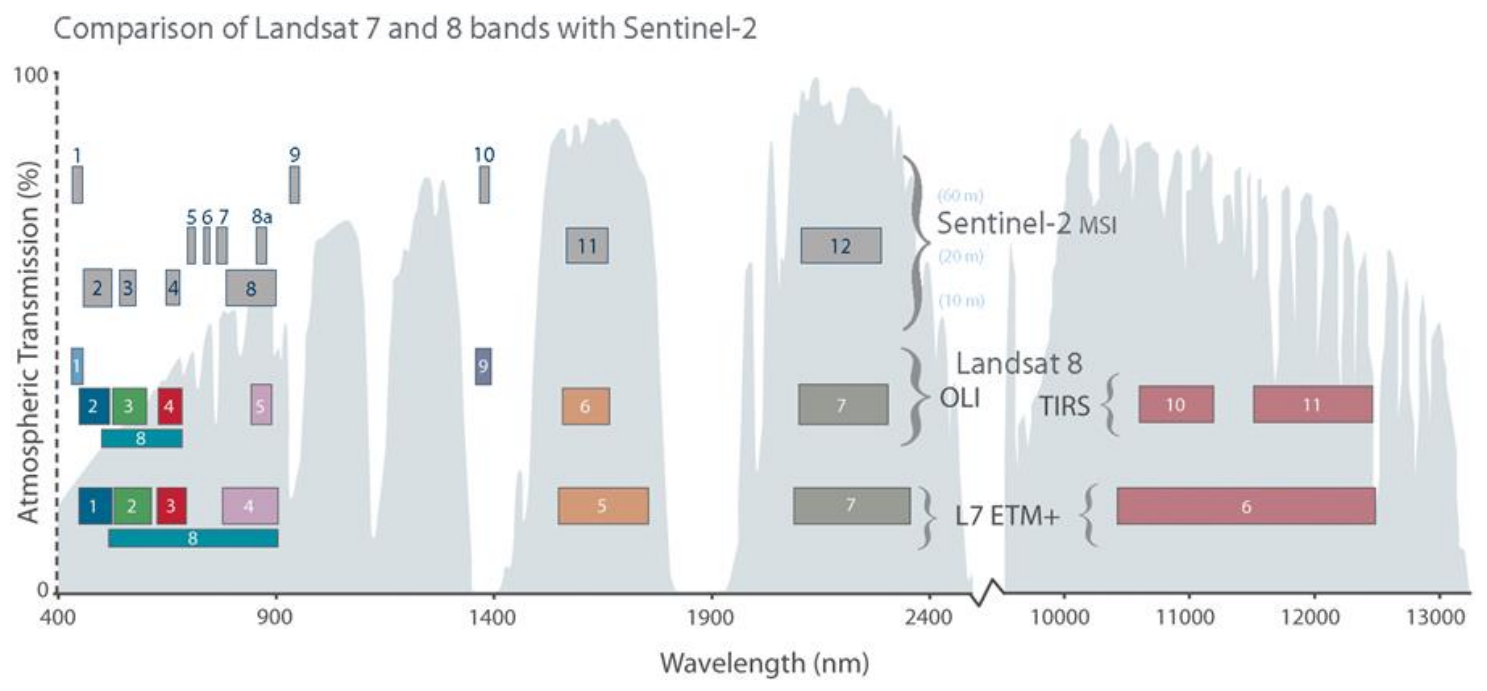

Figura 64. Comparativa de las Bandas de los Satélites Landsat-7, Landsat-8 y Sentinel 2. 
Tabla 16. Bandas espectrales utilizadas y sus características de resolución espacial para los sensores Landsat-8 OLI y Sentinel-2 A/B.

\begin{tabular}{ccccc}
\hline & Banda \# & Banda & $\begin{array}{c}\text { Resolución } \\
\text { Espacial } \\
(\mathbf{m})\end{array}$ & $\begin{array}{c}\text { Longitud de Onda } \\
\text { Central } \\
(\mathbf{n m})\end{array}$ \\
\hline \multirow{3}{*}{ Landsat 8 OLI } & 2 & Blue & 30 & 482.0 \\
& 3 & Green & 30 & 561.4 \\
& 4 & Red & 30 & 654.6 \\
\hline & 5 & NIR & 30 & 864.7 \\
\hline Sentinel 2 A MSI & 2 & Blue & 10 & 492.4 \\
& 3 & Green & 10 & 559.8 \\
& 4 & Red & 10 & 664.6 \\
\hline & 8 & NIR & 10 & 832.8 \\
\hline & & & & \\
Sentinel 2 B MSI & 2 & Blue & 10 & 492.1 \\
& 3 & Green & 10 & 559.0 \\
& 4 & Red & 10 & 664.9 \\
& 8 & NIR & 10 & 832.9 \\
\hline
\end{tabular}

\subsubsection{Coeficiente de Variación (CV)}

EI CV es un operador que se ha utilizado por diferentes autores y aplicaciones, como medida de la homogeneidad espacial (Kneubühler et al., 2006; De Vries et al., 2007; Gu et al., 1990), así como, para la medición de la homogeneidad temporal (Helder et al., 2010). El CV puede definirse como el cociente entre la desviación típica y la media de los valores; por lo tanto, no tiene unidades al ser adimensional. EI CV suele representarse en forma de porcentaje. En la Ecuación 8 se muestra la fórmula del CV.

$$
C V=\frac{\sigma}{|\bar{x}|} \text { (Eq. 8) }
$$

Donde $|\bar{x}|$ es el valor medio y $\sigma$ es la desviación estándar de la muestra.

En este trabajo, se ha aplicado el CV como indicador de la homogeneidad radiométrica de Libya-4, tanto temporal como espacialmente.

\subsubsection{Google Earth Engine}

Google Earth Engine (GEE) contiene un catálogo de datos a escala de petabytes de múltiples satélites. Esta plataforma pone toda esta información, sus capacidades de análisis y de computación en la nube a disposición de los científicos, investigadores y desarrolladores del todo el mundo con el propósito de aplicarlos en diferentes campos, cuyo eje central es el estudio de la superficie de la Tierra (GEE, 2020; Gorelick et al., 2017).

Esta disponibilidad de datos y de procesamiento en la nube, elimina uno de los mayores problemas en las investigaciones, que es la necesidad de contar con una compleja, 
actualizada y costosa infraestructura de almacenamiento y procesamiento de datos, que permita llevar a cabo toda la algoritmia de la investigación.

Desde el año 2008 GEE ha puesto a disposición de los usuarios de forma gratuita todo el catálogo de datos de la serie Landsat. GEE ha continuado ampliando el conjunto de datos añadiendo nuevos sensores como es el caso de los datos S2 (GEE, 2020) (Figura 65).

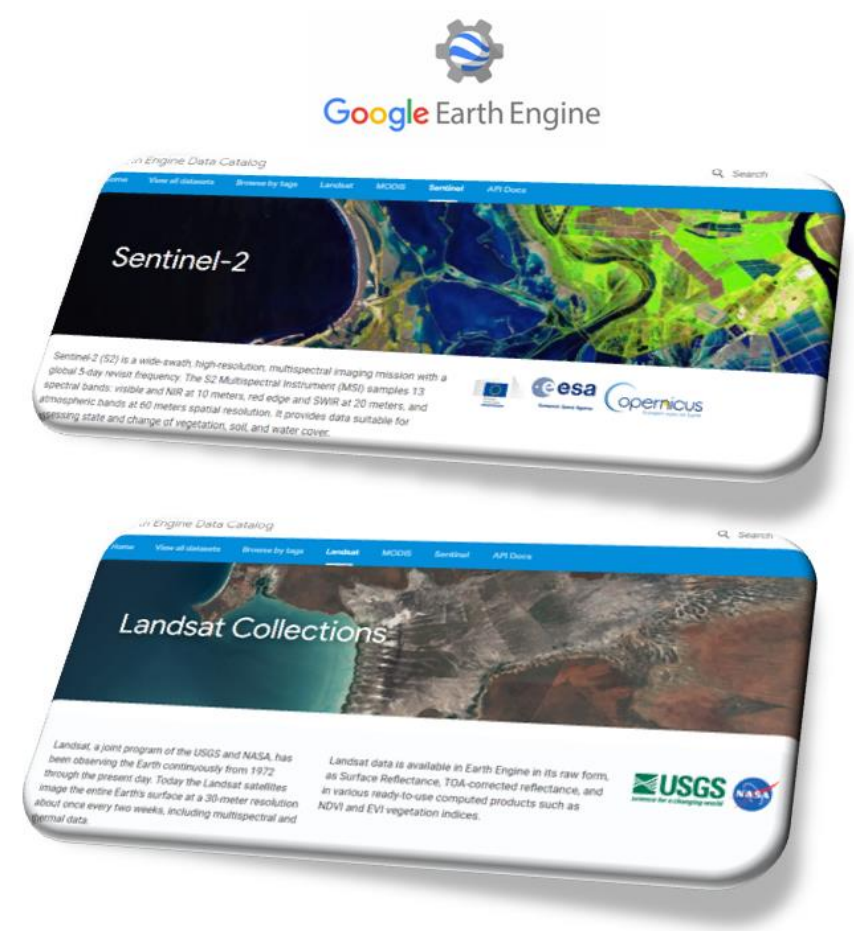

Figura 65. Ejemplo de datos disponibles en GEE.Fuente: Adaptado de GEE (2020)

En esta tesis doctoral se ha utilizado GEE para generar las imágenes CV de los datos de L8 y S2, dada su excelente capacidad de computación y su flexibilidad en la nube.

\subsubsection{Uniformidad Espacial y Temporal}

Con el propósito de caracterizar y localizar el OGT sobre Libya-4 que cumpliese con los criterios de mayor homogeneidad posible tanto a nivel espacial como temporal, fue necesario utilizar una amplia serie temporal de datos L8 y S2. Los datos de los sensores L8-OLI y S2-MSI se utilizaron desde el comienzo de las misiones hasta los últimos datos disponibles en 2018.

Uno de los aspectos importantes a tener en cuenta en las imágenes de L8-OLI y S2-MSI es la nubosidad. Este fenómeno tiene un gran impacto en el CV al contaminarse la medida y por ello los cálculos de homogeneidad. Se ha demostrado que el uso de un operador humano para discernir de manera fiable los diferentes tipos de nubes, sombras y otros elementos relacionados presentes en las imágenes, es fundamental para validar la calidad de las imágenes (Coluzzi et al., 2018). Por esta razón, los conjuntos de datos que muestraban nubes, sombras de nubes, polvo en suspensión y otras características obvias que pudieran afectar negativamente al análisis, se filtraron por 
medio de la técnica de fotointerpretación a partir del quicklook de las imágenes. Para que una imagen fuera considerada válida era necesario que el AOI cubierto por L8 sobre Libya-4 estuviera despejado de nubes o de cualquier otro tipo de fenómeno que pudiese afectar al análisis. Otra razón importante para realizar una evaluación visual mediante un fotointérprete que discrimine las imágenes afectadas por nubes, sombras, polvo en suspensión u otros elementos problemáticos, era la posibilidad de los falsos positivos que presentan los algoritmos de detección de nubes, especialmente para superficies de alta reflectancia como los desiertos (Sun et al., 2017). También fue importante tener en cuenta, que a diferencia de L8 que posee el sensor TIRS, los satélites S2 no tienen ninguna banda térmica en el sensor MSI, por lo que la máscara de nubes generada por S2 resultaba menos fiable (Helder et al., 2018).

Los quicklooks de L8 fueron descargadas del Earth Resources Science and Observation Data Center (EROS) del USGS (https://earthexplorer.usgs.gov/) y los quicklooks de S2 fueron descargados del Copernicus Hub (https://scihub.copernicus.eu/). Un total de 598 escenas de S2 y 64 escenas de L8 fueron seleccionadas para posteriormente ser utilizadas en GEE. En la Figura 66 se presenta el flujo de trabajo del proceso de selección del data set de imágenes.

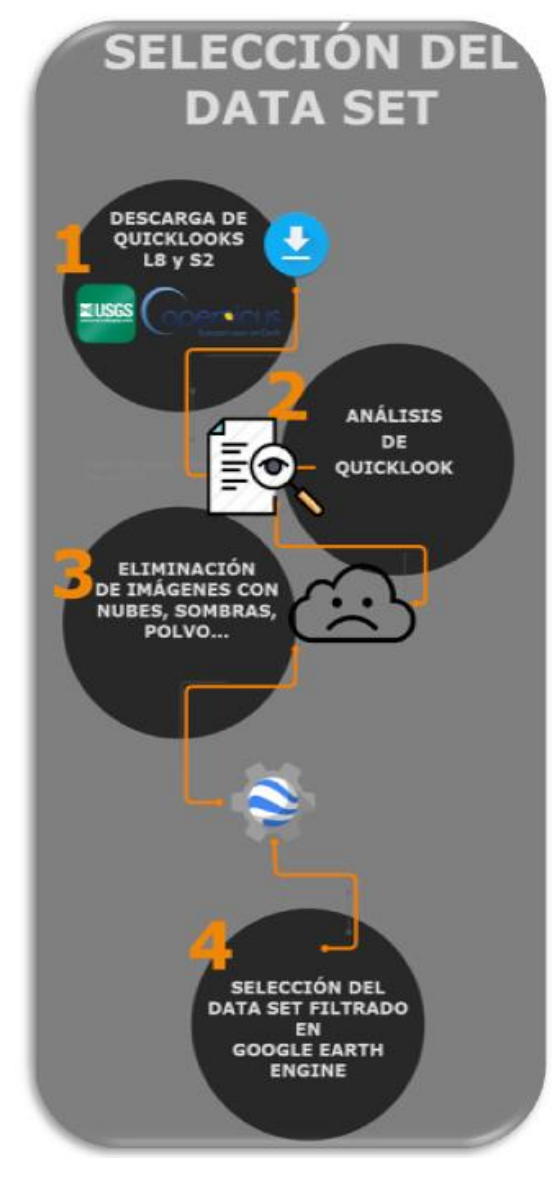

Figura 66. Flujo de trabajo del proceso de selección del data set de imágenes a utilizar en GEE. 
Una vez fue seleccionado el data set de imágenes de L8 y S2, hubo que definir un coeficiente que permitiese el estudio de la homogeneidad espacial y temporal. Con la intención de minimizar operaciones y capacidad de procesado, era recomendable que dicho coeficiente permitiese conocer de forma simultánea ambos parámetros de homogeneidad.

Se tomó la imagen de reflectancia TOA de la banda $i$, de la adquisición $j$, y se convolucionó usando el operador de desviación estándar $\sigma$ y un kernel cuadrado de 300 $\mathrm{m}$ para obtener la imagen $\sigma^{i j}$. Se repitió el mismo proceso utilizando el operador promedio $\bar{x}$ para obtener la imagen $\bar{x}^{i j}$. Por lo tanto, después de realizar estas operaciones espaciales, se generaron dos rasters por cada banda de la imagen y adquisición. Por conveniencia computacional, se organizaron en un data cube, donde el eje $j$ (adquisición) representa la componente de tiempo. Los datos se aplanaron en el data cube a través de la dimensión de tiempo y los pares de imágenes se combinaron, siguiendo la Ecuación 9, que define el CV espacio-temporal (CV $\mathrm{ST}_{\mathrm{ST}}$. El resultado fue una imagen $\mathrm{CV}_{\text {ST }}$ por banda.

$$
C V_{\mathrm{ST}_{n m}^{i}}^{i}=\frac{\sqrt{\sum_{j=1}^{J}\left(\sigma_{n m}^{i j}\right)^{2}}}{\sum_{j=1}^{J} \bar{x}_{n m}^{i j}} \text { (Eq. 9) }
$$

Donde $(\mathrm{n}, \mathrm{m})$ son las coordenadas de píxel de la banda $i$, y $J$ es el número de adquisiciones. La presencia de las coordenadas de píxel $(n, m)$ en la ecuación del $C_{\text {ST }}$, indica de una manera clara, que las operaciones están basadas a nivel de píxeles.

Es muy importante resaltar que estas operaciones no están vinculadas específicamente a los sensores L8-OLI y S2-MSI, pudiéndose aplicar a cualquier otro tipo de sensor. Cada sensor fue considerado de forma independiente, realizándose el cálculo para las bandas específicas de cada uno de ellos. También es importante tener en cuenta, que las convoluciones que se realizaron previamente redujeron el impacto de pequeños errores geométricos que se pueden producir en las operaciones basadas a nivel de píxel.

El CV $\mathrm{ST}_{\text {TT }}$ permite la comparación de la homogeneidad de diferentes bandas espectrales. La Figura 67 muestra el flujo de trabajo del análisis de la uniformidad espacial y temporal de Libya-4, aplicado para el caso concreto de la banda NIR de L8. 


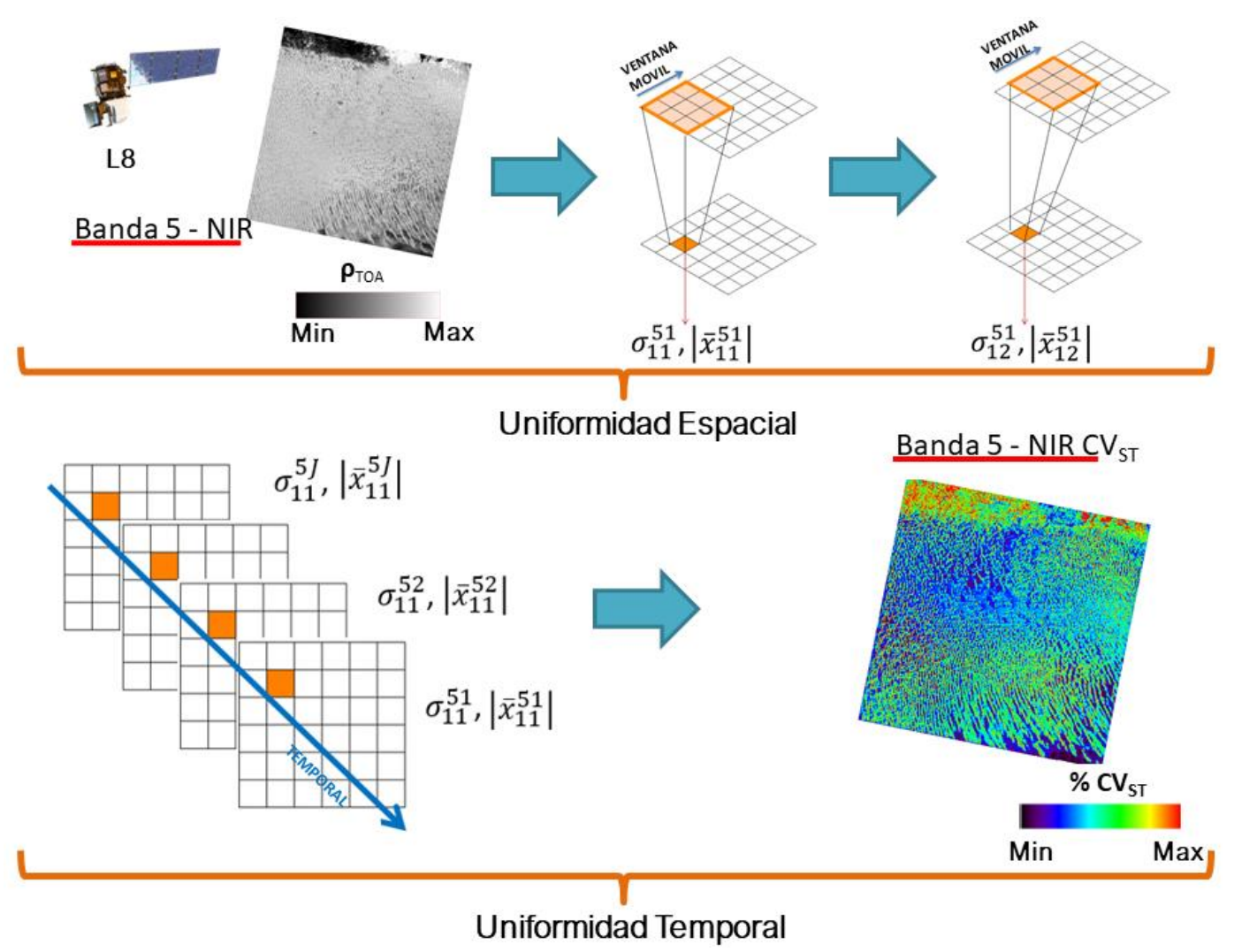

Figura 67. Ejemplo del flujo de trabajo para cálculo del CV $V_{S T}$ de la banda NIR de L8.

Una vez que se generaron las imágenes $\mathrm{CV}_{\mathrm{ST}}$ de las bandas red, green, blue y NIR (RGBNIR) de L8 y S2, se procedió a discretizar el área de estudio, enfocando el análisis en zonas con superficie cuadrada con un $\mathrm{CV}_{S T}$ promedio más bajo para cada banda. Para ello se combinó un algoritmo de optimización quasi-Newton (Battiti \& Masulli, 1990), conocido como el método de Limited Memory Broyden Fletcher Goldfarb Shanno (LM BFGS), combinado con el Basin-Hopping Global Optimization Technique (BH-GOT) (Iwamatsu \& Okabe, 2004).

El algoritmo LM-BFGS es un algoritmo de gradiente básico, mientras que el método de optimización de BH-GOT se basa en transformar la superficie de energía potencial en el mínimo local más cercano a cada punto mediante un algoritmo de minimización local, en este caso particular, en un AOI con el mínimo $\mathrm{CV}_{\text {ST }}$ promedio. La superficie se analiza desplazando las coordenadas aleatoriamente y cada desplazamiento es decidido de acuerdo con su probabilidad de Boltzmann (Díaz, 2017). Una ventaja importante de BHGOT con respecto a otros algoritmos, es que BH-GOT no se queda atrapado indefinidamente en un pozo correspondiente a un mínimo local (Figura 68). 


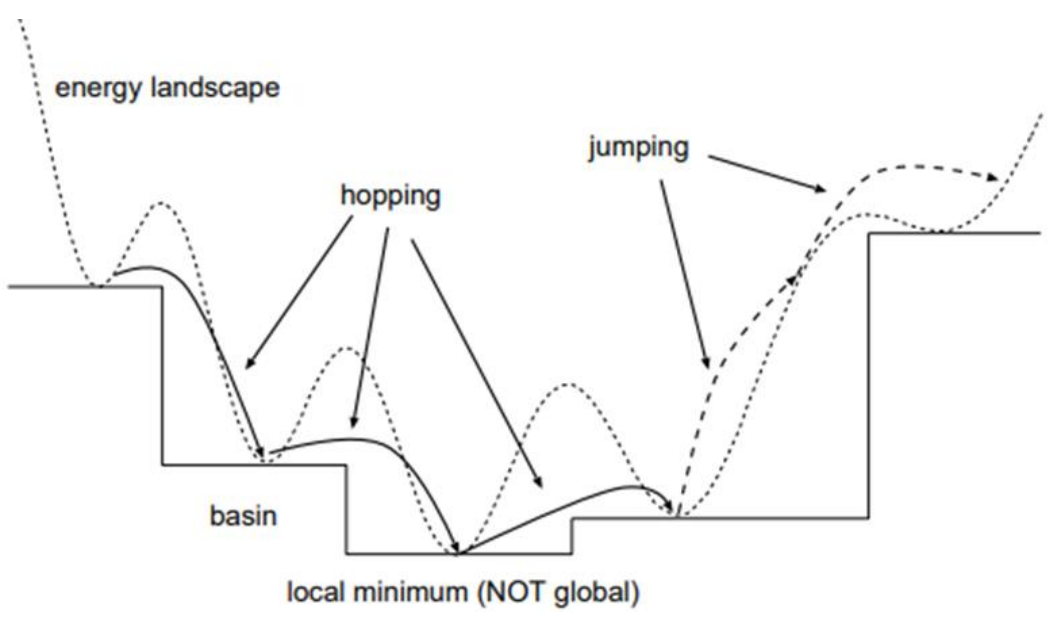

Figura 68. Ejemplo de funcionamiento del BH-GOT.

La técnica de optimización BH-GOT permitió encontrar el mínimo de una función, en este caso, el CV $\mathrm{CV}_{\text {ST }}$ promedio mínimo de una zona cuadrada para cada banda RGBNIR de L8 y S2, donde la ubicación y el área son variables. La ubicación estaba limitada a la extensión del WRS-2 path 181 y row 40 tile que incluía el AOI de Libya-4, y el área a un mínimo de $20 \times 20$ km2 (Figura 69), lo suficientemente grande como para evitar el efecto duna identificado por Govaerts (Govaerts, 2015), y un máximo de $100 \times 100 \mathrm{~km}^{2}$. Esta técnica de optimización se ha aplicado en diferentes áreas, como física, química, matemáticas y biología (Walles \& Doye, 1997; Walles, 2003; Li \& Scheraga, 1987; Walles \& Scheraga, 1999).

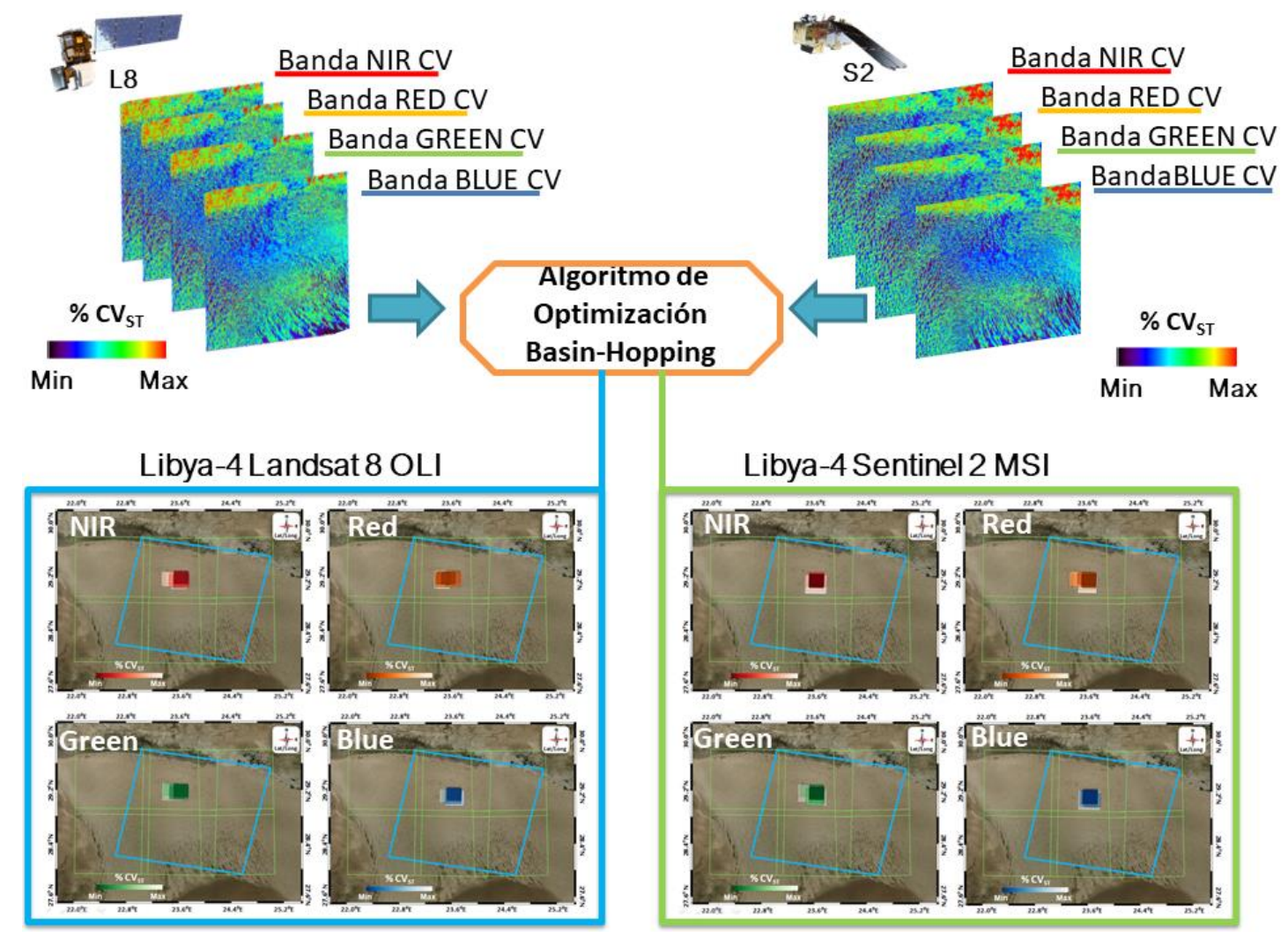

Figura 69. NIR, red, green y blue Libya-4 AOIs de S2 y L8, encontrados por optimización. 
Para cada banda RGBNIR de L8 y S2 de forma individual, se obtuvieron las diferentes geometrías optimizadas. Cada AOI optimizado tenía un tamaño diferente, con una extensión mínima de $20 \times 20 \mathrm{~km}^{2}$. Una vez generados estas AOI optimizados para cada banda de forma individual, se dividieron las geometrías optimizadas en cinco clases a partir del valor de $\mathrm{CV}_{\text {ST }}$ y se seleccionaron aquellas que tenían los valores $\mathrm{CV}_{\text {ST }}$ más bajos para que se clasificaran en la primera clase (Jenks, 1967). A medida que las áreas se agruparon (Figura 69), se pudo realizar una unión geométrica de todas ellas, seguido de la creación de un cuadro delimitador o bounding box (Figura 70). Este bounding box permitió discretizar el área de estudio sobre Libya-4.

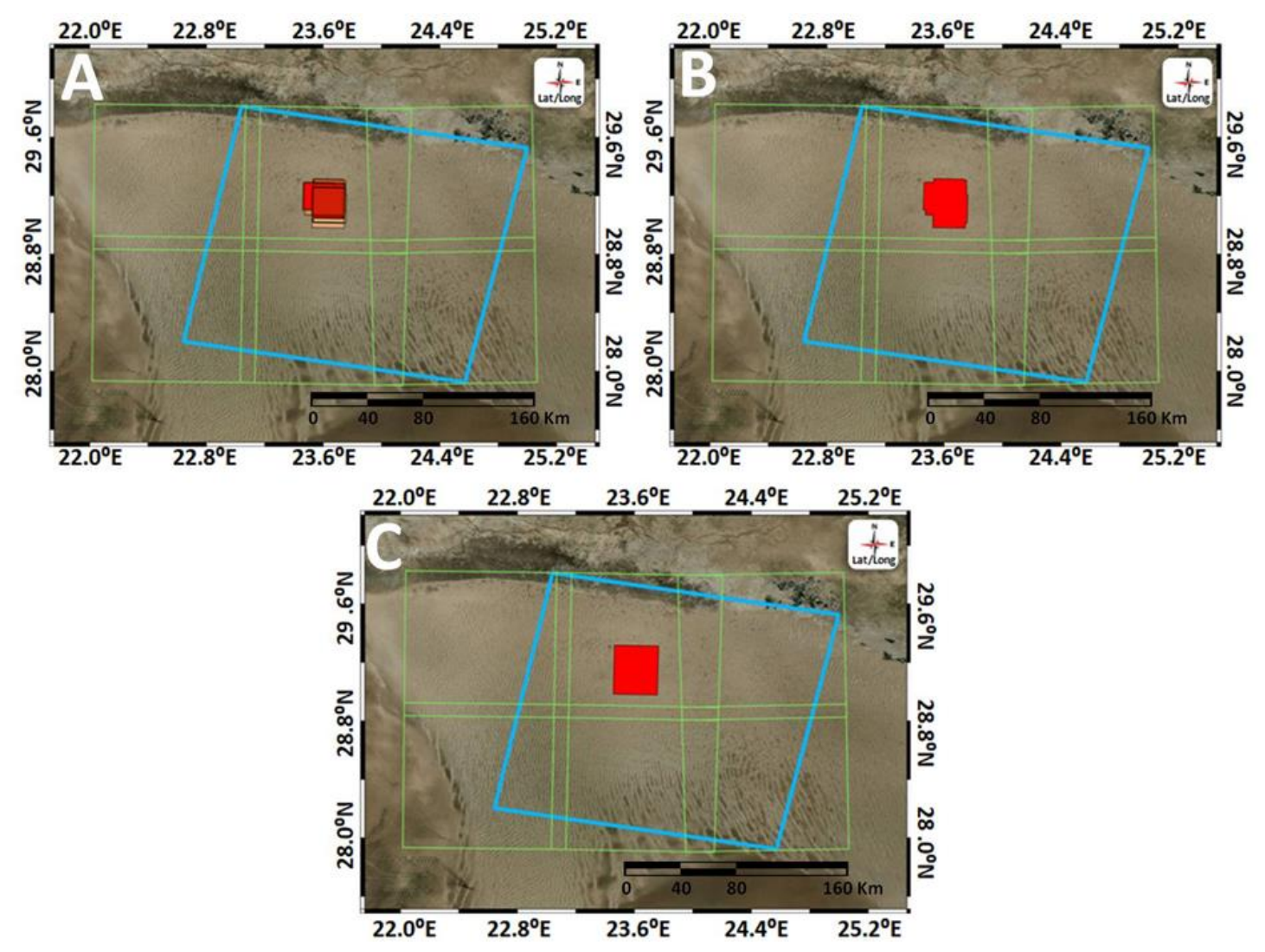

Figura 70. (A) Selección de los mejores AOI para cada banda y sensor. (B) Unión geométrica. (C) Bounding box.

Una vez acotada la zona de estudio de Libya-4 mediante el bounding box, el cual contenía las mejores áreas identificadas para cada banda espectral y sensor, se pudo aplicar la brute-force gracias al tamaño relativamente pequeño del mismo. La bruteforce examina cada posibilidad y selecciona la mejor (Verma et al., 2014; Sen \& Kumar, 2019). Para ello, se realizó el cálculo en el bounding box del CV $\mathrm{V}_{S T}$ mediante brute-force en todas las bandas de manera simultánea, utilizando una ventana móvil cuadrada de tamaño variable y el operador promedio. La ventana fue configurada para moverse en pasos de $30 \mathrm{~m}$ y con un tamaño mínimo de $20 \times 20 \mathrm{~km}^{2}$. Esta operación permitió seleccionar los AOI con el CV $\mathrm{V}_{S T}$ más bajo en todas las bandas y sensores simultáneamente (Figura 71). El uso del operador promedio se consideró el más adecuado, debido a que 
el histograma en el bounding box de las imágenes $\mathrm{CV}_{\text {ST }}$ de las bandas de ambos sensores, presentaba muchas similitudes, no habiendo ninguna banda que pudiera influir significativamente más en la ponderación del cálculo del CV $V_{\text {ST. }}$

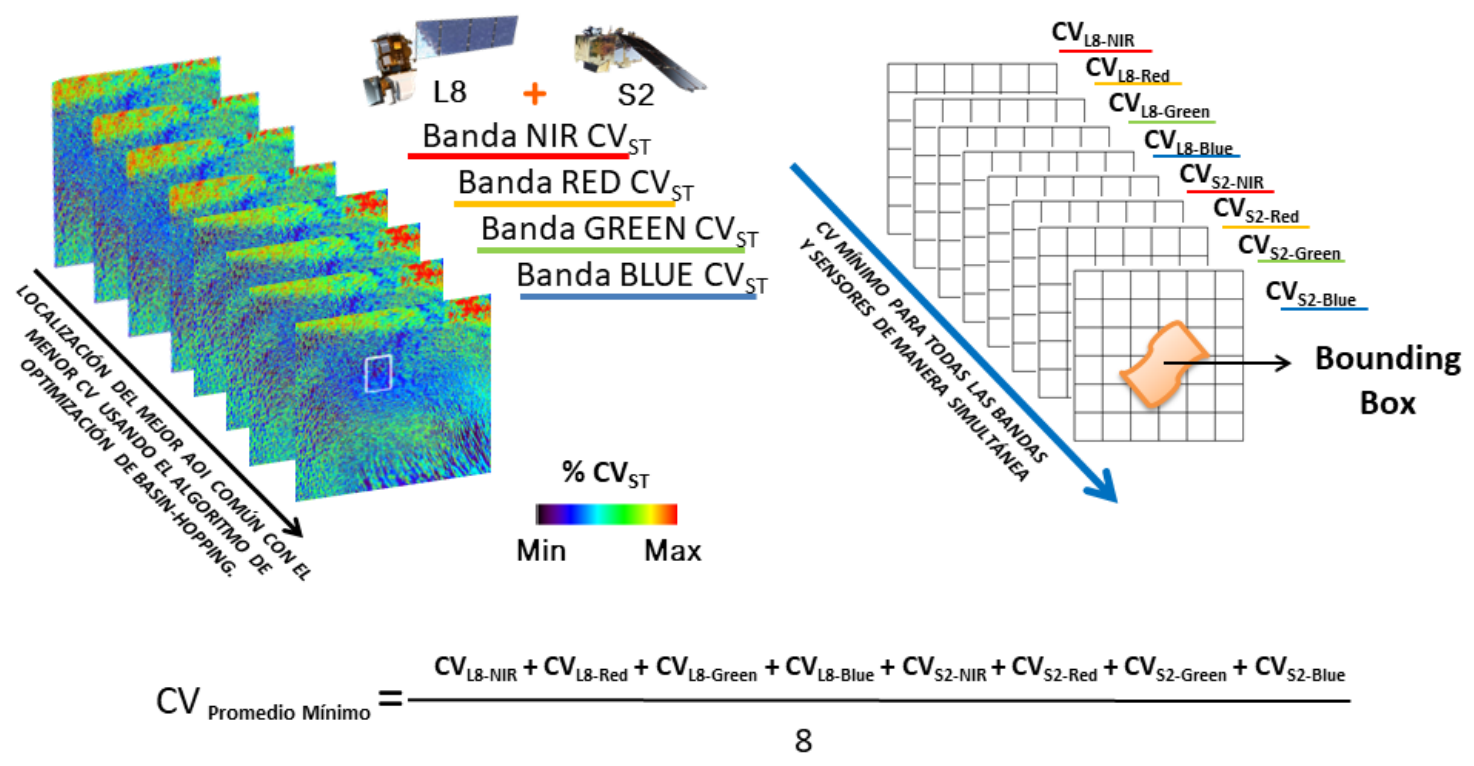

Figura 71. Proceso de localización del OGT dentro del bounding box.

Se puede resumir de forma general el proceso de localización del OGT sobre Libya-4 en las siguientes fases:

$\checkmark$ Selección del data set de reflectancia TOA de L8 y S2.

$\checkmark$ Generación de las imágenes $\mathrm{CV}_{\text {ST }}$ para cada banda.

$\checkmark$ Discretización de la zona de estudio mediante BH-GOT y quasi-Newton Optimization.

$\checkmark$ Generación del bounding box.

$\checkmark$ Cálculo en el bounding box del mínimo $\mathrm{CV}_{\mathrm{ST}}$ mediante brute-force en todas las bandas de manera simultánea.

$\checkmark$ Localización del OGT.

\subsection{Resultados}

En este apartado, se muestran los resultados numéricos y representación gráfica del estudio de uniformidad espacio-temporal sobre Libya-4.

La homogeneidad espacio-temporal de Libya- 4 fue analizada a partir del coeficiente $\mathrm{CV}_{\text {ST }}$ definido en la Ecuación 9. Para cada banda RGBNIR de L8-OLI y S2-MSI se generó su imagen $C_{\text {ST. }}$ En la Figura 72 y Figura 73 , se muestran las imágenes $C_{\text {ST }}$ para cada una de las bandas de ambos sensores mostrando los valores $\mathrm{CV}_{\mathrm{ST}}$ mínimo y máximo en una escala de colores gradual. Conviene recordar que a menor valor de $\mathrm{CV}_{\text {ST }}$ mayor uniformidad espacio-temporal y viceversa. 
De forma general, independientemente de la banda o sensor, las imágenes CV $_{S T}$ presentaban un mismo patrón de homogeneidad. Las zonas más occidentales y situadas cerca del centro de las imágenes presentaban un $\mathrm{CV}_{S T}$ menor, es decir una mayor homogeneidad espacio-temporal. Sin embargo, Las zonas situadas al norte de Libya-4 presentaban valores más elevados de $\mathrm{CV}_{\mathrm{ST}}$, por tanto eran zonas menos homogéneas.

En términos cuantitativos, el rango de valores medios del $\mathrm{CV}_{S T}$ para el caso de L8-OLI oscila entre el $3.147 \%$ de la banda blue y el $3.641 \%$ de la banda red. Para el caso de S2 A/B-MSI los valores medios del $\mathrm{CV}_{\text {ST }}$ oscilan en el rango de $3.734 \%$ de la banda blue a $4.210 \%$ de la banda red. Para ambos satélites, el $\mathrm{CV}_{\mathrm{ST}}$ promedio más bajo y la $\sigma$ menor correspondieron a la banda blue, mientras que el valor $\mathrm{CV}_{\mathrm{ST}}$ promedio más alto y la $\sigma$ más elevada con la banda red. En la Tabla 17 se muestran los valores de $\mathrm{CV}_{\text {ST }}$ promedio para cada banda y sensor.
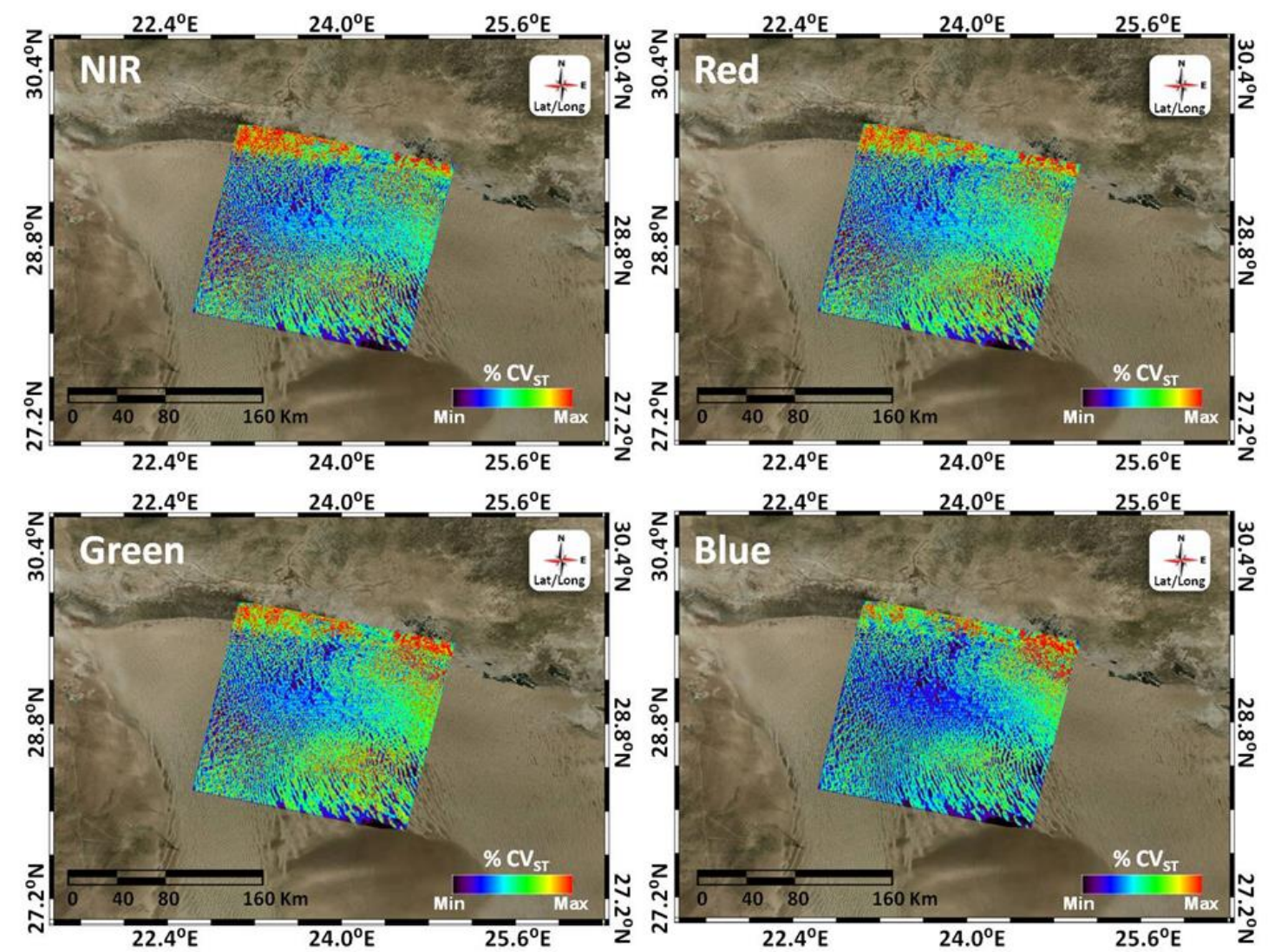

Figura 72. CV $V_{S T}$ para las Bandas RGBNIR de L8-OLI en Libya-4. 

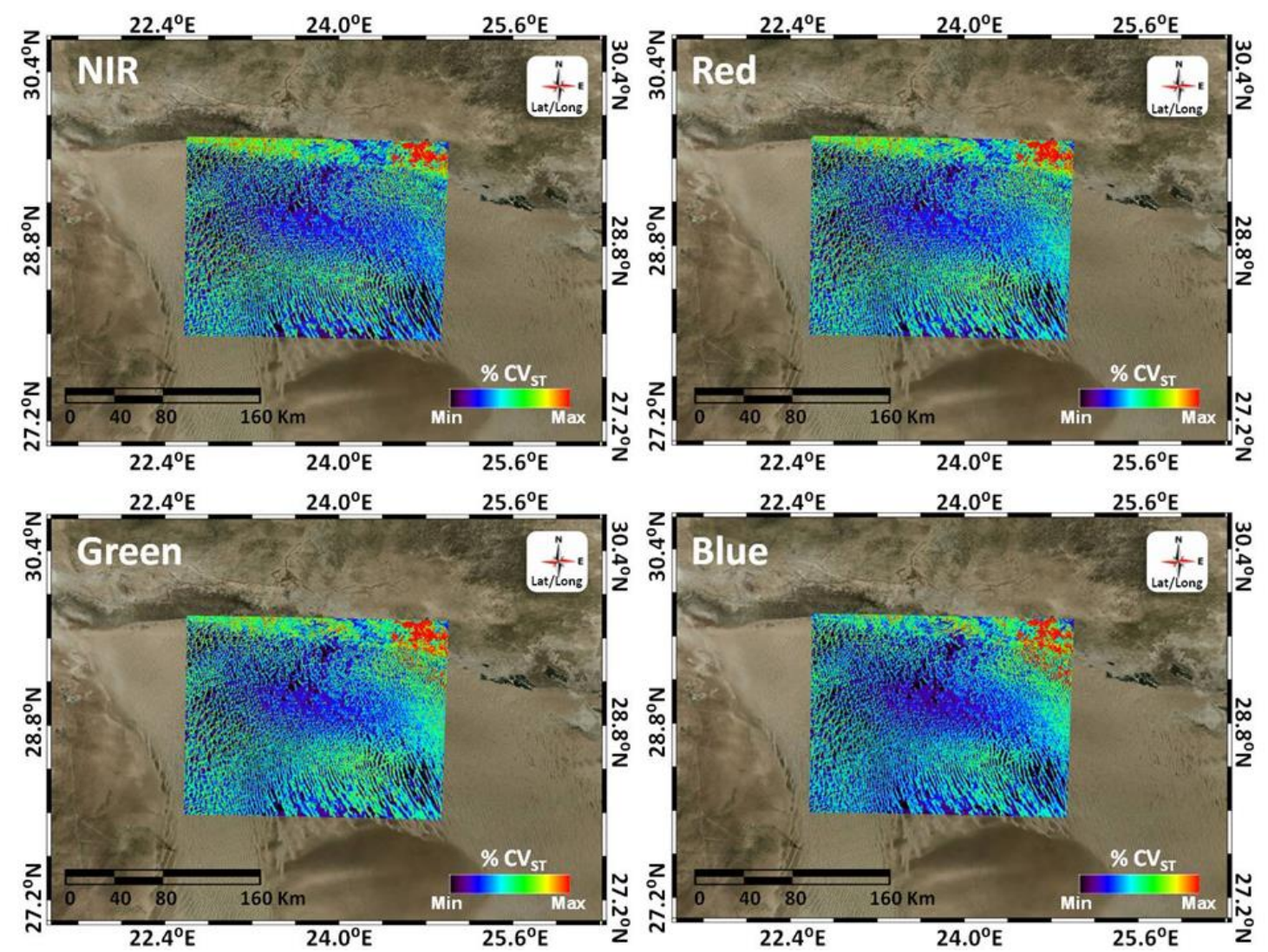

Figura 73. $C V_{\text {St }}$ para las Bandas RGBNIR de S2 A/B-MSI en Libya-4.

Tabla 17. Valores de $\mathrm{CV}_{S T}$ promedios y $\boldsymbol{\sigma}$ para las bandas RGBNIR de los sensores L8 OLI y S2 A/B.

\begin{tabular}{cccc}
\hline & Banda & $\begin{array}{c}\mathbf{C V}_{\text {ST }} \\
\text { Promedio } \\
\text { (\%) }\end{array}$ & $\begin{array}{c}\boldsymbol{C} \text { (\%) } \\
\text { (\%) }\end{array}$ \\
\hline \multirow{4}{*}{ L8 } & NIR & 3.576 & 1.808 \\
& Red & 3.641 & 1.725 \\
& Green & 3.632 & 1.731 \\
& Blue & 3.147 & 1.661 \\
& & & \\
& NIR & 4.184 & 2.552 \\
& Red & 4.210 & 2.360 \\
& Green & 4.114 & 2.139 \\
& Blue & 3.734 & 1.965 \\
\hline
\end{tabular}

Una vez generadas las imágenes $\mathrm{CV}_{S T}$ se procedió a la discretización de la zona de estudio mediante BH-GOT y quasi-Newton Optimization. Cabe recordar que el tamaño de las AOI estaba limitado a $20 \times 20 \mathrm{Km}^{2}$ para evitar el efecto duna y a un máximo de $100 \times 100$ $\mathrm{km}^{2}$. Como se aprecia en la Figura 74 y Figura 75, las AOI encontradas para cada banda y sensor se localizan en la misma zona de Libya-4, solapándose gran parte de los AOI entre sí. Cuanto menor sea la transparencia del color, menor será el $\mathrm{CV}_{\text {ST }}$ promedio de 
la AOI. El tamaño de los AOI optimizados no presentaba elevadas diferencias entre ellos. Este resultado permitió delimitar de una forma clara el AOI a estudio.

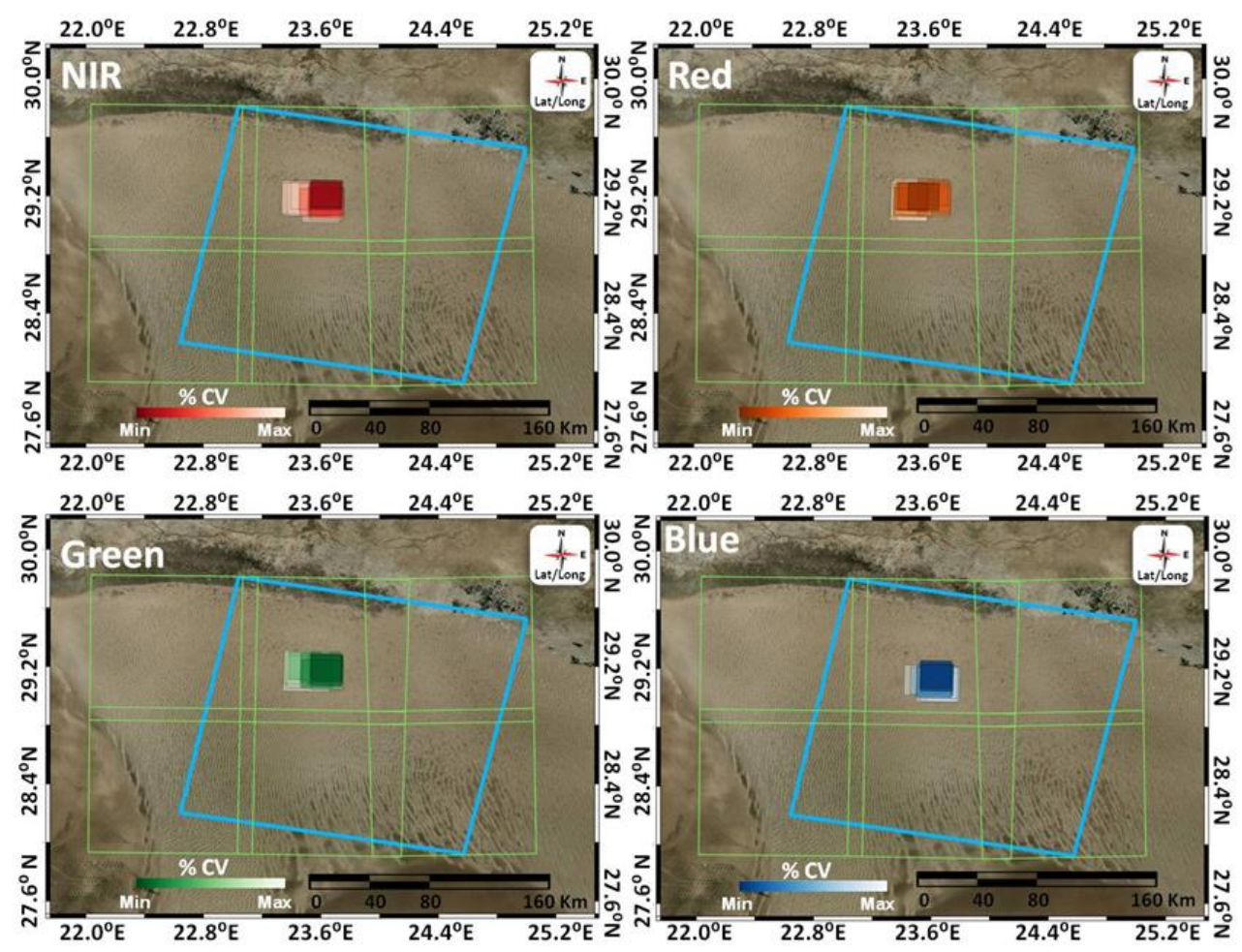

Figura 74. Localización de los AOI optimizados con menos $C V$ ST para las Bandas RGBNIR de L8-OLI en Libya-4.

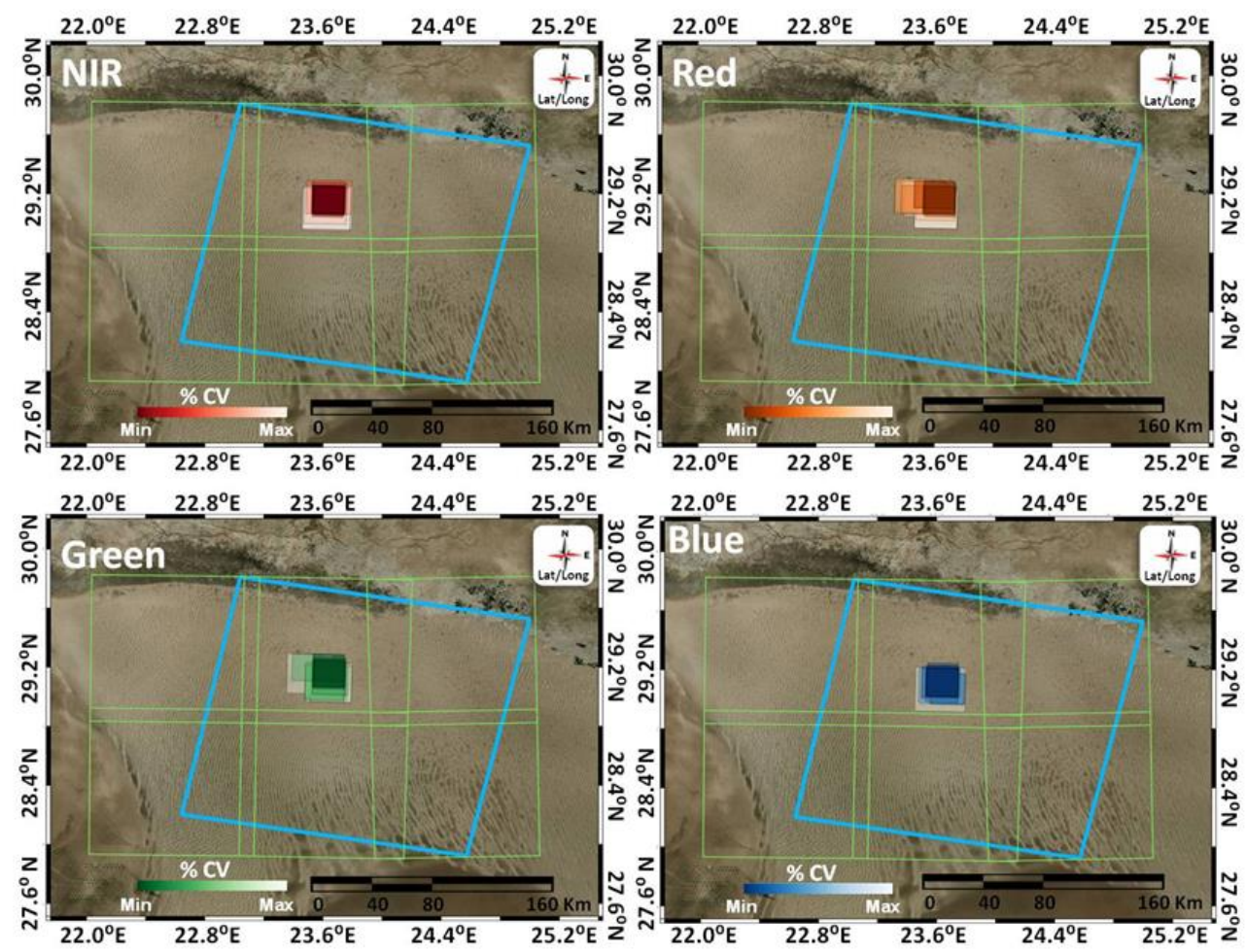

Figura 75. Localización de los AOI optimizados con menos $C V_{S T}$ para las Bandas RGBNIR de S2 A/B - MSI en Libya-4. 
Al igual que con los resultados de las imágenes $\mathrm{CV}_{\mathrm{ST}}$, se presentan los resultados de forma cuantitativa de los valores de $\mathrm{CV}_{\text {ST }}$ promedio y de $\sigma$, así como del tamaño del área para cada uno de los AOI optimizadas. Para facilitar la visualización de los datos, solo se presentan en la Tabla 18 y Tabla 19 los resultados de los 5 mejores AOI optimizadas para cada banda y sensor.

Tabla 18. Valores de $\mathrm{CV}_{\text {ST }}$ promedios y $\sigma$ de los 5 mejores $\mathrm{AOI}$ optimizado para las bandas RGBNIR de los sensores L8-OLI y S2 A/B.

\begin{tabular}{|c|c|c|c|c|c|c|c|c|c|}
\hline & AOI & $\begin{array}{c}C V_{\text {ST }} \\
\text { Promedio } \\
\text { (\%) } \\
\text { Banda } \\
\text { NIR }\end{array}$ & $\begin{array}{c}C V_{S T} \\
\sigma(\%) \\
\text { Banda } \\
\text { NIR }\end{array}$ & $\begin{array}{c}C V_{S T} \\
\text { Promedio } \\
(\%) \\
\text { Banda } \\
\text { Red }\end{array}$ & $\begin{array}{c}C V_{S T} \\
\sigma(\%) \\
\text { Banda } \\
\text { Red }\end{array}$ & $\begin{array}{c}C V_{\text {sT }} \\
\text { Promedio } \\
\text { (\%) } \\
\text { Banda } \\
\text { Green }\end{array}$ & $\begin{array}{c}C V_{\text {ST }} \\
\sigma(\%) \\
\text { Banda } \\
\text { Green }\end{array}$ & $\begin{array}{c}C V_{S T} \\
\text { Promedio } \\
\text { (\%) } \\
\text { Banda } \\
\text { Blue }\end{array}$ & $\begin{array}{c}C V_{\text {ST }} \\
\sigma(\%) \\
\text { Banda } \\
\text { Blue }\end{array}$ \\
\hline \multirow{3}{*}{ L8 } & 1 & 2.3510 & 1.1615 & 2.4431 & 1.0869 & 2.2977 & 1.0346 & 1.8308 & 0.7565 \\
\hline & 4 & 2.3681 & 1.1661 & 2.4535 & 1.0318 & 2.3106 & 1.0722 & 1.8361 & 0.8089 \\
\hline & 5 & 2.3753 & 1.0799 & 2.4555 & 1.1019 & 2.3164 & 0.9422 & 1.8362 & 0.8332 \\
\hline \multirow[b]{3}{*}{ S2 } & 1 & 2.6760 & 1.2922 & 2.7319 & 1.2942 & 2.5270 & 1.1236 & 2.1563 & 0.8760 \\
\hline & 2 & 2.6790 & 1.3244 & 2.7342 & 1.2216 & 2.5274 & 1.1132 & 2.1563 & 0.8793 \\
\hline & 3 & 2.6799 & 1.3185 & 2.7347 & 1.1861 & 2.5299 & 1.1326 & 2.1575 & 0.9828 \\
\hline
\end{tabular}

Tabla 19. Valores de área de los 5 mejores AOI optimizadas para las bandas RGBNIR de los sensores L8-OLI y S2 A/B.

\begin{tabular}{|c|c|c|c|c|c|}
\hline & AOI & $\begin{array}{c}\text { Área km² } \\
\text { (Ellipsoidal, } \\
\text { WGS84) } \\
\text { Banda NIR }\end{array}$ & $\begin{array}{c}\text { Área km² } \\
\text { (Ellipsoidal, } \\
\text { WGS84) } \\
\text { Banda Red }\end{array}$ & $\begin{array}{c}\text { Área km² } \\
\text { (Ellipsoidal, } \\
\text { WGS84) } \\
\text { Banda Green }\end{array}$ & $\begin{array}{c}\text { Área km² }^{2} \\
\text { (Ellipsoidal, } \\
\text { WGS84) } \\
\text { Banda Blue }\end{array}$ \\
\hline \multirow{5}{*}{ L8 } & 1 & 423.693 & 401.722 & 416.296 & 408.967 \\
\hline & 2 & 404.103 & 416.332 & 406.534 & 413.851 \\
\hline & 3 & 416.300 & 409.028 & 411.396 & 428.676 \\
\hline & 4 & 446.283 & 433.745 & 404.106 & 411.407 \\
\hline & 5 & 456.528 & 413.842 & 406.564 & 406.535 \\
\hline \multirow{5}{*}{ S2 } & 1 & 422.050 & 408.149 & 413.845 & 438.705 \\
\hline & 2 & 403.300 & 406.536 & 403.303 & 445.454 \\
\hline & 3 & 425.354 & 408.151 & 437.021 & 402.498 \\
\hline & 4 & 422.875 & 429.506 & 405.719 & 417.120 \\
\hline & 5 & 435.345 & 413.844 & 431.163 & 416.306 \\
\hline
\end{tabular}

Una vez seleccionados los AOls optimizados de cada banda y sensor, se procedió a generar el bounding box a partir de una clasificación de cinco clases sobre los valores de $\mathrm{CV}_{\text {ST }}$ promedios más bajos. Se consideraron únicamente los $\mathrm{AOI}$ que estaban en la primera clase de cada banda, despreciando el resto de AOI y outliers. En la Tabla 20 se especifican las muestras utilizadas para la generación del bounding box. 
Tabla 20. Número de AOI optimizados para cada banda RGBNIR de los sensores L8-OLI y S2 A/B utilizados para la generación del bounding box.

\begin{tabular}{ccccc}
\hline & $\begin{array}{c}\text { Número de AOI } \\
\text { Banda NIR }\end{array}$ & $\begin{array}{c}\text { Número de AOI } \\
\text { Banda Red }\end{array}$ & $\begin{array}{c}\text { Número de AOI } \\
\text { Banda Green }\end{array}$ & $\begin{array}{c}\text { Número de AOI } \\
\text { Banda Blue }\end{array}$ \\
\hline L8 & 4 & $\begin{array}{c}3 \text { (2 Outliers } \\
\text { eliminados }\end{array}$ & 6 & 6 \\
\hline S2 & 13 & 8 & 6 & 12 \\
\hline
\end{tabular}

Una vez discretizada la zona de estudio a partir del bounding box, se procedió a aplicar sobre esa zona el brute-force algorithm. Este algoritmo analizó todas las posibilidades existentes mediante una ventana móvil con un paso de un pixel L8 y una longitud de lado de $30 \mathrm{~m}$. Hay que tener en cuenta que el brute-force algorithm contaba con tres grados de libertad: $\mathrm{CV}_{\text {ST }}$ promedio mínimo, área cuadrada variable y coordenadas espaciales. Además, las operaciones del brute-force algorithm contaban con dos restricciones como eran el tamaño mínimo del área variable establecida en $20 \times 20 \mathrm{~km}^{2}$ para evitar el efecto duna (Govaerts, 2015) y una búsqueda restringida al área delimitada por el bounding box.

Los 10 mejores AOIs localizados en Libya-4 presentan similar extensión y localización (Figura 76). Los valores cuantitativos relativos a la $\sigma$, área, tamaño de lado y $\mathrm{CV}_{S T}$ promedio de estos AOIs se muestran en la Tabla 21. La Figura 77 muestra la comparativa entre tamaño del lado del cuadrado de cada AOI localizado y su valor de $\mathrm{CV}_{\text {ST }}$ promedio.

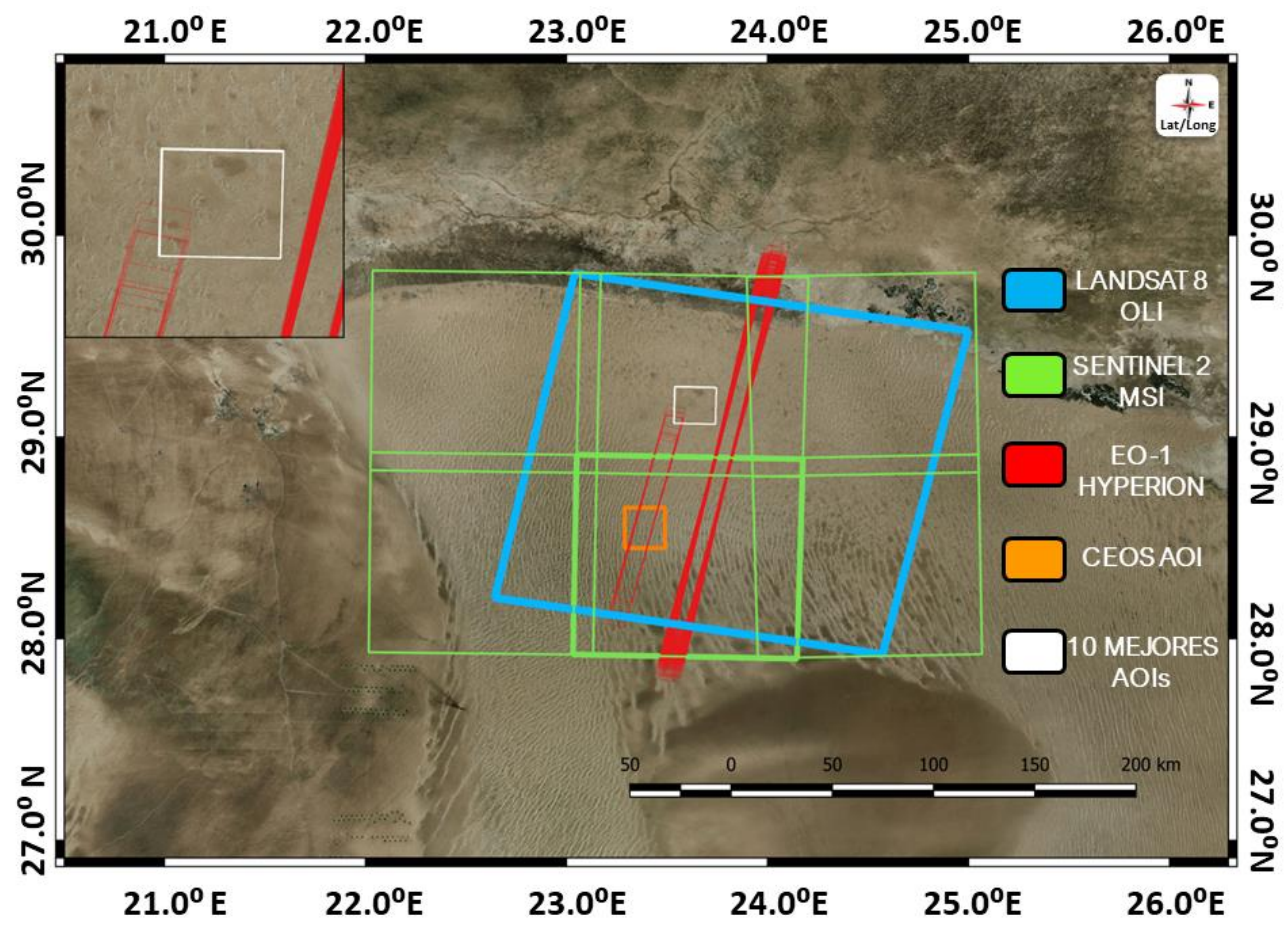

Figura 76. Localización de los 10 mejores AOIs para Libya-4. 
Tabla 21. Características de $\mathrm{CV}_{\mathrm{ST}}$ promedio, $\sigma$, área y tamaño del lado para los 10 mejores AOls encontrados en el bounding box por el brute-force algorithm.

\begin{tabular}{|c|c|c|c|c|c|}
\hline & AOI & $\begin{array}{c}C V_{\text {ST }} \text { Promedio } \\
\text { (\%) }\end{array}$ & $\begin{array}{c}C V_{\text {ST }} \sigma \\
(\%)\end{array}$ & $\begin{array}{c}\text { Área km² } \\
\text { (Elipsoidal, } \\
\text { WGS84) }\end{array}$ & $\begin{array}{c}\text { Tamaño de } \\
\text { lado del AOI } \\
(\mathbf{k m})\end{array}$ \\
\hline & 1 & 2.3778 & 1.0368 & 400.000 & 20.000 \\
\hline & 2 & 2.3779 & 1.0366 & 401.201 & 20.030 \\
\hline & 3 & 2.3780 & 1.0367 & 402.404 & 20.060 \\
\hline & 4 & 2.3782 & 1.0368 & 403.608 & 20.090 \\
\hline AOls & 5 & 2.3783 & 1.0358 & 404.814 & 20.120 \\
\hline \multirow{5}{*}{ L8 \& S2 } & 6 & 2.3785 & 1.0360 & 406.023 & 20.150 \\
\hline & 7 & 2.3786 & 1.0377 & 407.232 & 20.180 \\
\hline & 8 & 2.3787 & 1.0376 & 408.444 & 20.210 \\
\hline & 9 & 2.3789 & 1.0378 & 409.658 & 20.240 \\
\hline & 10 & 2.3790 & 1.0372 & 410.873 & 20.270 \\
\hline
\end{tabular}

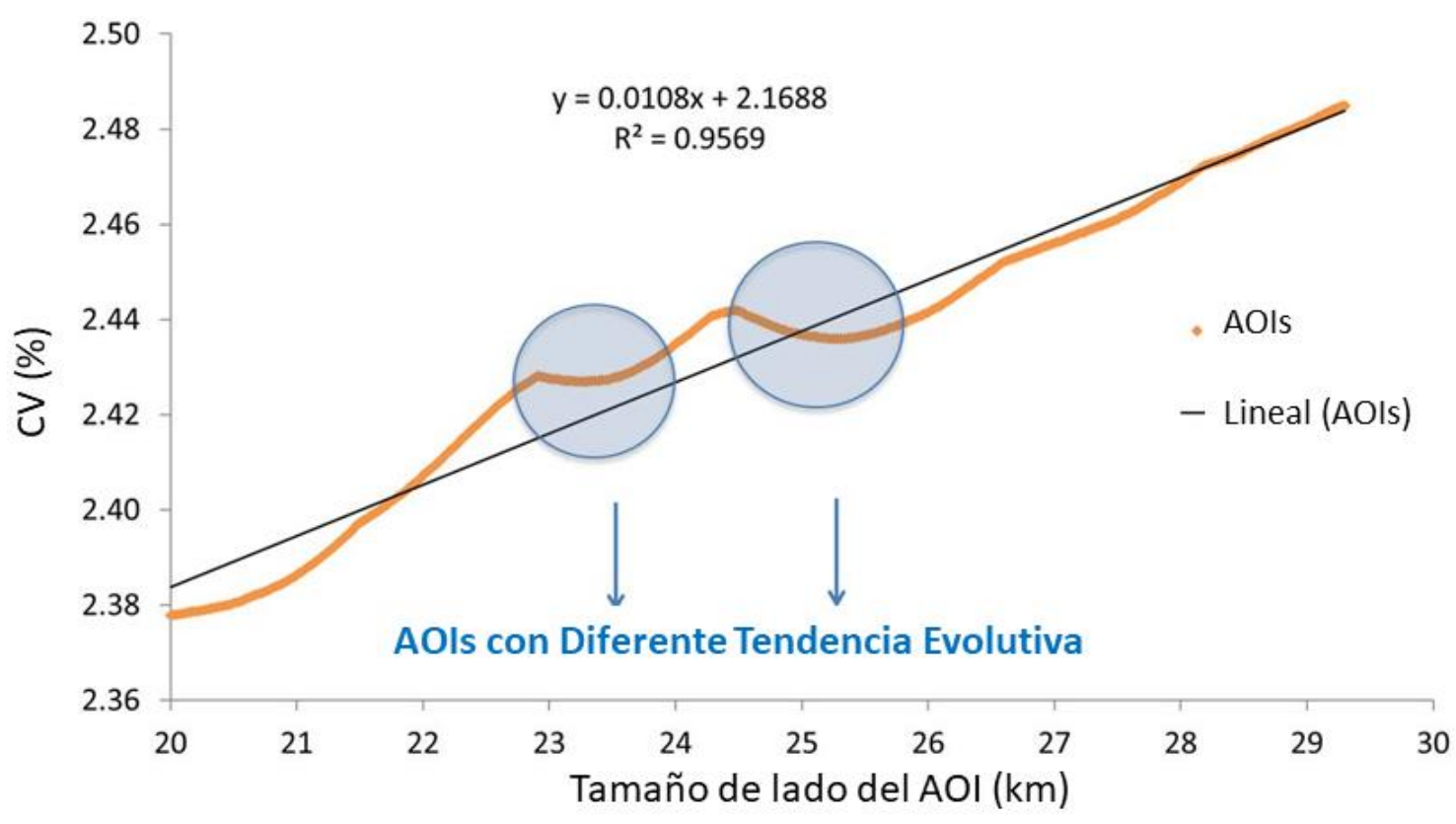

Figura 77. Relación lineal entre el valor de CVST y el tamaño de lado de los AOls localizados.

Una vez encontrados y caracterizados los 10 mejores AOls posibles, se procedió a seleccionar el target de calibración que se ha definido como OGT. Este OGT era el que menor $\mathrm{CV}_{\text {ST }}$ promedio tenía entre el data set de AOI común para L8 y S2 encontrados. Para la validación de los resultados obtenidos en el OGT, se realizó una comparativa de los mismos con el target sugerido por CEOS definido previamente en esta tesis como CEOS AOI. Conviene recordar que el tamaño del mismo es de $20 \times 20 \mathrm{~km}^{2}$ al igual que el OGT y se ubica en el tile 34RGS de S2 (Figura 76). Esta comparativa entre los valores de $\mathrm{CV}_{\text {ST }}$ obtenido en el OGT y CEOS AOI permitió ponderar el nivel de homogeneidad de la nueva área optimizada. 
En la Tabla 22 se presenta la localización del área del OGT. En la Tabla 23 se muestra la comparativa del CV $\mathrm{V}_{\text {ST }}$ promedio entre el CEOS AOI y el OGT para cada banda y sensor.

Tabla 22. Coordenadas de las esquinas del OGT en la proyección UTM 34 N, WGS84.

\begin{tabular}{ccc}
\hline OGT Esquina & Este $(\mathbf{m})$ & Norte $(\mathbf{m})$ \\
\hline Inferior izquierda & 746850 & 3218250 \\
\hline Inferior derecha & 766860 & 3218250 \\
\hline Superior derecha & 766860 & 3238230 \\
\hline Superior izquierda & 746850 & 3238230 \\
\hline
\end{tabular}

Tabla 23. Comparativa de los resultados de $\mathrm{CV}_{\text {ST }}$ para las bandas de L8 y S2 sobre el OGT y la zona CEOS.

\begin{tabular}{|c|c|c|c|c|}
\hline & $\begin{array}{c}\text { Banda NIR } \\
\text { CV }\end{array}$ & $\begin{array}{c}\text { Banda Red } \\
\text { CV }\end{array}$ & $\begin{array}{c}\text { Banda Green } \\
\text { CV } \\
\text { ST (\%) }\end{array}$ & $\begin{array}{c}\text { Banda Blue } \\
\text { CV } \\
\end{array}$ \\
\hline $\begin{array}{c}\text { Zona CEOS } \\
\text { L8 }\end{array}$ & 3.5151 & 3.3724 & 3.2194 & 2.7248 \\
\hline $\begin{array}{l}\text { OGT } \\
\text { L8 }\end{array}$ & 2.3561 & 2.4539 & 2.3047 & 1.8308 \\
\hline $\begin{array}{l}\text { Zona CEOS } \\
\quad \text { S2 }\end{array}$ & 4.1590 & 3.9481 & 3.6733 & 3.2507 \\
\hline $\begin{array}{c}\text { OGT } \\
\text { S2 }\end{array}$ & 2.6698 & 2.7297 & 2.5192 & 2.1585 \\
\hline
\end{tabular}

\subsection{Discusión}

Tras el lanzamiento de S2 B, se han multiplicado el número de datos disponibles de EO. La misión S2 y L8 de forma conjunta ponen a disposición de la comunidad científica un volumen de información de alta calidad imprescindible para el estudio global del planeta Tierra. Utilizando como ventaja esta disponibilidad de datos sobre cualquier punto de la superficie terrestre, se ha hecho uso de datos L8-OLI y S2 A/B-MSI con el principal objetivo de la localización de un OGT dentro de Libya-4 que cumpla con el mayor nivel de homogeneidad espacio-temporal. Para ello, se ha utilizado una larga serie temporal de datos de ambos sensores y se ha combinado con una novedosa metodología sustentada en la combinación de un algoritmo quasi-Newton (Battiti \& Masulli, 1990), 
una técnica BH-GOT (Iwamatsu \& Okabe, 2004) y la aplicación del algoritmo de bruteforce (Verma et al., 2014; Sen \& Kumar, 2019). La definición de un coeficiente (CV $\mathrm{V}_{\text {ST }}$ ) que permitiese definir la homogeneidad espacio-temporal junto con la implementación de estas técnicas de optimización han permitió definir y localizar un área de calibración en Libya-4 denominado OGT, con unas características del orden de $1.5 \%$ veces mayor homogeneidad espacio-temporal (Tabla 23), con respecto al CEOS AOI. Aunque en este estudio no se ha considerado factor determinante en la elección del área optimizado la disponibilidad de datos EO-1 Hyperion que facilitan la caracterización de la superficie (USGS, 2020d), es importante resaltar la presencia de adquisiciones de este sensor hiperespectral en el OGT (Figura 76).

Las imágenes de $\mathrm{CV}_{\text {ST }}$ generadas para las bandas RGBNIR de L8 y S2 (Figura 72 y Figura 73) presentan un patrón de $\mathrm{CV}_{\text {ST }}$ muy similar. Las zonas con mayor y menor $\mathrm{CV}_{\text {ST }}$ se encuentran localizadas prácticamente en las mismas áreas. Esta característica refuerza y valida el buen funcionamiento del coeficiente $\mathrm{CV}_{\mathrm{ST}}$ (Ecuación 9) definido como métrica de la homogeneidad espacio-temporal.

Los resultados obtenidos en la discretización de la zona de estudio a partir de los AOI optimizados para todas las bandas y sensores utilizados (Figura 74 y Figura 75), marcan con precisión esa área como la más estable dentro de Libya-4. Una consecuencia de la elevada homogeneidad del bounding box (Figura 70) son los resultados de los 10 mejores AOls obtenidos tras la aplicación del algoritmo de brute-force, los cuales prácticamente se solapan (Figura 76).

Se observa un nivel más elevado de incertidumbre en los valores de $\mathrm{CV}_{S T}$ de L8 para la zona CEOS AOI con respecto a los valores de S2 en la misma zona, siendo esas diferencias entre las mismas bandas de diferentes sensores, de forma general, superiores al $0.5 \%$ (Tabla 23). En cambio, en el OGT las diferencias de los valores de $\mathrm{CV}_{\text {ST }}$ entre las mismas bandas de los sensores L8 y S2 son menores y prácticamente constantes en todas las bandas, manteniéndose del orden del $0.3 \%$ (Tabla 23). Esta diferencia entre bandas de ambos sensores, mucho menor y más constante en el OGT que en el CEOS AOI, pone de manifiesto la elevada homogeneidad espacio-tempo de la nueva área optimizada. Para ambas zonas, CEOS AOI y OGT, los valores de CV $\mathrm{ST}_{\text {T }}$ son más elevados en S2 que en L8, probablemente debido a la mayor resolución de S2 (Tabla 23).

Resulta interesante también señalar, la relación lineal existente entre $\mathrm{CV}_{S T}$ y el tamaño del lado del cuadrado de las AOI encontradas (Figura 77), así como la presencia de dos zonas diferenciadas donde esta relación en vez de aumentar de forma lineal, disminuye o permanece constante. Estas dos zonas pueden ser consecuencia de la aplicación del algoritmo de brute-force al localizar de forma puntual, píxeles con valores de CV $\mathrm{CV}_{\text {ST }}$ muy dispares. A pesar de esta relación lineal, el crecimiento del valor de $\mathrm{CV}_{\text {ST }}$ con respecto al tamaño de lado del cuadrado es mucho menor. Un ejemplo de este comportamiento, es que mientras el OGT presenta un valor de $\mathrm{CV}_{\text {ST }}$ en el área común de L8 y S2 de $2.377 \%$ 
(Tabla 21) y un área cuadrada de $20 \mathrm{~km}$ de lado, el peor AOI localizado por algoritmo de brute-force muestra un valor $\mathrm{CV}_{\text {ST }}$ de $2.485 \%$ y una longitud lateral del cuadrado 29.3 $\mathrm{km}$ que es exactamente la longitud del lado del bounding box. Estos resultados confirman la poca variabilidad espacio-temporal tanto del OGT y del bounding box.

Como se ha comentado previamente, la disponibilidad de datos hiperespectral del sensor Hyperion a bordo del satélite EO-1 en el OGT, permiten caracterizar aún más esta zona optimizada. Esta presencia de datos hiperespectrales puede ser una ventaja en la cross-calibration entre sensores con grandes diferencias en su RSRs que hace necesario la aplicación de un factor de ajuste de banda (SBAF) (Chander et al., 2012).

\subsection{Conclusiones}

En este capítulo, se presenta una metodología de estudio de la homogeneidad temporal y espacial para el caso particular de Libya-4 a partir de datos L8-OLI y S2 A/B-MSI. Las imágenes de $\mathrm{CV}_{\text {ST }}$ generadas muestran una elevada similitud independientemente de la banda y el sensor elegido. Este comportamiento indica que el coeficiente $\mathrm{CV}_{S T}$ definido en esta metodología es una buena métrica de la homogeneidad espacio-temporal. Esta conclusión queda aún más reforzada después del proceso de optimización y delimitación del bounding box, así como, tras los resultados del algoritmo de brute-force donde el baricentro de las mejores áreas cuadradas encontradas, se localizan muy próximos unos

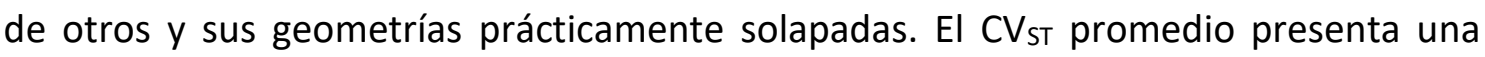
menor incertidumbre a medida que se reduce la superficie. Prueba de ello, fueron los valores de numerosas AOI localizadas por el algoritmo brute-force, cuya superficie estaba cerca del mínimo de $20 \times 20 \mathrm{~km}^{2}$ establecido para evitar el efecto de duna.

La comparativa de resultados entre el CEOS AOI y el OGT, muestra la gran homogeneidad de este último, con valores de $\mathrm{CV}_{\text {ST }}$ en el rango de $1.83 \%-2.73 \%$ frente a valores del CEOS AOI en el rango de $2.73 \%$ - 4.16\%. Ambas zonas de calibración presentan la misma superficie, exactamente de $20 \times 20 \mathrm{~km}^{2}$. Estos resultados validan la utilización del OGT para la cross-calibration entre sensores dentro de Libya-4.

Esta metodología se considera apropiada no solo para su aplicación en otros PICS y los Extended Pseudo Invariant Calibration Sites - EPICS, sino que podría extenderse también a la localización previa de zonas óptimas de medida para la calibración vicaria.

\subsection{Referencias}

Battiti, R., \& Masulli, F. (1990). BFGS optimization for faster and automated supervised learning. In International neural network conference (pp. 757-760). Springer, Dordrecht.

Becker-Reshef, I., Justice, C., Sullivan, M., Vermote, E., Tucker, C., Anyamba, A., ... \& Hansen, M. (2010). Monitoring global croplands with coarse resolution earth 
observations: The Global Agriculture Monitoring (GLAM) project. Remote Sensing, 2(6), 1589-1609.

Berthelot, B. (2015). Prototyping a vicarious calibration system for GEOSS in the frame of the CEOS. Technical Note-Methodology Used to Identify a Site, ACTION-TN010-MAG.

Berthelot, B., \& Henry, P. (2017). Radiometric Calibration Workshop for European Missions.https://earth.esa.int/documents/700255/3194632/B\%2B\%C2\%AEatri ce+Berthelot PICSCAR-PPT-022-

MAGv1.0 BBerthelot PHenry 20170830.pdf/9ddad0e8-532c-4f6c-9f19-

404c8280755c (Accedido el 24 Mayo de 2020)

Bhatt, R., Doelling, D. R., Wu, A., Xiong, X., Scarino, B. R., Haney, C. O., \& Gopalan, A. (2014). Initial stability assessment of S-NPP VIIRS reflective solar band calibration using invariant desert and deep convective cloud targets. Remote Sensing, 6(4), 2809-2826.

Broyden, C.G. (1970). The convergence of a class of double-rank minimization algorithms 1. General considerations. IMa J appl Math, 6(1), 76-90.

Chander, G., Christopherson, J. B., Stensaas, G. L., \& Teillet, P. M. (2007). Online catalog of world-wide test sites for the Post-Lanzamiento characterization and calibration of optical sensors. In 58th International Astronautical Congress 2007 (Vol. 3, pp. 2043-2051).

Chander, G., Haque, M. O., Sampath, A., Brunn, A., Trosset, G., Hoffmann, D., D.; Roloff, S., Thiele, M., \& Anderson, C. (2013). Radiometric and geometric assessment of data from the RapidEye constellation of satellites. International Journal of Remote Sensing, 34(16), 5905-5925.

Chander, G., Mishra, N., Helder, D. L., Aaron, D. B., Angal, A., Choi, T., ... \& Doelling, D. R. (2012). Applications of spectral band adjustment factors (SBAF) for crosscalibration. IEEE Transactions on Geoscience and Remote Sensing, 51(3), 12671281.

Chander, G., Mishra, N., Helder, D. L., Aaron, D. B., Angal, A., Choi, T., ... \& Doelling, D. R. (2012). Applications of spectral band adjustment factors (SBAF) for crosscalibration. IEEE Transactions on Geoscience and Remote Sensing, 51(3), 12671281.

Chander, G., Xiong, X. J., Choi, T. J., \& Angal, A. (2010). Monitoring on-orbit calibration stability of the Terra MODIS and Landsat 7 ETM+ sensors using pseudo-invariant test sites. Remote Sensing of Environment, 114(4), 925-939. 
Choi, T. J., Xiong, X., Angal, A., Chander, G., \& Qu, J. J. (2014). Assessment of the spectral stability of Libya 4, Libya 1, and Mauritania 2 sites using Earth Observing One Hyperion. Journal of Applied Remote Sensing, 8(1), 083618.

Coluzzi, R., Imbrenda, V., Lanfredi, M., \& Simoniello, T. (2018). A first assessment of the Sentinel-2 Level 1-C cloud mask product to support informed surface analyses. Remote sensing of environment, 217, 426-443.

Corbane, C., Najman, L., Pecoul, E., Demagistri, L., \& Petit, M. (2010). A complete processing chain for ship detection using optical satellite imagery. International Journal of Remote Sensing, 31(22), 5837-5854

Cosnefroy, H., Leroy, M., \& Briottet, X. (1996). Selection and characterization of Saharan and Arabian desert sites for the calibration of optical satellite sensors. Remote Sensing of Environment, 58(1), 101-114.

De Vries, C., Danaher, T., Denham, R., Scarth, P., \& Phinn, S. (2007). An operational radiometric calibration procedure for the Landsat sensors based on pseudoinvariant target sites. Remote Sensing of Environment, 107(3), 414-429.

Denis, G., Claverie, A., Pasco, X., Darnis, J. P., de Maupeou, B., Lafaye, M., \& Morel, E. (2017). Towards disruptions in Earth observation? New Earth Observation systems and markets evolution: Possible scenarios and impacts. Acta Astronautica, 137, 415-433.

Díaz, R. (2017). Global Optimization on Complex Systems.

Dwyer, J. L., Roy, D. P., Sauer, B., Jenkerson, C. B., Zhang, H. K., \& Lymburner, L. (2018). Analysis ready data: Enabling analysis of the Landsat archive. Remote Sensing, 10(9), 1363.

ESA, (2017). Workshop on Radiometric Calibration for European Optical Missions, Frascati ESA/ESRIN, Italy. https://earth.esa.int/documents/700255/3194632/RadWS MoM Final.pdf/5fd 20f68-60b3-488a-a2bd-4732a3043a21 (Accedido el 24 de Mayo de 2020)

ESA, Sentinel 2. https://sentinel.esa.int/web/sentinel/missions/sentinel-2 (Accecido el 21 de Mayo de 2020)

Fletcher, R. (1970). A new approach to variable metric algorithms. The computer journal, 13(3), 317-322.

Gascon, F., Bouzinac, C., Thépaut, O., Jung, M., Francesconi, B., Louis, J., ... \& Languille, F. (2017). Copernicus Sentinel-2A calibration and products validation status. Remote Sensing, 9(6), 584. 
GEE, Google Earth Engine. https://earthengine.google.com/ (Accecido el 30 de Mayo de 2020)

Gorelick, N., Hancher, M., Dixon, M., Ilyushchenko, S., Thau, D., \& Moore, R. (2017). Google Earth Engine: Planetary-scale geospatial analysis for everyone. Remote sensing of Environment, 202, 18-27.

Govaerts, Y. M. (2015). Sand dune ridge alignment effects on surface BRF over the Libya4 CEOS calibration site. Sensors, 15(2), 3453-3470.

Govaerts, Y. M., Clerici, M., \& Clerbaux, N. (2004). Operational calibration of the Meteosat radiometer VIS band. IEEE Transactions on Geoscience and Remote Sensing, 42(9), 1900-1914.

Gu, X., GUYOT, M., \& Verbrugghe, M. (1990). Analyse de la variabilité spatiale d'un sitetest. Exemple de la Crau (France). Photo interprétation (Paris), 29(1), 39-52

Heidinger, A. K., Cao, C., \& Sullivan, J. T. (2002). Using Moderate Resolution Imaging Spectroradiometer (MODIS) to calibrate advanced very high resolution radiometer reflectance channels. J. Geophys. Res, 107, 4702.

Helder, D. L., Basnet, B., \& Morstad, D. L. (2010). Optimized identification of worldwide radiometric pseudo-invariant calibration sites. Canadian Journal of Remote Sensing, 36(5), 527-539.

Helder, D., Markham, B., Morfitt, R., Storey, J., Barsi, J., Gascon, F., ... \& Lewis, A. (2018). Observations and Recommendations for the Calibration of Landsat $8 \mathrm{OLI}$ and Sentinel 2 MSI for improved data interoperability. Remote Sensing, 10(9), 1340.

Iwamatsu, M., \& Okabe, Y. (2004). Basin hopping with occasional jumping. Chemical Physics Letters, 399(4-6), 396-400.

Jenks, G. F. (1967). The data model concept in statistical mapping. International yearbook of cartography, 7, 186-190.

Jha, M. N., Levy, J., \& Gao, Y. (2008). Advances in remote sensing for oil spill disaster management: state-of-the-art sensors technology for oil spill surveillance. Sensors, 8(1), 236-255.

Kharbouche, S., \& Muller, J. P. (2016). Evaluation of the Main CEOS Pseudo Calibration Sites Using MODIS BRDF/ALBEDO Products. In ISPRS-International Archives of the Photogrammetry, Remote Sensing and Spatial Information Sciences (Vol. 41, pp. 217-220). Copernicus Gesellschaft MBH. 
Kneubühler, M., Schaepman, M. E., Thome, K., \& Danesy, D. (2006). Long-term vicarious calibration efforts of MERIS at railroad valley playa (NV)-An update. ESA-SP, (615), online.

Lacherade, S., Fougnie, B., Henry, P., \& Gamet, P. (2013). Cross calibration over desert sites: Description, methodology, and operational implementation. IEEE Transactions on Geoscience and Remote Sensing, 51(3), 1098-1113.

Li, Z., \& Scheraga, H. A. (1987). Monte Carlo-minimization approach to the multipleminima problem in protein folding. Proceedings of the National Academy of Sciences, 84(19), 6611-6615.

Liu, G., Heron, S. F., Eakin, C. M., Muller-Karger, F. E., Vega-Rodriguez, M., Guild, L. S., ... \& Strong, A. E. (2014). Reef-scale thermal stress monitoring of coral ecosystems: new 5-km global products from NOAA Coral Reef Watch. Remote Sensing, 6(11), 11579-11606.

Lyapustin, A., Wang, Y., Xiong, X., Meister, G., Platnick, S., Levy, R., \& Hall, F. (2014). Scientific impact of MODIS C5 calibration degradation and C6+ improvements. Atmospheric Measurement Techniques, 7(12).

Mhangara, P., \& Mapurisa, W. (2019). Multi-Mission Earth Observation Data Processing System. Sensors, 19(18), 3831.

Mishra, N., Helder, D., Angal, A., Choi, J., \& Xiong, X. (2014). Absolute calibration of optical satellite sensors using Libya 4 pseudo invariant calibration site. Remote sensing, 6(2), 1327-1346.

Mishra, N., Helder, D., Barsi, J., \& Markham, B. (2016). Continuous calibration improvement in solar reflective bands: Landsat 5 through Landsat 8. Remote sensing of environment, 185, 7-15.

NOAA, (2018). Advisory Committee on Commercial Remote Sensing (ACCRES). https://www.nesdis.noaa.gov/CRSRA/pdf/AACRES meeting 2018 Euroconsult. pdf (Accedido el 24 de Mayo de 2020).

Rao, C. R. N., \& Chen, J. (1999). Revised Post-Lanzamiento calibration of the visible and near-infrared channels of the Advanced Very High Resolution Radiometer (AVHRR) on the NOAA-14 spacecraft. International Journal of Remote Sensing, 20(18), 3485-3491.

Roy, D. P., Wulder, M. A., Loveland, T. R., Woodcock, C. E., Allen, R. G., Anderson, M. C., ... \& Scambos, T. A. (2014). Landsat-8: Science and product vision for terrestrial global change research. Remote sensing of Environment, 145, 154-172. 
Sen, S., \& Kumar, A. (2019). Design and Analysis of Algorithms: A Contemporary Perspective. Cambridge University Press.

Shanno, D. F. (1970). Conditioning of quasi-Newton methods for function minimization. Mathematics of computation, 24(111), 647-656.

Sterckx, S., Livens, S., \& Adriaensen, S. (2013). Rayleigh, deep convective clouds, and cross-sensor desert vicarious calibration validation for the PROBA-V mission. IEEE transactions on geoscience and remote sensing, 51(3), 1437-1452.

Sun, L., Mi, X., Wei, J., Wang, J., Tian, X., Yu, H., \& Gan, P. (2017). A cloud detection algorithm-generating method for remote sensing data at visible to short-wave infrared wavelengths. ISPRS journal of photogrammetry and remote sensing, $124,70-88$.

Teillet, P. M., \& Chander, G. (2010). Terrestrial reference standard sites for postlaunch sensor calibration. Canadian Journal of Remote Sensing, 36(5), 437-450.

Tuli, F. T. Z., Pinto, C. T., Angal, A., Xiong, X., \& Helder, D. (2019). New Approach for Temporal Stability Evaluation of Pseudo-Invariant Calibration Sites (PICS). Remote Sensing, 11(12), 1502.

USGS, Earth Observing 1 (EO-1). https://eo1.usgs.gov/ (Accedido el 29 de Mayo de 2020d)

USGS, Earth Test Sites Catalog. https://calval.cr.usgs.gov/apps/test sites catalog (Accedido el 25 de Mayo de 2020a)

USGS, Landsat Collection $1 . \quad$ https://www.usgs.gov/landresources/nli/landsat/landsat-collection-1?qtscience support page related con=1\#qtscience support page related_con (Accedido el 29 de Mayo de 2020e)

USGS, Remote Sensing Technologies. CalVal Portal. https://calval.cr.usgs.gov/apps/libya-4 (Accedido el 29 de Mayo de 2020b)

$\begin{array}{llllll}\text { USGS, } & \text { Version } & 4.0 & \text { Landsat } & 8 & \text { Data }\end{array}$ Handbook. https://www.usgs.gov/media/files/landsat-8-data-users-handbook (Accedido el 29 de Mayo de 2020c)

Verma, D., Kakkar, N., \& Mehan, N. (2014). Comparison of brute-force and KD tree algorithm. International Journal of Advanced Research in Computer and Communication Engineering, 3(1), 5291-5294. 
Vescovi, F. D., Lankester, T., Coleman, E., \& Ottavianelli, G. (2015). Harmonisation initiatives of Copernicus data quality control. The International Archives of Photogrammetry, Remote Sensing and Spatial Information Sciences, 40(7), 713.

Wales, D. (2003). Energy landscapes: Applications to clusters, biomolecules and glasses. Cambridge University Press.

Wales, D. J., \& Doye, J. P. (1997). Global optimization by basin-hopping and the lowest energy structures of Lennard-Jones clusters containing up to 110 atoms. The Journal of Physical Chemistry A, 101(28), 5111-5116.

Wales, D. J., \& Scheraga, H. A. (1999). Global optimization of clusters, crystals, and biomolecules. Science, 285(5432), 1368-1372.

Xiong, Y., Huang, S., Chen, F., Ye, H., Wang, C., \& Zhu, C. (2012). The impacts of rapid urbanization on the thermal environment: A remote sensing study of Guangzhou, South China. Remote sensing, 4(7), 2033-2056. 


\section{CAPÍTULO 5. DISCUSIÓN Y CONCLUSIONES GENERALES}

En esta tesis doctoral, se presenta una metodología de armonización de datos mediante técnicas de cross-calibration a partir de zonas homogéneas, aplicable a las dos metodologías fundamentales para la calibración radiométrica de manera indirecta, como son los SNOs y las basadas en PICS. El objetivo principal de la misma, es la mejora de la interoperabilidad de los datos de sensores de satélite.

La metodología de SNOs fue desarrollada por NOAA-NESDIS hace más de una década para la calibración entre satélites meteorológicos de órbita polar y es actualmente una de las más utilizadas (Cao \& Heidinger 2002; Heidinger et al., 2002; Cao et al., 2004; Cao et al., 2005; Zou et al., 2006). Las SNOs minimizan las incertidumbres derivadas de las adquisiciones realizadas sobre la superficie de la Tierra por parte de los sensores de satélite, al observar el mismo target con apenas unos segundos de diferencia con idénticas condiciones de iluminación, geometría y atmósfera. Cao et al. (2004) establecieron como criterios para la determinación del área de SNO el cruce de órbitas entre satélites, una baja diferencia de tiempo entre adquisiciones, así como un límite máximo de distancia al nadir de $420 \mathrm{~km}$, quedando prácticamente restringidas las oportunidades de SNOs a las regiones polares de la Tierra. Estas restricciones limitaban bastante el proceso de cross-calibration a nivel espacial-temporal, por ello, se realizó una relajación y adaptación de algunos de los criterios establecidos por Cao et al. (2004) para la inter-comparación entre sensores realizándose principamente sobre los PICS (Helder et al., 2013; Angal et al., 2014; Mishra et al., 2014; Barsi et al., 2018). La crosscalibration entre sensores mediante la metodología de las SNOs sobre los PICS normalmente se realiza sin necesidad de que las orbitas entre satélites se crucen y sin que la diferencia entre adquisiciones simultaneas se produzcan en un periodo muy corto de tiempo (Lacherade et al., 2013; Helder et al., 2013; Chander et al., 2013; Barsi et al., 2108). Los PICS, al poseer una gran superficie homogénea utilizable para tareas de Cal/Val y una atmósfera relativamente estable, las restricciones utilizadas para la crosscalibration se relajan hasta el punto de que las observaciones entre sensores pueden realizarse incluso con días de diferencia (Tansock et al., 2015). Este método también contiene una limitación en los valores de reflectancia o radiancia obtenidos al estar restringidos a un determinado rango por las características de los PICS, la necesidad de modelización de la BRDF o la elevada latencia en la disponibilidad de datos sobre los PICS.

Para superar estas limitaciones de la cross-calibration sobre los PICS, se ha presentado y validado una metodología novedosa, global y ágil. El área de estudio de cada SNO está 
caracterizada y definida en base a criterios orbitales de cruce de las trayectorias terrestres entre satélites, al establecimiento de un umbral de 30 minutos de intervalo de tiempo entre adquisiciones y localización de áreas homogéneas donde realizar la comparativa entre sensores mediante un criterio de homogeneidad a partir del CV. Este método no necesita realizar ningún tipo de corrección atmosférica o de BRDF. Al ser una metodología global, los datos se adquieren sobre una gran variedad de superfices espectrales que permiten obtener un amplio rango de reflectancia para la comparativa entre sensores, además de permitir la localización de un gran número de oportunidades de SNOs. Esta metodología mejora notablemente los factores derivados de realizar la cross-calibration sobre los PICS.

A pesar de la diversidad de superficies utilizadas, el $\mathrm{R}^{2}$ muestra valores superiores a 0.997 en el caso de S2A y 0.999 en S2B, para todas las bandas a excepción de la banda NIR ancha (banda 8 de S2). La mayor incertidumbre se registra en los resultados de los pares de bandas con diferente RSRs. La validación con otros métodos confirma la robustez y los excelentes resultados obtenidos. Es importante resaltar los distintos resultados de S2A y S2B con diferencias entre ellos de aproximadamente un $1 \%$. Esta situación ha sido detectada por otros autores (Helder et al., 2018) y está actualmente bajo estudio por parte del MPC (ESA, 2020). El hecho de detectar esta problemática conocida, valida aún más el método de SNOs mediante zonas homogéneas.

Los resultados registrados en los pares de bandas con diferente RSRs, hicieron necesario realizar un análisis de esta situación. Aprovechando la ventaja que permitía la constelación S2 al contar cada satélite con 2 bandas en la misma región espectral, pero con RSRs muy diferentes, se adaptó la metodología de SNOs mediante zonas homogéneas hacia una metodología de SNOs ideales a partir de zonas homogéneas. Las adquisiciones de ambas bandas NIR de cada satélite S2 en un intervalo de tiempo de 0 segundos, eliminan las incertidumbres asociadas a efectos atmosféricos, de BRDF y de iluminación de la escena, permitiendo observar únicamente los efectos debidos a las diferencias en las RSRs entre bandas y a las superfices espectrales utilizadas. Esta situación también permite la posibilidad de automatizar la eliminación de outliers, ya que en las SNOs ideales, las nubes, sombras y cuerpos de agua son zonas válidas para la comparativa al ser la adquisición de forma simultánea en ambas bandas NIR. La comparativa de resultados de la metodología de SNOs Ideales con la metodología original de SNOs presenta una elevada coincidencia. Es importante resaltar la menor eliminación de outliers en la metodología de la SNOs ideal, de ahí las diferencias en la ponderación de algunas clases de usos de suelo con respecto a la metodología original de SNOs del capítulo 2. Estos resultados confirman la validez de la metodología de zonas homogéneas independientemente de la ponderación de usos de suelo utilizados o de que el intervalo de tiempo de la adquisición sea de 0 segundos o de 30 minutos, siempre y cuando los outliers sean eliminados. Se observa una menor incertidumbre en los resultados de los pares de bandas NIR de S2 A/B con una mayor diferencia en su RSRs 
adquiridos con el método de SNOs Ideales de 0 segundos, donde los valores de $\mathrm{R}^{2}$ son de 0.994 para S2A y de 0.992 para S2B, mientras que con la metodología de SNOs entre las bandas 8 de S2 A/B y la Banda 5 de L8 los valores de $R^{2}$ son de 0.980 y 0.984 respectivamente. Sería interesante contar en el futuro con sensores que posean bandas en la misma región del espectro electromagnético, pero con diferente RSRs para su estudio y análisis con la metodología de las SNOs ideales. Esto permitiría mejorar la interoperabilidad y armonización de series temporales de datos como por ejemplo el NDVI.

Actualmente, la metodología basada en el uso PICS presenta una serie de retos prioritarios, como son la armonización y actualización de los vigentes targets de referencia de calibración (ESA, 2017; Berthelot \& Henry, 2017). Según las recomendaciones realizadas por PICSCAR, es necesario establecer un acuerdo en la utilización de los AOI de calibración dentro de cada PICS (ESA, 2017; Berthelot \& Henry, 2017). En este trabajo, se han abordado estos retos con la aplicación del CV y de un algoritmo de optimización quasi-Newton combinado con la técnica de optimización global Basin-Hopping. Esta implementación ha permitido identificar una zona de interés mejorada, OGT, dentro de Libya-4. Este nuevo AOI posee unas características más homogéneas y estables, tanto temporal como espacialmente, con respecto la zona propuesta por CEOS, con diferencias entre el CV de ambas zonas del orden del $1 \%$ en las bandas VNIR de L8-OLI y S2-MSI. Además, esta metodología podría usarse en calibración vicaria para determinar la zona mas óptima de recogida de datos.

\subsection{Conclusiones}

En esta tesis doctoral, se presenta una metodología de armonización de datos de satélites mediante la utilización de zonas homogéneas. Esta metodología se ha implementado en las dos principales técnicas de cross-calibration, SNOs y PICS, para la mejora de las mismas. Los buenos resultados obtenidos sugieren que su utilización mejora la interoperabilidad de los datos generados. Esta metodología cobra especial relevancia en un contexto tanto actual como futuro, con una fuerte demanda de productos provenientes de satélites con sensores ópticos a bordo.

En estudios futuros sería interesante ampliar la metodología presentada, utilizando otras técnicas que permitan avanzar en la localización de zonas homogéneas (e.g. machine learning, big data, etc), mejorando así la robustez y solidez de la metodología presentada.

\subsection{Referencias}

Angal, A., Mishra, N., Xiong, X., \& Helder, D. (2014). Cross-calibration of Landsat 5 TM, and Landsat $8 \mathrm{OLI}$ with Aqua MODIS using PICS. In Earth Observing Systems XIX (Vol. 9218, p. 92180K). International Society for Optics and Photonics. 
Barsi, J. A., Alhammoud, B., Czapla-Myers, J., Gascon, F., Haque, M. O., Kaewmanee, M., ... \& Markham, B. L. (2018). Sentinel-2A MSI and Landsat-8 OLI radiometric cross comparison over desert sites. European Journal of Remote Sensing, 51(1), 822837.

Berthelot, B., \& Henry, P. (2017). Radiometric Calibration Workshop for European Missions.https://earth.esa.int/documents/700255/3194632/B\%2B\%C2\%AEatri ce+Berthelot PICSCAR-PPT-022-

MAGv1.0 BBerthelot PHenry 20170830.pdf/9ddad0e8-532c-4f6c-9f19404c8280755c (Accedido el 24 Mayo de 2020)

Cao, C., \& Heidinger, A. K. (2002). Intercomparison of the longwave infrared channels of MODIS and AVHRR/NOAA-16 using simultaneous nadir observations at orbit intersections. In Earth Observing Systems VII (Vol. 4814, pp. 306-316). International Society for Optics and Photonics.

Cao, C., Weinreb, M., \& Xu, H. (2004). Predicting simultaneous nadir overpasses among polar-orbiting meteorological satellites for the intersatellite calibration of radiometers. Journal of Atmospheric and Oceanic Technology, 21(4), 537-542.

Cao, C., Xu, H., Sullivan, J., McMillin, L., Ciren, P., \& Hou, Y. T. (2005). Intersatellite radiance biases for the High-Resolution Infrared Radiation Sounders (HIRS) on board NOAA-15,-16, and-17 from simultaneous nadir observations. Journal of Atmospheric and Oceanic Technology, 22(4), 381-395.

Chander, G., Hewison, T. J., Fox, N., Wu, X., Xiong, X., \& Blackwell, W. J. (2013). Overview of intercalibration of satellite instruments. IEEE Transactions on Geoscience and Remote Sensing, 51(3), 1056-1080.

ESA, (2017). Workshop on Radiometric Calibration for European Optical Missions, Frascati ESA/ESRIN, Italy. https://earth.esa.int/documents/700255/3194632/RadWS MoM Final.pdf/5fd 20f68-60b3-488a-a2bd-4732a3043a21 (Accedido el 24 de Mayo de 2020)

ESA, Sentinel-2 L1C Data Quality Report. https://sentinel.esa.int/documents/247904/685211/Sentinel-

2 L1C Data Quality Report (Accecido el 22 de Junio de 2020).

Heidinger, A. K., Cao, C., \& Sullivan, J. T. (2002). Using Moderate Resolution Imaging Spectroradiometer (MODIS) to calibrate advanced very high resolution radiometer reflectance channels. J. Geophys. Res, 107, 4702.

Helder, D., Markham, B., Morfitt, R., Storey, J., Barsi, J., Gascon, F., ... \& Lewis, A. (2018). Observations and Recommendations for the Calibration of Landsat $8 \mathrm{OLI}$ and Sentinel 2 MSI for improved data interoperability. Remote Sensing, 10(9), 1340. 
Helder, D., Thome, K. J., Mishra, N., Chander, G., Xiong, X., Angal, A., \& Choi, T. (2013). Absolute radiometric calibration of Landsat using a pseudo invariant calibration site. IEEE Transactions on Geoscience and Remote Sensing, 51(3), 1360-1369.

Lacherade, S., Fougnie, B., Henry, P., \& Gamet, P. (2013). Cross calibration over desert sites: Description, methodology, and operational implementation. IEEE Transactions on Geoscience and Remote Sensing, 51(3), 1098-1113.

Mishra, N., Haque, M. O., Leigh, L., Aaron, D., Helder, D., \& Markham, B. (2014). Radiometric cross calibration of Landsat 8 operational land imager (OLI) and Landsat 7 enhanced thematic mapper plus (ETM+). Remote sensing, 6(12), 12619-12638.

Tansock, J., Bancroft, D., Butler, J., Cao, C., Datla, R., Hansen, S., ... \& Murdock, T. (2015). Guidelines for radiometric calibration of electro-optical instruments for remote sensing.

Zou, C. Z., Goldberg, M. D., Cheng, Z., Grody, N. C., Sullivan, J. T., Cao, C., \& Tarpley, D. (2006). Recalibration of microwave sounding unit for climate studies using simultaneous nadir overpasses.Journal of Geophysical Research: Atmospheres, 111(D19). 


\section{CAPÍTULO 6. LISTA DE PUBLICACIONES}

Para la presente tesis doctoral, han sido publicados dos trabajos de investigación relacionadas con la misma, en Remote Sensing Journal (ISSN: 2072-4292) Special Issue "Cross-calibration and Interoperability of Remote Sensing Instruments" con un índice de impacto de 4.118 y 7/30 (Q1) en el año 2019 y de 4.509 y 9/30 (Q2) en el año 2020 en 'Remote Sensing' en la clasificación de la categoría JCR.

Revista: Remote Sensing Journal, Special Issue "Cross-calibration and Interoperability of Remote Sensing Instruments". Impact Factor (JCR, 2019) = 4.118

Artículo: "Analysis of Spatial and Temporal Variability in Libya-4 with Landsat 8 and Sentinel-2 Data for Optimized Ground Target Location"

Día de publicación: 05/12/2019

DOI: Remote Sens. 2019, 11(24), 2909;

https://doi.org/10.3390/rs11242909

Citar: Rodrigo, J.F.; Gil, J.; Salvador, P.; Gómez, D.; Sanz, J.; Casanova, J.L. Analysis of Spatial and Temporal Variability in Libya-4 with Landsat 8 and Sentinel-2 Data for Optimized Ground Target Location. Remote Sens. 2019,11, 2909. https://doi.org/10.3390/rs11242909

Revista: Remote Sensing Journal, Special Issue "Cross-calibration and Interoperability of Remote Sensing Instruments". Impact Factor (JCR, 2020) = 4.509

Artículo: "An Empirical Radiometric Intercomparison Methodology Based on Global Simultaneous Nadir Overpasses Applied to Landsat 8 and Sentinel-2"

Día de publicación: 24/08/2020

DOI: Remote Sens. 2020, 12(17), 2736;

https://doi.org/10.3390/rs12172736

Citar: Gil, J.; Rodrigo, J.F.; Salvador, P.; Gómez, D.; Sanz, J.; Casanova, J.L. An Empirical Radiometric Intercomparison Methodology Based on Global 
Simultaneous Nadir Overpasses Applied to Landsat 8 and Sentinel-2. Remote Sens. 2020, 12, 2736. https://doi.org/10.3390/rs12172736 